\title{
Measurements of \\ Radioactivity
}

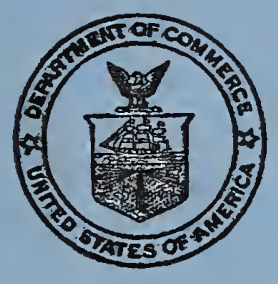

United States Department of Commerce

National Bureau of Standards

Circular 476 

UNITED STATES DEPARTMENT OF COMMERCE - Charles Sawyer, Secretary NATIONAL BUREAU OF STANDARDS - E. U. Condon, Director

\section{Measurements of \\ Radioactivity}

by Leon F. Curtiss

National Bureau of Standards Circular 476 Issued October 15, 1949

For sale by the Superintendent of Documents, U. S. Government Printing Office, Washington 25, D. C. Price $\$ 20$ 


\section{P r e f a ce}

This publication is a compilation of information intended to be useful to those starting out in research on problems in radioactivity and nuclear physics. An effort has been made to provide, in brief form, most of the essential background for all phases of experimental investigations in this general field. The number of topics involved limits the space that could be given to each, and the author is aware that for many items this has resulted in the elimination of important information. This defect may be corrected in future revisions, when criticisms and suggestions have indicated the direction of most useful expansion. This Circular is issued in its present form to provide a convenient source of the information contained and to provide an opportunity for those interested in this field to offer critical comments that can serve as the basis of an improved compilation.

E. U. Condon, Director. 
Preface

I. Introduction

1. Discovery

2. Statistical nature

3. Radioactive radiations

4. Artificial radioactivity

II. Detection and measurement of radioactive radiations

1. Photographic emulsions

2. Crystals

3. Ionization of gases.

4. Measurement of charge transported by alpha and beta rays.

5. Counting methods

6. Ionization chambers

7. Proportional counters

8. Geiger-Müller counters

9. Electron multiplier

10. Wilson cloud chamber.

11. Calorimetric methods of measuring radioactive radiations ..........................

III. Measurement of ionization currents.

1. Electroscopes

2. Quartz fiber electroscopes

3. Electrometers

4. String electrometers

5. Portable electrometers

6. Electrometer tubes

7. Vibrating reed electrometers.

8. Ionization chambers

IV. Radioactive substances

1. Natural radioactivity

2. Artificial radioactivity

3. Disintegration by protons

4. Disintegration by deuterons.

5. Disintegration by alpha particles

6. Distintegration by neutrons.

7. Disintegration by photons.

8. Alternate methods for producing a given radioisotope

9. Decay of artificially radioactive sub-

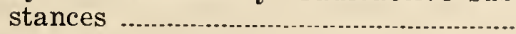

10. Positron emission

11. Electron captura

12. Isomeric transitions

V. Equipment for producing artificially radioactive isotopes

1. Voltage multiplier

2. Cascade transformers

3. Van de Graaf generator.

4. Linear accelerator

5. Cyclotron

6. Betatron

7. Synchrotron

8. Uranium reactors

9. Fission products
VI. Radioactive radiations

1. Neutrons

2. Production of neutrons.

3. Mass of the neutron.

4. Measurements of neutrons.

5. Fast neutrons

6. Slow neutrons

7. Counting alpha particles produced by neutron-induced distintegration ........

8. Counting gamma rays resulting from neutron capture

9. Induced activities

10. Cross sections for reactions with neutrons

11. Radioactivity produced by neutrons....

12. Proton

13. Production of protons

14. Detection and measurement of protons

15. Radioactivity produced by proton bombardment

16. Deuterons

17. Deuteron reactions

18. Alpha particles

19. Range of alpha particles.

20. Bragg curve

21. Stopping power

22. Geiger-Nuttall law

23. Scattering of alpha particles.................

24. Emission of alpha particles from the nucleus

25. Beta particles

26. Measurement of beta rays.

27. Measurements of beta rays with end window counters

28. Beta-ray standards

29. Electroscopes

30. Ionization chambers

31. Beta-ray spectra

32. Magnets for semicircular beta-ray spectrographs

33. Magnetic electron lens spectrometer......

34. Continuous beta-ray spectra.

35. Neutrino

36. Fermi theory of beta-ray decay.

37. Absorption of beta particles.

38. Scattering of beta particles.

39. Range of beta particles.

40. Absorption method for determining energies of beta rays............................

41. Beta-ray energies by magnetic deflection

42. Positron

43. Annihilation radiation and pair production

44. Mesotrons

45. Artificial production of mesotrons.........

46. Gamma rays

47. Disintegration schemes .

48. Coincidence measurements

49. Absolute calibration of gamma-ray sources by coincidence. 
50. Measurement of gamma radiation......... 64

51. Counting gamma rays............................ 65

52. Fission particles ...................................... 65

53. Measurement of half-value periods....... 67

54. Counter measurement of short halfvalue periods

55. Measurement of half-value periods with counters

56. Determination of very long half-periods

57. Half-periods from true coincidence measurements

58. Statistical error in measurement of half-value periods with counters...

VII. Radioactive tracers

1. Preparation and measurement of tracer samples

2. Absolute measurements of tracer samples

VIII. Radioactive standards and units

1. Alpha-ray standards

2. Beta-ray standards

3. Gamma-ray standards
4. Neutron standards

5. Units used in radioactive measurements

IX. Radioactivity in geology

1. Uranium lead ratio.

2. Helium content

3. Age of terrestial matter.

4. Determination of radium and thorium in rocks

5. Pleochroic halves

6 . Generation of heat in the earth.

X. Health protection

1. Nature of radiation injuries

2. Genetic effects of radiation

3. Visible evidence of injury from radiation

4. Tolerance limits

5. Measurements of tolerance levels 82

6. Alpha radiation

7. Protective precautions for substances emitting alpha rays

8. Protection from gamma rays

XI. References 


\section{MEASUREMENTS OF RADIOACTIVITY}

\section{INTRODUCTION}

\section{Discovery}

The discovery of radioactivity illustrates how an incorrect hypothesis may stimulate experiments that lead to the discovery of new phenomena. After Roentgen had announced the discovery of X-rays in 1895, a search was instituted by other physicists for substances that might emit naturally a similar radiation. Becquerel concluded that since X-rays stimulated certain substances to a strong phosphorescence, such substances would be likely to emit a radiation having the properties of X-radiation. Accordingly, he exposed photographic plates, wrapped in black paper, to a number of phosphorescent substances with uniformly negative results until he tried salts of uranium. The success of this trial, announced in 1896, marks the discovery of radioactivity.

\section{Statistical Nature}

The fundamental nature of radioactivity can be described as a probability that the atoms of a substance will undergo a transmutation spontaneously into atoms of another element with a release of energy in the form of various types of radiation. If this probability is very small the substance is either stable or weakly radioactive. If it is large it is strongly radioactive. To test this view, let us assume that the probability that an atom of a given substance will disintegrate in an interval of time $\Delta$ is $p$, which is the same for all atoms of the substance and independent of their age. For very small values of $\Delta$ the probability, $p$, will be proportional to the length of the interval so that $p=\lambda \triangle$, where $\lambda$ is a constant representing the probability that an atom will disintegrate in unit time. Now the probability, $q$, that an atom will not disintegrate in an interval $\triangle$ is $q=1-\mathrm{p}=1-\lambda \triangle$. For a finite time $t=\dot{k} \triangle$, the probability that an atom will not be transformed is $q^{\prime}=(1-\lambda \triangle)^{k}=$ $(1-\lambda \triangle)^{t / \Delta}$, which may be written

$$
q^{\prime}=\left[(1-\lambda \triangle)^{-1 / \lambda \Delta}\right]^{-\lambda t} .
$$

Now let $\triangle$ become infinitely small while keeping the product $k \Delta$ constant, that is $t$ constant,

$$
q^{\prime}=\lim _{\Delta \rightarrow 0}\left[(1-\lambda \triangle)^{-1 / \lambda \Delta}\right]^{-\lambda t} .
$$

We note that

$$
(1-\lambda \triangle)^{-1 / \lambda \Delta} \text { has the form }(1+x)^{1 / x} \text {, }
$$

the limit of which, as $x$ approaches zero, is $e$. Therefore

$$
q^{\prime}=e^{-\lambda t} .
$$

If at the time $t=0$ there is a large number $N_{0}$ of radioactive atoms present, the number $N$, at any subsequent time $t$, is given by

$$
N=N_{0} e^{-\lambda t} .
$$

This relation expresses the law of exponential decay so widely established experimentally in the study of radioactivity, where $\lambda$ is the disintegration constant.

Differentiating eq. 2 with respect to $t$, we obtain for the rate of disintegration

$$
\frac{d N}{d t}=\lambda N_{0} e^{-\lambda t}=-\lambda \mathrm{N},
$$

the negative sign indicating that the number of unchanged atoms is decreasing.

It has become customary to identify the rates of disintegrations of radioactivity atoms by specifying the time required to decay to onehalf the initial activity. This time is known as the half-value period, $T$. This period is the time, $T$, required for $N / N_{0}=1 / 2=e^{-\lambda T}$; or $\log _{\mathrm{e}} 2=$ $-\lambda T$, from which $T=0.693 / \lambda$.

Another constant that may be derived from the fundamental equation is the mean life, $L$, of the whole group of atoms. The number of atoms transformed in time $d t$ is given by $\lambda N d t=$ $\lambda N_{0} e^{-\lambda t} d t$. Each atom has a life $t$, hence the average life of all of them is

$$
L={ }_{0} \int \infty \frac{t \lambda N_{0} e^{-\lambda t} d t}{N_{0}}={ }_{0} \int \infty t \lambda e^{-} \lambda^{t} d t=1 / \lambda .
$$

(a). Successive transformations.-It is of interest to determine the relative amounts of radioactive substances when atoms of one type disintegrate successively into other types. These can be determined from the relation $N=N_{0} e^{-\lambda t}$ for a number of conditions that are important in practice. To derive these relations we will assume that we have a radioactive chain consisting of three types of atoms, $A, B$, and $C$, in which $A$ disintegrates to form $B$, which in turr disintegrates into $C$ with the respective disintegration constants $\lambda_{1}, \lambda_{2}$, and $\lambda_{3}$. Let the respective number of atoms at time $t$ be $P, Q$, and $R$, and $P_{0}, Q_{0}$, and $R_{0}$ at time $t=0$. The relations 
can be extended to a chain of any number of members, but for simplicity three will be considered here. The mathematical analysis was developed by Rutherford [1] ${ }^{1}$ and is given here for a few typical cases.

Consider first that all atoms are initially of type $A$. Hence at $t=0, P=P_{0}, Q=0$, and $R=0$. From the fundamental expression for radioactive decay, at any time $t$

$$
P=P_{0} e^{-\lambda_{1} t}
$$

The rate of change of atoms of $A$ into those of $B$ is $d P / d t=-\lambda_{1} P$, and the rate of increase of atoms of $B$ is this rate decreased by that at which atoms of $B$ change into those of $C$, which is $+\lambda_{2} Q$.

Therefore

$$
\frac{d Q}{d t}=\lambda_{1} P \cdot-\lambda_{2} Q
$$

and similarly,

$$
\frac{d R}{d t}=\lambda_{2} Q-\lambda_{3} R
$$

Now

$$
\frac{d Q}{d t}=\lambda_{1} P_{o} e^{-\lambda_{1}{ }^{\prime}-\lambda_{2} Q},
$$

from which

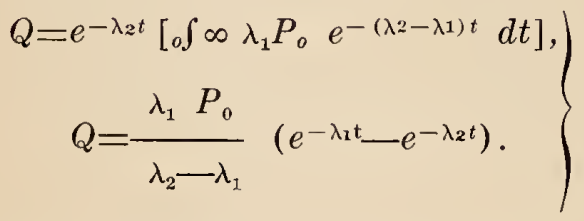

The value of $Q$ has a maximum when

$$
t=\frac{\ln \lambda_{1}-\ln \lambda_{2}}{\lambda_{1}-\lambda_{2}}
$$

Substituting the value of $Q$ in the expression for $d R / d t$ and solving for $R, R=P_{0}$

$$
\begin{gathered}
{\left[\frac{\lambda_{1} \lambda_{2}}{\left(\lambda_{2}-\lambda_{1}\right)\left(\lambda_{3}-\lambda_{1}\right)} e^{-\lambda_{1} t}+\frac{\lambda_{1} \lambda_{2}}{\left(\lambda_{1}-\lambda_{2}\right)\left(\lambda_{3}-\lambda_{2}\right)} e^{-\lambda_{2} t}\right.} \\
\left.+\frac{\lambda_{1} \lambda_{2}}{\left(\lambda_{1}-\lambda_{3}\right)\left(\lambda_{2}-\lambda_{3}\right)} e^{-\lambda_{3}}\right] \cdot \quad \text { (6) }
\end{gathered}
$$

A second case is that in which atoms of $A$ have been produced at a constant rate and the process continued until the atoms of $A, B$, and $C$ have reached a steady limiting value. Then the production of $A$ is stopped abruptly. Let $n_{0}$ represent the number of atoms of $A$ produced per unit time. Then at equilibrium

$$
n_{0}=\lambda_{1} P_{E}=\lambda_{2} Q_{E}=\lambda_{3} R_{E}
$$

where the subscript $E$ indicates the steady state value. At the moment when the production of $A$ is stopped, $t=0$.

Then

$$
\begin{aligned}
& P=P_{E}=n_{0} / \lambda_{1}, \\
& Q=Q_{E}=n_{0} / \lambda_{2}, \\
& R=R_{E}=n_{0} / \lambda_{3},
\end{aligned}
$$

Therefore, from the fundamental relation $N=N_{0} e^{-\lambda t}$ and the initial conditions,

$$
\begin{gathered}
P=\frac{n_{0}}{\lambda_{1}} e^{-\lambda_{1} t}, \\
Q=\frac{n_{0}}{\lambda_{1}-\lambda_{2}}\left(\frac{\lambda_{1}}{\lambda_{2}} e^{-\lambda_{2}}-e-{ }^{\lambda 1 t}\right),
\end{gathered}
$$

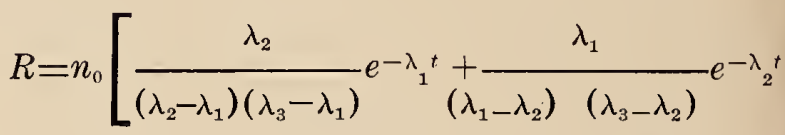

$$
\begin{aligned}
& \left.+\frac{\lambda_{1} \lambda_{2}}{\lambda_{3}\left(\lambda_{1}-\lambda_{3}\right)\left(\lambda_{2}-\lambda_{3}\right)} e^{-\lambda_{3} t}\right] \text {. }
\end{aligned}
$$

A third case, a modification of the preceding example, is the condition that atoms of $A$ have been produced at a constant rate for a time $T$ and then the process interrupted. If $n_{0}$ is the rate of production of atoms of $A$ it is required to find the number of atoms of $A, B$, and $C$ at any time subsequent to $T$.

The number of atoms of $A$ at time $T, A=P_{T}$ is given by $P_{T}=n_{0} \int_{0} \int^{T} e^{-\lambda_{1} t} d t=\frac{n_{0}}{\lambda_{1}}\left(1-e^{-\lambda_{1} T}\right)$. At any time $t$ subsequent to $T$,

$$
P=P_{T} e^{-\lambda_{1} t}=\frac{n_{0}}{\lambda_{1}}\left(1-e^{-\lambda_{1}{ }^{T}}\right) e^{-\lambda_{1} t} .
$$

To determine $Q_{T}$, we note that in time $d t$ there are $n_{0} d t$ atoms of $A$ produced. Therefore the number of atoms $d Q$ of $B$ resulting from the change in $A$ is obtained from eq. 5 as 


$$
d Q=\frac{n_{0} \lambda_{1}}{\lambda_{1}-\lambda_{2}}\left(e^{-\lambda_{2} t}-e^{-\lambda_{1} t}\right) d t=n_{0} f(t) d t .
$$

After a time of exposure $T$, the number of particles $Q_{T}$ of type $B$ present is given by

$$
\begin{gathered}
Q_{T}=n_{0}[f(T) d t+f(T-d t) d t+\ldots+f(0) d t] \\
=n_{0} f^{T} f(t) d t, \\
=\frac{n_{0} \lambda_{1}}{\lambda_{1}-\lambda_{2}}{ }_{0}^{T}(12 \mathrm{a}) \\
=\frac{n_{0} \lambda_{1}}{\lambda_{1}-\lambda_{2}}\left[\frac{1-e^{-\lambda_{2} T}}{\lambda_{2}}-\frac{1-e^{-\lambda_{1} T}}{n_{1}}\right] .(12 \mathrm{~d})
\end{gathered}
$$

In the same way the number $Q$ at any time $t$ subsequent to $T$ is given by

$$
\begin{gathered}
Q=n_{0 t} f^{T+t} f(t) d t \\
=\frac{n_{0} \lambda_{1}}{\lambda_{1}-\lambda_{2}}\left[\frac{1-e^{-\lambda_{2} T}}{\lambda_{2}} e^{-\lambda_{2} t}-\frac{1-e^{-\lambda_{1} T}}{\lambda_{1}} e-\lambda_{1}{ }^{t}\right]
\end{gathered}
$$

From $12 d$ and $13 b$ we get

$$
\frac{Q}{Q_{T}}=\frac{\frac{1-e^{-\lambda_{2} T}}{\lambda_{2}} e^{-\lambda_{2} t}-\frac{1-e^{-\lambda_{1}{ }^{T}}}{\lambda_{1}} e^{-\lambda_{1}{ }^{t}}}{\frac{1-e^{-\lambda_{2} T}}{\lambda_{2}}-\frac{1-e^{-\lambda_{1}{ }^{T}}}{\lambda_{1}}} .
$$
out

The corresponding ratio for atoms of $R$ comes

$$
\frac{R}{R_{t}}=\frac{a e^{-\lambda_{1} t}+b e^{-\lambda_{2} t}+c e^{-\lambda_{3} t},}{\mathrm{a}+\mathrm{b}+\mathrm{c}}
$$

where

$$
\begin{aligned}
& \mathrm{a}=\frac{\lambda_{2}}{\left(\lambda_{2}-\lambda_{1}\right)\left(\lambda_{3}-\lambda_{1}\right)}\left(1-e^{-\lambda_{1} T}\right), \\
& \mathrm{b}=\frac{\lambda_{1}}{\left(\lambda_{1}-\lambda_{2}\right)\left(\lambda_{3}-\lambda_{2}\right)}\left(1-e^{-\lambda_{2}{ }^{T}}\right), \\
& c=\frac{\lambda_{1} \lambda_{2}}{\left(\lambda_{1}-\lambda_{3}\right)\left(\lambda_{2}-\lambda_{3}\right)}\left(1-e^{-\lambda_{3}{ }^{T}}\right),
\end{aligned}
$$

A final example is represented by the growth of the atoms of $A, B$, and $C$ from zero activity where $A$ is produced at a constant rate and it is desired to know the numbers of the various atoms at some time $t$. These values can be deduced from the second case where production of active atoms has reached a limiting value, in which the numbers of atoms are represented by $P_{E}, Q_{E}$, and $R_{E}$. Equilibrium having been established, assume that all radioactive atoms are removed and a new supply continues to build up at the same constant rate. For the atoms that have been removed we have as before,

$$
\begin{gathered}
P=P_{E} e^{-\lambda_{1} t}, \\
Q=\frac{Q_{E}}{\lambda_{1}-\lambda_{2}}\left(\lambda_{1} / \lambda_{2} e^{-\lambda_{2} t}-e^{-\lambda_{1} t}\right), \ldots
\end{gathered}
$$

In the new group being formed there will be amounts present represented by $P_{1}, Q_{1}$, and $R_{1}$. However, it is obvious that the removal of the equilibrium group does not change the total number of each kind of atoms present, since this would imply that the disintegration rate or the rate of production would depend on the presence of the parent atom, which is not the case. Therefore:

$$
\begin{aligned}
& P_{1}+P=P_{\mathrm{E}} \quad \text { or } \quad P_{1}=P_{E}-P \\
& Q_{1}+Q=Q_{E} \quad Q_{1}=Q_{E}-Q \\
& R_{1}+R=R_{E} \quad R_{1}=R_{E}-R
\end{aligned}
$$

Substituting in eq. 7,8 , and 9 we have

$$
\begin{gathered}
P_{1}=P_{E}\left(1-e^{-\lambda_{1} t}\right), \\
Q_{1}=Q_{E}\left[1-\frac{\left(\lambda_{1} e^{-\lambda_{2} t}-\lambda_{2} e^{-\lambda_{1} t}\right)}{\lambda_{1}-\lambda_{2}}\right] .
\end{gathered}
$$

$R_{1}=R_{E}\left[1-\lambda_{3}\right.$

$$
\left.\begin{array}{c}
\left(\frac{\lambda_{2}}{\left(\lambda_{2}-\lambda_{1}\right)\left(\lambda_{3}-\lambda_{1}\right)} e^{-\lambda_{1} t}+\frac{\lambda_{1}}{\left(\lambda_{1}-\lambda_{2}\right)\left(\lambda_{3}-\lambda_{2}\right)}\right. \\
e^{-\lambda_{2} t}+\frac{\lambda_{1} \lambda_{2}}{\lambda_{3}\left(\lambda_{1}-\lambda_{3}\right)\left(\lambda_{2}-\lambda_{3}\right)} e^{-\lambda_{3} t}
\end{array}\right] .
$$

We note that $P_{1}, Q_{1}$, and $R_{1}$ represent the growth of activity from zero and therefore are the values required for this situation.

On comparison it will be found that the curves showing the growth of activity from zero and those showing the decay for the same initial and final activities are complementary. For the 
simple case of atoms of one kind this is illustrated in figure 1, which shows the curve for $e^{-\lambda t}$ and $\left(1-e^{-\lambda t}\right)$ in relation to the half period

$T$ for any radioactive element.

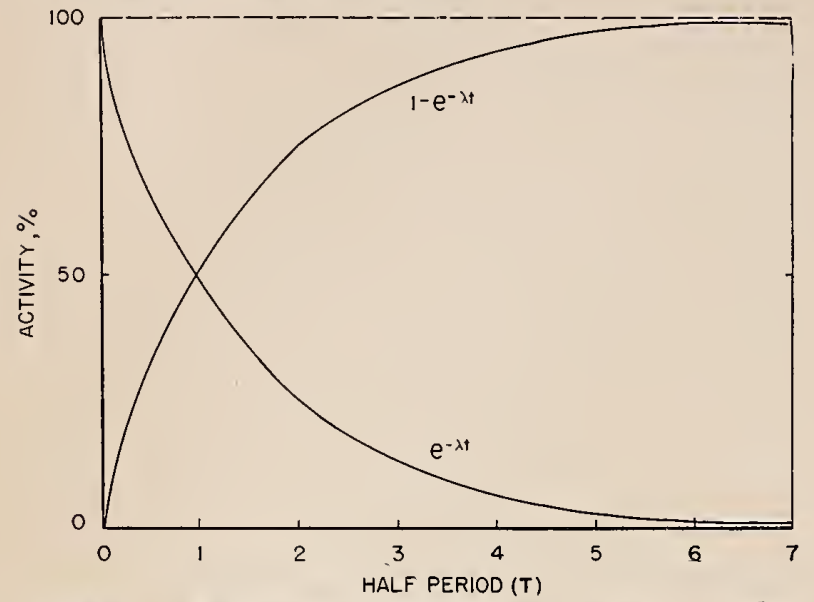

FIGURE 1. Exponential growth and decay of radioactive atoms.

In general, since the parent atoms of any radioactive chain are undergoing disintegration, it is never strictly possible to establish complete equilibrium. Wherever the disintegration constant of the parent is very small compared with those of succeeding members, equilibrium will be practically complete. This situation is described as secular equilibrium. In those cases where the disintregration constants are more nearly comparable, a transient equilibrium is established.

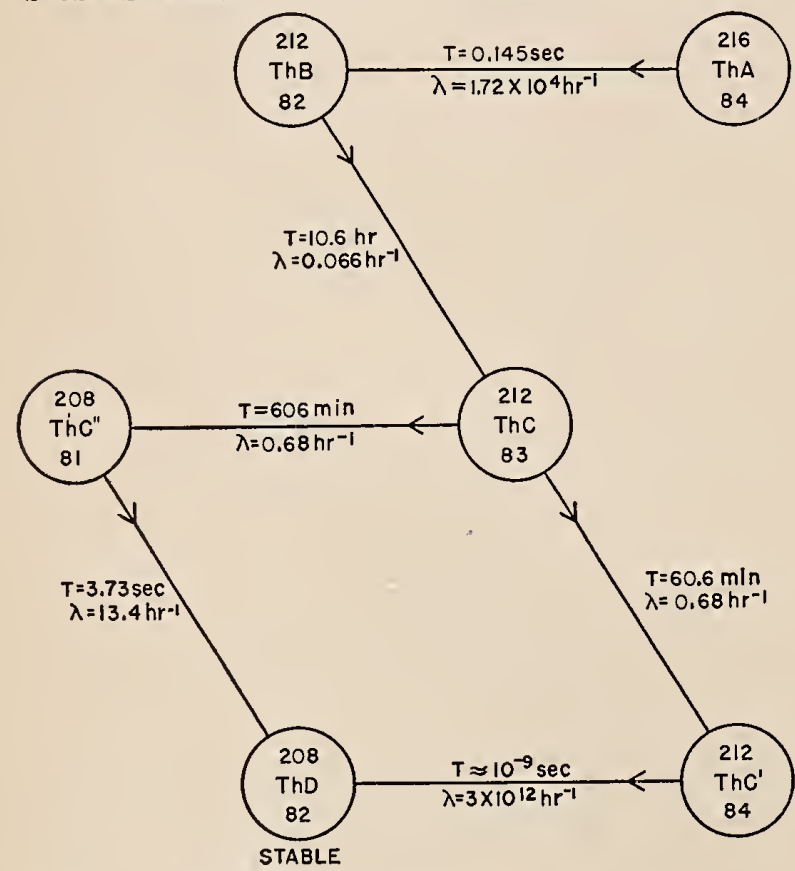

FIGURE 2. Radioactive products of thoron.

An interesting case of transient equilibrium is provided by the active deposit from thoron.
The disintegration product of this gas is a solid, which can be collected on a negatively charged electrode in the gas. The products form a chain, represented by figure 2 . It will be noted that because of its short half-period, ThA will completely disappear shortly after the electrode is removed from the thoron, and that of the remaining members of the chain only $T \mathrm{hC}$ and $T h C^{\prime}$ emit alpha particles. The half-period of $T h C^{\prime}$ is extremely short, so that it will be in practically complete equilibrium with the $T h C$. Therefore, we need only consider the growth curves of the $T \mathrm{~h} B$ and $T \mathrm{~h} C$ if measurements of alpha ray activity of the deposit are to be made. Since $T \mathrm{~h} B$ does not emit alpha rays, observations of alpha rays will show only the radiations from $T h C$ and $T h C^{\prime}$. At the time of removal after a short exposure, practically all atoms will be those of $\operatorname{Th} B$, so that no alpha activity will be observable initially. The relative growth and decay of the alpha ray activity will be represented by the quantity $\left(e^{-\lambda_{1} t}-e^{-\lambda_{2} t}\right)$, where $\lambda_{1}$ is the disintegration constant of $T \mathrm{~h} B$ $=0.0656 \mathrm{hr}^{-1}$, and $\lambda_{2}$ is the constant for $T \mathrm{~h} C=$

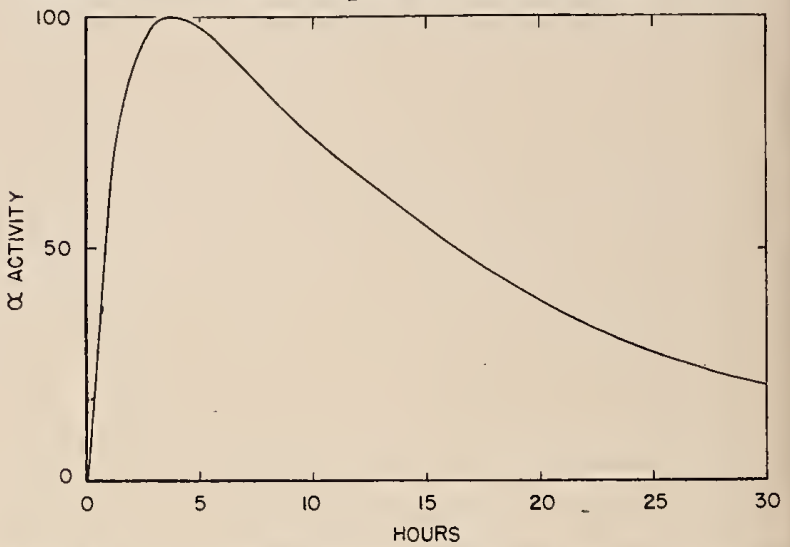

FIgURE 3. Curve showing growth and decay of alpharay activity on the radioactive deposit obtained from thoron, as measured by alpha particles.

$0.684 \mathrm{hr}^{-1}$. Plotting the results in terms of the maximum activity as 100 , the graph shown in figure 3 is obtained. After $5 \mathrm{hr}$ the activity decays practically with the half-period of $T \mathrm{~h} B$, showing that the $T \mathrm{TC}$ and the $T \mathrm{~T} C^{\prime}$ (already in secular equilibrium with $T h C$ ), which emit the alpha particles observed, are in secular equilibrium with the $T h B$.

(b) Fluctuations in activity.-The statistical conception of the process of radioactive disintegration, in which $\lambda$ is regarded as a probability, leads at once to the expectation of fluctuations in the observed numbers of disintegration per unit time. These fluctuations become more prominent the smaller the number of atoms under observation. Since $\lambda d t$ is the probability for a radioactive atom disintegrating in time $d t$, then $p=1-e^{-\lambda t}$ is this probability for a time, $t$. The corresponding probability that an atom does not disintegrate is $q=e^{-\lambda t}$. From the bi- 
nominal law of probabilities the probability of an event occurring $r$ times in $N$ independent trials is

$$
P_{r}=p^{r} \frac{N !}{r !(N-r) !} q^{N-r}
$$

which can be written in the approximate form, known as the Poisson formula

$$
P_{r}=\frac{(N p)^{r}}{r !} e^{-N p}
$$

which gives a good approximation when $r$ is small compared with $N$, and $p$ represents the probability of a rare event in a single trial. Since $N p$ is the expected number of successes, it can be put equal to $\epsilon$ giving

$$
P_{r}=\frac{\epsilon}{r !} e^{-\epsilon}
$$

For example, assume a large number $N$ of radioactive atoms, which on the average disintegrate at the rate of 10 per minute. Then $N p=\varepsilon=10$. The Poisson formula gives the values of $p_{r}$ for various values of $r$ disintegrations per minute shown in table 1 . Since the Poisson formula gives a good approximation only when $r$ is small compared with $N$, in its application to radioactive disintegration where $p=\lambda$ and $\epsilon=N \lambda$, this requires $\lambda$ to be small compared with $N$. This requirement can also be stated as demanding that the interval for which $r$ is taken be small compared with $1 / \lambda$, the mean life of the atom.

\section{TABLE 1.}

\begin{tabular}{|l|l|l|l|}
\hline$r$ & \multicolumn{1}{|c|}{$P_{r}$} & \multicolumn{1}{c|}{$P_{r}$} \\
\hline $0 \ldots \ldots \ldots \ldots$ & $4.6 \times 10^{-5}$ & 8 & \multicolumn{1}{c|}{$P$} \\
\hline$\ldots \ldots \ldots \ldots$ & $4.6 \times 10^{-4}$ & 10 & .125 \\
$2 \ldots \ldots \ldots \ldots$ & $2.3 \times 10^{-3}$ & 12 & .095 \\
$4 \ldots \ldots \ldots \ldots$ & 0.019 & 40 & $5.6 \times 10^{-14}$ \\
\hline
\end{tabular}

From the theory of probability the standard deviation $E$, in a large number of observations, $N$, of two events with probabilities $p$ and $q$ is

$$
E= \pm \sqrt{N p q}
$$

In the case of radioactive transformations $p=\lambda \tau$ is the probability that an atom disintegrates in time, $\tau$, short compared with the halfperiod of the atoms, and $q=1-\lambda \tau$ is the probability that an atom does not disintegrate in this time. Therefore:

$$
E= \pm \sqrt{N \lambda \tau(1-\lambda \tau)}= \pm \sqrt{N\left(\lambda \tau-\lambda^{2} \tau^{2}\right)}
$$

neglecting $\lambda^{2} \tau^{2}$ in comparison with $\lambda \tau$

$$
E= \pm \sqrt{N \lambda \tau}= \pm \sqrt{Z}
$$

where $Z$ is the average number of atoms disintegrating in time $\tau$. Therefore the actual number shows fluctuations of the order of $\pm \sqrt{Z}$ so that the absolute error increases with $Z$, but the relative error $\sqrt{Z / Z}$ decreases. This illustrates the well-known increase of accuracy of observations with increase of total number of disintegrations observed.

\section{Radioactive Radiations}

The radiations emitted as the result of radioactive transformations or which may produce radioactivity in atoms when absorbed by them, known at present are:

a. Alpha (a) rays, or particles, that are the nuclei of helium atoms. These are ejected from some radioactive nuclei, usually with considerable kinetic energy, and result in a new nucleus with a nuclear charge lower by two elementary units than the parent nucleus.

b. Beta $\left(\beta^{-}\right)$rays, which are negative electrons emitted by the nucleus and thus result in a nucleus with an increase of one elementary unit of charge.

c. Positrons $\left(\beta^{+}\right)$, which are positive electrons ejected from the nucleus producing a decrease of one unit in the charge of the nucleus.

d. Gamma $(\gamma)$ rays, an electromagnetic radiation from the nucleus that produces no change in nuclear charge but accompanies transmutation of the nucleus.

e. Conversion electrons $\left(e^{-}\right)$, which are orbital electrons ejected by photoelectric conversion of nuclear gamma rays in the orbital levels of the same atom. Since they do not arise from the nucleus, they do not result in a transmutation.

f. X-rays emitted when orbital electrons ejected from inner shells or captured by the nucleus, are replaced by electrons from outer levels.

g. Also occurring but possibly falling into separate categories are:

1. Neutrino, which, according to present theories of beta-disintegration, is emitted with the negative or positive electron in nuclear transformations, and theoretically accounts for the continuous beta spectrum and differences in spin quantum numbers. Thus far there is no really conclusive experimental evidence showing the existence of the neutrino.

2. Neutron, which, although it is the product of a nuclear transmutation, is emitted simultaneously with formation of the compound nucleus, with the possible exception of fission neutrons. The neutron is itself unstable. 
3. Meson, which occurs only in nuclear processes involving tremendously high energies, as in cosmic ray phenomena. These have also been produced artificially in the University of California cyclotron, and are also unstable.

h. The following particles are of importance in radioactivity since when they are captured by a stable nucleus they may render it radioactive and are also emitted during the process of forming the new nucleus, although they are not associated with the subsequent radioactive disintegration of the newly formed nuclei.

1. Neutron $(n)$ of unit atomic mass and no charge.

2. Proton $(p)$ with two units of mass and one positive charge.

3. Deuteron (d) with two units of mass and one positive charge.

4. Triton $(t)$ with three units of mass and one positive charge.

5. Alpha particle (a) with four units of mass and two of positive charge.

6. Electron $(e)$ with zero units of mass and one of negative charge.

7. Gamma ray $(\gamma)$ with zero units of mass and no charge.

\section{Artificial Radioactivity}

Although radioactivity was originally discovered and investigated in connection with elements existing in nature that were undergoing transmutations, it has now become recognized as a general property of atomic nuclei. Joliot and Curie discovered that stable substances may be made radioactive in 1934, while studying the effect of bombardment of nuclei of low atomic number by alpha particles. Since that time it has been found possible to produce radioactive isotopes of practically every atomic species. Furthermore, these artificially induced radioactivities conform to the general laws established for radioactivity previously known to occur in nature. Since radioactivity is a property of the nucleus it must be explained in terms of hypotheses and experiments that involve the structure of the nucleus.

(a) Structure of the atomic nucleus.-Ideas regarding the components of the inucleus and their interaction have undergone many modifications since the initial discovery of radioactivity. Some of the difficulties in interpreting radioactive processes in terms of nuclear structure have been removed by the present hypothesis on this subject. Since the discovery of the neutron in 1932 by Chadwick, the nucleus has come to be regarded as made up of protons and neutrons. The number of protons determines the nuclear charge $Z$, and therefore the chemical behavior of the nucleus and its position in the periodic table. The sum of the number of protons and neutrons determines the atomic mass number of the nucleus, $A$.

Any combination of protons and neutrons which, when associated in a nucleus, has a low probability of disintegration into some other combination is stable. Ultimately all unstable forms tend to transform until a stable arrangement is reached, and therefore are radioactive. Instability appears in the very simplest of the structural elements of the nucleus. The neutron itself, when produced in the free state, quickly transforms, presumably into a proton. The mechanism of this transformation has not been definitely established but it may be assumed, in absence of evidence to the contrary, that the neutron emits a beta particle that converts it into a proton. The proton in turn quickly picks up an orbital electron to form hydrogen, the first stable chemical element in the periodic table. We know now that hydrogen can exist in three forms. Adding one neutron to the nucleus of a hydrogen atom produces a deuteron, a nucleus with two atomic mass units and one nuclear charge. Chemically indistinguishable from the more common variety of hydrogen, this element is stable. However, when a second neutron is added to the hydrogen nucleus the combination becomes unstable. A radioactive element results that behaves chemically as hydrogen but can be distinguished from the common variety by its radioactivity as well as by the difference in mass. In disintegrating it emits a beta particle that can be interpreted as the transformation of one of the nuclear neutrons into a proton. When this occurs the nucleus has two positive charges, its atomic number is 2 , and it behaves chemically as helium. This element is stable and differs from the common variety of helium in that the nuclear mass is 3 instead of 4. Likewise, when two neutrons are added to the common helium nucleus the configuration is again unstable, a neutron transforms into a proton with the emission of a beta particle, thus producing a lithium nucleus of atomic number 3 and atomic mass 6 , which is stable. This series of transformations is illustrated in figure 4.

(b) Types of disintegration.-It is to be noted that in each case mentioned the transformation occurs with the emission of a beta particle, which results in a negligible change in the mass of the nucleus but which increases the nuclear charge by one unit. This process is typical of all beta-ray transformations. Although this process was discovered among elements of high atomic number occurring in nature, it is now known to occur throughout the periodic table. It is associated with the postulated property of the neutron by which it has a probability of transforming into a proton. This probability varies with the conditions under which the neutron exists, being very high for free neutrons and relatively low for those in the nuclei of stable atoms. 
UCLEAR

¿HARGE

0

NEUTRON

1
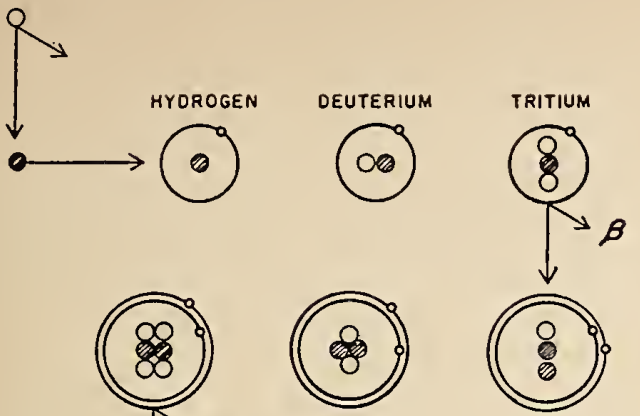

HYDROGEN

2
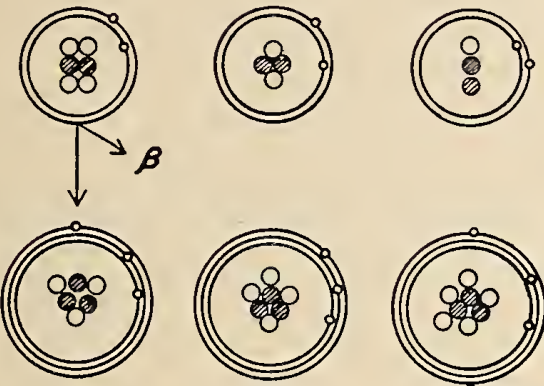

LITHIUM

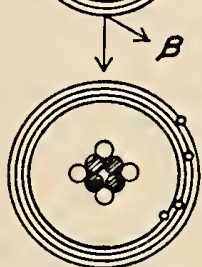

Figure 4. Chart showing the role of the neutron in artificial radioactivity.

Radioactive nuclei may also emit alpha particles in their transformation. When this occurs the nuclear charge decreases by 2 units and the atomic mass by 4 units. Alpha particle emission in connection with radioactive transformation is limited almost exclusively to elements of high atomic number. The emission of alpha particles can be explained qualitatively by assuming that in the heavier nuclei there is a tendency for two protons and two neutrons to become associated to form alpha particles as structural units of the nucleus. It is assumed that these alpha particles are held in the nucleus by strong forces of attraction for small internuclear distances. For greater distances the coulomb law of repulsion between electric charges of the same sign produces a repulsive force between the alpha particle and the rest of the nucleus. Under these conditions it has been shown from an application of the principles of wave mechanics that there is always a finite probability that the alpha particle will escape from the nucleus. Therefore, this representation results in a radioactive nucleus that will disintegrate by the emission of an alpha particle.

A considerable number of radioactive atoms disintegrate by emitting a positron. The nuclear process by which this occurs may be the transformation of a nuclear proton into a neutron. The new nucleus thus has the same mass as the parent but one less unit of charge. The same final result is obtained if a nuclear proton captures an orbital electron from the inner shell of the atom, assuming this process results also in the formation of a neutron. However, the only characteristic radiations emitted by the atom in this transformation are X-rays resulting from an outer orbital electron dropping in to fill the place of the one captured. A number of transformations by electron capture are known. They are usually associated with those which go partly by the emission of positrons and partly by orbital electron capture. The process is sometimes called $K$-capture, but it is not infrequent that an $L$-electron is captured instead.

In this introductory qualitative survey we must now consider the role of gamma rays and conversion electrons in the process of disintegration. The emission of gamma rays by the nucleus does not change either the mass significantly or the atomic number. Therefore, it can be regarded as a result of the reorganization of the newly formed nucleus. As can be expected from this view, gamma rays may accompany any type of nuclear disintegration, which is verified experimentally. However, not all types of disintegrating atoms emit gamma rays and the intensity and energy of the gamma rays vary widely from one type of atom to another. The intensity and energy of the gamma rays may be taken as a measure of the reorganization that takes place.

The conversion electrons have been identified as orbital electrons ejected by nuclear gamma rays and thus are distinguished from nuclear beta rays, although they have the same physical properties otherwise. If a gamma ray of energy $E$ is converted, it is to be expected that groups of electrons should be found with energies $E_{\gamma}-E_{K}, E_{\gamma}-E_{L}, E_{\gamma}-E_{M}$, where $E_{K}, E_{L}$, and $E_{M}$ are the binding energies of the electrons in the $K, L$, and $M$ levels. The experimental confirmation of this relation identifies the conversion electrons.

(c) Role of gamma rays.-In the process of formation of radioactive nuclei from stable forms, energy is added to the target nucleus. This may be accomplished by the capture in the stable nucleus of a neutron, deuteron, or alpha particle, or by the absorption of radiation. This process forms an intermediate nuclear form with a high degree of excitation, which frequently proceeds to radiate this energy in a number of gamma-ray quanta. This is the gamma radiation of the $n, \gamma$ process. Even when most of the energy of excitation is removed by the emission of another particle, the part remaining may be radiated as gamma rays. Another example is provided by those naturally radioactive isotopes that disintegrate by the emission of alpha particles. Frequently the product nucleus is left in an excited state that returns to the ground level by the emission of gamma rays. In such cases of nuclear excita- 
tion the time required for the excited nucleus to reach the ground state has been estimated to be of the order of $10^{-14}$ second. This reaction therefore has a very high probability of occur- rence as contrasted with the probability of the disintegration of the unstable nucleus in the ground state formed by the process of excitation and emission of gamma rays.

\section{DETECTION AND MEASUREMENT OF RADIOACTIVE RADIATIONS}

The principal general methods by which radioactive radiations can be detected and measured depend on their ability to ionize atoms either directly or by means of secondary radiations. The radiation from uranium was detected by Becquerel by use of photographic plates. Here the ionizing effects of the radiation render the compound of silver in the emulsion developable by chemical treatment, in much the same way that light reacts with the silver in ordinary photography. All types of radioactive radiation can be detected by this method, either directly or by means of secondary radiations.

When the ionization is produced in a gas, the electrical conductivity of the gas is increased in proportion to the degree of ionization. Thus by applying an electric field to the ionized volume of gas, a current is obtained proportional to the ionization, which in turn is proportional to the intensity of the radiation. This method may be used with modifications to measure all types of radioactive radiation. For example, although neutrons produce no ionization directly, they do eject protons from materials rich in hydrogen. The protons ionize the gas in proportion to the number of neutrons that produced them.

The various methods of counting radioactive radiations are also based chiefly on the ionization property of the radiations. In general, counting is accomplished by amplifying the pulse of ionization resulting from the passage of a single ionizing particle through the gas. This ionizing particle may be a constituent of the primary radiation, as an alpha or beta particle, or a secondary ionizing particle such as a photoelectron produced by a gamma ray, or a proton produced by a neutron. The amplification may be accomplished by electronic circuits where the initial ionization is sufficient to produce a pulse that can be amplified conveniently in this manner, or the amplification may take place largely in the ionized gas itself as in the Geiger-Müller tube counter.

Examples of methods occasionally employed that do not depend on ionization are: (1) the measurement of the charge transported by those radioactive radiations that consist of charged particles, and (2) the electron multiplier tube in which electrons are released from the electrodes by impact of the radiation.

\section{Photographic Emulsions}

For the quantitative measurement of radiation, photographic emulsions must be used under carefully controlled conditions such as are required for photometric measurements by photographic methods. The blackening of a photographic plate by light is given by the Schwarzschild formula

$$
D=f\left(I^{g} t\right) \text {, }
$$

where $I$ is the intensity of the light and $t$ the time of exposure. The logarithm form is

$$
D=f(q \log I+\log t) \text {. }
$$

The exponent $q$ is obtained by using two intensities $I_{1}$ and $I_{2}$ in exposure times $t_{1}$ and $t_{2}$, so that the same blackening is produced. Then

$$
q=\frac{\log t_{2}-\log t_{1}}{\log I_{1}-\log I_{2}} .
$$

It is found that $q$ is very close to unity for most intensities of light and times of exposure. For $q=1$ the Schwarzschild law becomes the Bunsen-Roscoe law

$$
D=f(I t) \text {, }
$$

so that for a given density the product $I \times t$ is a constant. In this relation $D$ is determined photometrically and is defined by the relation

$$
D=\log i_{0} / i \text {, }
$$

where $i_{0}$ is the intensity of the light incident on the developed photographic plate, and $i$ is the intensity of the light transmitted through the plate.

The Bunsen-Roscoe law is valid for exposure to alpha rays, at least for intensities of the ratio of $10: 1$. It also holds for both heterogeneous and homogeneous beta rays. Ellis and Wooster [2] have shown that the relation

$$
D=C \log (E / \tau+1),
$$

may be applied to exposures of beta rays, where $E$ is the energy of the incident beta rays, $C$ is a constant dependent on conditions of photographic development, and $\tau$ is a constant re- 
lated to the energy of the particles. This correction for the energy is not well known but has been assumed proportional to $\beta^{2}$ where $\beta=v / c$, the ratio of the velocity of the beta particle to that of light.

Investigations of the blackening produced by gamma rays show that the Bunsen-Roscoe law applies for intensities of the ratio of $100: 1$.

Another interesting application of photographic plates is the recording of the tracks of ionizing particles of considerable energy such as alpha particles, protons, and cosmic ray mesons. This method is successful only in special emulsions where chemical fog and other disturbing photographic effects are reduced to a minimum. Since the length of these tracks is very short, microscopic examination of the emulsion is required to measure them. From such measurements the energy of the radiation can be determined if the energy-range relation is known for the type of particle under observation.

\section{Crystals}

A few crystals have properties that make them suitable for detecting radiations from radioactive substances. Under appropriate conditions these crystals can be used for quantitative measurements. The classic example of the use of crystals is the visual scintillation counter developed by Rutherford and used in many important experiments in regard to the nature of the nucleus. This method depends on the ability of specially prepared zinc sulphide crystals to produce a small flash of light for each alpha particle that strikes it. A low-power microscope is required for visual observation and counting of the particles. This procedure has several obvious disadvantages, which include the variations of natural visual acuity of the observers, fatigue of the eye, limitation to low rates of counting resulting in long periods of observation for reasonable statistical accuracy. Hence, this laborious method has been replaced by electrical methods, such as electronic amplification of the ions produced by alpha particles.

In recent years crystals have assumed a revived importance in the field of radioactive measurements. This has come about in two distinctly different ways, one of which involves the counting of scintillations by means of photo-tubes and the other the detection of the electrical pulse produced within the crystal when radiation stimulates the crystal to release electrons in the conduction bands of the crystals.

Photo-electric scintillation counters are obvious extensions of the earlier visual scintillation counters. The introduction of the multiplier photo-tube and development of special luminescent substances have resulted in a revolutionary improvement in speed and sensitivity of counting. The photo-tube counters can be used for counting beta and gamma rays as well as alpha particles. The pulses produced are fast, so that high counting rates may be used without serious statistical losses. The most serious complication in their use is the natural "noise" in most photo-tubes, which may mask or interfere with the pulses produced by the scintillations. This noise can be reduced by refrigerating the phototube or by constructing photo-tubes of special design, which have a low noise level. Whereas zinc sulphide works well with photo-tubes for counting alpha particles and special phosphous for beta particles, crystals of napthalene or anthracene give best results with gamma rays. These organic crystals are quite transparent to their own fluorescent radiation and therefore may be used in thick layers which largely accounts for a sensitivity adequate for measurement of gamma radiation. The principle of the photo-tube scintillation counter is illustrated in figure $5 \mathrm{a}$.

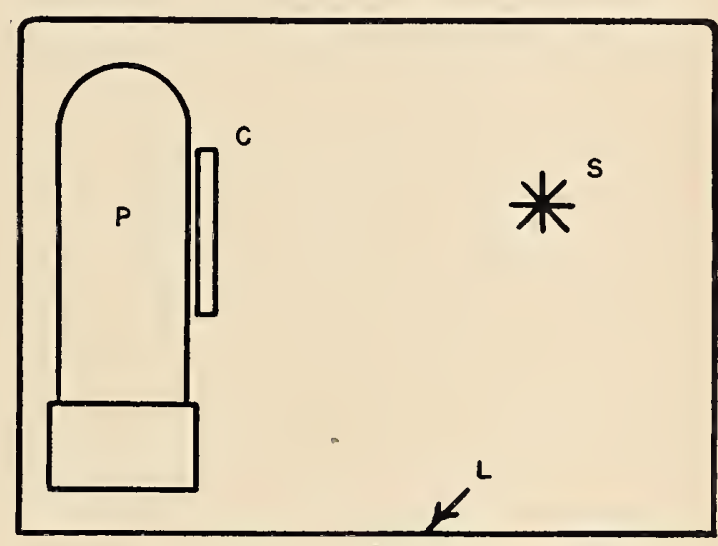

$\mathbf{a}$

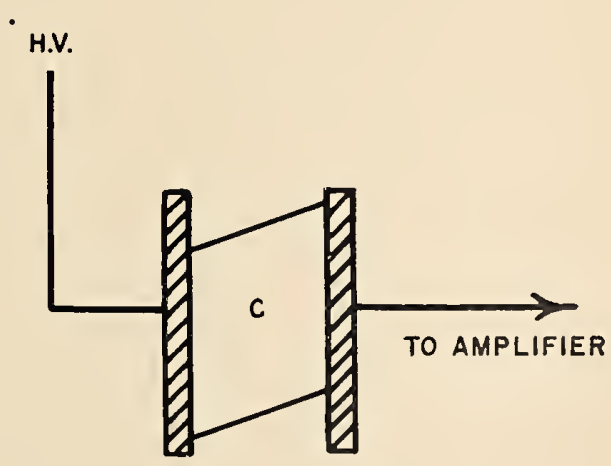

b

FIgURE 5. a, Multiplier phototube for counting scintillations from $\alpha, \beta, \lambda$-rays and fast neutrons. $P=$ photo-tube; $C=$ crystal, $S=$ source, $L=$ light shield. b, Crys$1,500 \mathrm{v})$. 
The pulse crystal counter was first developed by Van Heerden in a dissertation published in 1945 [3]. His successful experiments were made with crystals of silver chloride at liquid air temperatures. A number of other crystals have been found to possess the same properties. One of these, the diamond, can be operated at room temperature. The pulses produced by these crystals arise when an electron receives sufficient energy from the radiation to place it in the conduction band of the crystal. If an electric field is generated between two opposite faces of the crystal, the electrons thus released produce a displacement current. Consequently the counting property is based on phenomena essentially different from the photoelectric conduction observed in many crystals. Actually, to operate as a counter the crystal must be highly nonconductive in the ordinary sense, since conduction current will tend to interfere with, or mask the pulses. The size of the pulses depends on the number of electrons released and the distance that they travel in the crystal for the absorption of a single particle or quantum of radiation. These factors in turn depend on the nature and purity of the crystal, the energy of the incident radiation, and, to a lesser extent, upon the magnitude of the electrical field across the crystal. Due to the nature of the displacement current, intimate contact with the surfaces of the crystal are not required. For example, clamping a diamond between brass electrodes yields as good results as are obtained by depositing silver electrodes on the faces. Figure $5 \mathrm{~b}$ shows the essential arrangements for counting pulses from crystals. The pulses developed by most crystals are small and have a considerable range of -magnitude. Therefore a relatively high gain amplifier is required with a discriminator to reject all pulses below a certain level to eliminate the effects of background noise.

\section{Ionization of Gases}

Radioactive radiations may produce ions in gases in a variety of ways such as by impact of charged particles on the atoms of the gas or by the photoelectric removal of electrons from the atoms of the gas by electromagnetic radiation. Although the fundamental process consists in the removal of one or more electrons from atoms or molecules of the gas, electrons rarely persist unattached long enough to play an important role. They are taken up by neutral atoms in most gases to form negative ions of a mass comparable to that of the positive ions formed in the ionization process. Pure nitrogen and the noble gases are exceptions to this rule. The tendency for electrons to form negative ions in these gases is sufficiently small that negative ions consisting predominantly of elec- trons may be collected by an electric field. Although the charge carried by an ion is one or more elementary units, equal to the charge of an electron, the masses of ions may vary greatly depending on the gas or vapor in which they are formed, the way in which they are produced, and the rapidity with which they are collected.

a. Recombination.-Ions tend to return to the neutral state by recombination in which negative and positive ions share their difference" in charge to produce neutral atoms, so that in the absence of an electric field an equilibrium is soon established where the rate of recombination is equal to the rate at which ions are formed. The coefficient of recombination, $a$, is defined by the relation

$$
d n / d t=a n_{1} n_{2}
$$

where $n_{1}$ is the concentration of positive ions and $n_{2}$ of negative ions and $d n / d t$ is the rate at which ion pairs disappear per unit volume. When, as is usual, $n_{1}=n_{2}=n$ this relation becomes $d n / d t=-a n^{2}$. Experimentally $a$ has a value of $1.6 \times 10^{-6} \mathrm{~cm}^{3} \mathrm{sec}^{-1}$ for air at atmospheric pressure and room temperature.

b. Mobility of ions. - The mobility of an ion is defined as its velocity in centimeters per second in an electric field of $1 \frac{\mathrm{v}}{\mathrm{cm}}$. This motion of the ions constitutes an electrical current and, as in all cases, it represents the average rate of displacement of the carriers of charge due to the electric field. The mobility is characteristic of a definite type of ion but also depends on the nature, density, and temperature of the gas.

c. Electrical conductivity of a gas.-The specific conductivity, $L$, of a gas is given by

$$
L=n e\left(u_{1}+u_{2}\right) \text {, }
$$

where $n$ is the concentration of ion pairs, $e$ is the ionic charge, and $u_{1}$ and $u_{2}$ the mobilities of the positive and negative ions. The quantity $n$ is determined by the number of ions generated per unit time and volume, $q$, decreased by the loss of ions due to recombination.

In the absence of an electric field the conductivity attains a maximum value, $L_{0}$, where an equilibrium condition is set up such that the number of ions generated equals those lost by recombination so that

$$
q=a \dot{n}_{0}^{2} \text { or } n_{0}=\sqrt{q / a} \text {. }
$$

Therefore

$$
L_{0}=e\left(u_{1}+u_{2}\right) \sqrt{q / a} .
$$


On the application of an electric field the charge produced by ionization, $q e$, is balanced by loss due to recombination and to the current, $i$, which is set up. Hence

$$
q e=a n_{0}^{2} \mathrm{e}+\mathrm{i} \text { or } i=q e\left(1-\alpha n_{0}^{2} / q\right),
$$

where qe represents the maximum current for a given $q$ and is obtained when the ions are collected so rapidly that recombination may be neglected. Noting that

$$
\frac{L}{L_{0}}=\frac{n e\left(u_{1}+u_{2}\right)}{e\left(u_{1}=u_{2}\right) \sqrt{q / a}}=\frac{n}{\sqrt{q / a}}
$$

we can write

$$
\begin{aligned}
\frac{L^{2}}{L_{0}^{2}}=\frac{n^{2}}{q / \propto} & =a n^{2} / q=1-1+a n^{2} / q=1-\left(1-a n^{2} / q\right) \\
& =1-\frac{q e\left(1-a n^{2} / q\right)}{q e} .
\end{aligned}
$$

Therefore

$$
\frac{L}{L_{0}}=\sqrt{1-\frac{q e\left(1-a n^{2} / q\right)}{q e}} .
$$

But

so that

$$
q e\left(1-a n^{2} / q\right)=i
$$

$$
\frac{L}{L_{0}}=\sqrt{1-i / q e}=\sqrt{1-i / S},
$$

if we replace $q e$, which represents the saturation current, by $S$.

The voltage $V$, required for a current $i$ per unit volume is proportional to $i / L$, or to

$$
\frac{i}{L_{0} \sqrt{1-i / S}}
$$

Therefore

$$
V=k \frac{i}{L_{0} \sqrt{1-i / S}} .
$$

For values of $i$ small compared with $S$ this relation is very closely linear so that the current is proportional to the voltage. For larger values of $i$ the current rises more slowly with the increase of $V$ so that $i$ approaches $S$ asymptotically. This is illustrated in figure 6 , where the current $i$ is plotted as ordinates and the corresponding voltage, $V$, as abscissa for an arbitrary value, $S=10$. The plot $B$ is the initial portion of the curve $A$ on a larger scale of the curve $A$ showing linearity for small values of $i / S$.

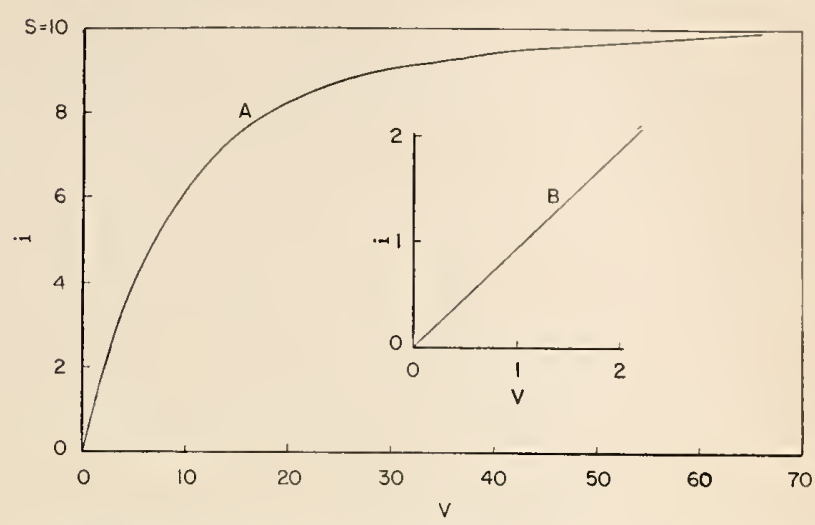

FIGURE 6. Variation of ionization current with voltage applied to the chamber.

Curve $A$ shows the gradual approach to the saturation value; curve $B$ is the initial portion of $A$ on an expanded scale show ing the linear relation between $i$ and $V$ in this region.

Although these calculated relations are only approximate because of the simplicity of the assumptions, they show the general behavior to be expected under experimental conditions.

Since

$$
V=k \frac{S}{L_{0}} \frac{i / S}{\sqrt{1-i / S}}
$$

the value of $V$ for a predetermined value of the degree of saturation, $i / S$, is proportional to

$$
\frac{S}{L_{0}}=\frac{q e}{e\left(u_{1}+u_{2}\right) \sqrt{q / a}}=\frac{\sqrt{q a}}{u_{1}+u_{2}}
$$

so that the voltage is proportional to $\sqrt{q}$ and inversely proportional to the mobilities of the ions. In general

$$
V=\frac{i / S l^{2} \sqrt{q / \mathrm{a}}}{u_{1}+u_{2}},
$$

where $l$ is the distance between the electrodes. For a given intensity of radiation, the saturation voltage decreases rapidly with a decrease of pressure of the gas. This is caused by (1) the decrease in the production of ions and (2) an increase in their mobility, since the ionization is proportional to the pressure and the mobility inversely proportional to it. Whereas in ionization chambers of moderate volume at atmospheric pressure it is possible to obtain a satisfactory approach to the saturation cur- 
rent for a moderate ionization by beta and gamma rays, this is comparatively difficult for ionization produced by alpha rays. This arises from the dense local ionization produced by alpha rays and the resultant rapid recombination.

\section{Measurement of Charge Transported by Alpha and Beta Rays}

The intensity of alpha and beta rays can be measured under suitable conditions by determining the rate at which an insulated receiver accumulates charge, or the potential developed across a high resistance connected to the receiver. Such measurements require that the space between the sources and receiver be evacuated to eliminate ionization. The method is practicable only for relatively strong sources, since $10^{5}$ beta particles per second is equivalent to $1.6 \times 10^{-14} \mathrm{amp}$. To secure a measurement of the total number of particles emitted by a source, it is more convenient to connect the source through a high resistance to ground, with the source located at the center of a well evacuated chamber of considerable volume. The steady potential, in volts, of the source measured under these conditions will be

$$
V=R\left(1.6 \times 10^{-19}\right) N,
$$

where $N$ is the number of particles emitted per second. From this relation we have

$$
N=\frac{V}{R\left(1.6 \times 10^{-19}\right)}
$$

\section{Counting Methods}

The rate at which individual particles or photons, which constitute the various types of radioactive radiations, are emitted may be determined by counting the individual constituents, such as alpha particles, beta particles, protons, positrons, or neutrons. The various methods for doing this all depend on the ionizing properties of the particles themselves, or of the secondary radiations produced by them. In the practical applications of all of these methods only some fraction of the total radiation is counted. This fraction is determined by the geometrical arrangement and, in some cases, by absorption of windows or walls of the detecting chamber, as well as the intrinsic effciency of the detector. For absolute quantitative measurements, therefore, a correction factor must be ascertained for each counting device. Three general types of counting methods can be distinguished, depending on the method of amplification of the ionization produced by the primary event in the detector.

\section{Ionization Chambers}

In principle, the most simple is the method where the primary ions for each particle are collected on an electrode and the resulting potential pulse is amplified by some type of vacuum tube amplifier, such as a capacity-resistance coupled linear amplifier. The collecting potential applied to the electrodes of the chamber in which the primary ionization is produced is only sufficient to collect all ions rapidly without permitting any to gain sufficient energy to produce secondary ions. This method can be applied only to those particle radiations that produce a sufficient number of primary ions to

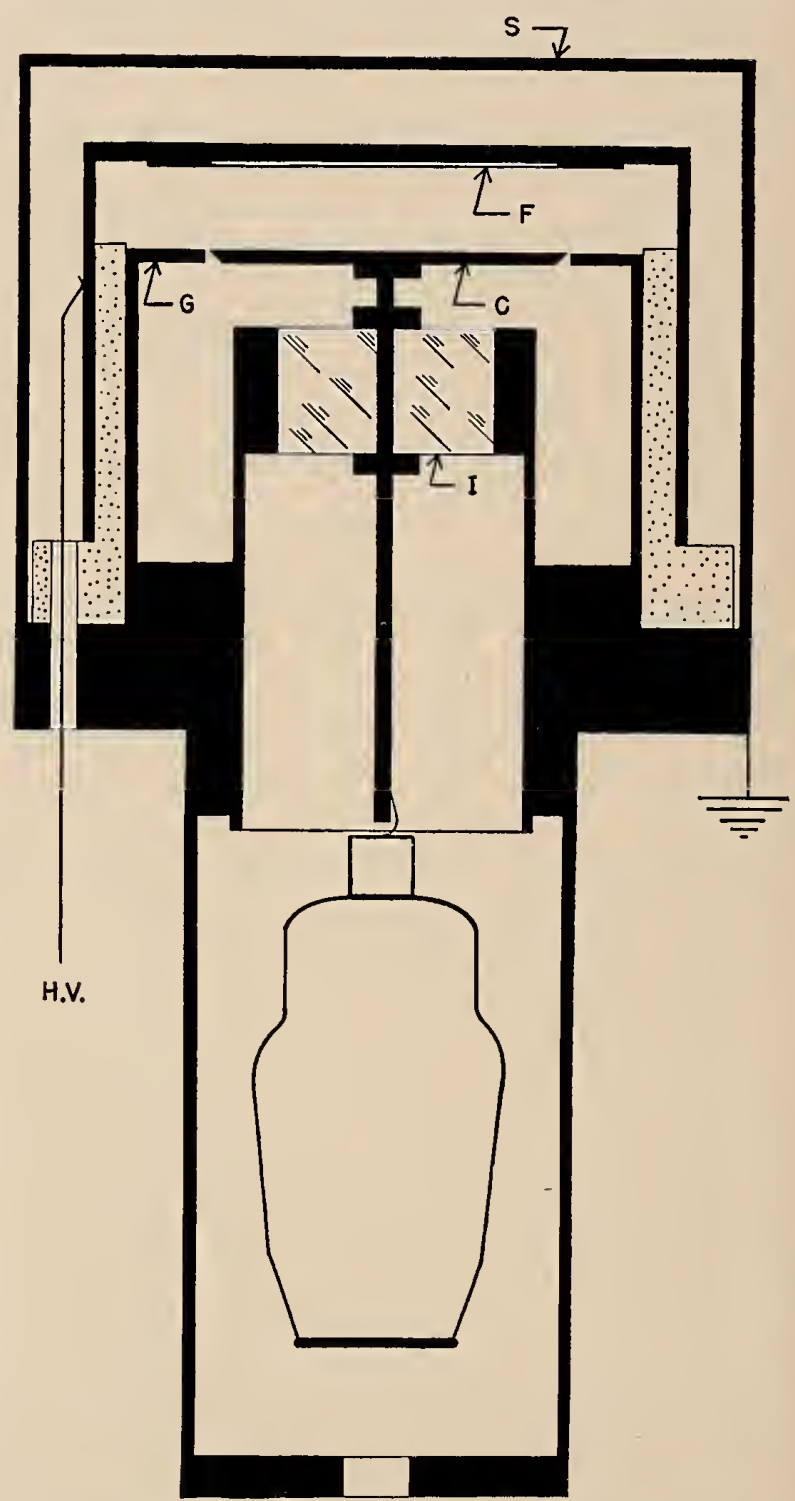

Figure 7. Paralled plate chamber for counting ion pulses from individual alpha particles. $S=$ shield; $F=$ source of alpha particles; $C=$ ion-collecting elec-
trode; $G=$ guard ring; $I=$ insulator. 


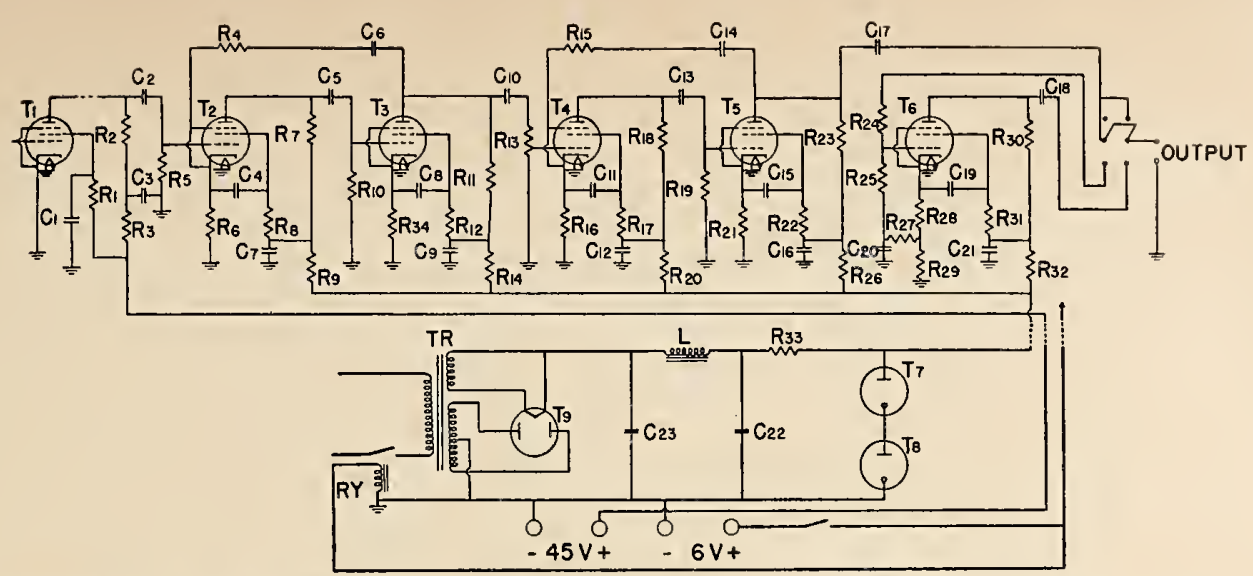

FIGURE 8. Linear amplifier for use with chamber for counting ion pulses.

$R_{1}, R_{8}, R_{12}, R_{17}, R_{22}, R_{31}=1.5$ megohms; $R_{2}=100,000$ ohms WW. $R_{3}=50,000$ ohms WW. $R_{4}, R_{5}, R_{10}, R_{15}$ $R_{10}, R_{24}=1$ megohm; $R_{8}, R_{34}, R_{18}, R_{21}=2,000$ ohms; $R_{9}, R_{27}=100,000$ ohms; $R_{7}, R_{13}, R_{18}, R_{23}, R_{25}$ $R_{2 n}=250,000$ ohms; $R_{14}, R_{20}, R_{28}, R_{32}=50,000$ ohms; $R_{13}, 1$ megohm volume control; $R_{28}=1,000$ ohms: $K_{29}=10,000$ ohms; $R_{33}=15,000$ ohms; $L=$ filter choke; $C_{2}=0.0005 \mathrm{mfd}$ mica; $C_{1}, C_{3}, C_{0}, C_{0}, C_{12}, C_{14}$ $C_{18}, C_{20}, C_{21}=2 \mathrm{mfd} ; C_{4}, C_{8}, C_{13}, C_{15}, C_{19}=0.05 \mathrm{mfd} ; C_{7}, C_{22}, C_{23}=8 \mathrm{mfd} ; C_{5}, C_{10}, C_{13}, C_{17}, C_{18}=0.1$ mid; $T_{1}=$ type $38 ; T_{2}, T_{3}, T_{4}, T_{6}, T_{8}=6 \mathrm{~J} 7 ; T_{8}=$ type $80 ; T_{7}, T_{8},=$ VR-150; $R Y=$ relay; $T R$, trans former, 700 volt secondary C.T.

produce a pulse that can be amplified by electronic circuits without interference from the background disturbances present in amplifiers of very high gain. Alpha particles, slow protons, and fission particles have been successfully counted by this method.

A typical example of an arrangement for counting alpha particles by means of the primary ionization is shown in figure 7 , which shows a parallel plate ionization chamber with one electrode connected directly to the grid of the first vacuum tube of the linear amplifier. A potential of from 500 to $1,000 \mathrm{v}$ is sufficient to collect the primary ions. Whereas such chambers can be operated with air in the chamber, the pulses are larger and more sharp with pure nitrogen, or argon, with the collecting electrode at positive potential. The initial rise of potential is very rapid under these circumstances, since the negative ions in these gases are mainly electrons with a much higher mobility than ions of gases that form heavy negative ions as a result of attachment of electrons to atoms or molecules of the gas.

Figure 8 shows a diagram of a linear amplifier with negative feed-back, described by Waddell [4], which is suitable for use with various chambers of the type under discussion.

In addition to counting the particles, this method also distinguishes between particles of different specific or total ionization depending on the design of the chamber. Each pulse is proportional to the number of primary ions collected, so that the alpha particles of different ranges can be distinguished and counted separately. Alpha particles can also be distinguish-

2 An analyzer that uses hard vacuum tubes throughout, discarding thyratrons, is described by H. F. Freundlich, E. P. Hincks, and W. K. Ozeroff (Rev. Sci. Instr. 18, 90 1947). ed from fission particles, and fission particles of various energies can be differentiated.

a. Differential pulse analyzer.-The usefulness of the method in counting alpha particles of various ranges from a complex source such as ordinary uranium, or in determining the distribution of energy of fission particles, is increased by use of a differential pulse analyzer ${ }^{2}$. This is an electronic circuit with various channels, each of which records only those pulses that are of sufficient size to actuate that particular channel; smaller or larger pulses are not recorded by the channel. One form is shown schematically in figure 9 .

The three initial channels only are shown for simplicity. The amplified pulses are fed into the terminal labelled pulse input. All pulses that provide a signal at the grid of the thyratron $T_{1}$ greater than the bias $-C_{1}$ will tend to fire this thyratron. However, a pulse that is large enough to fire the thyratron $T_{2}$, but fails to fire $T_{3}$, will feed a signal from the cathode that cancels the signal from the plate of $T_{1}$. Therefore $T_{1}$ transmits only those pulses large enough to overcome the bias $-C_{1}$ but not large enough to fire $T_{2}$, and a pulse results at $A_{1}$. In this way a dozen or more channels may be connected as shown with a graduated series of negative biases, each channel responding only to those pulses large enough to fire its thyratron and failing to respond to smaller or larger pulses. Thus in operation pulses produced by alpha particles of differing total ionization, or range, are recorded in the appropriate channel. By recording the pulses from $A_{1}, A_{2}, A_{3}$, the number of particles of various ranges are obtained concurrently. A typical curve obtained from natural uranium is shown in figure 10 , showing the peaks due to particles from $U I$ and $U I I$ with a suggestion of the peak produced by particles from AcU. 


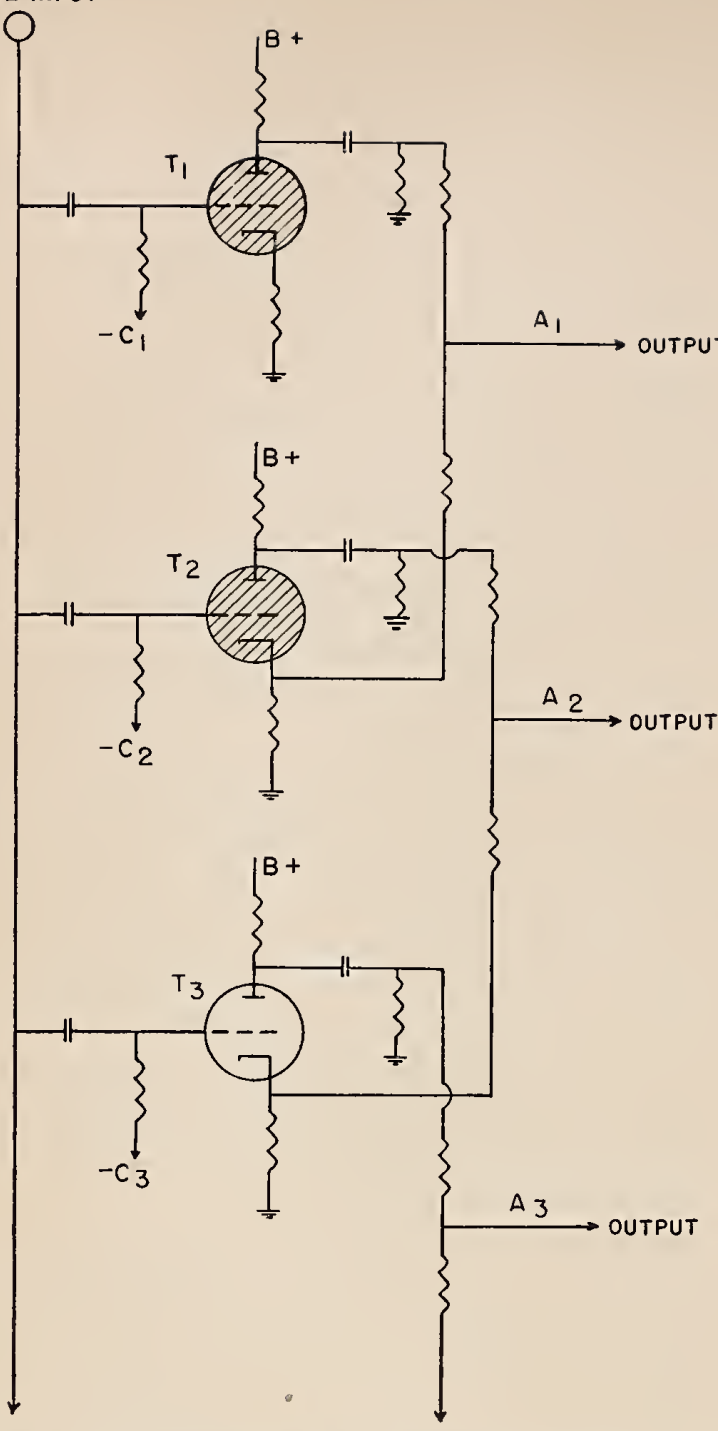

Figure 9. Differential pulse analyzer. voltage.

$T_{1}, T_{2}, T_{3}=$ thyratrons, $-C_{1},-C_{2},-C_{3}$ increasing negative bias

\section{Proportional Counters}

The second method involves an amplification of the primary ionization in the gas of the counting chamber by providing a potential gradient in which the primary ions acquire sufficient energy to produce a limited number of secondary ions. Since under these conditions the number of secondary ions can be made more or less proportional to the number of primary ions, these devices are called proportional counters. The high potential gradient required is obtained by using a wire or small sphere as the anode mounted on the axis of a cylindrical tube forming the cathode. Potentials ranging from 1,500 to $4,000 \mathrm{v}$ are required to operate

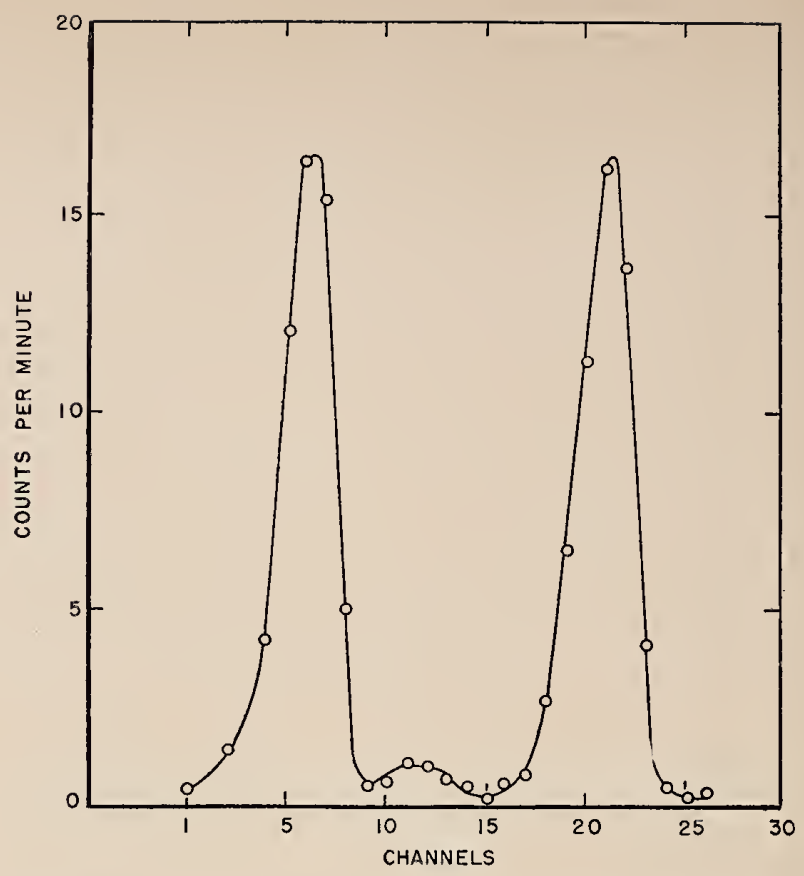

FIgURE 10. Distribution of number of pulses at various voltages (channels) for ion pulses produced by alpha particles from a sample of natural uranium.

proportional counters, depending on the nature and pressure of the gas in the counter.

The principle by which proportional counters operate can be illustrated by means of figure 11, which shows diagrammatically the electrical

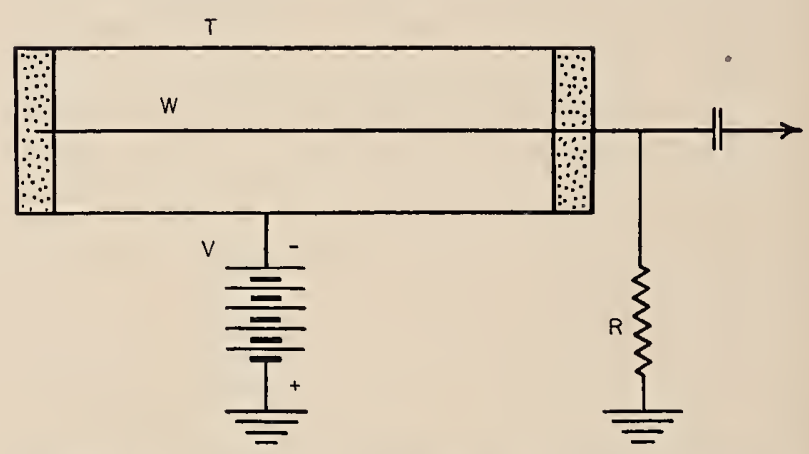

FIgURE 11. Schematic diagram of a Geiger counter, which may be operated either in the proportional region or the Geiger-Müller region.

$T=$ metal tube; $W=$ central wire; $V=$ battery; $R=$ resistor.

circuit for the central-wire type counter. The wire, $W$, is held, ordinarily, at a positive potential with respect to the tube, $T$, by a source of voltage, $V$, connected to the wire through the resistor, $R$. When the potential $V$ is sufficiently high, an electric field is created in the neighborhood of the wire in which electrons after release from the cathode can acquire 
energy sufficient to ionize neutral atoms on their first impact. At this point the phenomenon of gas amplification of the ionization current begins to appear. As the potential $V$ is increased, the volume about the wire, where additional ions are produced, and the number of such ions per primary electron also increase. The instantaneous pulse produced on the wire, $d V$, is given by $A m c /(C)$, where $n$ is the number of primary electrons, $A$ is the number of ion pairs produced by each on its path to the central wire, $e$ is the elementary charge, and $C$ the total capacity of the circuit connected to the wire. Thus $A$ represents the amplification in the gas, and a counter is strictly a proportional counter only when $A$ is constant. The relation between ionization chambers, proportional counters, and Geiger-Müller counters is illustrated by the diagram in figure 12 , where the pulse size is plotted

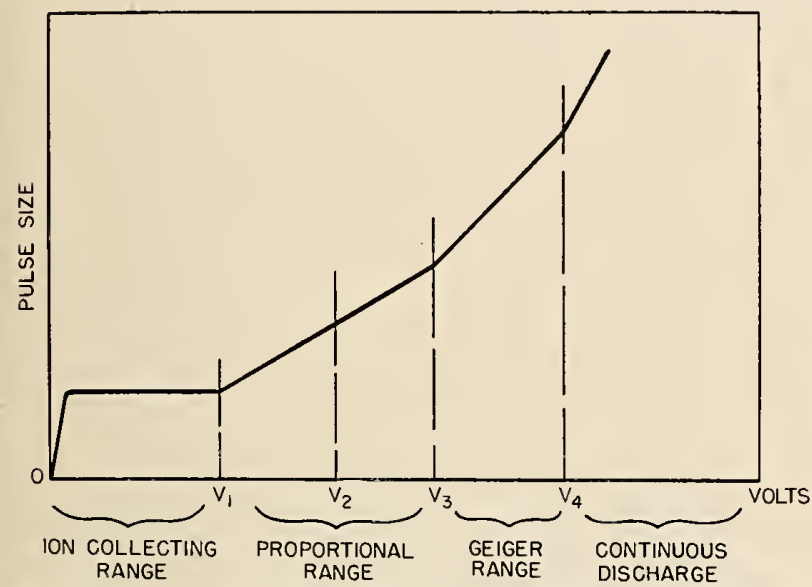

FIGURE 12. Diagram showing variation of pulse size with voltage applied to a counter of the general design shown in figure 11.

against the voltage applied to the counter. In the range from 0 to $V_{1}$ the counter tube behaves as an ionization chamber. There is an initial short range where the number of ions collected is proportional to the voltage, followed by a region where the pulse size is constant as the voltage increases. This is the saturation region of an ionization chamber. Beyond $V_{1}$, the primary ions produced by the radiation entering the counter tube acquire sufficient energy from the electron field to produce secondary impact ionization of the gas, which also contributes to the size of the pulse. For voltages between $V_{1}$ and $V_{2}$, the number of secondary ions is proportional to the number of primary ions making this the true proportional region. In going from $V_{2}$ to $V_{3}$, a transition occurs from the proportional range to the Geiger-Müller range in which any primary ionization tends to produce a pulse of the same size, regardless of the number of primary ions. Finally, at $V_{4}$, a condition is reached where all pulses at a given voltage are of the same size. This condition is main- tained until $V_{5}$ is reached. Here the counter breaks into continuous discharge.

To operate as a counter, that is produce one electrical pulse on the wire for each primary ionizing particle entering the counter, the cumulative ionization must cease when the negative ions resulting from the primary ionization all reach the wire. The abrupt cessation of this process is brought about by three principal factors; the geometrical form of the counter, the low mobility of the positive ions produced, and the direction in which the negative ions, principally electrons, travel.

The high electric field, as well as the nature of the gas in some cases, reduces the recombination of ions in the counter to a low value. Therefore, as a result of their low mobility, the heavy positive ions remain in the gas of the counter very near the position where they were formed, during the short time in which the electrons reach the wire. Most of these positive ions are in a narrow sheath very near the wire because of the form of the field. This produces an almost field-free space between this sheath and the counter tube, representing the major volume of the counter. Therefore, the first avalanche is interrupted since no electrons in this volume of low potential gradient can acquire energy to produce ionization, and consequently no electrons are released in the region. Not only has the counting action been interrupted to give an abrupt pulse but the counter is insensitive to the arrival of another particle during the short interval required for this ion sheath to travel to the tube and thus restore the electric field to its original state. This interval of the order of $10^{-4}$ second has been called the "deadtime" of the counter.

Proportional counters have been used to count alpha particles, protons, beta particles, fission particles, and neutrons by using either a gas that reacts with neutrons to emit alpha particles, such as $B F_{3}$, or by coating the walls of the counter with boron. The resolving time of these counters can be decreased by use of special gases, such as methane. The amplifiers required need only have a moderate gain; two stages of pulse amplification with feedback plus a coupling stage to a scaler, or differential pulse analyzer, are usually adequate.

\section{Geiger-Müller Counters}

The Geiger-Müller counter, although similar in construction to the wire-type proportional counter, (figure 11), operates at lower gas pressures. For best operation it requires special gases or mixtures of gases and vapors, and it delivers pulses of uniform size regardless of the number of primary ions formed by the radiation entering it. The lower pressure increases the mobility of the electrons, so that 
Inuch more gas amplification is produced. As a result of the higher mobilities these counters do not produce sharply defined pulses by means of the space charge action of the positive ion sheath alone. Additional quenching action is required. Since in the early development of these counters this was accomplished by the use of a high value of the resistor, $R$, in figure 11 , this resulted in the counter becoming inoperative for the relatively long periods required for the electric field to be reestablished, corresponding to the long-time constant represented by $R C$. Electronic devices to accomplish the quenching by suddenly removing the potential for a short interval to allow recombination to remove all ions have been developed. However the commonly preferred solution to this problem is the self-quenching counter developed by Trost [5].

Selfquenching is obtained by filling the counter with a mixture of organic vapor, such as alcohol, with a gas of low electron attachment, such as argon. The function of the organic vapor or gas is mainly to absorb photons that have sufficient energy to release electrons in the gas and from the walls of the counter. It also reduces secondary emission of electrons from the walls of the tube produced by the arrival of the positive ions.

Therefore, in the Geiger-Müller counter the primary ions produced by the penetration of an ionizing particle, or photon, or other radiation into the effective volume of the counter generates a saturation amount of ionization by impact, so that all pulses are uniform regardless of the number of ion pairs produced by the primary agent. By the combination of the effects of the ion sheath and the quenching mixture the counting process is abruptly terminated. Finally, the positive ion sheath moves out to the walls of the tube and its ions are collected, which restores the counter to a condition where it will respond to the entry of an ionizing agent.

The characteristics of a good Geiger-Müller counter include a reasonably low and stable threshold, (that is the voltage at which counting begins) and a rapid rise in sensitivity as the voltage is increased above the threshold to a plateau where the counting rate of the counter for a steady source of radiation rises very slowly. This plateau may extend to several hundred volts and have a slope of the order of 0.01 percent/v. The flatness and length of this plateau principally determine the excellence of the counter. A typical characteristic curve is shown in figure 13, where the counting rate is plotted against the voltage applied to the counter for a constant source of radiation. $V_{0}$ is the threshold, $V$ is the operating voltage. The difference between these voltages is the over voltage, which is equal to the voltage of the pulse on the wire for each count. Beyond the plateau, the counting rate begins to rise rather sharply. Self-quenching counters should never be operated in this region since they rapidly deteriorate under such treatment and may not recover their original characteristics. It has been found that the threshold voltage of vapor type counters tends to shift with temperature and operating conditions. The whole plateau then shifts with the threshold voltage. Consequently, it is customary to check the threshold voltage at frequent intervals and change the operating voltage by an amount corresponding to the change in the threshold. This precaution is particularly important for counters with short or comparatively steep plateaus.

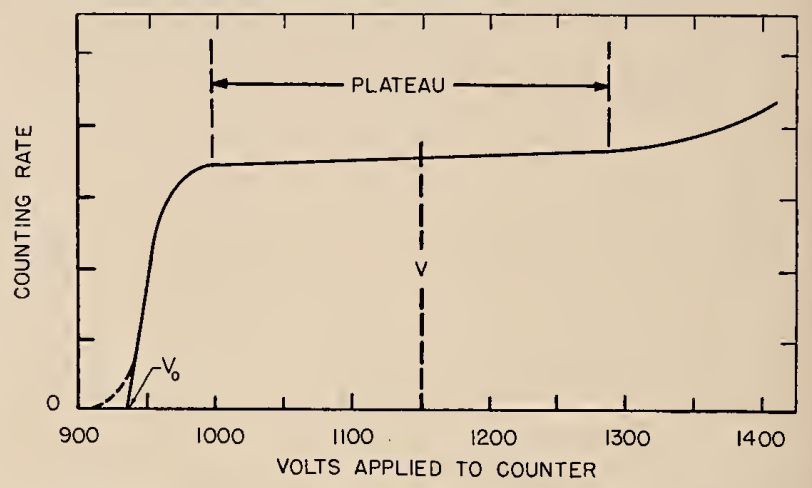

Figure 13. Curve showing plateau of Geiger-Müller counter.

$V=$ operating voltage, $V_{0}=$ threshoid voitage.

The plateau of a Geiger-Müller counter at the low-voltage end actually merges into a region where it behaves somewhat as a proportional counter. Therefore with sufficient amplification, pulses can be detected below $V_{0}$ as indicated by the dotted curve in figure 13 . However, it is customary to define $V_{0}$ by extrapolating the steep rise of the characteristic back to zero count. This gives a value scarcely distinguishable from that determined by ascertaining the voltage at which pulses are detected by an amplifier of moderate sensitivity.

The Geiger-Müller counter is sensitive to a single ion pair produced in the effective volume. Therefore it may be used to detect and measure any radiation that produces, directly or indirectly, such an effect.

It is used principally for measurement of beta and gamma rays and with appropriate modifications for the measurement of neutrons. The intrinsic efficiency of a Geiger-Müller counter, that is the proportion of the quanta traversing the effective volume that is detected, depends on the nature of the radiation. Practically every beta ray is detected, but only a small percentage of the gamma-ray photons are counted.

a. Gamma-ray counters.-For measurements of gamma rays the tube is made of moderately 
thick metal to increase the number of photo or compton electrons that are released from the tube and produce the primary ionization. The intrinsic efficiency of gamma counters for gamma rays below about $1.5 \mathrm{Mev}$ can be increased considerably by using tubes of heavy metals that increase the probability of photoelectric conversion of the gamma rays. A typical gamma-ray counter consists of a metal tube sealed in a glass envelope with the central wire supported by wire electrodes sealed through the ends of the glass tube. When evacuated and filled at the proper pressure with a selfquenching mixture, the filled tube is sealed off. Since the absorption of gamma rays by the glass wall is small, this arrangement is satisfactory.
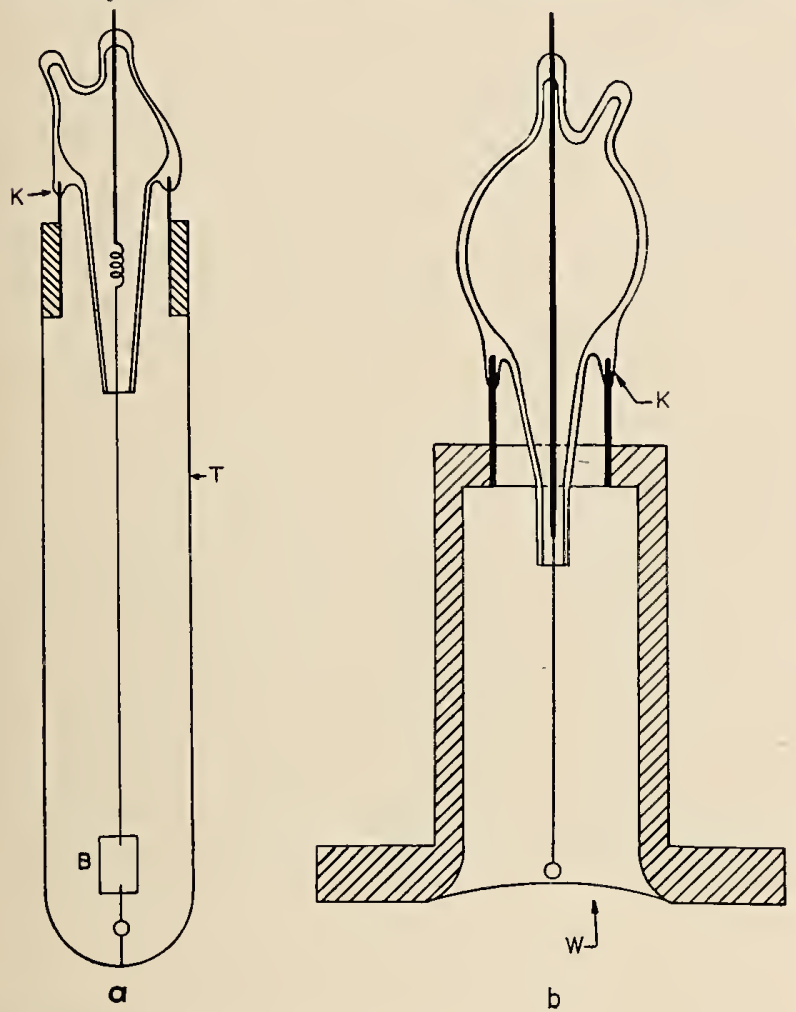

Figure 14. Beta-ray counters.

a. Beta-ray Geiger-Müller counter constructed with a thina. wovar seal. b, Bell-type beta-ray counter with thin circular
Koval mica window. " $W=$ Window; $K=$ Kovar seal.

b. Beta-ray counters.-The walls of GeigerMüller counters used for measuring beta rays must either be very thin, or special windows must be provided to permit this easily absorbed radiation to enter. For beta rays of very low energy, the counter must be directly open to the source. There are three common types of beta ray counters: (1) a thin glass envelope with a thin metallic coating forming the tube deposited on the inside; (2) aluminum tubes with wall thickness of 0.004 to 0.006 in., with the central wire supported by insulators, so that the aluminum tube forms both the envelope and the tube of the counter, shown in figure 14, a; (3) mica-window counters most commonly of the bell type, as shown in figure 14, b.

Mica-window or bell-type counters having a window thickness corresponding to $4.5 \mathrm{mg} / \mathrm{cm}^{2}$ are used successfully on apertures approximately 1 in. in diameter. Where larger apertures or thinner windows are required, special designs and considerable skill are required in obtaining windows that will withstand evacuation required in the process of filling. The importance of counters of this type has stimulated considerable effort to produce more satisfactory counter's.

c. Correction for recovery time in counters. -It has been demonstrated that all types of Geiger-Müller counters have a definite interval, following each response to radiation, in which the counter is insensitive. This effect has been investigated by Stever, who calls the insensitive interval the "dead time."

For random pulses, as occur in a counter, if $\bar{t}$ is the average time between particles, the average number per second $\bar{n}$ is equal to $1 / \bar{t}$. If the dead time is $t_{d}$, then for each second the counter is dead for $n t_{d}$ part of the time, or the counter is sensitive for $1-\bar{n} t_{d}$ fraction of the time. The true counting rate $n_{t}$, which would be obtained if the counter were continuously sensitive is related to the observed counting rate, $n_{0}$ as follows

$$
n_{t}=\frac{n_{0}}{1-\bar{n} t_{d}}
$$

When the deadtime is relatively short $\bar{n}$ may be assumed equal to $n_{0}$ in the above relation. To illustrate the importance of this correction to counting rates and therefore the necessity of knowing at least the approximate value of the deadtime of a counter, let us assume that the observed average counting rate is $200 / \mathrm{sec}$ for a counter with a deadtime of $1.5 \times 10^{-4} \mathrm{sec}$. The true number is obtained from

$$
n_{+}=\frac{200}{1-200\left(1.5 \times 10^{-4}\right)}=\frac{200}{0.97}=206 \text {. }
$$

If the counter had a deadtime of $5 \times 10^{-4} \mathrm{sec}$, we would have

$$
n_{t}=\frac{200}{1-200\left(5 \times 10^{-4}\right)}=\frac{200}{0.90}=222 .
$$

Statistical errors in counting are evaluated in terms of the fluctuations in the average number of random events per unit time. We have seen from eq. 24 that the standard deviation $E$, 
is given by $\pm \sqrt{Z}$, where $Z$ is the total number of events observed. Similarly here the standard deviation is given by $\pm \sqrt{N}$, where $N$ represents the total number of particles or pulses recorded in a particular set of observations. This standard deviation means that the true total number $N_{t}$ is approximately twice as likely to lie within the value $N_{t} \pm \sqrt{N}$ as outside of it.

In the case of rate meter circuits the standard deviation of a particular observation depends on the time constant of the condenser $C$, and resistor $R$, used to accumulate the rectified pulses, sometimes called the "tank" circuit. Schiff and Evans [6] have shown that it is given by $(2 n R C)^{-1 / 2}$, where $n$ is the average counting rate.

The standard deviation when a background is present is determined from the background count and from the count obtained from a source plus the background. The standard deviation for the combination is $\pm\left(n_{1}+n_{2}\right)^{1 / 2}$, where $n_{1}$ is the background count alone, and $n_{2}$ is the observed count with a source. As $n_{1}$ approaches $n_{2}$, the relative uncertainty in the value of $n_{2}-n_{1}$, the effect of the radiation, increases. Therefore it is desirable to reduce the background effect in counters when radiations of low intensity are to be measured.

\section{Electron Multiplier}

When electrons or positive ions strike a positive electrode in a vacuum tube, secondary electrons are ejected from the electrode. Under favorable conditions as many as four secondaries may be ejected by the primary ion. An electron multiplier tube contains a number of electrodes, usually about 12 , in which the secondary electrons are electrically focussed to impinge on the next successive electrode. The electrons from the final electrode fall on a collector that is connected to some type of amplifying and indicator circuit. Assuming an efficiency of 4 secondaries per electrode, a 12 electrode tube produces approximately $2 \times 10^{7}$ electrons per primary ion or electron incident on the initial electrode. This amplification is sufficient to increase the potential of a receiving electrode of small capacity by a few hundredths of a volt. Factors affecting the design of these tubes are discussed by J. R. Pierce [7].

These tubes must be operated with the interior evacuated to pressures of the order of $10^{-6} \mathrm{~mm} \mathrm{Hg}$. The electrode surfaces are coated with $B e$ to provide desirable characteristics for multiplier action, such as large work function for stability and a high ratio of secondary to primary electrons.

A diagram of electrodes and connections is shown in figure 15. The value of $V$ is usually about $300 \mathrm{v}$, and a secondary ion factor ap-

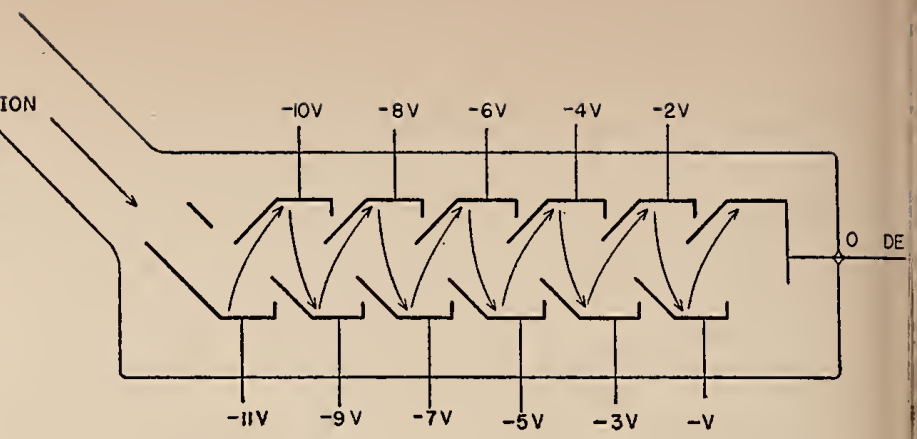

Figure 15. Diagram of electron multiplier tube.

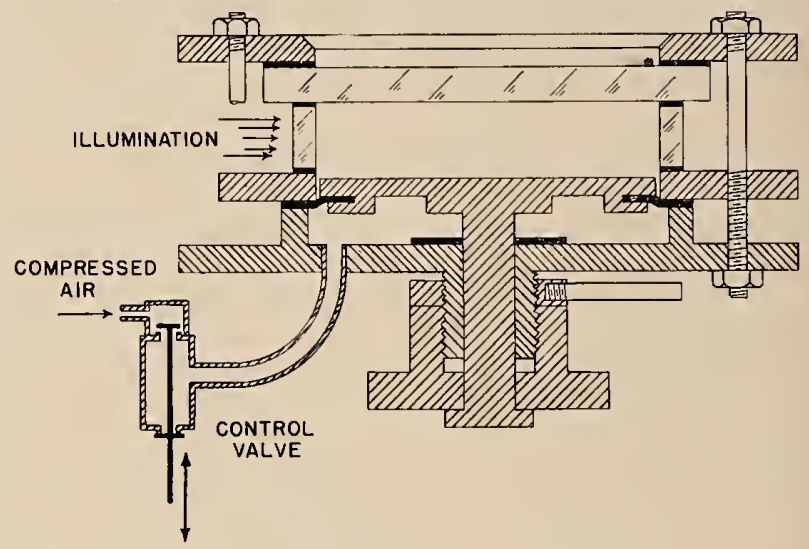

FIgURE 16. Cross section of cloud chamber.

proaching 4 can be obtained. The multiplier may be used with any type of radiation that releases electrons when striking the initial electrode. It is particularly useful in detecting alpha particles and protons, as well as positive ions in a mass spectrometer. It has an efficiency for gamma rays of the order of $10^{-4}$. Whereas alpha particles produce approximately $5 \times 10^{6}$ electrons at the collecting electrode (anode), gamma rays give pulses per photon of approximately one tenth this size.

\section{Wilson Cloud Chamber}

This device, originated by C. T. R. Wilson, makes use of the strong tendency of water vapor to condense on ions. The cloud chamber is a device to produce an atmosphere supersaturated with water vapor at the instant the ionization particle traverses it. The vapor condenses on the trail of ions and renders them visible under strong illumination. Alpha particles, protons, beta particles, fission products, and mesons may all be detected in this way. Recoil nuclei from neutrons and other particles will also produce tracks. A diagrammatic sketch of a typical cloud chamber is shown in figure 16. It consists essentially of a closed volume above a piston containing air or other gas at the desired pressure and saturated with water vapor. Mechanical or pneumatic arrange- 
ments are provided to move this piston abruptly a definite distance to produce an increase of the volume, and this produces a degree of supersaturation of the water vapor present depending on the distance through which the piston moves. At the same time the particles are permitted to enter the chamber to produce the tracks. If the ratio of the new volume to the original is greater than about 1.3, general condensation will occur. Therefore, means are provided for adjusting this expansion ratio to obtain tracks that are sharply defined. The pressure of stray ions, or those remaining from preceding operations, will tend also to produce a general cloud. To obviate this, an electric field is applied to the chamber between the upper glass plate and the piston to sweep out such ions previous to the expansion. Tracks are viewed, or for precise measurements photographed through the glass plate with strong illumination through the walls of the glass cylinder forming the vertical sides of the chamber.

Since only a small number of individually distinct tracks can be obtained for each expansion, the Wilson chamber is a relatively insensitive device. To obtain data free from large statistical fluctuations, numerous expansions must be made using the same experimental arrangement and a photograph taken of each result. Since the temperature of the chamber must be nearly the same at the initiation of each expansion, sufficient time must elapse between each operation to insure that temperature equilibrium has been restored. For effective use a cloud chamber must operate automatically with a device to control the various operations in a precise cycle.

The usefulness of this chamber is greatly increased by the application of a magnetic field perpendicular to the face of the piston. The curvature of the tracks produced in a magnetic field of known intensity gives valuable information regarding the nature of the particle producing the track.

The Wilson chamber is effective in. determining ranges of particles, in studying the effects of their collisions with other nuclei, and particularly in identifying the nature of the products where such collisions result in disintegration of the nuclei concerned. It is particularly valuable in studying new types of radiation of unknown properties and in this role has been of great use in studying the various components of cosmic radiation. Its chief disadvantage arises from the large number of photographs required to obtain accurate results or to detect and measure rare events.

\section{Calorimetric Methods of Measuring Radioactive Radiations}

The radiations from radioactive substances have been measured by measuring the heat de-

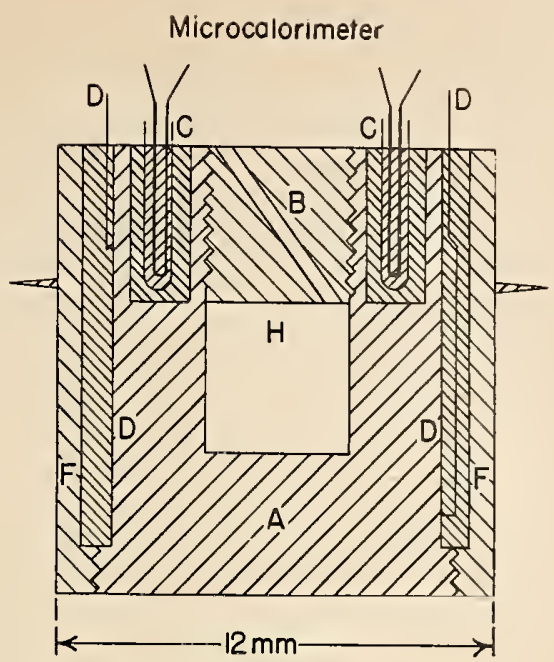

a
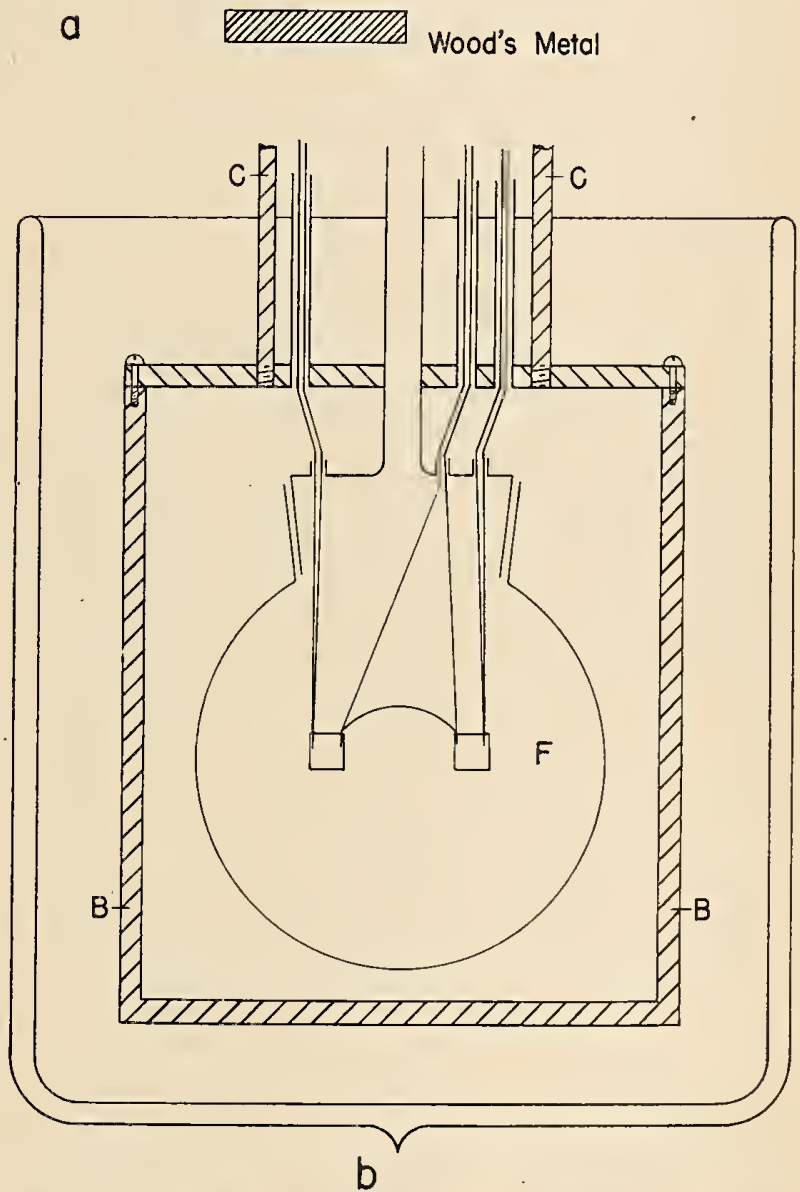

FIgURe 17. Microcalorimeter for measuring radioactive energy.

a, $A=$ copper cylinder; $B=$ copper plug; $C, C=$ thermocouples; i), $D=$ heating coils; $F, F=$ outer copper envelope. $b, B, B=$ brass box; $C, C=$ metal supports.

veloped when these radiations are absorbed. Since the radiation to be measured must be completely absorbed in the calorimeter, it is possible to make differential measurements of 
the various types of radiation emitted by a radioactive source by varying the thickness of the container for the source. For sources of the magnitude previously available relatively sensitive calorimeters were required. For example, one curie of polonium develops heat at the approximate rate of $25 \mathrm{cal} / \mathrm{hr}$. To measure the heat from a source containing a few millicuries with fair accuracy, a microcalorimeter of a capacity of the order of 1 cal is required. Even then, with highest degree of thermal insulation the temperature will rise by only a fraction of a degree per hour. With large sources measurements can be made with less sensitive devices.

An example of a microcalorimeter as described by Orthman [8] is shown in figure 17. The calorimetric vessels consist of two copper cylinders, $12 \mathrm{~mm}$ in diameter and $12 \mathrm{~mm}$ high, as nearly identical as possible. One is shown in section at figure 17 , a. A cavity, $H, 4 \mathrm{~mm}$ in diameter and $4 \mathrm{~mm}$ high is provided in each vessel. In this cavity of one of the copper cylinders the radioactive preparation is placed. The extra copper cylinder is used to provide the reference temperature to determine the heat developed in the first. In the annular channel $C$, there are 15 thermoelements imbedded in Wood's metal. In an outer, deeper channel. $D$, is a heating coil through which a measured electric current can be passed to calibrate the calorimeter. In use a pair of these copper cylinders is suspended in an evacuated flask, $F$, mounted in a copper box, $B$, which is suspended in a Dewar bulb containing water at a constant temperature. Devices of this type can measure the generation of heat at the rate of $5 \times 10^{-3}$ $\mathrm{cal} / \mathrm{hr}$ with an over-all accuracy of 2 or 3 percent. Measurement of the energy released by the beta-ray disintegration of $\mathrm{RaE}$ by calorimetric methods played an important role in the development of the theory of the neutrino to explain the nature of continuous beta-ray spectra. The measurements of the heat developed by the absorption of the beta rays agreed quite accurately with that computed from the observed beta ray spectrum, showing that nuclear disintegration beta rays are emitted in a continuous spectrum.

\section{MEASUREMENT OF IONIZATTION CURRENTS}

The measurement of ionization currents produced by radioactive radiations requires the use of electrical instruments with the best degree of electrical insulation and a high sensitivity. Since $i=\triangle Q / \triangle t=C \triangle V / \triangle t$, where $i$ is the ionization current, $Q$ the electrical charge, and $V$ the resulting voltage, it is important that $C$, the electrical capacitance, be small. The principal devices for this purpose are electroscopes, electrometers, and vacuum-tube electrometers. Whereas electroscopes may be constructed as a part of the ionization chamber, or even be mounted within it, electrometers and electrometer tubes require a separate chamber.

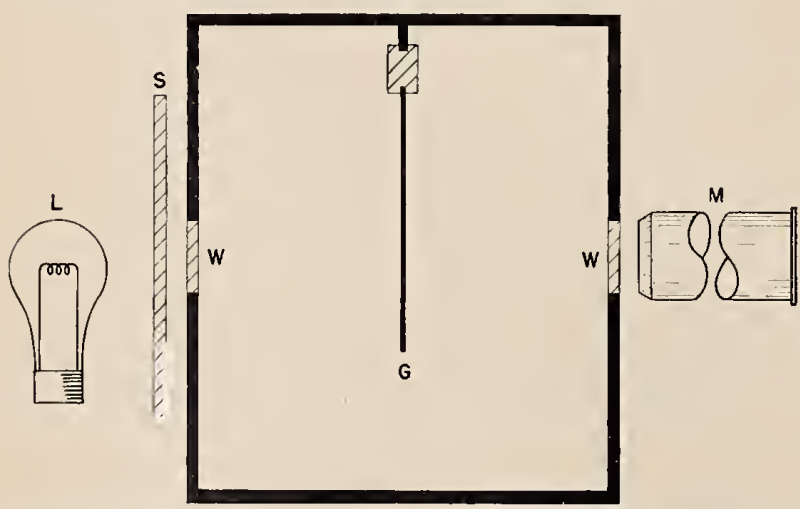

FIGURE 18. Gold-leaf electroscope; $M=$ microscope; $W W=w i n d o w s ; G=$ gold leaf; $S=$ diffusing screen; $L=\operatorname{lamp}$.

\section{Electroscopes}

The simplest of the electroscopes is the goldleaf electroscope shown diagrammatically in figure 18.

When the insulated leaf support is charged to a potential of several hundred volts, the leaf is repelled to an angle of 40 or 50 degrees from the support and will remain in this position if the insulation is perfect and there are no ions in the gas. Since neither condition can be fulfilled completely, the gold leaf will fall slowly to the uncharged position. The rate of motion of the gold leaf is observed by a lowpower microscope with a graduated scale in the eyepiece. The background or natural rate of fall, of the gold leaf is determined and subtracted from the rate observed with a source of radiation. Since the motion of the gold leaf is rarely uniform over all divisions of the scale, the motion is always observed between the same two selected divisions of the scale. Sources of widely different intensities cannot be compared accurately with the gold leaf electroscope, as at high rates of drift the rate is no longer proportional to the ionization.

Gold leaf electroscopes can be used to measure alpha rays by introducing the source into the electroscope chamber, as shown at $A$ in figure 19. This form of the electroscope has a leaf system of very low capacity and therefore 


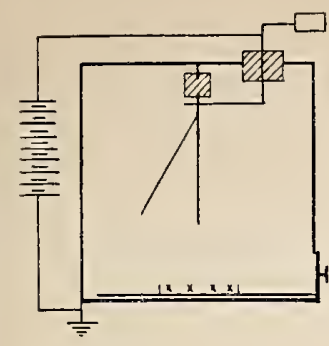

a
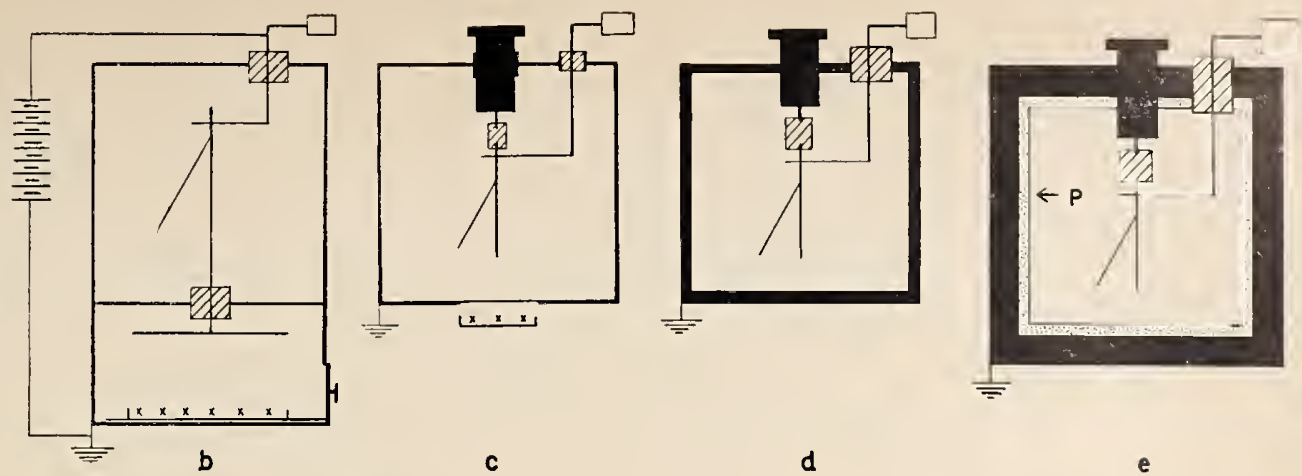

FIGURE 19. Various types of gold-leaf electroscopes.

$\mathrm{a}=$ Alpha-ray electroscope of low electrostatic capacity; $\mathrm{b}=$ alpha-ray electroscope with separate ionization cbamber (high electrostatic capa city); $c=$ beta-ray electroscope; $d=$ gamma-ray electroscope with thick wall; $e=$ neutron electroscope; $p=1 a y e r s$ of paraffin. Heavy lead walls reduce effect of gamma rays.

of high sensitivity. Where lower sensitivity is satisfactory, the modification shown in figure 19 , B may be used, where the ionization is produced in a separate chamber into which the source is inserted.

Beta rays may be measured by providing a thin window through which the beta rays may enter the electroscope chamber, as shown in figure 19, C. When comparing similar sources having beta rays of the same energies, corrections for absorption in the window may be neglected.

A gamma ray electroscope shown in figure $19, \mathrm{D}$ is surrounded by sufficient lead to exclude all beta rays. In the case of complex sources of radiation, as represented by a sealed preparation of radium, this lead is made thick enough to exclude also the softer gamma rays.

To measure neutrons with an electroscope, the effect of gamma rays that may also be emitted by the source must be reduced by a heavy shield of lead. The intensity of the neutrons will be reduced slightly in passing through the lead. Since the neutrons will not themselves produce ionization of the air in the electroscope, a lining of paraffin is used, (shown at $P$ in figure 19, E) from which the neutrons expel protons. The protons in turn produce ionization proportional to the intensity of the incident neutrons.

\section{Quartz Fiber Electroscopes}

Lauritsen [9] has described a quartz fiber electroscope that has many advantages over the type with gold leaf. It is more compact, less sensitive to levelling, and is more readily portable. As a result of its lower electrical capacity, it is relatively more sensitive. The sensitive element consists of a fine metallized quartz fiber, mounted on a parallel metal support as indicated in figure 20. The metal support is mounted on a small insulator and may be charged from a battery or other source of potential by pressing the charging key. A small piece of quartz fiber mounted across the end

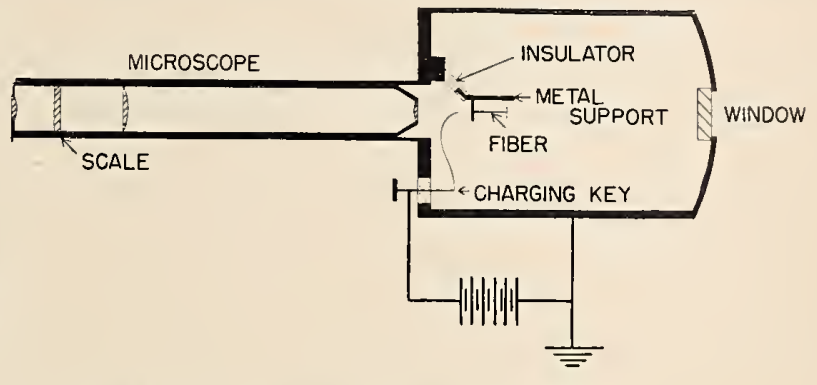

FIGURE 20. Lauritsen electroscope.

of the metallized fiber serves as an index that is viewed through a microscope. with eyepiece scale. On being charged the metallized fiber is deflected from the support and returns toward the position of zero charge when the gas in the chamber is ionized. A window is provided for illuminating the fiber. Approximately $200 \mathrm{v}$ are required to produce a full-scale deflection of the fiber. The background rate is of the order of 4 divisions per hour and the sensitivity is about 2 divisions per minute for $1 \mathrm{mg}$ of radium at 1 meter.

\section{Electrometers}

These devices may be differentiated from electroscopes in that they require steady auxiliary potentials to produce electric fields in which an indicating system, sometimes called the "needle," moves under the influence of potentials to be measured. Several vacuum tubes may also be classed with electrometers, since they may be used at various degrees of sensitivity depending on the value of the auxiliary potentials, mechanical adjustments, and external electrical capacity connected to them.

The usual principles of operation may be illustrated by reference to the Dolezalek quadrant electrometer, although this instrument is no longer in general use since it has been superseded by more compact instruments less sensitive to vibrations and levelling and less easily damaged by accidental overloading. The Dole- 
This method may also be converted to a null deflection method by connecting a small calibrated auxiliary resistor between the lower end of $R$ and ground and developing a potential across it equal and opposite to that developed

TO IONIZATION CHAMBER

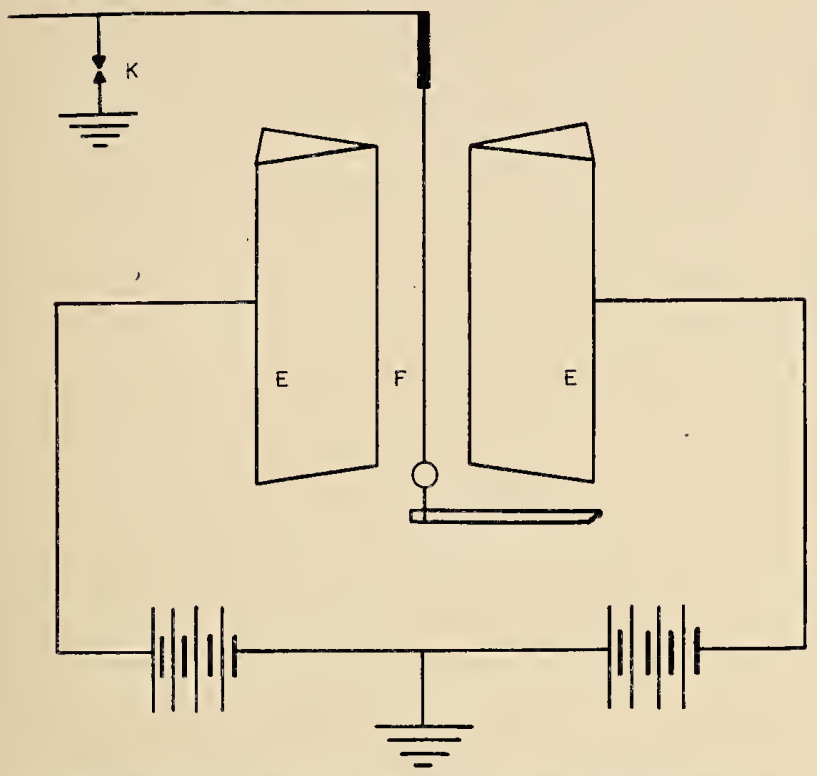

FIGURE 24. Schematic diagram of string electrometer. $E, E=$ knife edges; $F=$ quartz fiber; $K=$ grounding key.

in $R$ by the ionization current. This potential may be determined by measuring the current through the auxiliary resistor. When the electrometer reading is zero, $i R=I r$, where $r$ is the value of the auxiliary resistance and $I$ the current through it. It is assumed that the value of $r$ may be neglected in comparison with $R$, which is usually the case.

\section{String Electrometers}

Numerous types of string electrometers have been developed, but one of the most satisfactory is due to Lutz in which a fine metal, or metallized quartz fiber is stretched by a fine quartz spring between two triangular metal knife edges. These knife edges are maintained at steady equal positive and negative potentials. The voltage to be measured is applied to the fiber. This causes it to move from the uncharged position to a new position of equilibrium in the plane of the knife edges. A diagram of the instrument is shown in figure 24 . It has a relatively low electrical capacity, is only moderately sensitive to vibration and levelling, and is adaptable to automatic photographic recording of the position of the fiber. The insulators for the fiber system must be of highest quality. It shares with most electrometers the difficulty of securing and maintaining a uniformly high degree of insulation of the moving system. The sensitivity is adjusted by altering the separation of the knife edges or changing the poten-

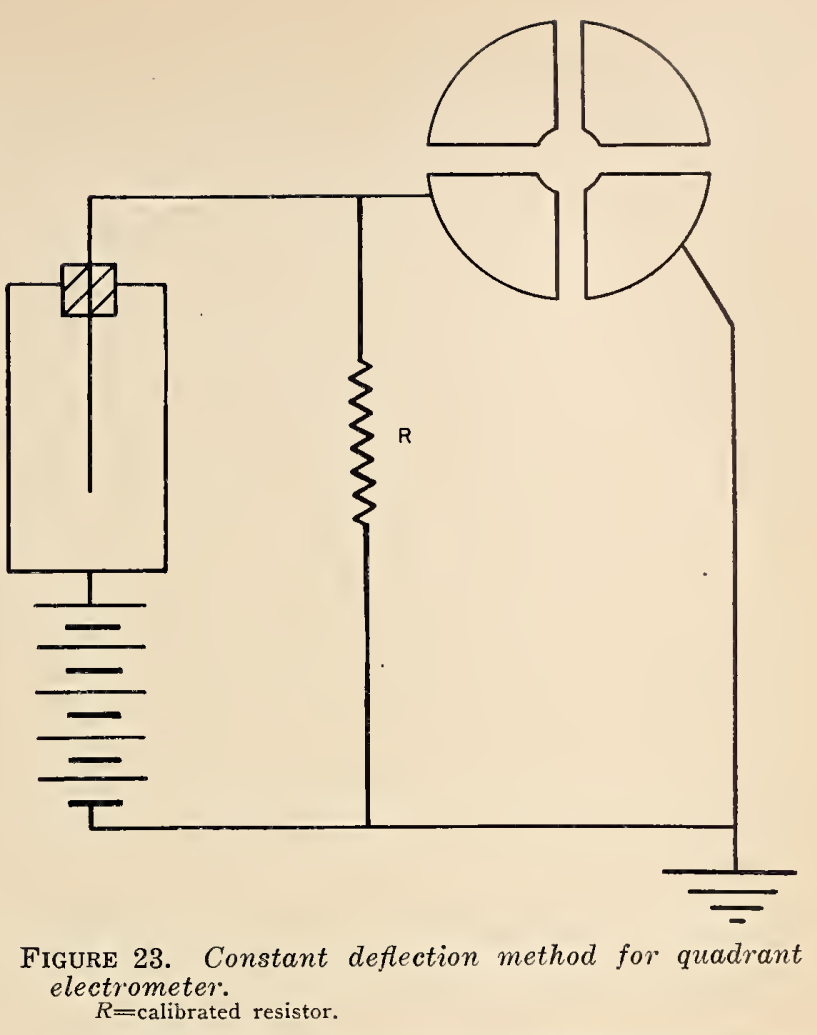

tial applied to them and by altering the tension of the fiber.

\section{Portable Electrometers}

Lindemann has devised a portable electrometer of compact arrangement that is based on the quadrant principle. However, since the needle is a metallized quartz fiber mounted on a taut metallized quartz suspension, this instrument is not unduly sensitive to vibration and can be operated in any position. The electric field in which the needle moves is provided by applying opposite potentials to each pair of four metal plates mounted symmetrically about the needle. It has a sensitivity of about 500 divisions per volt and an electrical capacity of the order of 2 esu.

In a further modification by Dershem the sensitivity of the taut fiber electrometer has been increased to several times that of the Lindemann electrometer. Part of this increase is obtained by mounting a mirror at the suspension point of the needle and using optical magnification of the motion. The needle in this instrument is free to rotate completely about its mounting without touching the quadrants. This protects it from damage resulting from short circuiting the quadrant voltage. Recent improvements in methods for producing uniform quartz fibers for use in the Lindemanntype electrometers have resulted in the development of instruments superior to any previously 
available. The compactness, relative ruggedness, and stability of these instruments, combined with their high sensitivity makes them suitable for most applications where an electrometer can be used.

\section{Electrometer Tubes}

The development of special vacuum tubes with low grid currents and high insulation of the control grid by a space-charge electrode has made possible the amplification of direct currents. Improvements in the associated circuits have resulted in amplifiers that extend the range of these tubes to the smallest currents conveniently measured by electrometers.

In the most sensitive circuits a single $12-\mathrm{v}$ storage battery provides the current for operating the electrometer tube. A network of resistors in series with the filament is arranged to provide voltages for the plate, the space charge grid, and the control grid. The connections are arranged to compensate for slow changes in the electromotive force of the battery and for changes in the filament emission from other causes. The compensation for changes in the battery is accomplished by obtaining the plate, space charge grid, and control grid voltages from resistors in series with the filament. Additional compensation for changes of filament emission independent of the heating current is obtained by balancing changes in the plate current against changes in the space charge grid current. A typical example of a stabilized circuit developed by $\mathrm{Du}$ Bridge and Brown [11] is shown in figure 25.

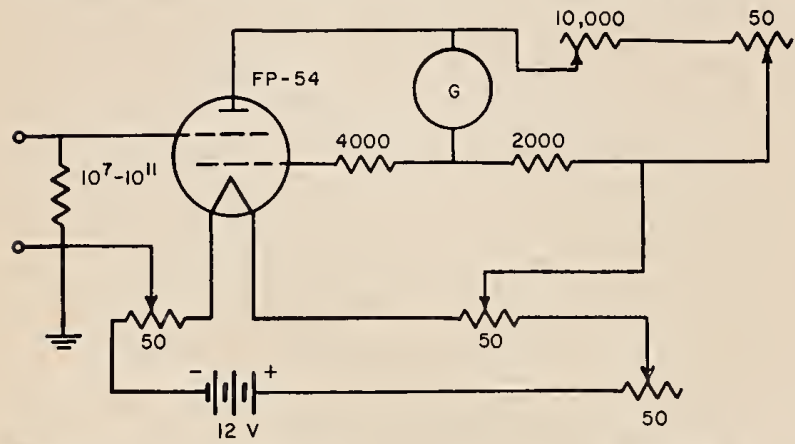

FIgURE 25. DuBridge-Brown circuit for FP-54 electrometer tube.

The sensitivity of such circuits is quite high, particularly when the tube and control grid connections are mounted in an evacuated chamber to reduce the effect of stray ionization. Currents of the order of $10^{-14}$ amp can be measured readily, and this limit can be extended several orders of magnitude under special conditions. A convenient form of circuit operated from the alternating-current mains has been developed by Anderson, Lawson, and Weil [12], and is shown in figure 26 . It is to be noted that each stage has a separate heater winding to avoid heater cathode leakage. It is important that the power transformer give sufficient volt-

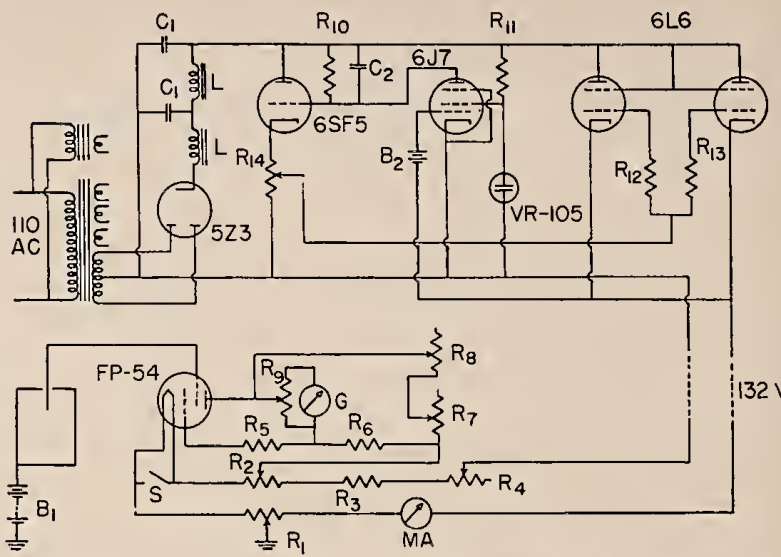

Figure 26. Circuit for operating FP-54 tube from alternating current mains, eliminating storage batteries.

$C_{1}, C_{1}=8 \mathrm{mfd} ; L_{1}, L_{1}=30$ henry, $110 \mathrm{ma} ; C_{2}=0.05 \mathrm{mfd} ; B_{1}=$ 135 volt battery; $R_{1}=400$ ohms; $R_{2}=50$ ohms; $R_{3}=1,400$ ohms $R_{4}=50$ ohms: $R_{5}=4,000$ ohms; $R_{B}=2,000$ ohms; $R_{7}=50$ ohms: $R_{\mathrm{s}}=10,000$ ohms: $R_{9}=$ Ayrton shunt : $R_{10}=100000$ ohms: $R_{11}=$ 15,000 ohms; $R_{12}=200,000$ ohms; $R_{13}=100,00$ ohms; $B_{2}=135$ volt battery.

age as well as current in the high-voltage winding to allow for the loss of voltage in the regulator circuit.

Recently a split FP-54 tube has been developed [13], which compensates for variations in the emission from the filament by placing the pairs of control grids and plates in a bridge circuit. A single filament and space charge grid are used. The wiring diagram suggested for this split FP-54 is shown in figure 27 . With a circuit of a sensitivity of $50,000 \mathrm{~mm} / \mathrm{v}$, using a grid resistor of $10^{10} \mathrm{ohms}$, the long-time drift is less than $10 \mathrm{~cm}$ per week and the short-time fluctuations are about three times the thermal noise of the grid resistor, computed for a bank width of one cycle per second.

\section{Vibrating Reed Electrometer}

In 1932 A. W. Hull [14] suggested the use of a vibrating reed for modulating a small direct voltage, and the idea was developed into a practical model by LeCaine and Waghorne [15]. The device, which is a modification of the generating voltmeter, is shown schematically in figure 28. The vibrating reed, $R$, is fixed in an insulating mounting. The dimensions of the reed are chosen so that its natural frequency of vibration coincides with the frequency of alternating current supplied to the electromagnet, $N$, which maintains the steady oscillation of the reed.

The diagram shows connections for measuring an ionization current in the chamber, $I$, which develops a potential on the fixed electrode, $E$, of the modulating condenser as it 


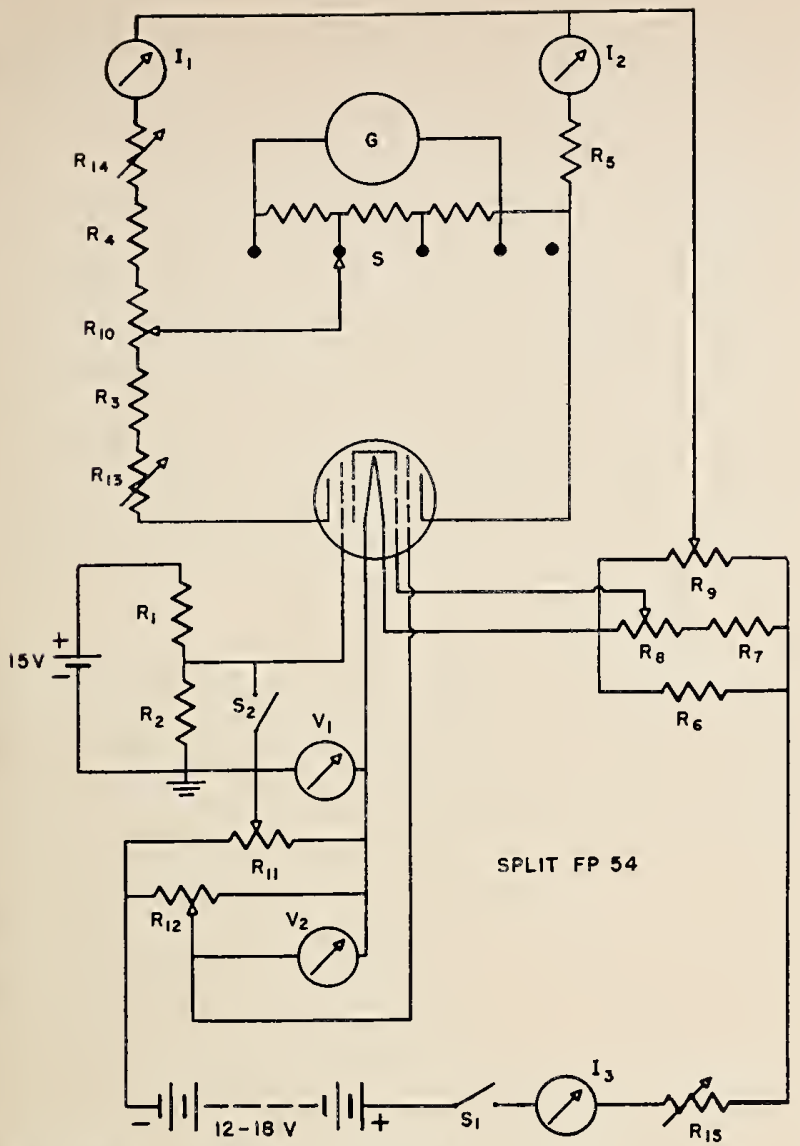

FIgure 27. Circuit for split FP-54 tube.

$R_{1}=150,000$ ohms; $R_{2}=1,000$ ohms; $R_{3}=0$ to 20,000 ohms; $R_{5}=0$ to 20,000 ohms; $R_{5}=50,000$ ohms; $R_{6}=100$ ohms; $R_{7}=$ 75 ohms; $R_{\mathrm{s}}=125$ ohms; $R_{9}=200$ ohms; $R_{10}=5,000$ ohms; $R_{11}=125$ ohms; $R_{12}=125$ ohms; $R_{13}=400$ ohms; $R_{14}=50,000$ ohms: $R_{15}=35$ ohms; $S=$ Ayrton shunt; $S_{1}, S_{2}=$ knife switches; $V_{1}, V_{2}=0$ to 10 volt voltmeter; $I_{1}, I_{2}=0$ to 100 microamperes meters; $I_{3}=0$ to 100 milliamperemeter; $C=$ galvanometer; sensitivity $2 \times 10^{-10} \mathrm{amp} / \mathrm{mm}$.

flows through the resistor, $r$. The reed is connected to the input of the resistance capacity coupled amplifier, which amplifies the modulated voltage. The amplitude of this modulated

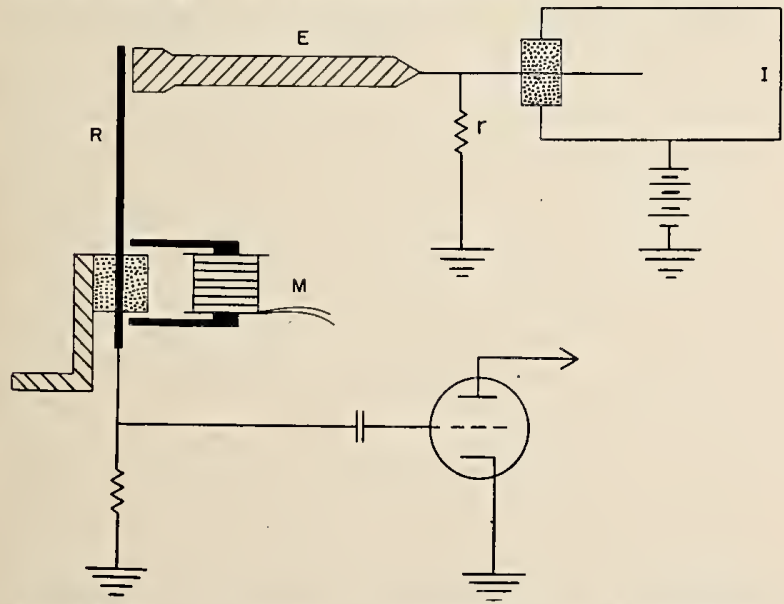

FIGURE 28. Diagram of vibrating reed electrometer. $R=$ vibrating reed operated by electromagnet, $M ; E=$ stationary electrode; $\mathrm{r}=$ calibrated resistor; $I=$ ionization chamber. voltage is proportional to the steady voltage on $E$. A very low noise level may be obtained by such an arrangement since the output of the amplifier may be filtered to exclude all frequencies except that of the reed. The amplified modulated voltage may be rectified, and a direct-current meter of moderate sensitivity may then be used as an indicator.

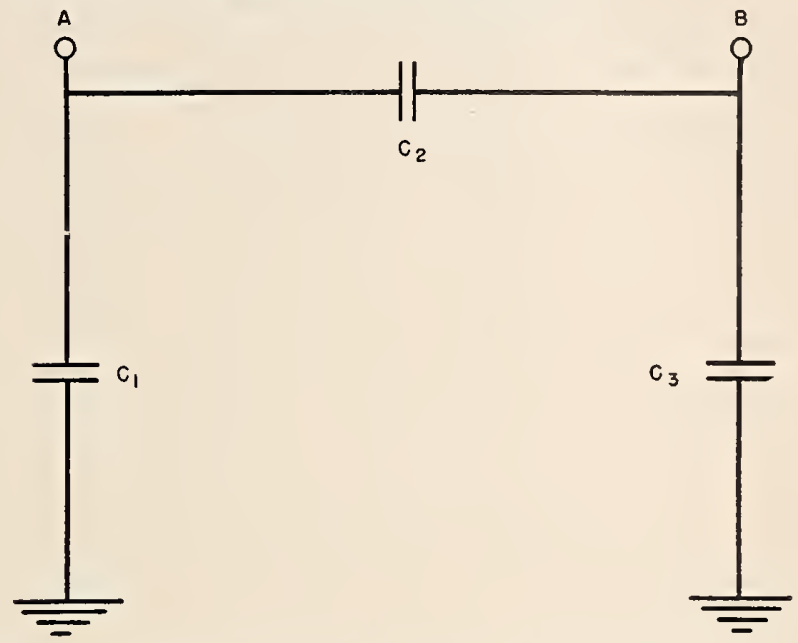

FIGURE 29. Schematic circuit of the generating voltmeter.

$C_{1}, C_{2}=$ fixed capacitors; $C_{3}=$ variable capacitor.

The principle of the generating voltmeter, on which the vibrating reed electrometer is based, may be illustrated by the diagram in figure 29. If $C_{1}$ and $C_{3}$ are fixed, and the capacity of $C_{2}$ is a periodic function of the time, the alternating voltage at $B$ is proportioned to the voltage at $A$ and may be used to measure their voltage.

\section{Ionization Chambers}

Electrometers require separate ionization chambers that may vary considerably with the type of radiation to be measured. The general requirements common to all types are: adequate insulation for the collecting electrode; protection of the insulation from high electric fields; adequate voltage between the electrodes to insure a reasonable degree of saturation; and a small capacity for the collecting electrode. A typical arrangement for the central electrode and guard ring is shown in figure 30 . It is also important that the inner surface of the chamber be as free as possible from radioactive contamination. Copper, steel, and brass surfaces when freshly machined and kept from contact with sources of contamination will give about 6 to 9 alpha particles per hour per $100 \mathrm{~cm}^{2}$. Without this treatment the number may be many times this figure. Special grades of sandpaper, known to be free of radioactive contamination, may also be used to clean the interior surfaces. Lead and solder are frequently con- 


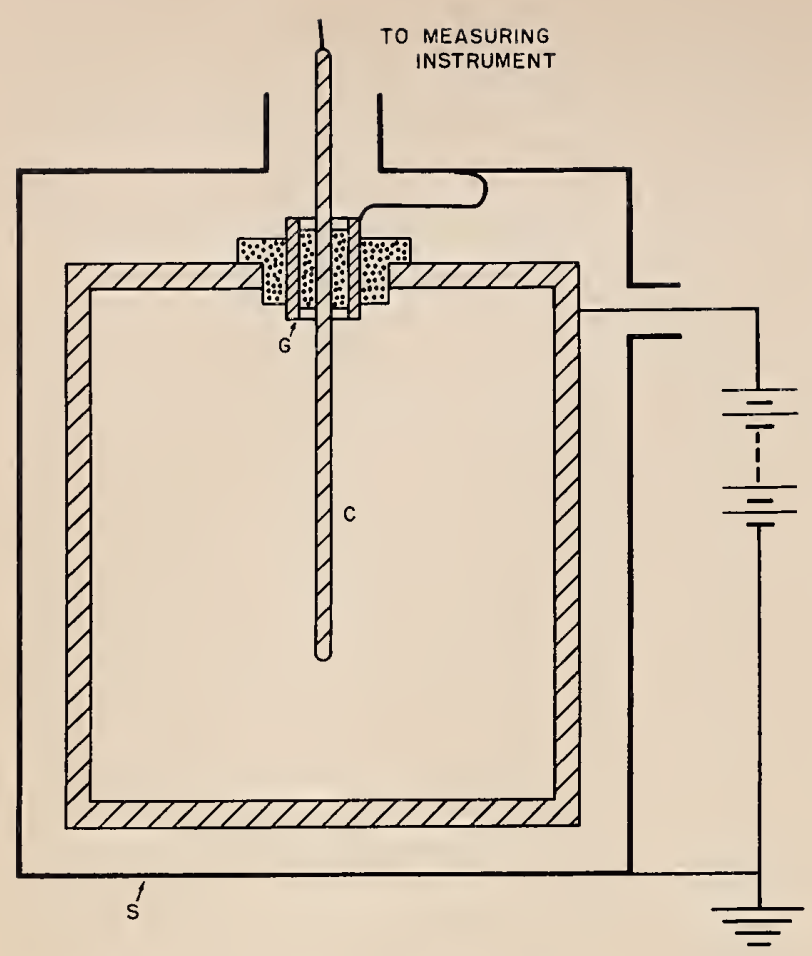

FIgURE 30. Cross section of typical ionization chamber. $G=$ guard ring; $C=$ ion-collecting electrode; $S=$ shield. taminated. Therefore, no lead solder should be exposed to the interior. Reduction of the background is important when small ionization currents are to be measured. The insulator supporting the collecting electrodes should be protected by a guard ring, insulated from the chamber to which the collecting potential is applied, and connected to earth. Gamma-ray chambers should be surrounded by approximately $0.5 \mathrm{~cm}$ of lead to exclude beta rays, and beta-ray chambers must be provided with a window thin enough to admit a large proportion of the radiation. Ionization chambers for measurement of neutrons may be coated on the inside with boron, or a thin layer of paraffin covered with a thin conducting layer, and must be heavily shielded with lead when gamma rays are also present.

Although air is a satisfactory gas for use in many ionization chambers, argon and heavy vapor, such as methyl and ethyl bromide, are more effective when most of the ionization is produced in the gas itself, as in the case of soft gamma rays and $\mathrm{X}$-rays.

\section{Radioactive Substances}

Distinction is still made between naturally occurring radioactive isotopes and radioactivity produced by subjecting naturally stable elements to nuclear reactions by which they become radioactive artificially. However, it is now recognized that in principle the two are indistinguishable.

\section{Natural Radioactivity}

Elements of high atomic number are generally unstable in nature. The majority of the known isotopes of atomic number 81 or higher are naturally radioactive. This results in the formation of relatively long chains or families in which each successive isotope transforms into a new radioactive isotope, until finally a stable form is reached. Among these elements transformation by the emission of an alpha particle is quite common, whereas among artificially produced isotopes of lower atomic number this mode of disintegration is infrequent.

Careful physical and chemical investigation extending over a period of several decades has led to the identification of three families, which include nearly all naturally radioactive isotopes accounting for all of the naturally occurring elements of high atomic numbers that are known at present. These families are called the uranium, thorium, and actinium families from the parent isotopes from which they are presumed to originate. These groups are distinctly separate in that there are no known nuclides common to any of the three. The uranium family starts with $\mathrm{U}^{238}$, the thorium family with $\mathrm{Th}^{232}$, and the actinium family has been traced back to $\mathrm{U}^{235}$, which, although an isotope of $\mathrm{U}^{238}$, is not genetically related to it.

There are various methods for representing these families in a chart to show the successive isotopes in each chain. Figure 31 represents one form in which the ordinates represent the number of neutrons present in the nucleus in excess of the number of protons. The abscissa represents the atomic number, $Z$, equal to the number of protons in the respective nuclei. The mass numbers are therefore obtained from the sum $2 Z+N$. Alpha disintegrations are represented by a horizontal line from right to left, corresponding to a decrease of nuclear charge by 2 units and of mass by 4 units. Beta disintegrations are represented by a diagonal line from left to right representing an increase of one unit of nuclear charge with no change in mass number.

In table 2 are given the half-periods, decay constants, ranges of alpha particles, and approximate energies of beta and gamma rays. 


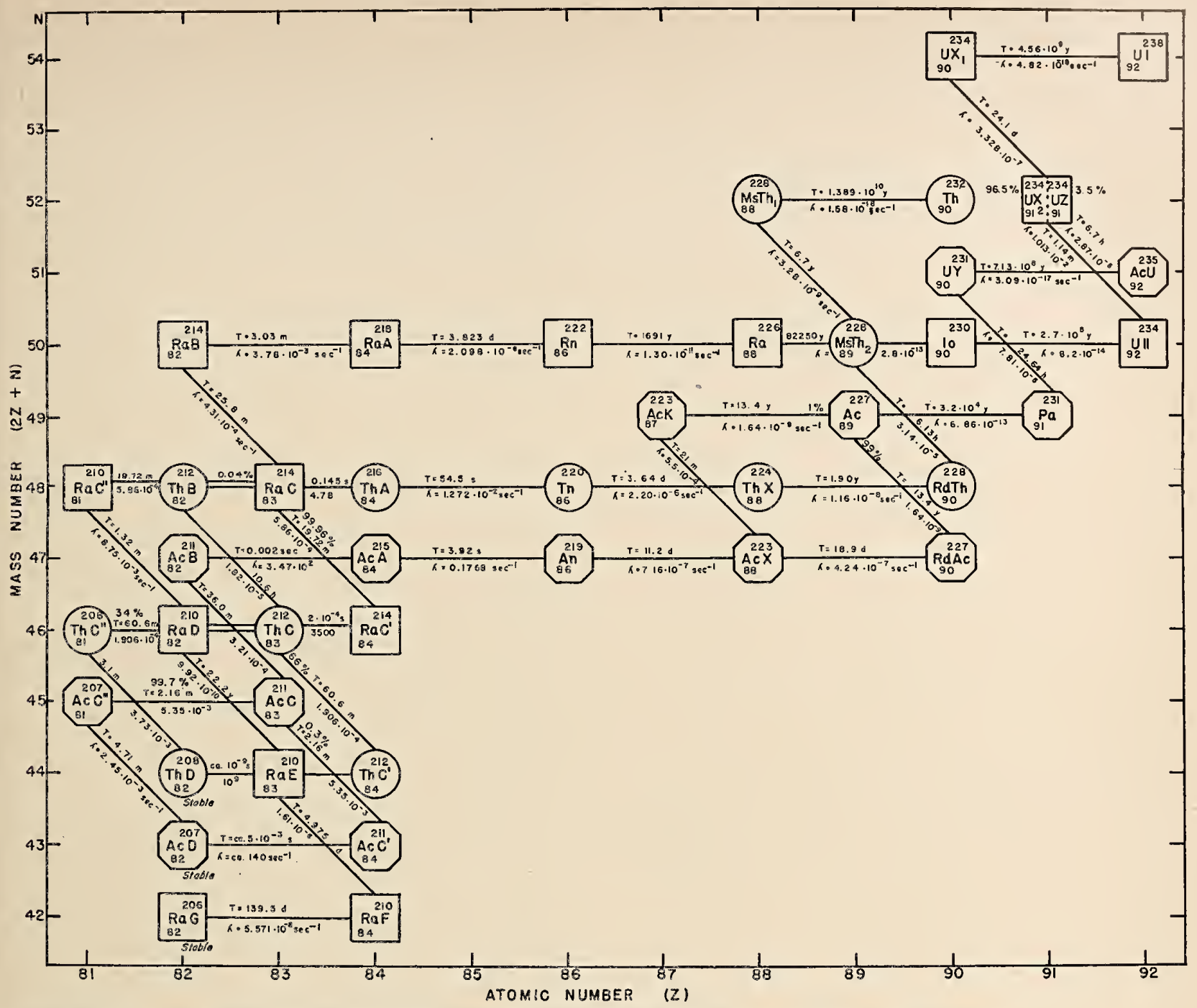

FIGURE 31. Chart showing genetic relationship of the uranium, thorium, and actinium families. Abscissa are atomic numbers, ordinates $=M-2 Z$, where $M$ is the mass number.

The practical usefulness of naturally radioactive isotopes depends on the half-life, the nature and energy of the radiations emitted as well as an abundance in nature and ease of chemical separation. Those emitting strong gamma radiation are particularly useful. A preparation of such isotopes may be sealed in a glass or metal container and used with very little loss of radiation by adsorption in the walls. Gamma radiation has found considerable use in medical treatments of malignancies and in the radiography of thick metals. In the uranium family, radium has been found to fill the requirement of a suitable half-life, and the isotopes in equilibrium with it provide a strong source of gamma radiation. A preparation of radium is about $2.8 \times 10^{6}$ times as active as an equal weight of uranium, since the half-periods have this ratio. Furthermore, the half-period of radium, 1630 years, is still sufficiently long to provide a practically constant source. Where gamma-ray preparations of small volume are required for short periods, radon is more satisfactory than radium. The radon in equilibrium with $1 \mathrm{~g}$ of radium has a volume of only 0.65 $\mathrm{mm}^{3}$, and yet has all the products of the radium family in equilibrium with it that emit strong gamma radiation.

From 5 to 50 tons of uranium ore are required to produce $\mathbf{1} \mathbf{g}$ of radium. The elaborate chemical operations required account for the relatively high cost of radium. Radon is released freely into the water of a solution of radium. The radon is separated by pumping off the gases and vapors from an enclosed solution of radium, the equilibrium amount being available every 30 days.

Radium is also used commercially in the preparation of a self-luminous compound consisting of a mixture of radium and zinc sul- 
TABLE 2.

\begin{tabular}{|c|c|c|c|c|c|}
\hline & $\lambda \sec ^{-1}$ & $T$ & $\begin{array}{l}a \\
\text { mean } \\
\text { range }\end{array}$ & $\beta$ rays Mev & $\gamma$ rays Mev \\
\hline UI & $4.82 \times 10^{-18}$ & $4.5 \times 10^{9} \mathrm{y}$ & $\begin{array}{l}m m \\
2.69\end{array}$ & Mev & Mev \\
\hline UXi & $3.33 \times 10^{-7}$ & $24.1 \mathrm{~d}$ & $\ldots$ & $0.11 ; 0.30$ & 0.092 \\
\hline $\mathrm{UX}_{2}$ & $1.01 \times 10^{-2}$ & $1.14 \mathrm{~m}$ & $\ldots$ & $2.32 ; 1.5$ & .8 \\
\hline UZ & $\mid$\begin{tabular}{|l|}
$2.87 \times 10^{-5}$ \\
$8.2 \times 10^{-14}$
\end{tabular} & $\begin{array}{l}6.7 \mathrm{~h} \\
2.7 \times 10^{5} \mathrm{y}\end{array}$ & $\ddot{3} \ddot{24}$ & $0.56 ; 1.55$ & $\begin{array}{l}.7 \\
. .\end{array}$ \\
\hline Io & $2.8 \times 10^{-13}$ & $8.23 \times 10^{\frac{4}{4}} \mathrm{y}$ & 3.15 & $\cdots$ & $\because$ \\
\hline $\begin{array}{l}\mathrm{Ra} \\
\mathrm{Rn}\end{array}$ & $1.30 \times 10^{-11}$ & $1630 \mathrm{y}$ & $\begin{array}{l}3.29 \\
4.04\end{array}$ & $\cdots$ & .19 \\
\hline RaA & $3.78 \times 10^{-3}$ & $3.05 \mathrm{~m}$ & 4.64 & 0.04 & $\cdots$ \\
\hline $\operatorname{RaB}$ & $4.31 \times 10^{-1}$ & $25.8 \mathrm{~m}$ & $\ldots$ & 0.65 & $\begin{array}{ll}0.24: & 0.3 ; \\
0.35 ; & 0.466\end{array}$ \\
\hline $\mathrm{RaC}$ & $5.86 \times 10^{-4}$ & $19.7 \mathrm{~m}$ & 4.06 & 3.15 & $\begin{array}{l}0.426 ; 0.51 ; \\
0.61 ; 0.77 ; \\
9.94 ; 1.13 ; \\
1.24 ; 1.39 \\
1.78 ; 2.22\end{array}$ \\
\hline $\mathrm{RaC}^{\prime}$ & 4660 & $1.48 \times 10^{-1} \mathrm{sec}$ & 6.87 & & \\
\hline RaN & $\mid \begin{array}{l}8.75 \times 10^{-3} \\
9.92 \times 10^{-10}\end{array}$ & $\begin{array}{l}1.32 \mathrm{~m} \\
22.2 \mathrm{y}\end{array}$ & $\ldots$ & $\begin{array}{l}1.8 \\
0.025\end{array}$ & $\cdots 0.047$ \\
\hline $\mathrm{RaE}$ & $1.61 \times 10^{-3}$ & $4.98 \mathrm{~d}$ & $\cdots$ & 1.17 & ......... \\
\hline $\begin{array}{l}\mathrm{RaF} \\
\mathrm{AcU}\end{array}$ & $\begin{array}{l}5.5 \times 10^{-6} \\
2.59 \times 10^{-17}\end{array}$ & $\begin{array}{l}139.5 \mathrm{~d} \\
8.52 \mathrm{x} 10^{8} \mathrm{y}\end{array}$ & $\begin{array}{l}3.83 \\
2.85\end{array}$ & & \\
\hline $\begin{array}{l}\mathrm{UY} \\
\mathrm{Pa}\end{array}$ & $\begin{array}{l}7.81 \times 10^{-5} \\
6.86 \times 10^{-13}\end{array}$ & $\begin{array}{l}24.6 \mathrm{~h} \\
3.2 \times 10^{4} \mathrm{y}\end{array}$ & 3.63 & $\sim 0.2$ & $0.095 ; 0.29$ \\
\hline $\begin{array}{l}\mathrm{Ac} \\
\mathrm{AcK} \\
\mathrm{RdAC}\end{array}$ & $\begin{array}{l}1.64 \times 10^{-9} \\
5.5 \times 10^{-1} \\
4.24 \times 10^{-1}\end{array}$ & $\begin{array}{r}13.4 \mathrm{y} \\
21 \mathrm{~m} \\
18.9 \mathrm{~d}\end{array}$ & $\begin{array}{l}3.5(1 \%) \\
3.66 \\
4.34\end{array}$ & $\begin{array}{l}0.22 \\
1.20\end{array}$ & $\begin{array}{l}\cdots 3 \\
0.15 \\
0.25 \\
0.30\end{array}$ \\
\hline AcX & $7.16 \times 10^{-\pi}$ & $11.2 \mathrm{~d}$ & 4.31 & & $\begin{array}{l}0.143 ; 0.15 \\
0.20 ; 0.27\end{array}$ \\
\hline $\begin{array}{l}\mathrm{An} \\
\mathrm{AcA}\end{array}$ & $\begin{array}{l}0.177 \\
3780\end{array}$ & $\begin{array}{l}3.92 \mathrm{~s} \\
1.83 \times 10-\mathrm{s}\end{array}$ & $\begin{array}{l}5.68 \\
6.44\end{array}$ & & \\
\hline $\begin{array}{l}\mathrm{AcB} \\
\mathrm{AcC}\end{array}$ & $\begin{array}{l}3.21 \times 10^{-1} \\
5.35 \times 10^{-3}\end{array}$ & $\begin{array}{l}36.0 \mathrm{~m} \\
2.16 \mathrm{~m}\end{array}$ & & $0.5 ; 1.4$ & $\begin{array}{c}0.8 \\
0.35 ; 0.46\end{array}$ \\
\hline $\mathrm{AcC}^{\prime}$ & $\sim 140$ & $-5 \times 10^{-3} \mathrm{~s}$ & 6.53 & & 0.48 \\
\hline $\begin{array}{l}\mathrm{AcC}{ }^{\prime \prime} \\
\mathrm{Th}\end{array}$ & $\begin{array}{l}2.45 \times 10^{-3} \\
1.58 \times 10^{-18}\end{array}$ & $\begin{array}{l}4.71 \mathrm{~m} \\
1.39 \times 10^{10} \mathrm{y}\end{array}$ & $2 \ddot{84}$ & 1.47 & \\
\hline $\begin{array}{l}\text { MsTh } \\
\text { MsTh }\end{array}$ & $\begin{array}{l}3.28 \times 10^{-9} \\
3.14 \times 10^{-4}\end{array}$ & $\begin{array}{l}6.7 \mathrm{y} \\
6.13 \mathrm{~h}\end{array}$ & $\ldots$ & $\begin{array}{l}0.053 \\
1.55\end{array}$ & $\begin{array}{l}0.058 ; 0.079 \\
0.129 ; 0.184 ; \\
0.249 ; 0.319 ; \\
0.338 ; 0.408 ; \\
0.462 ; 0.915 \\
0.970\end{array}$ \\
\hline $\begin{array}{l}\text { RdTh } \\
\text { ThX }\end{array}$ & $\begin{array}{l}1.16 \times 10^{-8} \\
2.20 \times 10^{-1}\end{array}$ & $\begin{array}{l}1.90 \mathrm{y} \\
3.64 \mathrm{~d}\end{array}$ & $\begin{array}{l}3.96 \\
4.26\end{array}$ & & $\begin{array}{c}0.088 \\
\ldots \ldots \ldots\end{array}$ \\
\hline $\begin{array}{l}\text { Tn } \\
\text { ThA } \\
\text { ThB }\end{array}$ & $\begin{array}{l}1.27 \times 10^{-2} \\
414 \\
1.82 \times 10^{-5}\end{array}$ & $\begin{array}{l}54.5 \mathrm{~s} \\
0.158 \mathrm{~s} \\
10.6 \mathrm{~h}\end{array}$ & $\begin{array}{l}5.0 \\
5.62 \\
0.36\end{array}$ & & $\ldots \ldots \ldots$ \\
\hline \multirow{2}{*}{$\begin{array}{l}\text { ThC } \\
\text { ThC } \\
\text { The" }\end{array}$} & $1.906 \times 10^{-1}$ & $60.6 \mathrm{~m}$ & 4.71 & 2.2 & 0.30 \\
\hline & $\widetilde{3.73 \times 10^{-3}}$ & $\begin{array}{l}3 \times 10^{-i} \mathrm{~s} \\
3.1 \mathrm{~m}\end{array}$ & 8.53 & 1.82 & $\begin{array}{l}0.29 ; 0.51 \\
0.59 ; 0.73 \\
0.78-2.65\end{array}$ \\
\hline
\end{tabular}

phide. The energetic alpha particles from the source of the disintegration products of radium produce fluorescence of the zinc sulphide crystals. This mixture is applied to dials of clocks and instruments; particularly those that are used where other methods of illumination would be objectionable. The greatest use is for military equipment. During World War II several hundred grams of radium were used for this purpose.

Radium is measured in milligrams of the element. In practice, quantities of radium are determined by comparing the gama radiation from an unknown sample with that from a carefully weighed standard, by using electroscopes or ionization chambers. When a range of standards is available to permit one to be selected near the value of the unknown, this gives a reliable determination. When the un- known is in a container with walls differing from that of the standard, a correction must be made for the absorption of the gamma radiation in the walls of the container to obtain the amount of radium. For platinum containers commonly used in medical treatment this correction may be as high as 12 percent, and depends on the characteristics of the device used to measure the ionization current produced by the gamma radiation as well as on the geometrical relations between the source and the instrument.

Radon is measured in curies, a curie being defined officially as that quantity of radon in equilibrium with $1 \mathrm{~g}$ of radium. Thus, 1 curie of radon has a gamma radiation equivalent to $1 \mathrm{~g}$ of radium.

The curie has been extended officially by the International Radium Commission to include other members of the radium family. It has been found that 1 curie of radon undergoes approximately $3.7 \times 10^{10}$ disintegration per second. Therefore, any member of the radium family in equilibrium with $1 \mathrm{~g}$ of radium undergoes the same number of disintegrations per second and may be called a curie of that isotope. The curie cannot be used correctly in any other sense, and attempts to extend it to other naturally or artificially active isotopes have been disapproved by the organization responsible for its definition.

The only other natural radioactive preparation that has been of much practical use is mesothorium. MsTh has a half-period of 6.7 years and therefore is about 250 times as active, weight for weight, as radium. Its disintegration products emit very energetic gamma rays. However, the half-period is too short for it to have permanent commercial value and its production is too small and subject to unforseen fluctuations. Its use in medical treatments and the production of self-luminous substances has never been very great.

The amounts of elements in the actinium family are too small and the half-periods are unsuitable for any commercial use. Natural uranium contains only about 0.7 percent of AcU.

\section{Artificial Radioactivity}

The production of radioactive isotopes from normally stable atoms was an outgrowth of experiments on transmutation of elements. Natural radioactivity had demonstrated that atoms in nature do undergo transmutation. As the nature of this process became better understood, it became evident that it might be possible to cause normally stable nuclei to undergo transmutations. Since the configuration of a stable nucleus represents a careful balance of powerful forces, the addition of another com- 
poinent with appropriate energy should upset this balance.

This view led to attempts to disrupt the nuclei of atoms by the use of alpha particles, the only particles available at the time with sufficient energy and in sufficient numbers to produce a detectable effect. Rutherford first succeeded in demonstrating the transmutation of a stable nucleus by bombarding nitrogen with these particles. Protons with energies much greater than those of the bombarding alpha particles were obtained by him, showing clearly that the bombarded nucleus had contributed some of its energy to these expelled protons.

The success of this experiment led many experimenters to investigate methods of producing bombarding particles in greater numbers than were available from naturally radioactive sources. Since it was presumed that any particle with sufficient energy to penetrate the nucleus might be able to cause disintegration, Cockcroft and Walton [16] undertook to accelerate protons, produced by ionizing hydrogen, through a potential difference of several hundred thousand volts. They found that when these protons impinged on a piece of lithium, alpha particles were released. By photographing the tracks of these particles in a Wilson chamber they demonstrated the nature of the particles and found that 2 alpha particles were ejected simultaneously in opposite directions in each disintegration.

Following the now common method of representation this reaction can be written

$$
{ }_{3} \mathrm{Li}^{7}+{ }_{1} \mathrm{H}^{1} \rightarrow{ }_{2} \mathrm{He}^{4}+{ }_{2} \mathrm{He}^{4}+Q,
$$

where the subscripts refer to the nuclear charge (=number of protons=atomic number of the nucleus). The superscripts refer to the mass number ( $=$ the sum of protons and neutrons in the nucleus). It is obvious from the results of this bombardment that the impinging proton was captured in the lithium nucleus and the resulting compound nucleus split into two alpha particles which, the experiment revealed, were ejected with considerable velocities, representing a correspondingly large amount of energy, $Q$, approximately $17 \mathrm{Mev}$.

It is of interest to inquire where this energy came from. Since it is much greater than that of the bombarding protons, it must have come from the nucleus. To understand this we must refer to the principle put forward by Einstein that energy and mass are equivalent, in accordance with the relation

$$
E=m c^{2},
$$

where $E$ is energy in ergs, $m$, the mass in grams, and $c$, the velocity of light. Therefore, to balance the masses in the equation representing this disintegration, the mass equivalent to the energy, $Q$, of the two alpha particles should be added. The masses of the nuclei entering into the reaction are known from the atomic weight and are given by $W\left(1.66 \times 10^{-2 t}\right)$ where $W=$ atomic weight, since the mass of an atomic weight of unity has been determined as $1.66 \times 10^{-2.4} \mathrm{~g}$. The atomic weight for lithium is 7.01784 , and for hydrogen is 1.00813. The sum gives a total nuclear mass of $(7.01818+$ $1.00813) \quad 1.66 \times 10^{-24}=1.33231 \times 10^{-23} \mathrm{~g}$. The atomic weight of helium is 4.00386 so that the nuclear mass of two alpha particles is: $2(4.00389) \times 1.66 \times 10^{-24}=1.32928 \times 10^{-23} \mathrm{~g}$. Therefore there are $(1.3323-1.32928) \times 10^{-23}=$ $0.00303 \times 10^{-23} \mathrm{~g}$ missing on the right-hand side of the equation. This mass represents $\left(0.00303 \times 10^{-23}\right) \quad c^{2} \mathrm{erg}=\left(0.00303 \times 10^{-23}\right)$ $8.9864 \times 10^{20}=2.75 \times 10^{-5}$ erg. Now, 1 erg $=$ $6.24 \times 10^{5}$ Mev. Therefore, $\left(2.75 \times 10^{-5}\right) 6.24$ $\times 10^{5}=17.2 \mathrm{Mev}$. Careful measurements of the total energies of the alpha particles emerging from this reaction gave $17.13 \pm 0.06 \mathrm{Mev}$ and $17.28 \pm 0.03 \mathrm{Mev}$. This experiment gives a complete verification of Einstein's postulate of the equivalence of mass and energy. This relation between mass and energy may be used to determine the change of mass involved in a disintegration from the measured release of energy or conversely to determine the release of energy to be expected when the masses are known. Whereas in most readily producible nuclear reactions energy is liberated, that is the particle or photon emitted has an energy greater than that of the incident particle, a few reactions have been observed in which energy is absorbed in the reaction. A typical example is

$$
{ }_{7} \mathrm{~N}^{14}+{ }_{2} \mathrm{He}^{4} \rightarrow{ }_{8} \mathrm{O}^{17}+{ }_{1} \mathrm{H}^{1},
$$

where the energy of the emergent proton is considerably less than that of the incident alpha particle.

\section{Disintegration by Protons}

The reaction of protons with lithium is one of a great number of disintegrations that have been observed when using protons as bombarding particles. Many of these produce a radioactive product such as

$$
{ }_{53} \mathrm{I}^{127}+{ }_{1} \mathrm{H}^{1} \rightarrow{ }_{54} \mathrm{Xe}^{127}+{ }_{0} n^{1},
$$

which yields a xenon isotope with a 34-day half-value period, with the liberation of a neutron. A type of proton reaction is represented by

$$
{ }_{26} \mathrm{Fe}^{57}+{ }_{1} \mathrm{H}^{1} \rightarrow{ }_{27} \mathrm{Co}^{58}+\gamma,
$$

resulting in a radioactive isotope of cobalt with half-period of 72 days and the liberation of a gamma ray. 


\section{Disintegration by Deuterons}

Bombardment of nuclei with deuterons also produces disintegrations. In many cases these reactions result in radioactive isotopes. The first result obtained with deuterons is represented by

$$
{ }_{3} \mathrm{Li}^{6}+{ }_{1} \mathrm{H}^{2} \rightarrow{ }_{2} \mathrm{He}^{4}+{ }_{2} \mathrm{He}^{4},
$$

which is similar to that obtained with ${ }_{3} \mathrm{Li}^{7}$ and protons.

When deuterons are used to bombard heavy hydrogen two different reactions are known:

$$
{ }_{1} \mathrm{H}^{2}+{ }_{1} \mathrm{H}^{2} \rightarrow{ }_{2} \mathrm{He}^{3}+{ }_{0} n^{1},
$$

and

$$
{ }_{1} \mathrm{H}^{2}+{ }_{1} \mathrm{H}^{2} \rightarrow{ }_{1} \mathrm{H}^{3 *}+{ }_{1} \mathrm{H}^{1} .
$$

The product of the first is a stable isotope of helium plus a neutron. The second gives a radioactive isotope of hydrogen, known as tritium, plus a proton.

Another type of reaction is represented by

$$
{ }_{21} \mathrm{Sc}^{45}+{ }_{1} \mathrm{H}^{2} \rightarrow{ }_{22} \mathrm{Ti}^{45}+2{ }_{0} n^{1},
$$

where two neutrons are released and a radioactive isotope of titanium of $3 \mathrm{hr}$ half-period is produced.

\section{Disintegration by Alpha Particles}

The development of the cyclotron in which very energetic alpha particles can be produced by accelerating helium nuclei to high velocities, has provided a much more abundant source of these particles than was available from naturally radioactive sources. Therefore a number of reactions that were uncertain or unknown before this method of acceleration was developed have been observed by the use of these particles.

A typical reaction is represented by

$$
{ }_{5} \mathrm{~B}^{10}+{ }_{2} \mathrm{He}^{4} \rightarrow{ }_{7} \mathrm{~N}^{13}+{ }_{0} n^{1},
$$

which produces radioactive nitrogen with the release of a neutron. One other type of reaction using alpha particles is represented by

$$
{ }_{46} \mathrm{Pd}^{103}+{ }_{2} \mathrm{He}^{4} \rightarrow{ }_{47} \mathrm{Ag}^{111}+{ }_{1} \mathrm{H}^{1},
$$

producing radioactive silver with the release of a proton.

\section{Disintegration by Neutrons}

Neutrons can be used to produce many more disintegrations than any other type of particle.
This can be ascribed to the absence of electrical charge, which makes it relatively easy for this particle to penetrate the strong electrical fields of the nucleus. In fact, neutrons with energies very near that of thermal agitation, $0.04 \mathrm{ev}$, are very effective in producing nuclear disintegration. Other reactions require neutrons with relatively high energies. One of the most common type of reactions with slow neutrons is represented by

$$
{ }_{79} \mathrm{Au}^{197}+{ }_{0} n^{1} \rightarrow{ }_{79} \mathrm{Au}^{198}+\gamma .
$$

The radioactive product of this reaction is an isotope of the stable gold nucleus with the mass number increased by unity. A gamma ray is released in the process. This type of reaction is very common and is the predominant type occurring in uranium piles, which are essentially sources of thermal neutrons.

Another interaction of neutrons with nuclei is represented by the typical reaction

$$
{ }_{28} \mathrm{Ni}^{58}+{ }_{0} n^{1} \rightarrow{ }_{28} \mathrm{Ni}^{57}+2{ }_{0} n^{1} .
$$

This reaction in general requires neutrons of fairly high energy. This is also true of reactions represented by

$$
{ }_{27} \mathrm{Co}^{59}+{ }_{0} n^{1} \rightarrow{ }_{26} \mathrm{Fe}^{59}+{ }_{1} \mathrm{H}^{1},
$$

and the somewhat rare reaction represented by

$$
{ }_{42} \mathrm{Mo}^{92}+{ }_{0} n^{1} \rightarrow{ }_{40} \mathrm{Zr}^{89}+{ }_{2} \mathrm{He}^{4} \text {. }
$$

\section{Disintegration by Photons}

Gamma rays of high energy are also capable of producing disintegration of the nucleus. When by absorption they give up energy to a nucleus the components become unstable, and disintegration results. This type of reaction was discovered by Chadwick and Goldhaber [17] in an experiment that can be represented by

$$
{ }_{1} \mathrm{H}^{2}+\gamma \rightarrow{ }_{1} \mathrm{H}^{1}+{ }_{0} n^{1} .
$$

Thus the incident gamma ray causes the deuteron to split into a proton and a neutron. Reactions of this kind have been found to be endothermic; that is, they proceed at the expense of the energy possessed by the incident gamma ray, and the mass of the end products is greater than that of the target nucleus. Consequently there is a threshold value for the energy of the photon below which the reaction cannot occur, which is set by the increase of mass. It is noteworthy that reactions of this type involve the transformation of the energy of electromagnetic radiation into matter, another example of the interchangeability of mass and energy. An ex- 
ample of the production of a radioisotope by this process is

$$
{ }_{47} \mathrm{Ag}^{107}+\gamma \rightarrow{ }_{47} \mathrm{Ag}^{106}+{ }_{0} n^{1} .
$$

The radioisotope ${ }_{47} \mathrm{Ag}^{106}$ emits a positron and decays to stable ${ }_{46}^{40} \mathrm{Pd}^{106}$ with a half-period of $24.5 \mathrm{~min}$.

\section{Alternate Methods for Producing a Given Radioisotope}

From the numerous ways in which radioisotopes can be produced, it is possible to select several nuclear reactions that give the same radioisotope. When the radioactive substance to be produced is required in a pure form, that is "carrier free," it is necessary to select a method of preparation that involves a transmutation; that is the atomic number of the target material differs from that of the radioisotope produced. The radioisotope then may be separated chemically from the target material in those cases where the yield is sufficient to permit such a procedure.

To illustrate the numerous ways for producing a single radioisotope, the known reactions for making ${ }_{9} \mathrm{~F}^{18}$, which has a half-period of $112 \min$ are

$$
\left.\begin{array}{l}
{ }_{9} \mathrm{~F}^{19}+\gamma \quad \rightarrow_{9} \mathrm{~F}^{18}+{ }_{0} n^{1}, \\
{ }_{9} \mathrm{~F}^{19}+{ }_{1} \mathrm{H}^{2} \rightarrow \rightarrow_{9} \mathrm{~F}^{18}+{ }_{1} \mathrm{H}^{3}, \\
{ }_{9} \mathrm{~F}^{19}+{ }_{0} n^{1} \rightarrow{ }_{9} \mathrm{~F}^{18}+2{ }_{0} n^{1}, \\
{ }_{8} \mathrm{O}^{18}+{ }_{1} \mathrm{H}^{1} \rightarrow \rightarrow_{9} \mathrm{~F}^{18}+{ }_{0} n^{1}, \\
{ }_{8} \mathrm{O}^{17}+{ }_{1} \mathrm{H}^{2} \rightarrow{ }_{9} \mathrm{~F}^{18}+{ }_{0} n^{1}, \\
{ }_{10} \mathrm{Ne}^{20}+{ }_{1} \mathrm{H}^{2} \rightarrow{ }_{9} \mathrm{~F}^{18}+2 \mathrm{He}^{4} .
\end{array}\right\}
$$

All six reactions yield the same radioisotope of fluorine with the same period, decaying by positron emission to ${ }_{8} \mathrm{O}^{18}$, one of the stable isotopes of oxygen.

\section{Decay of Artificially Radioactive Substances}

Whereas the radioactive decay of artificially active isotopes obeys the same exponential laws discovered in connection with natural radioactivity the predominant modes of decay show some noticeable differences; very few of the artificial isotopes decay by alpha-ray emission, and the majority undergo a beta-ray type of decay, characterized by the emission of a continuous spectrum of beta-ray energies with a definite upper limit. Long chains of radioactive elements are absent among these isotopes, except in the case of a number of fission products.

\section{Positron Emission}

The radioactive decay of the silver isotope, ${ }_{47} \mathrm{Ag}^{106}$ illustrates a type of activity not known among natural radioactive substances that is fairly common among the artificially induced activities. This nucleus decays by emitting positrons in a continuous spectrum similar to that of beta-ray emitters. Therefore it goes over to ${ }_{46} \mathrm{Pd}^{106}$. These positrons are assumed to be created in the nucleus at the time of emission much in the same way that beta particles are produced. Neither are now regarded as constituents of the nucleus. Wherever positrons are present, annihilation radiation is produced as these particles combine with electrons to produce photons. This gamma radiation has an energy corresponding to the amount of mass that has disappeared, twice the rest mass of the electron. Since two photons of equal energy are produced for each electron and positron annihilation, the energy of each photon is $0.51 \mathrm{Mev}$.

\section{Electron Capture}

Artificial isotopes also decay by capturing an orbital electron, usually from the $K$-level, and so this process is commonly called $K$-capture. The net result is similar to that produced by positron emission, the product nucleus having one less unit of nuclear charge. However, no annihilation radiation is present. Instead, X-radiation, corresponding to the level from which the electron was captured, appears. This radiation is characteristic of the product atom. It frequently happens that a radioisotope decays partly by electron capture and partly by positron emission, both processes yielding the same product.

\section{Isomeric Transitions}

Among artificial radioisotopes, frequent examples occur in which an isotope, consisting of nuclei of the same mass and the same charge, decays with two different periods. This situation is prevalent among isotopes that emit positrons. Many decay partly by positron emission and partly by electron capture. It may also occur in a different way, represented by the only example among the natural radioelements in the case of $\mathrm{UX}_{2}$ and $\mathrm{UZ}$. This anomaly is now explained by assuming that $\mathrm{UX}_{2}$ is an excited metastable state and UZ the ground state of the ${ }_{91} \mathrm{~Pa}^{234}$ nucleus. The greater number $(99.85 \%)$ of these nuclei assumed to have been left in the excited state $\left(\mathrm{UX}_{2}\right)$ decay directly to the ground state of ${ }_{92} \mathrm{U}^{234}$ (UII) emitting a beta ray of approximately $2.3 \mathrm{Mev}$. The remaining fraction emit a gamma ray, brings them to the ground state of ${ }_{91} \mathrm{~Pa}^{234}$ (UZ), 
which then decays by emitting a beta ray and one or more gamma rays to the ground state of ${ }_{92} \mathrm{U}^{234}$. The period for the first mode is $1.14 \mathrm{~min}$

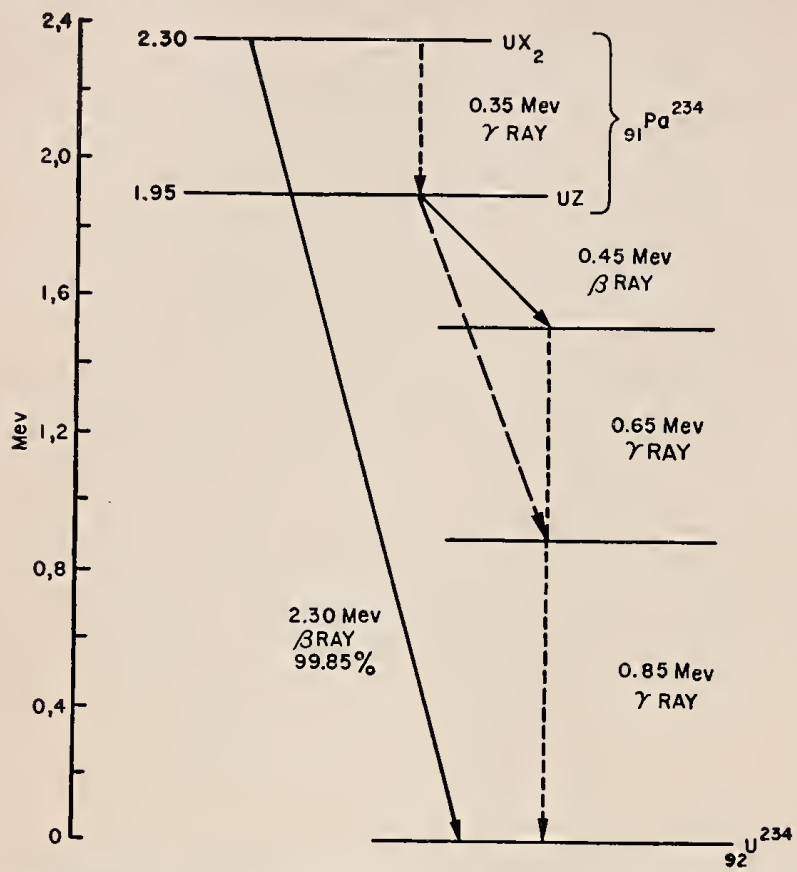

FIgURE 32. Disintegration scheme showing isomeric transition in disintegration of ${ }_{91} \mathrm{~Pa}^{234}$.

(Bradt, et al. Helv. Phys. Acta, 18, 259 (1945)

and for the second $6.7 \mathrm{hrs}$. Considerable experimental evidence is available to support this point of view, which represents an entirely logical explanation. An approximate representation of this disintegration is shown in figure 32.

A summary of some of the more common nuclear reactions is given in the chart in figure 33 , where the nuclear charge is represented by $Z$ and the mass number by $A$. Thus ${ }_{Z} X^{A}$ represents any isotope. In addition to those reactions listed in figure 33, there are a few less common ones. For example, for some of the lighter atoms, deuteron bombardment results under favorable conditions in the capture of the deuteron and the ejection of a tritium nucleus, $t$. Alpha particle bombardment in rare instances results in the capture of the alpha particle and the simultaneous release of a proton and a neutron.

\begin{tabular}{|c|c|c|c|c|c|c|c|}
\hline \multirow{2}{*}{$\begin{array}{l}\text { INITIAL } \\
\text { NUCLEUS }\end{array}$} & \multicolumn{2}{|c|}{$\begin{array}{l}\text { PARTICLE } \\
\text { OR } \\
\text { RADIATION }\end{array}$} & \multirow{2}{*}{$\begin{array}{l}\text { RESULTANT } \\
\text { NUCLEUS }\end{array}$} & \multirow{2}{*}{\begin{tabular}{|l} 
INITIAL \\
NUCLEUS
\end{tabular}} & \multicolumn{2}{|c|}{$\begin{array}{l}\text { PARTICLE } \\
\text { OR } \\
\text { RADIATION } \\
\end{array}$} & \multirow{2}{*}{$\begin{array}{c}\text { RESULTANT } \\
\text { NUCLEUS }\end{array}$} \\
\hline & IN & OUT & & & IN & OUT & \\
\hline${ }_{2} X^{A}$ & $\gamma$ & $n$ & & ${ }_{z} X^{A}$ & $d$ & $2 n$ & \\
\hline${ }_{z} X^{A}$ & $n$ & $\gamma$ & & ${ }_{z} X^{A}$ & d & $\alpha$ & \\
\hline${ }_{z} X^{A}$ & $n$ & $2 n$ & & ${ }_{z} X^{A}$ & $p$ & $\gamma$ & $z+1 X^{A+1}$ \\
\hline${ }_{2} X^{A}$ & $n$ & p & $z-1^{\prime}$ & ${ }_{z} X^{A}$ & P & $n$ & ${ }_{2+1} X^{A}$ \\
\hline${ }_{z} X^{A}$ & $n$ & $\alpha$ & & ${ }_{2} X^{A}$ & $p$ & $\alpha$ & $z_{z-1} X^{A-3}$ \\
\hline$z X^{A}$ & $d$ & $n$ & $z_{z+1} X^{A+1}$ & ${ }_{z} X^{A}$ & $\alpha$ & $n$ & $z+2 X^{A+3}$ \\
\hline$z X^{A}$ & $d$ & $P$ & & ${ }_{z} X^{A}$ & $\alpha$ & P & $z+1 X^{A+3}$ \\
\hline
\end{tabular}

FIGURE 33. Chart showing some typical nuclear reactions for producing artificial radioisotopes.

These reactions are represented by the symbols $(d, t)$ and $(a, n p)$, respectively. Evidence also exists of $(a, a),(n, n)$ and $\left(e^{-}, n\right)$ reactions, the latter representing the release of a nuclear neutron by electron bombardment.

\section{Equipment for Producing Artificially Radioactive Isotopes}

The rapid progress in extending the number and increasing the quantity of artificial radioactive isotopes has been the result of the development of means of accelerating particles. Most nuclear reactions produce greater yields, and some reactions only occur, when the bombarding particles have high energies. Whereas Joliot and Curie discovered artificial radioactivity with relatively simple equipment in which neutrons were produced by bombarding beryllium with alpha particles, the amounts of radioactive substances produced were very small and just barely detectable by the radiation that tr:ey emitted. For production on a larger scale, several types of very elaborate equipment have been devised.

\section{Voltage Multiplier}

On the first successful equipment for accelerating particles and producing nuclear transmutation was developed by Cockcroft and Walton [16]. They used an electron voltage multiplier for this work. The operation of such a circuit can be explained by reference to figure 34. Condenser $C_{1}$ is charged to the potential, $V$, of the battery permanently connected across it. When the switch is in the position shown by solid lines, $C_{2}$ is also charged to this potential. When the switch is thrown to the dotted position, $C_{3}$ is in parallel with $C_{2}$, and they come to the common potential of $V / 2$, assuming they have equal capacitance. Repeating the switching 


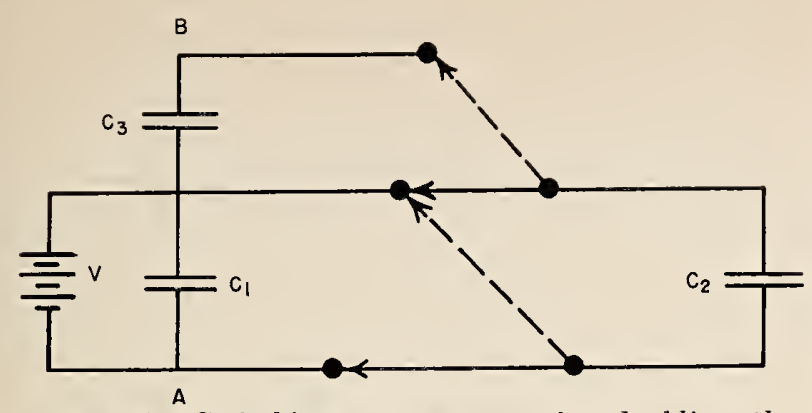

Figure 34. Switching arrangement for doubling the voltage from a source.

$V=$ battery, the source of voltage, $C_{1}, C_{2}, C_{3}=$ condensers.

operation brings $C_{3}$ to a potential of $3 \mathrm{~V} / 4$. After several successive repetitions, the potential across $C_{3}$ is practically equal to $V$, and the potential from $A$ to $B$ is $2 V$. This is the principle of the voltage doubler that can be extended to quadrupling the voltage. In actual practice the switching process is accomplished by thermionic rectifiers that provide for unidirectional current when an alternating potential is applied to the condensers. A diagram of the voltage quadrupler used by Cockcroft and Walton is shown in figure 35 , where $a, b, c$, and $d$ are the rectifier tubes. In the loop of the circuit, including the secondary of the transformer, the condenser, $C$, and the rectifier $A$, the potential of the point $B$ varies periodically with respect to point $A$ from zero to $2 \mathrm{~V}$, where

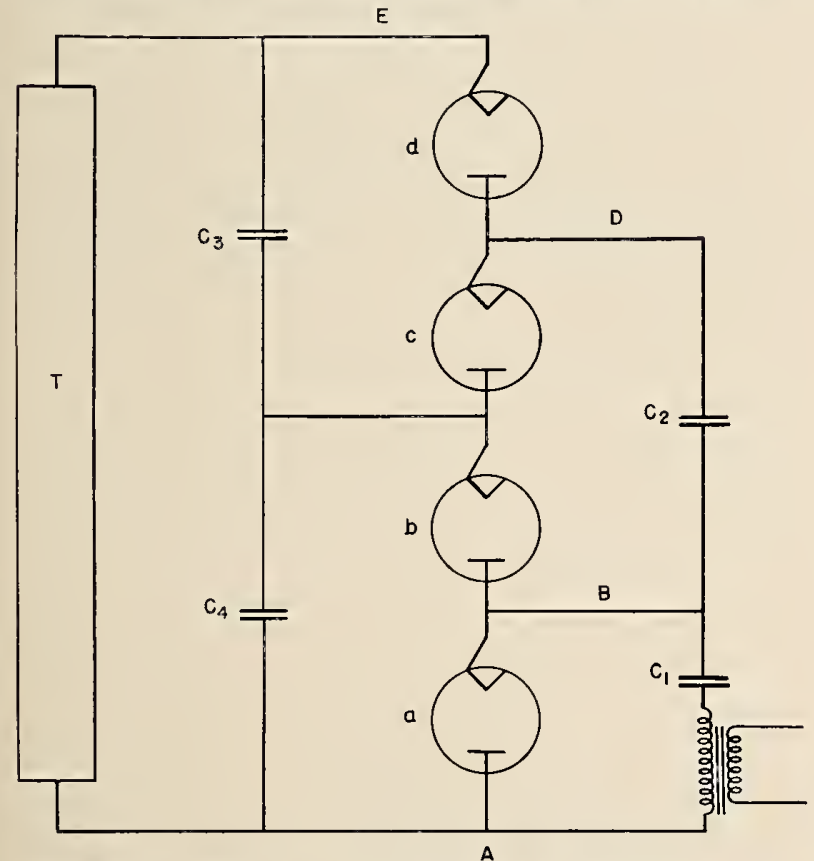

FiguRe 35. Diagram of the voltage quadrupling circuit of Cockroft and Walton. $T=$ Discharge tube.

$V$ is the peak voltage of the secondary. As the voltage of $B$ rises to $2 \mathrm{~V}$, the plate of rectifier $b$ becomes positive with respect to its filament, and $b$ conducts so that condenser $c$ shares its charge with $C_{4}$. Thus points $B$ and $C$ are each at potential $V$ above $A$. On the reverse half of the cycle, the potential of point $B$ falls below that of $A$, resulting in the lowering of potential of point $D$. Thus $C$ is at a higher potential than $D$, so that rectifier $C$ becomes conducting, and $C_{4}$ shares its charge with $C_{2}$, and $C$ and $D$ come to the same potential. On the next rise of the potential wave across $A B$, the potential of $D$ again rises. Since the potential of $C$ with respect to $A$ has not changed and is at the same potential as $E$, the rise in the potential of $D$ raises it above that of $G$, whereupon rectifier $d$ conducts, causing $C_{2}$ to share its charge with $C_{3}$. The final result is that condenser $C_{3}$ and $C_{4}$ are each charged to a potential of $2 \mathrm{~V}$. Thus the voltage across $T$ is $4 V$, neglecting leakage losses and current drain in $T$.

\section{Cascade Transformers}

High alternating voltages can be produced by transformers connected in a cascade arrangement such that the insulation of each transformer must withstand only the voltage developed in its secondary. Figure 36 is a diagram of this method of connecting transformers.

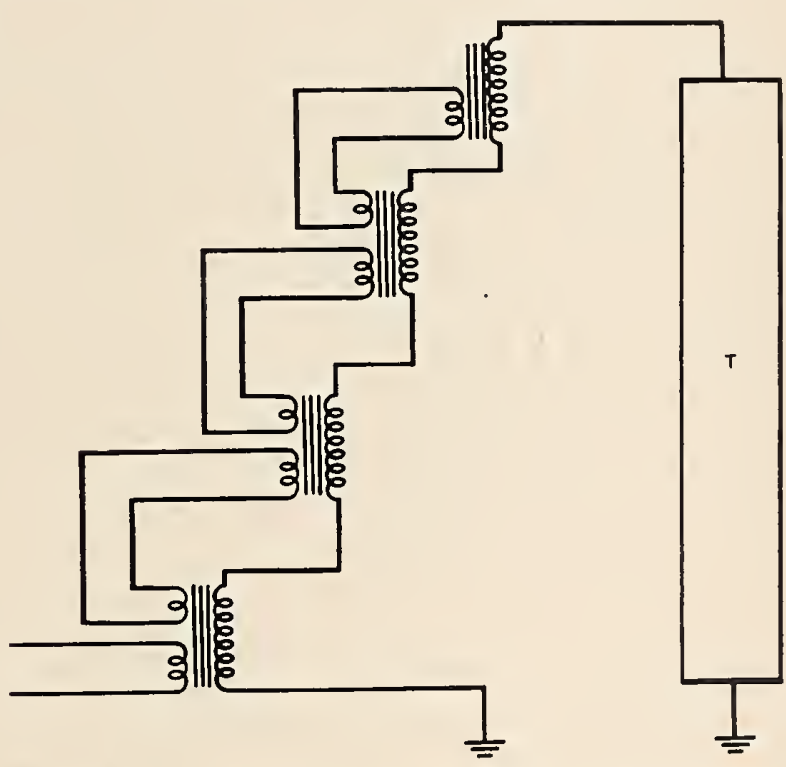

Figure 36. Diagram of connection of transformer's in cascade for producing high alternating voltages.

Each transformer has an additional primary winding that energizes a primary winding on the succeeding transformer. Since this arrangement applies an alternating potential to the accelerator tube, ions are accelerated only on the positive half of the potential wave. This cascade arrangement of transformers was developed by Sorensen and has been used by Lauritsen and Crane in disintegration experiments. 


\section{Van de Graaf Generator}

This generator is based on the behavior of a static charge of electricity applied to a hollow metallic sphere. The natural repulsion of charges of like sign forces the charge to the outer surface. Therefore charge can be introduced into the interior of such a sphere until the potential of the sphere reaches the point at which the electric field in the gas surrounding the sphere causes the gas to become con-

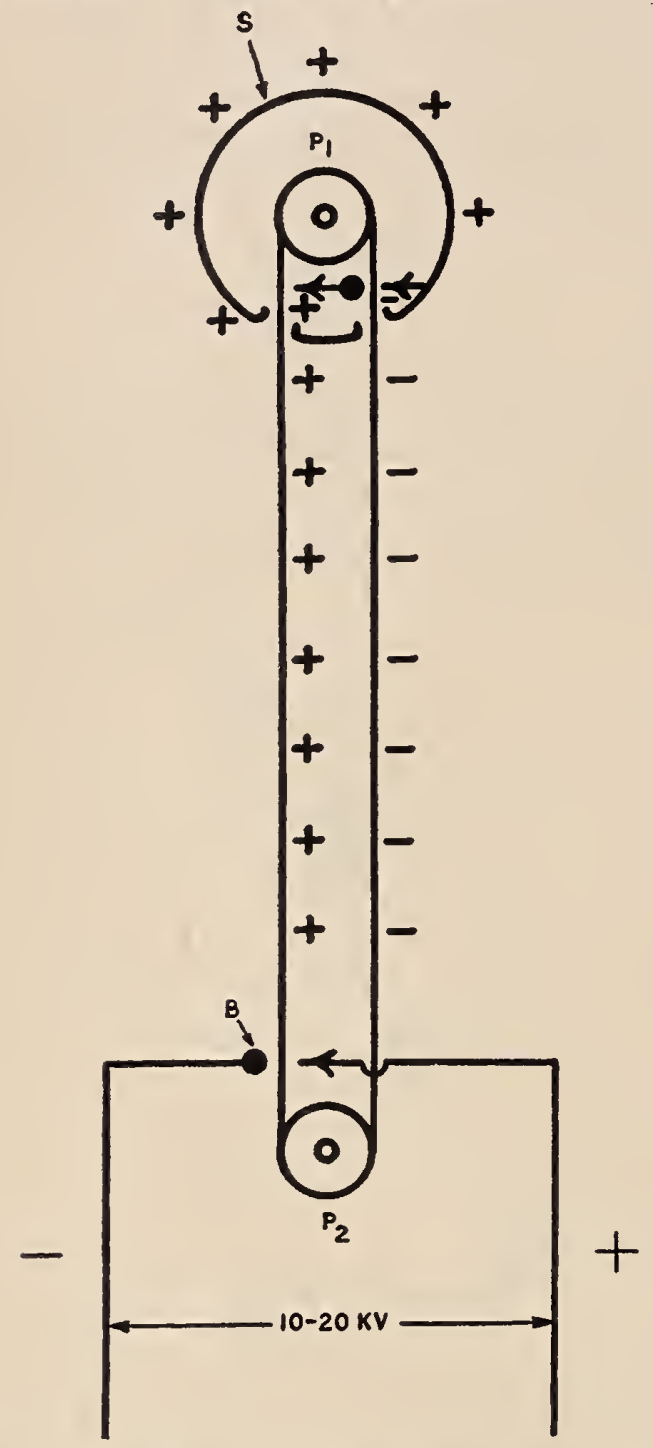

FIGURE 37. Diagram of Van de Graaf direct-current generator.

$P_{1}, P_{2}=$ pulleys; $S=$ spbere; $B=$ inductor plate and comb.

ducting and the insulating property of the gas is destroyed.

An arrangement for charging a large metallic sphere to a potential of several million volts is shown diagrammatically in figure 37.

The sphere, $S$, has a pulley, $P_{1}$, mounted at the center, which carries a long belt of some in- sulating substance, such as rubber. The lower loop of the belt passes around the pulley of $P_{2}$, of a motor. At $B$ an inductor plate and comb are arranged to spray a positive charge of electricity on the rapidly moving belt. Inside the sphere a comb electrode removes the positive charge from the belt. The efficiency of this machine can be increased by arranging an inductor plate on the inner side of the descending belt in the sphere with a comb electrode attached to the sphere. Then negative charge sprays onto the descending belt.

\section{Linear Accelerator}

This name is applied to an arrangement of tubular electrodes mounted in an evacuated tube on a common axis with an alternating potential of high frequency and opposite sign applied to successive electrodes. The lengths of the electrodes are selected for the frequency used, so that a particular group of ions will move from the center of one electrode to that of the next in a half-period of the oscillating voltage. At each transit from one electrode to the next, the ions acquire energy proportional to the peak voltage of the oscillator and thus increase their velocity. Therefore, each successive electrode must be longer than the preceding one, until relativistic velocities are reached. In this region the increase in mass due to increase in velocity is appreciable, and the velocity changes slowly.

Recent developments in the utilization of oscillators of very high frequency have extended the usefulness of the linear accelerator. It may be possible to accelerate ions by this principle to energies of the order of a billion electron volts. Figure 38 is a diagram of this type of accelerator.

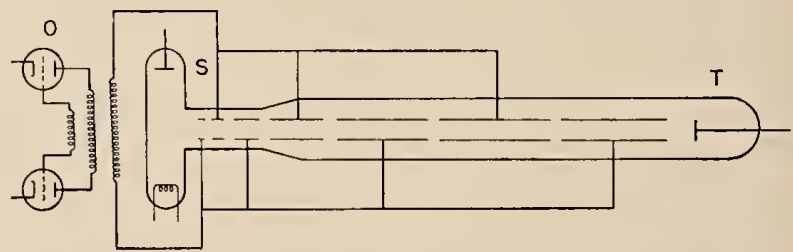

FIGURE 38. Diagram of a linear accelerator. $O=$ high-frequency oscillator; $S=$ source of ions, $T=$ receiving electrode.

Since increasing the dielectric strength of the gas surrounding the sphere raises the breakdown potential, models have been constructed in which the sphere is inside a tank that can be filled with special gases at several atmospheres pressure. Conversely, the size of the equipment for a given potential can be reduced by this arrangement. Herb and coworkers [18] have constructed a $2.4 \mathrm{Mev}$ generator in a tank containing a mixture of air and freon $\left(\mathrm{CCl}_{2} \mathrm{~F}_{2}\right)$, which reduces the number of free electrons in 
the gas. By using three concentric electrodes and reducing the separation, he was able to increase the voltage to $4.5 \mathrm{Mev}$ in the same size of equipment. This improvement is based on the fact that at high pressure the dielectric strength of air is greater in a short path than over a long one.

\section{Cyclotron}

The cyclotron was developed by Lawrence [19] and consists of a vertical magnetic field to cause ions to travel in horizontal circular paths inside hollow circular electrodes that alternate in potential, thus acquiring additional velocity in each half-circle. As the velocity of the ions increases, the diameter of the circle in which they travel increases so that the time of transit remains constant. The ultimate energy acquired depends on the strength of the magnetic field and the diameter of the chamber in which the ions circulate, as well as on the magnitude of the oscillating potential. Figure 39 is

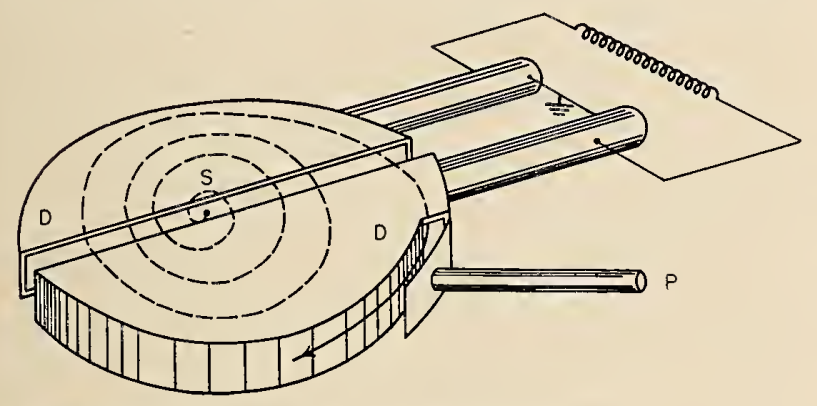

Figure 39. Diagram of the cyclotron dees. $D, D=$ dees; $S=$ ion source; $P=$ deflecting plate.

a diagram of the cyclotron showing the two hollow electrodes, or "dees", and the general method for applying the oscillating potential. This device is particularly suited to the acceleration of deuterons. Heavy hydrogen is admitted to the evacuated chamber containing the dees and ionized at the center. The beam of deuterons emerges tangentially at the periphery. Although targets may be bombarded to produce transmutations inside the low-pressure chamber, for many purposes it is desirable that the target be outside the chamber to reduce loss of products through evaporation.

\section{Betatron}

Electrons may be accelerated to energies col'responding to $100 \mathrm{Mev}$ by using the principle on which the induction of currents by an alternating magnetic field is based. A device operating on this principle was first described by Kerst [20]. If we consider an annular volume that is evacuated and contains electrons and apply a rapidly increasing magnetic field perpendicular to the plane of the annulus over an area entirely within the annulus, the electrons tend to rotate about the annulus on a rapidly expanding circle as they increased in velocity and escape tangentially. However, if the electrons are subjected to a magnetic field in the same direction over the entire area of the annulus, they will be deflected toward the center. If this field is of exactly the right magnitude the electrons will then travel in a circular orbit within the annulus. This requires that the magnetic induction, $B$, over the annulus increase at the same rate as the change of momentum of the electron due to the change of flux within the annulus. This will give an orbit of constant radius $r$. The average electromagnetic force on the electrons, $E$, is equal to $\left(\phi_{1}-\phi_{2}\right) / t$ where $\phi_{1}-\phi_{2}$ represents the change of flux in time $t$. The tangential electrical field on the electron in orbit of radius $r$ is $\epsilon E / 2 \pi r$ and the force on the electron is $\epsilon e$. Therefore, the acceleration $a$ is given by

$$
\mathrm{a}=\frac{\epsilon e}{m}=\frac{e E}{m_{2} \pi r}=\frac{e}{m} \quad \frac{\phi_{2}-\phi_{1}}{2 \pi r t} .
$$

If $\left(\phi_{2}-\phi_{1}\right) / t=10^{9} / \mathrm{sec}$ for a radius of $10 \mathrm{~cm}, a$ has a value of $2.8 \times 10^{13} \mathrm{~cm} / \mathrm{sec} / \mathrm{sec}$. This corresponds to $\phi_{2}-\phi_{1}=1,000,000$ maxwells in $1 / 1,000$ sec. The distance traveled by the electron in $1,000 \mathrm{sec}$ is then $1.4 \times 10^{7} \mathrm{~cm}$, or $2.24 \times 10^{5}$ revolutions. Since $E$ is $10 \mathrm{v}$ in this case, the energy gained would be $2.24 \mathrm{Mev}$ neglecting the relativistic increase in mass of the electron.

The condition for an orbit of constant radius is $B_{2}-B_{1}=\left(\phi_{2}-\phi_{1}\right) / 2 \pi r^{2}$ where $B_{2}-B_{1}$ is the change of magnitude of induction. In practice the saturation of the iron used in producing the magnetic induction produces a condition within the quarter cycle of the oscillating magnetic field, during which electrons are accelerated, where the change in $B$ does not satisfy the condition for a constant radius of orbit. The orbit begins to decrease in size. A target is mounted just inside the constant orbit, so that

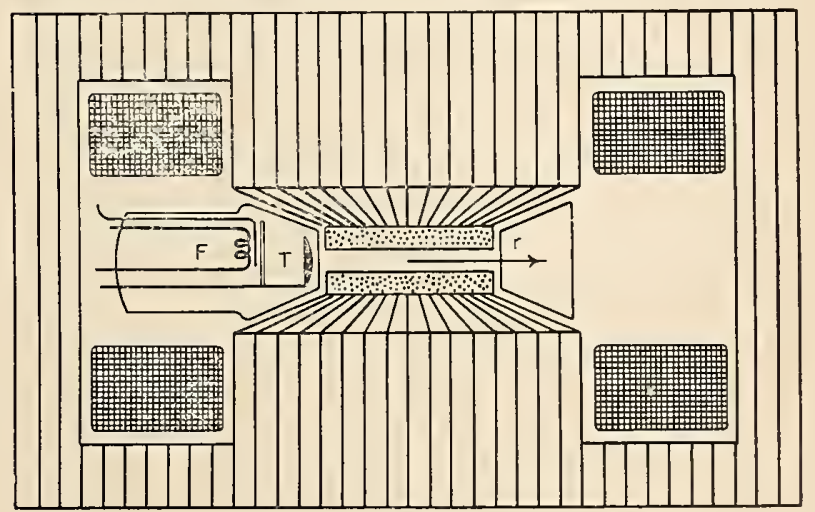

Figure 40. Diagram of the betatron. $F=$ filament serving as source of electrons; $T=$ target; $r=$ radius of electron orbit. 
the electrons strike it as the size of the orbit shrinks, thus producing electromagnetic radiation of very high energy. A diagram of the betatron is shown in figure 40 .

\section{Synchrotron}

To increase the energy acquired by ions in making one turn in the betatron, and also to permit the use of positive ions, the principles of the betatron combined with those of the cyclotron have been applied to a device called the synchrotron, suggested by MacMillan [21]. As proposed, the machine would have dees operated at a potential of high frequency with the perpendicular magnetic field also alternating. Thus ions undergo a dual acceleration, and energies as high as $10^{3} \mathrm{ev}$ have been predicted from a device of this type.

With the exception of the betatron, all the devices mentioned may be used in one of two ways to produce artificially radioactive substances. They may be used to accelerate particles that impinge on a target of the element to be disintegrated, or a target may be selected that emits neutrons when struck by the bombarding particles and the neutrons used to produce radioactivity in elements exposed to them.

\section{Uranium Reactors}

The uranium piles developed during the war provide intensities of slow neutrons much greater than previously available. A pile of moderate power using ordinary uranium may have a flux, $n v$, of the order of $10^{12} / \mathrm{sec} \mathrm{cm}^{2}$ over a considerable area. Therefore, these piles are the most prolific sources of artificially radioactive isotopes produced by the $(n, \gamma)$ reaction that have been devised.

These piles consist of uranium distributed through a moderator with some means of carrying away the heat generated by the absorption of the radiations in the pile structure. The operation depends basically on the fission of uranium atoms that capture neutrons and the fact that a chain reaction can be produced, utilizing the neutrons that are released in the fission process. $U^{235}$ is the only natural isotope of uranium that undergoes fission for a large proportion of the neutrons captured. At the same time more than two neutrons are released for each fission and are available to sustain the chain reaction.

To maintain the chain reaction, the loss of neutrons that do not produce fission of $U^{235}$ must be reduced to a small proportion of the total number. This means that impurities that have high capture cross sections must be removed from the moderator and the uranium. Pure graphite or heavy water will serve as moderating material. However, in the case of graphite, impurities must be reduced well below concentrations usually found in socalled "pure" graph- ite. Also, losses occur by neutrons diffusing outside the pile. The proportion lost can be reduced by increasing the size of the pile; since the number of neutrons produced goes up as the volume, and those lost by diffusion are proportional to the surface area. Neutrons are also lost from the chain reaction by capture in $U^{238}$ when ordinary uranium is used. The percentage lost in this way remains constant for a definite ratio of $U^{235}, U^{238}$ as exists in natural uranium. Therefore as the size of a chain reactor is increased, a size will be found where the excess neutrons released in the fission process just equals the number of neutrons lost by diffusion and capture. This is the critical size, and the reaction is selfsustaining at this stage.

In practice, piles are constructed that are definitely larger than the critical size so that in addition to producing fission products from the uranium, other material may be placed in the pile and made radioactive by neutron capture. Since piles above critical size would increase rapidly in activity, all working piles must be controlled. This is done by introducing control rods containing material such as boron or cadmium, which have high-capture cross sections for slow neutrons. These rods are used to keep the pile just at the operating level. As substances are introduced for activation the control rods are removed by an amount to restore equilibrium.

The reactivity of a pile is measured in terms of the period. If the pile is not in equilibrium, the neutron density will rise or fall exponentially with a definite period $T$, defined by relation $I \propto e^{ \pm 1 / T}$, where $I$ is the intensity or neutron density. This leads to the measurement of the reactivity in terms of reciprocal hours so that a change in $1 / T$ of one per hour corresponds to one unit of reactivity defined as 1 in hour. Therefore the control rods of a given pile can be calibrated in in hours and the available reactivity determined from their position.

Although most piles so far constructed are slow neutron piles and therefore can produce radioactive isotopes predominantly by $n, \gamma$ reactions, consideration has been given to the development of piles operating with fast neutrons that should extend the types of activations. Enriched piles using $U^{235}$ or $P u^{239}$ have been designed, which provide much higher densities of neutrons in smaller volumes than can be produced with natural uranium. Higher specific activities by the $n, \gamma$ process can therefore be produced in them, or a given specific activity produced in shorter time.

\section{Fission Products}

In addition to isotopes that may be made radioactive by slow neutron bombardment, uranium piles are prolific sources of fission 
products, which frequently can be separated with a high specific activity, that is, without the addition of a carrier. These isotopes are located near the middle of the periodic table and range from germanium to europium. The halfperiods are, in general, shorter than a few days. Numerous decay chains, or families, have also been discovered among fission products.

\section{RADIOACTIVE RADIATIONS}

\section{Neutrons}

Although the neutron is one of the more recently discovered particles, it is one of the most fundamental in current view of radioactivity. The conversion of a neutron into a proton is associated with the emission of the beta ray in those radioactive disintegrations that proceed by this method. Since the neutron has no electric charge and therefore is incapable of ionizing atoms directly, its identity was difficult to establish even when experiments had been performed that clearly indicated a type of radiation previously unknown. Bothe and Becker [22] observed a very penetrating radiation when beryllium was bombarded by alpha rays from polonium. This was interpreted as gamma radiation of very high energy, since the new radiation was very penetrating and produced very little ionization. Subsequently Chadwick [23] was able to show by recoil experiments that the new radiation consisted of a neutral particle with a mass approximately equal to that of the hydrogen nucleus.

\section{Production of Neutrons}

Since the discovery of the neutron, this particle has been recognized as a unit in the structure of atomic nucleus, the number in each nucleus being of the same order as the number of protons. Therefore neutrons are produced by the disintegration of nuclei by artificial means. The nuclear reaction by which neutrons were discovered is represented by

$$
{ }_{4} \mathrm{Be}^{9}+{ }_{2} \mathrm{He}^{4} \rightarrow{ }_{6} \mathrm{C}^{12}+{ }_{0} n^{1},
$$

where one neutron is released for each atom undergoing disintegration. The neutron thus released will have a kinetic energy determined by the known energies and masses involved in the reaction. Protons, deuterons, and photons may all produce nuclear disintegrations that release neutrons.

\section{Mass of the Neutron}

Eiarliest estimates of the mass of the neutron were made by measuring the energies of hydrogen and nitrogen nuclei when neutrons collided with them. Although sufficient for identifying the mass of the neutron, the method is not capable of an accuracy better than a few percent. However, nuclear reactions in which nevtrons are produced offer a possibility of a much higher accuracy. For example, Chadwick and Goldhaber [24] found that neutrons are produced by irradiating heavy hydrogen by $2.62-\mathrm{Mev}$ gamma rays from $\mathrm{ThC}^{\prime \prime}$ according to the reaction

$$
{ }_{1} \mathrm{H}^{2}+\gamma \rightarrow{ }_{1} \mathrm{H}^{1}+{ }_{0} n^{1} .
$$

The energy of the proton produced by this reaction can be measured, and the neutron should have approximately the same kinetic energy since it has nearly the same mass. Therefore, the equivalent masses in the above reaction can be written in for all terms except the mass of the neutron. The kinetic energy of the neutron and proton has been determined as $0.45 \mathrm{Mev}$. The above reaction in mass units, using the equality $1 \mathrm{Mev}=1.07 \times 10^{-3}$ atomic mass units,

deuteron + gamma $r a y=$ proton $+\mathrm{KE}+$ neutron,

$$
2.01473+0.00279=1.00757+0.00096+M_{n},
$$

where $M_{n}$ is the mass of the neutron and is therefore equal to 1.00899 , which is probably accurate to within 0.005 percent. Since the mass of the neutron is larger that that of the proton by an amount corresponding to about $1.3 \mathrm{Mev}$, the transition from a neutron to a proton releases energy. Therefore the neutron is unstable with respect to the proton and should emit a beta ray in the transition. Numerous attempts have been made to detect this radioactivity of the neutron without definite success to date, largely because of the very short half-period for this transition.

It is of interest to note that the neutron also has a magnetic moment in spite of the difficulties of ascribing such a property to a neutral particle. This moment can be calculated by assuming a deuteron to be a combination of a proton and a neutron. The deuteron has a moment of 0.8565 and the proton 2.7896 nuclear magnetons. Therefore, the neutron should have -1.9331 nuclear magnetons, indicating that the relative directions of the magnetic moment and the angular momentum are opposite to those for the proton and deuteron. Measurement has given -1.9103 for the magnetic rnoment of the 
neutron in fairly close agreement with the simple representation outlined.

\section{Measurement of Neutrons}

Neutrons can be detected only by indirect processes in which they produce secondary radiations, which can produce ionization. Furthermore, neutrons of high energies react quite differently from those of low energy. Therefore in measuring neutrons, it is desirable to know the number and the velocity of each. In practice it is customary to select various ranges of velocity and determine the number in each group. This is accomplished in some cases by filtration, which removes all neutrons below a certain velocity by use of a filter of a substance such as cadmium, which has a very high cross section for thermal neutrons.

\section{Fast Neutrons}

Swift neutrons are most readily measured by permitting them to strike hydrogen atoms and convert them into protons in the recoil reaction. The ionization produced by these protons is readily measured and is proportional to the number of swift neutrons producing them. The usual arrangement is to use a hydrocarbon, such as paraffin, as a target for the neutrons. The protons ejected from the paraffin are measured in an ionization chamber, Wilson chamber, or other device sensitive to ionizing particles. It is frequently desirable to know the distribution of the energies of swift neutrons from a particular source. One method of obtaining the relative numbers of various energies is to measure the energies of individual recoil protons in a parallel plate ionization chamber with a linear pulse amplifier. Such a device is insensitive to slow neutrons. A typical arrangement is shown in figure 41.

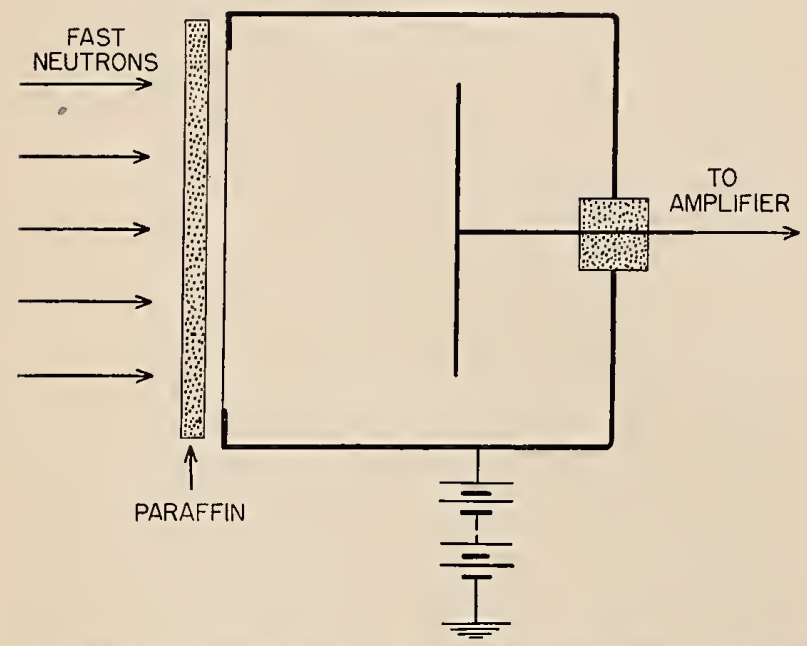

FIgURE 41. Chamber for counting fast neutrons by means of recoil protons from a layer of paraffin.
It should be noted that any device operating on the principle of detecting recoil protons detects only those fast neutrons moving in a direction to impel the protons into the sensitive region of the detector.

\section{Slow Neutrons}

Slow neutrons are those with energies below about $100 \mathrm{ev}$, hence this is a general term for a considerable group. Thermal neutrons, those having the velocities of thermal agitation, have velocities determined by the temperature in accordance with the relation $V^{2}=(2 k T) / m$, and the standard temperature is $20^{\circ} \mathrm{C}$ or $293^{\circ} \mathrm{K}$. Since $k=1.3708 \times 10^{-16}$ erg per degree absolute per particle and $m$, the mass of the neutron, is $1.67 \times 10^{-24} \mathrm{~g}$, the standard velocity of 2,200 meters/sec corresponds to an energy of $0.025 \mathrm{ev}$.

Slow neutrons may be measured by a variety of methods, all based on their property of producing disintegration of nuclei to which they readily penetrate.

\section{Counting Alpha Particles Produced. by Neutron-Induced Disintegrations}

A number of the lighter elements, of which boron, and particularly the natural isotope ${ }_{5} \mathrm{~B}^{10}$, is a good example, emit an alpha particle when a slow neutron is captured by the nucleus. These alpha particles have a range of a few millimeters in air and therefore can be detected and counted by proportional counters or shallow ionization chambers. One method is to fill a conventional tubular proportional counter with $\mathrm{BF}_{3}$ gas. Since boron has a very large capture cross section for slow neutrons, this makes a very sensitive device. Another method is to coat one plate of a parallel plate ionization chamber with a thin coating of metallic boron or the oxide and to amplify the alpha particle pulses by means of a linear amplifier. Methane or purified nitrogen in the chamber improves the sharpness of the ionization pulses, making them more suitable for amplification.

\section{Counting Gamma Rays Resulting from Neutron Capture}

When slow neutrons are captured to produce nuclear disintegration, gamma rays are emitted in the process. Therefore, if a Geiger-Müller gamma-ray counter is covered with a layer of metallic cadmium, for example, which also has a large cross section for slow neutron capture, the counters will respond to the gamma rays produced by the slow neutrons. The cadmium can be relatively thin, since a sheet $0.5 \mathrm{~mm}$ thick is virtually opaque to thermal neutrons. The activity induced in the cadmium by the disintegration increases the background of the counter so that this arrangement cannot be used for accurate measurements. 


\section{Induced Activities}

The amount of the radioactive isotope produced by bombardment with slow neutrons is proportional to the number of neutrons that strike the sample. Therefore, by exposing a foil to the neutrons to be measured for a definite time and measuring the activity-counting the rate of emission of beta rays, for example-at a definite time subsequent to the exposure, the number of neutrons that were captured in the exposure interval can be determined. The halfperiod of the induced activity must be known as well as the capture cross section of the isotope from which the foil is made. The relation for this computation is given by eq 11 , where $n v N_{\sigma}$ is sustituted for $n_{0}, n=$ number of neutrons per cubic centimeter, $v=$ their velocity, $N=$ number of atoms in foil, and $\sigma=$ their cross section. $P$, the number of radioactive atoms present in the foil at time, $t$, subsequent to exposure to a neutron flux of $n v$ for a time $T$ is given by

$$
P=\frac{n v N \sigma}{\lambda}\left(1-e^{-\lambda T}\right) e^{-\lambda t},
$$

where $\lambda$ is the decay constant of the radioactive isotope formed by neutron capture. In practice two activities may be induced in the same foil, as, for example, when the element from which the foil is made consists of two isotopes in nature. If one of the activities has a much shorter period than the other, its effect can be eliminated experimentally by waiting a short time for the short activity to decay before measusing the radioactivity of the foil. In attempting absolute measurements of $n v$ by this method, it must be noted that the cross section used must correspond to the energies of the neutrons that are being measured.

Frequently it may be desirable to use a number of different foils with resonances for neutrons of different energies to determine the relative number of neutrons at corresponding energies.

\section{Cross Sections for Reactions with Neutrons}

When neutrons impinge on a group of atomic nuclei, the number of those that react is expressed in terms of the cross section of the particular nuclei for the neutrons in question. The cross section of a nucleus is expressed in square centimeters, representing the effective target area of the nucleus for the neutron. The symbol $\sigma$ is used to denote this quantity. When there are $n$ neutrons per cubic centimeter with velocity $v$, the number striking a square centimeter per second is $n v$, the neutron flux. If there are $N$ atoms per square centimeter of the target element, then the number of reactions per second is given by $n v N_{\sigma}$. A convenient unit for the cross section is $10^{-24} \mathrm{~cm}^{2}$. A specific cross section may be assigned to each type of interaction for a given nucleus. For example, a neutron may react with a nucleus by undergoing (1) scattering, either elastic or inelastic, or by (2) being captured or absorbed. Further distinction may be made by specifying the angle of scattering or the specific results of the capture that may result in emission of radiation of particles from the bombarded nucleus. Therefore, a number of subscripts may be applied to distinguish the process to which it applies. For example $\sigma_{a}$ is usually used to designate the total cross section of a nucleus for neutron capture and $\sigma_{e}$ for the cross section that leads to a radioactive product. Similarly, $\sigma_{s}$ is used to denote the total scattering cross section. Fundamentally $\sigma$ refers to a single nucleus so that a distinction must also be made between isotopic cross section and the atomic cross section per atom of the natural element for those elements that are composed of more than one isotope in nature.

The cross section not only depends on the nucleus but also on the energy of the neutron. In the region of slow neutrons the cross section may vary very markedly with the change in energy of the neutrons.

The measurement of cross sections under various conditions requires the selection of neutrons with energies distributed over a narrow region, since no sources of monoenergetic neutrons are available. Various methods have been used to obtain such segregation of neutrons with respect to their energies. The velocity selector in which the detector is placed at a definite distance from the source is one example. The detector and source are modulated at a known frequency so that the detector is sensitive for a definite interval after the release of neutrons from the source. The neutrons detected are then those that have velocities equal to $d / t$, where $d$ is the separation between source and detector, and $t$ is the time between release and detection of the neutrons. This method is limited to relatively slow neutrons. $\left(E<10^{2} \mathrm{ev}\right)$. A more common method for separating neutrons according to velocity, is by filtration. By proper selection of filters, various velocities of neutrons that correspond to resonance.levels in the filters may be removed. When only one resonance level is present, the cross section $\sigma$ $(E)$ as a function of the energy of the neutron, $E$ is given by the Breit-Wigner formula [25]

$$
\sigma(E)=\frac{E_{r}}{E^{1 / 2}} \frac{\sigma_{0}}{1+\left(\frac{E-E_{r}}{\Gamma / 2}\right)^{2}}
$$


where $\sigma_{0}$ is the cross section at $E_{r}$, the energy of resonance level, and $\Gamma$ is the total width of the resonance level. When the width of the level is large compared with the energy of the neutrons, the Breit-Wigner formula takes the form $\sigma(E)=\mathrm{K} 1 / v$. An absorber for which these conditions held will absorb slow neutrons much more strongly than those of higher energy and therefore will increase the average energy of the neutron beam. When the resonance level of the filter is near thermal energies the filter will have a very high cross section in the thermal region, and drop quite sharply above the resonance energy. Cadmium, which is an example of this behavior, has a cross section greater than $10^{-20} \mathrm{~cm}^{2}$ for all energies below $0.3 \mathrm{ev}$ and practically zero for energies above $0.5 \mathrm{ev}$. Therefore, less than 1 percent of neutrons of energy below $0.1 \mathrm{ev}$ will be transmitted by a sheet of cadmium $0.5 \mathrm{~mm}$ thick. Neutrons that are thus absorbed in cadmium have been called " $C$ " neutrons. In determining cross sections of elements for $C$ neutrons, the method of taking the "cadmium difference" is used. If $N_{0}$ is the number of incident neutrons and $N_{x}$ is the number transmitted by a thickness, $x$, of the element under investigation, $N_{c}$ the number transmitted by the cadmium layer alone, and $N_{c+x}$ the number transmitted by the sample plus the layer of cadmium, the cross section for $C$ neutrons is calculated from the relation for $I$, the number transmitted

$$
I=e^{-N} \sigma_{c} x=\frac{N_{x}+N_{c+x}}{N_{0}-N_{c}},
$$

where $N$ is the number of atoms per square centimeter in the layer of the element under investigation, and $\sigma_{c}$ is the cross section for $C$ neutrons.

Crystal diffraction offers the possibility of obtaining beams of neutrons that are quite monoenergetic. The deBroglie wavelength $\lambda=$ $h / m v$, for slow neutrons is comparable with the atomic spacing in crystals, and they will be scattered in accordance with the Bragg relation, $\sin \theta=n \lambda / 2 d$. This method can be used for neutrons with energies between 0.02 and $0.30 \mathrm{ev}$ by using crystals available at present and also offers a means of measuring cross sections at various small increments of energies of the neutrons over this region.

Cross sections for neutron capture may be obtained by measuring the radioactivity produced in those cases where a radioactive isotope results from the capture. This cross section is frequently referred to as the excitation cross section $\sigma_{e}$. It is most conveniently determined when the element under investigation can be irradiated for a long period compared with the half-period of the radioisotope produced. Equilibrium then exists between the rate of capture and the rate at which radioactive nuclei of the radioisotope are decaying. This saturation activity gives directly the rate of capture, and $\sigma$ may be computed from the relation

$$
\sigma_{e}=\frac{n_{c}}{(n v) N}
$$

where $n_{c}$ is the number of captures per second, $n v$ is the neutron flux, and $N$ is the number of nuclei per square centimeter exposed to the beam of neutrons.

It should be noted that measurement of neutrons transmitted through an element gives data from which the total cross section may be computed. To determine the absorption cross section, additional measurements must be made to determine the scattering cross section so that it may be subtracted if only the absorption cross section is desired. The scattering cross section can be measured by using a well-collimated beam and measuring the neutrons scattered at a given angle. Absolute values may be determined by comparison with the scattering from some substance such as graphite, for which the scattering cross section is accurately known.

In the use of neutrons for the production of artificially active isotopes, the excitation cross section is of greatest interest. For practical purposes the value of this cross section for the neutrons from a particular source is required. The knowledge of this factor is required to compute yields of radioactive elements to be anticipated.

\section{Radioactivity Produced by Neutrons}

The simplest reaction of a neutron with a nucleus is its capture by the nucleus with the emission of gamma radiation. The absence of electrical charge accounts in part for the relative ease with which slow neutrons are captured by nuclei, since potential barriers of the nucleus do not tend to exclude them. Another factor is that at velocities near that of thermal agitation the neutrons undergo numerous collisions with nuclei. Resonance phenomena also play an important role, since the actual velocities of so-called slow neutrons extend over a considerable range, and therefore the chance of a neutron having an energy at some particular time corresponding to a nuclear level is enhanced. Slow neutron reactions result in no change in the charge of the nucleus and the resulting radiative nucleus is an isotope of the target nucleus. This, of course, limits the specific activity that can be produced to a definite fraction of the total number of atoms exposed. This fraction depends on the cross section, the intensity of the neutrons, the time of irradia- 
tion, and the half-value period of the radioactive product.

Neutrons can also produce reactions in which the composite nucleus formed by the capture of a neutron ejects a particle, such as a proton, alpha particle, or a pair of neutrons. These reactions, with rare exception, require neutrons of relatively high energies for much the same reasons given in the similar case for protons. When charged particles are emitted, the effect of neutron bombardment resonance appears to be less important than the delivery of sufficient energy by the incident particle to permit the ejected particle to escape through the nuclear potential barrier.

\section{Protons}

The proton is the nucleus of the hydrogen atom and therefore has one elementary unit of positive charge. The mass number of the proton (oxygen $=16.000$ ) is 1.0076 , corresponding to $1.6725 \times 10^{-24} \mathrm{~g}$, and it has a magnetic moment of 2.7896 nuclear magnetrons. It is regarded at present as one of the fundamental units of nuclear structure, with all nuclei made up of one or more protons corresponding to the nuclear charge and sufficient neutrons to account for the mass. Protons have never been observed as a product of radioactive disintegration except as constituents of alpha particles. On the other hand, they frequently are expelled from the compound nucleus formed by bombardment of stable nuclei resulting in nuclear disintegration and the production of artificially radioactive isotopes.

\section{Production of Protons}

Protons are produced as the result of nuclear integrations. A typical reaction is represented by

$$
{ }_{5} \mathrm{~B}^{11}+{ }_{2} \mathrm{H}^{4} \rightarrow{ }_{6} \mathrm{C}^{14}+{ }_{1} \mathrm{H}^{1} .
$$

By varying the number and energy of the incident alpha particles, protons of different energies can be produced in varying numbers. However, the intensities of proton beams available by this means are very weak, and measurements can be made only under favorable experimental conditions with sensitive detectors. For investigations of protons where larger intensities are required and for their use in nuclear disintegrations, these particles are produced by ionizing hydrogen and accelerating the hydrogen nuclei in one of the several devices for accelerating positive ions, for example, a highvoltage accelerator.

Figure 42 is a diagram of a typical lowvoltage arc arranged to produce protons from hydrogen. This gas is introduced into the bulb surrounding the cylinder at a pressure of 0.1 to $0.5 \mathrm{~mm} \mathrm{Hg}$ pressure. The filament serves as a source of electrons that are accelerated by a potential difference of 30 to $40 \mathrm{v}$ between the filament and the anode. The ion gun is several hundred volts negative with respect to the anode. Protons are admitted to the accelerating tube through a capillary that permits the arc to operate at a considerably higher pressure than that of the accelerating tube. Proton currents of the order of milliamperes can be obtained by such methods.

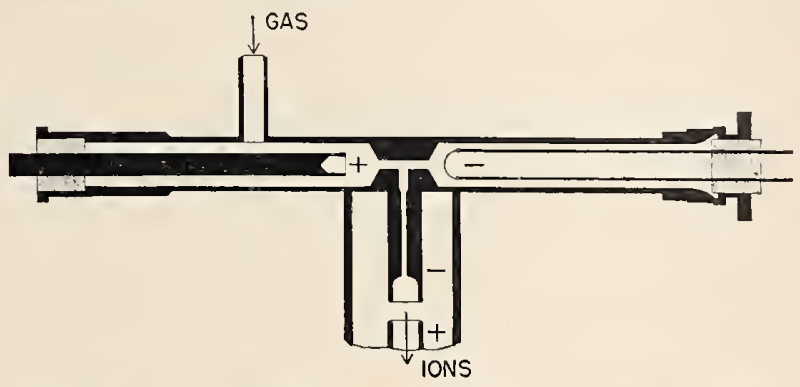

FIGURE 42. Positive ion source for producing protons.

\section{Defection and Measurement of Protons}

Relatively intense beams of protons may be measured in terms of ionization produced in any of the conventional types of ionization chambers. The windows of such chambers must be thin enough to admit a relatively large fraction of the protons. Protons of $0.5 \mathrm{Mev}$ are completely absorbed in aluminum $0.006 \mathrm{~mm}$ thick, and 1.0-Mev protons are absorbed in $0.015 \mathrm{~mm}$. Individual protons of moderate energy may be detected in any of the devices that will respond to ionizing particles. As their energy increases, ionization per centimeter of path decreases to the extent that single protons having energies greater than $2 \mathrm{Mev}$ are difficult to detect. The average number of ions produced per centimeter in air at atmospheric pressure at $2 \mathrm{Mev}$ is about 8,000 , whereas at $7 \mathrm{Mev}$ this number has decreased to about 3,000 . This may be compared with the ionization produced by an alpha particle that is of the order of 50,000 ion pairs per centimeter under similar conditions. The relatively low penetrating power of these particles does not permit the use of Geiger-Müller counters, since windows thin enough to permit them to enter will not withstand the required difference in pressure. The device most successful in detecting these particles is some form of proportional counter, in which the ionization produced by each is multiplied by impact ionization in the gas of the counter. The decrease of ion pairs per centimeter with increasing energy complicates the problem of estimating the energy from the size of pulse produced in a proportional counter. This estimate can only be made when the full 
range of each proton is completely absorbed in the counter. Since the range of $2 \mathrm{Mev}$ protons is about $6.5 \mathrm{~cm}$, it is difficult for average counters to absorb the total range of protons of higher energy. Figure 43 shows the variation of the range of protons with energy for air and aluminum.

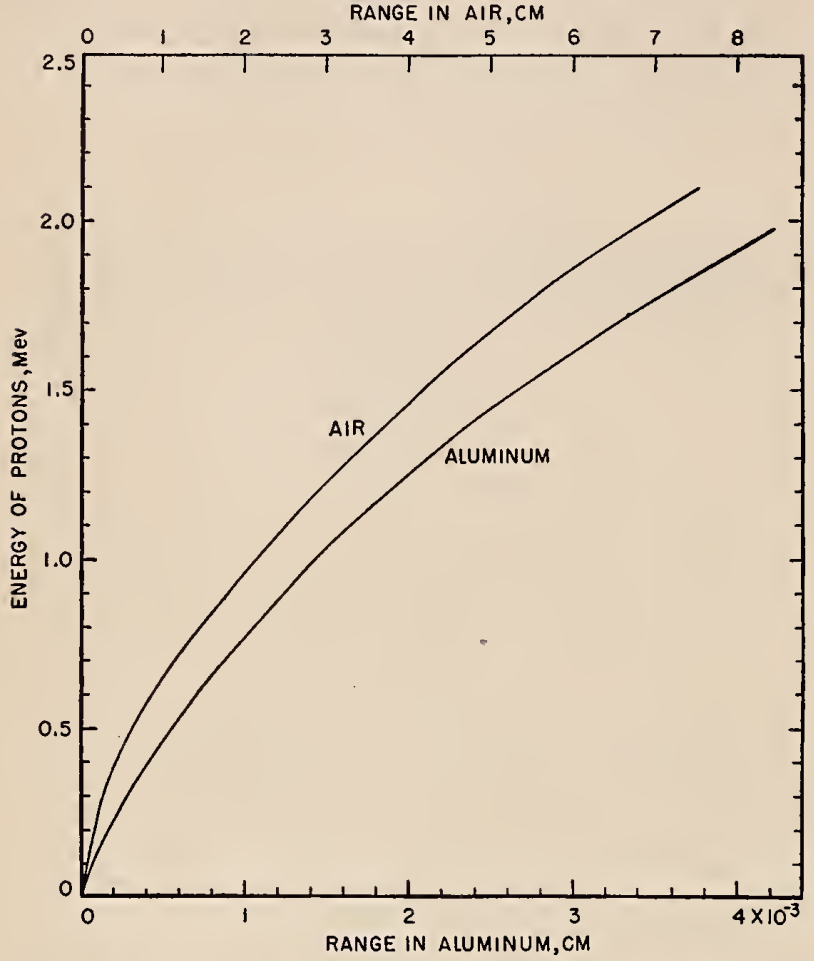

FigURE 43. Graph showing ranges in air and aluminum of protons of various energies up to $2 \mathrm{Mev}$.

\section{Radioactivity Produced from Proton Bombardment}

It has been observed that protons with energies as low as $0.02 \mathrm{Mev}$ can produce disintegration. However, such disintegrations do not result in all cases in the formation of a radioactive nucleus. When the energy of the bombarding proton is gradually increased at some particular energy, depending on the isotope under bombardment, radioactive nuclei are produced. This means that the energy of the incident proton corresponds to a resonance level of the nucleus of the bombarded isotope. The protons of this energy are captured, forming a compound nucleus that in the simplest case radiates energy in the form of gamma rays leaving a new nucleus, that is radioactive. Many such resonance levels have been observed for a single nucleus.

When the energy of the bombarding protons is increased, a different type of reaction may occur in which the proton is captured but a neutron is emitted from the composite nucleus. This reaction usually requires energies is excess of $2 \mathrm{Mev}$. The high threshold for this process is required because the neutron has more mass than the proton, and the radioactive nucleus also is heavier than the stable nucleus from which it is formed. This additional mass must be supplied in the form of kinetic energy of the incident proton. It is interesting to note also that this type of reaction results in a radioactive nucleus that decays by the emission of a positron. In general, no two stable isotopes of the same nuclear mass have been observed with a difference of nuclear charge of one unit. The capture of a proton and emission of a neutron leaves the nucleus with the same mass number but one additional unit of charge. This unstable nucleus emits a positron and thus returns to the original stable nucleus. An example is

$$
\left.\begin{array}{c}
{ }_{13} \mathrm{Al}^{27}+{ }_{14} \mathrm{H}^{1} \rightarrow \mathrm{Si}^{27} \rightarrow \mathrm{Si}_{13}^{27}+{ }_{0} n^{1}, \\
\mathrm{Al}^{27}+\beta^{+} .
\end{array}\right\}
$$

As the energy of the bombarding proton is increased above the excitation threshold for the emission of a neutron, the yield of radioactive atoms increases rapidly until a saturation yield is obtained that has been observed to occur at about $10 \mathrm{Mev}$. The curve shown in figure 44 illustrates the variation of yield with the energy of the incident proton.

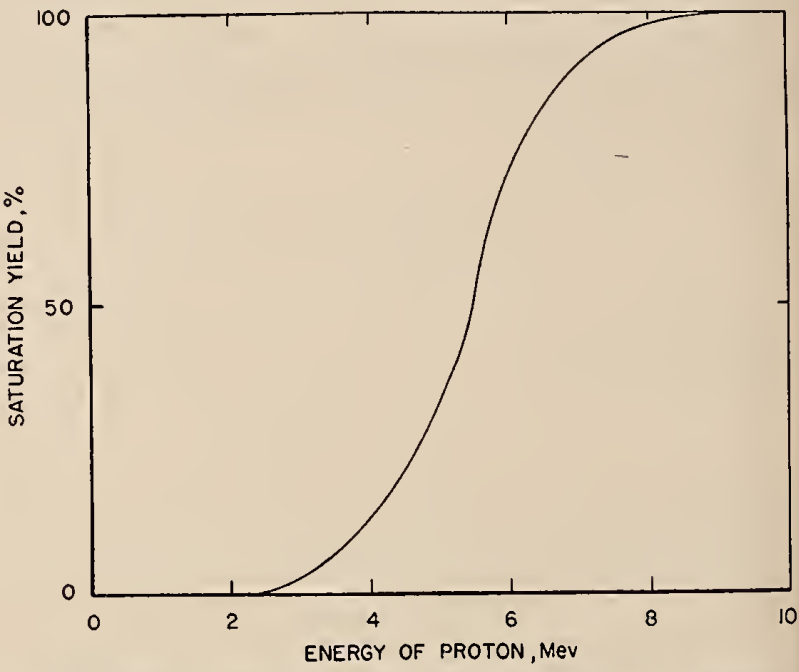

FiguRe 44. Graph showing the percentage of saturation yield of neutrons for protons of various energies impinging on the target.

A third typical reaction observed when protons of high energies are used in bombardment is represented by the capture of the proton and the emission of an alpha particle. Since the alpha particle must escape through the potential barrier of the compound nucleus, this explains qualitatively why the process requires the incident particle to have a relatively high energy. 


\section{Deuterons}

A discrepancy between the atomic weight of hydrogen as measured by the mass spectrograph and the chemical combining weight of the element led to the suggestions that a small amount of an isotope with a nuclear mass double that of the common variety is present in nature. Spectroscopic examination of samples of fractionally distilled liquid hydrogen made by Urey and collaborators [26] revealed lines in the spectrum assignable to a hydrogen atom of mass 2 . This observation was confirmed by the measurement of the mass of hydrogen atoms in such fractions by Bleakney [27] by using the mass spectrograph. The relative difference in mass between these two isotopes is so large that fairly simple methods of evaporation, diffusion, or electrolysis suffice to separate the 0.018 percent of this heavy isotope present in natural hydrogen.

The deuteron is the nucleus of the heavy hydrogen atom and may be prepared from these atoms by ionization. The deuteron has a mass of 2.0147 and a magnetic moment of $0.75 \mathrm{nu}-$ clear magnetrons. The combination of proton and neutron forming the deuteron has a binding energy of $2.2 \mathrm{Mev}$. Therefore, in energetic nuclear reactions there is always the possibility that the deuteron will be disassociated, setting a neutron free in the reaction. Recalling that the neutron can readily penetrate the nuclear potential barrier of the target atom, this may explain the relatively high efficiency of deuterons with energies greater than its binding energy in producing nuclear disintegrations.

\section{Deuteron Reactions}

One of the simplest nuclear reactions involving deuterons occurs when deuterons are bombarded by deuterons. The target consists of frozen heavy water in this experiment, and neutrons are produced in great numbers, even when the energy of the bombarding deuteron is as low as $0.1 \mathrm{Mev}$. The reaction is represented by

$$
{ }_{1} \mathrm{H}^{2}+{ }_{1} \mathrm{H}^{2} \rightarrow{ }_{2} \mathrm{He}^{3}+{ }_{0} n^{1},
$$

in which 0.0034 mass units disappear corresponding to $3.16 \mathrm{Mev}$, which appears as kinetic energy of the particles that are produced by the reaction. Similarly, Be and Li release neutrons when bombarded by deuterons. The reaction with lithium provides neutrons with energies as high as $25 \mathrm{Mev}$ for incident deuterons of $10 \mathrm{Mev}$.

When deuterons penetrate a nucleus with sufficient energy, a proton may be emitted from the compound nucleus instead of a neutron, as occurs at lower energies. The probability for the ejection of the charged particle increases with the energy. However, the probability for the ejection of a neutron under these circumstances also increases. In a few cases where these relative probabilities can be measured for the same nucleus, it has been found that the probability for ejecting a neutron increases proportionately more rapidly than for the ejection of a proton. This is regarded as substantiating the Oppenheimer-Phillips [28] hypothesis that the deuteron disintegrates in the reaction, so that only the neutron penetrates the nucleus, and the proton is not ejected from the nucleus but is repelled before it actually enters.

A third reaction that has been observed (when a stable nucleus is bombarded by deuterons) is the emission of an alpha particle. This may also be regarded as a competing reaction with the $(d, n)$ process. Cork and Halpern [29] have investigated the relative

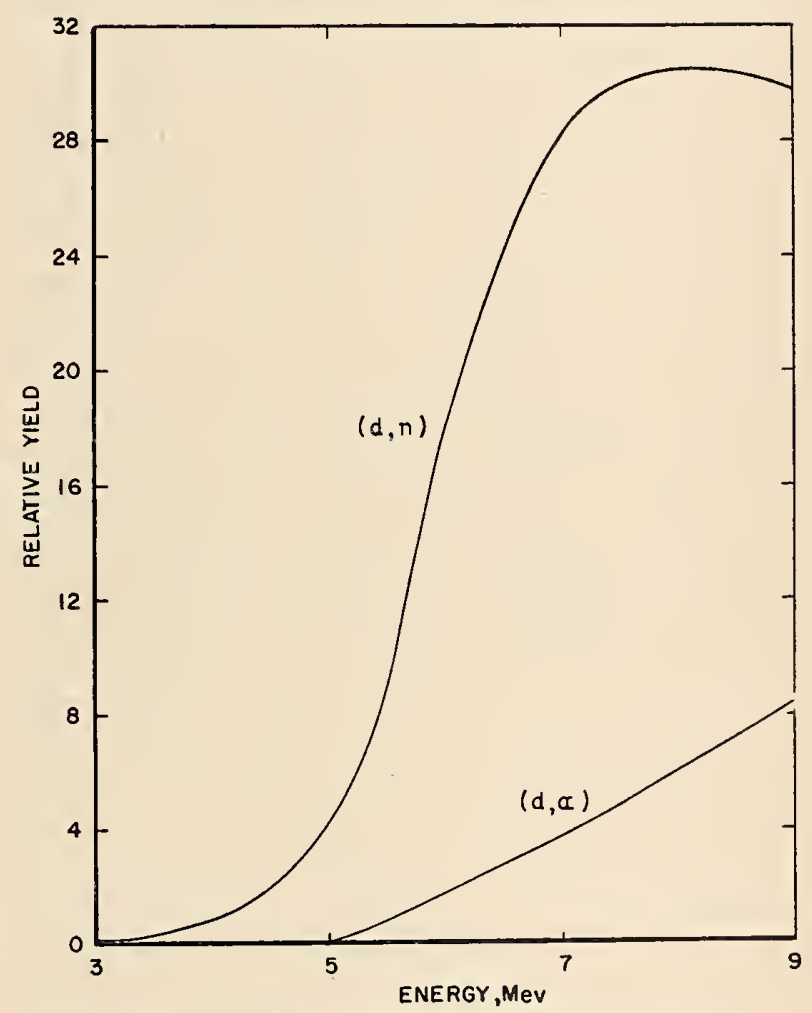

FIGURE 45. Graph showing the relative yields of neutrons and alpha particles for various energies of incident deuterons on an iron target.

probabilities of these two reactions for the ${ }_{26} \mathrm{Fe}^{54}$ nucleus. The $(d, a)$ process results in a radioactive manganese isotope ${ }_{25} \mathrm{Mn}^{52}$, and the $(d, n)$ reaction produces a radioactive cobalt isotope ${ }_{27} \mathrm{Co}^{55}$. By varying the energy of the incident deuterons and determining the relative amounts of the two products, the relative cross sections for the two reactions in a single nucleus were determined. The excitation curves are shown in figure 45 where the $(d, n)$ reaction 
begins at about $3 \mathrm{Mev}$ and the $(d, a)$ at slightly less than $5 \mathrm{Mev}$, with the $(d, n)$ reaching a saturation value at about $7.5 \mathrm{Mev}$, whereas the yield from the $(d, a)$ reaction continues to rise steadily up to the maximum energy of deuterons available, $9 \mathrm{Mev}$.

A fourth process observed in deuteron bombardment is the emission of two neutrons. This reaction, $(d, 2 n)$ may be regarded as comparable with the emission of two neutrons under neutron bombardment $(n, 2 n)$. Both constituents of the deuteron penetrate the nucleus in this reaction however, since the proton of the combination is captured yielding a product with one unit greater atomic number than that of the target nucleus. The reaction has been observed only in connection with energetic deuterons.

\section{Alpha Porticles}

Rutherford and Royds [30] proved that alpha particles are the nuclei of atoms of helium by examining the optical spectrum of the gas formed when alpha particles are trapped in an evacuated tube. The doubly ionized helium atoms recapture their orbital electrons when their velocities have been reduced to values near those of thermal agitation, resulting in atoms of helium. These particles, therefore, have the mass of the helium nucleus and carry two elementary positive charges. The value of $e / m$ for alpha particles was determined by application of electric and magnetic fields simultaneously to produce deflections in opposite directiuns, adjusting these fields to produce a net deflection of zero. Under these conditions $\mathrm{Hev}=E e$, where $H$ is the magnetic field and $E$ the electric field, $\epsilon$ the charge and $v$ the velocity of the particles. This gives the velocity as $v=E / H$, which can be used in the relation for the magnetic deflection alone, $\mathrm{Hev}=m v^{2} / r$, to determine $e / m=$ $E / H^{2} r$. The early measurements of this quantity were difficult to interpret since velocities, charge, and mass were initially unknown. When particles of single velocity were used, a value of 4,300 emu per gram was obtained, which is quite close to the most accurate value to date of 4,813 [31]. The value of $\mathrm{e} / \mathrm{m}$ for helium determined by the mass spectrograph is within a fraction of a percent of the same value, given by Birge [32] as $4822.3 \pm .05$.

\section{Range of Alpha Porticles}

Since alpha particles are emitted in groups with all particles in the group having the same initial energy, the distance that the particles in a particular group travel before losing sufficient kinetic energy that they no longer produce ionization is approximately the same. This distance measured in centimeters is the range and is usually specified for air at normal pressure and temperature. The ranges of alpha particles are most readily determined by ionization methods. The procedures and precautions required in such measurements are illustrated by the measurements of Holloway and Livingston [33] by using a shallow ionization chamber. A differential chamber was used by Lewis

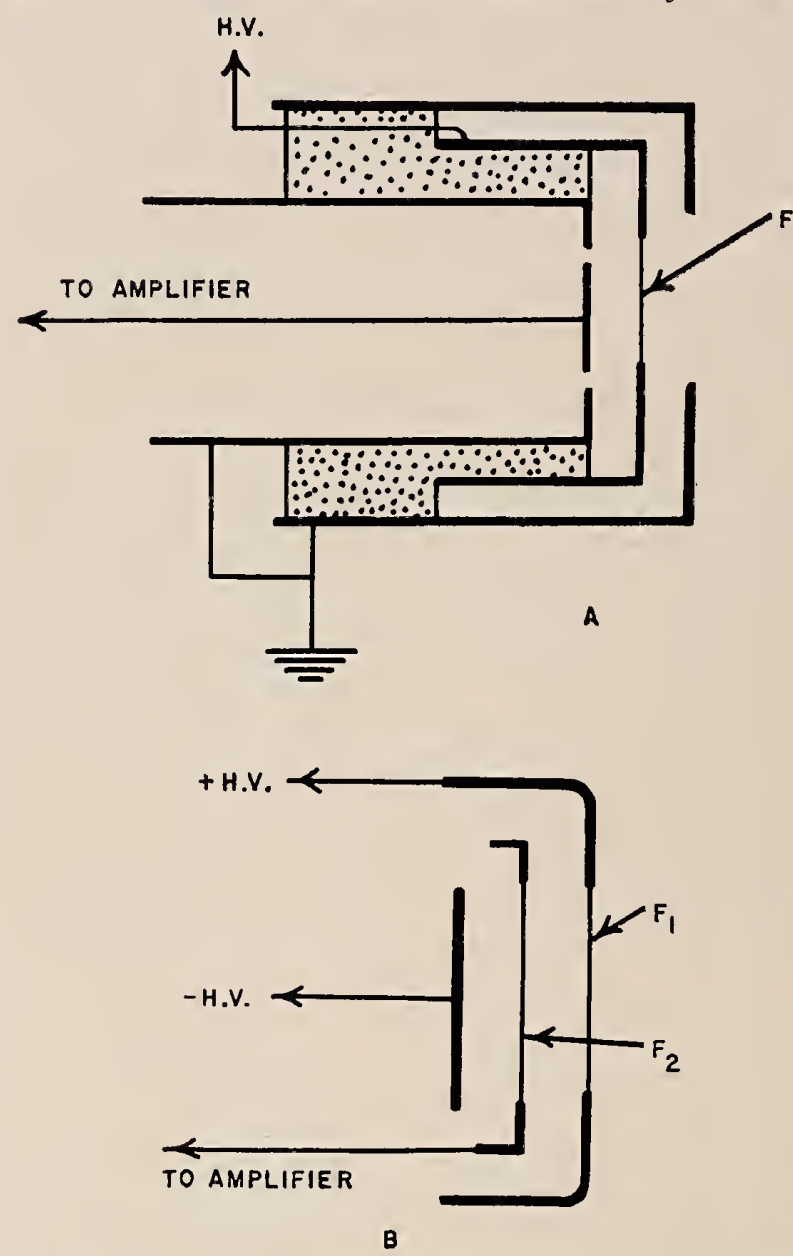

Figure 46. Diagrams of ionization chambers for studying the variation of ionization of alpha particles with range. $A=$ shallow chamber; $F=$ thin metal foil; $H V=$ high voltage;
$B=$ differential chamber; $F_{1}, F_{2}=$ thin metal foils, used as windows.

and Wynn-Williams [34] for the same purpose. These two types of ionization chambers are illustrated in figure 46 . Figure $46, \mathrm{~B}$ represents the shallow chamber. Particles that pass through the grid and penetrate the gas in the shallow volume between the grid and collector plate produce pulses that can be detected by a linear amplifier. There is a minimum height of pulse, which can be differentiated from the background "noise" of the amplifying system, which is represented by a certain minimum depth of penetration of the particle into the 
chamber. This difficulty can be obviated by applying a bias to the amplifier that permits all particles to be recorded which produce pulse heights greater than a definite value somewhat above the minimum that can be detected by the amplifier. The distance of penetration corresponding to this height can be determined and added to the range as measured by the distance from the source to the grid.

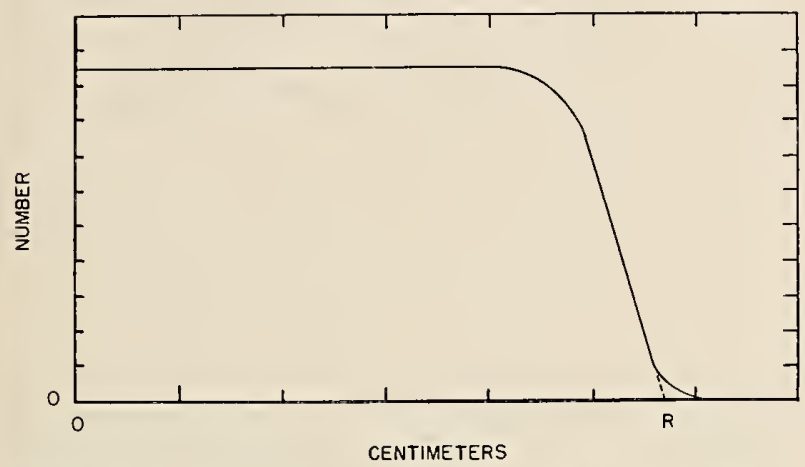

Figure 47. Range-number curve for alpha particles, $R=$ extrapolated range.

Measurements made with a shallow chamber as the source is brought toward the chamber to give a number-distance curve in which the number of particles recorded is plotted against the distance from the source to the chamber. This curve has the general shape shown in figure 47 . This curve shows a practically constant number of particles, which near the end of the range, breaks into a straight line with fairly large negative slope, followed by a curved portion as the number of particles approaches zero. The straight portion of the descending curve when extrapolated to zero at $R$ gives the extrapolated number distance range. This constant is about all that can be determined directly from such an experimental curve.

When the nature of the processes is considered that reduce the energy of the alpha particles and finally deprive them of their ability to produce ionization, it is obvious that the loss of energy occurs in small steps, each representing the result of interaction with atoms of the gas, and this loss of energy will be subject to statistical fluctuations leading to small variations in the ranges of individual particles. This effect has been called straggling by Darwin [35]. These variations are responsible for the shape of the curve shown in figure 47. An analysis by King and Rayton [36] shows that the ranges should be distributed about a mean range, $R_{0}$, and the distribution is given approximately by a Gaussian function

$$
f(x) d x=\frac{1}{\pi^{1 / 2} a} e^{-\frac{\left(R_{0}-x\right)^{2}}{\alpha^{2}}} d x,
$$

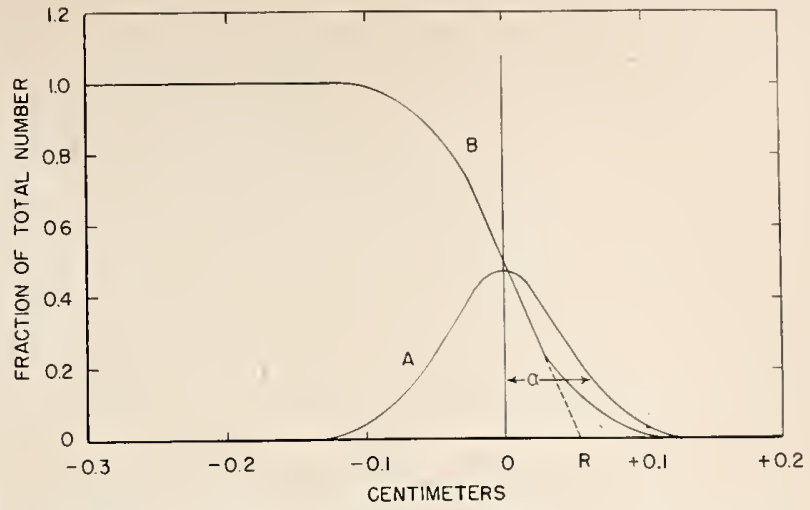

FIGURE 48. Curve showing an analysis of the distribution of ranges of alpha particles.

Curve $B=$ range-number curve near maximum range; $R=$ extra polated range. Curve $A=$ distribution of ranges about the mean range $(x=0), a=$ straggling parameter.

where $f(x) d x$ is the fraction of the total number of particles having a range between $x$ and $x+d x . a / R_{0}$ is the range straggling coefficient; $a$ the range straggling parameter, is the halfwidth of the distribution curve at $1 / e$ of the maximum. This distribution is illustrated in figure 48 where curve $A$ shows the distribution of ranges about the mean range, and $B$ is the corresponding number-distance curve.

\section{Bragg Curve}

The term "specific ionization" is applied to the number of ion pairs per centimeter produced by an alpha particle. By the use of a shallow ionization chamber, this quantity can be determined for very short increments of range throughout the range of a group of alpha particles. This gives the ionization curve corresponding to that of a single alpha particle on which is superimposed the straggling due to variations in range. Such measurements reveal that the specific ionization varies with the velocity of the alpha particle and is in general higher for lower velocities. The typical form of the Bragg curve is shown in figure 49. The extrapolation of the steep front of this curve to zero ionization gives the extrapolated ionization range, which differs from that obtained from the extrapolation of the number-distance curve of figure 47 . In addition, it does not give the mean range, $R_{0}$, except by means of empirically deduced corrections. Therefore, it has become customary to determine the extrapolated range, $R$, from the number-distance curve that leads directly to the mean range by subtracting the quantity $d=\pi^{1 / 2} \alpha$, where $\alpha$ is the straggling coefficient.

The differences between the number-distance and ionization extrapolated ranges is shown in the following values (table 3) quoted from Holloway and Livingston [33], which were measured relative to the ranges of $\mathrm{ThC}^{\prime}, \mathrm{Po}$, and 


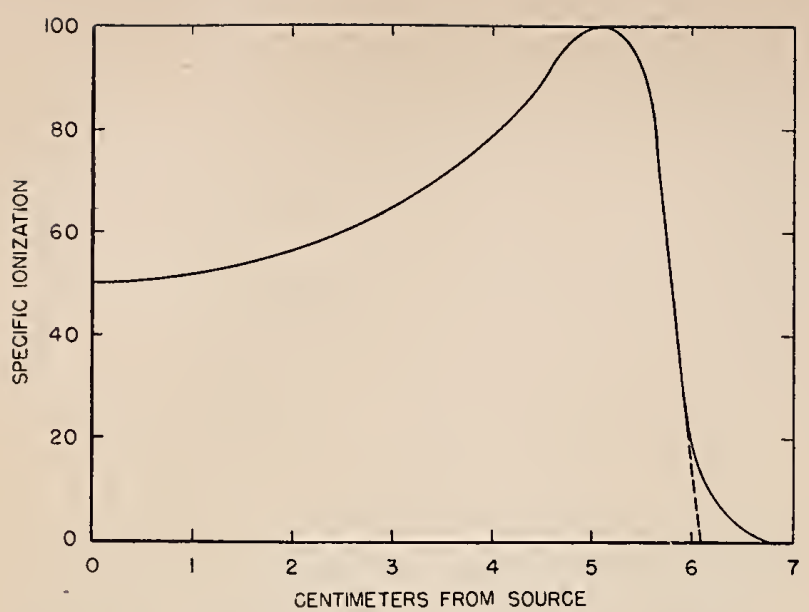

FIGURE 49. The Bragg curve showing the variation of specific ionization with the range of alpha particles.

$\mathrm{RaC}^{\prime}$ alpha particles for which the mean ranges $8.570 \mathrm{~cm}, 3.842 \mathrm{~cm}$, and $6.907 \mathrm{~cm}$ were assumed.

\section{Stopping Power}

This quantity is defined as the reduction in the emergent range produced when a beam of alpha particles traverses a layer of material. A sheet of mica of thickness $1 \mathrm{mg} / \mathrm{cm}^{2}$ will reduce the emergent range of alpha particles incident on it by approximately $1 \mathrm{~cm}$ in air and therefore has a stopping power for alpha particles of $1 \mathrm{~cm}$. The stopping power varies with the velocity of the alpha particle. The relative stopping power of substances of low atomic weight does not show much variation with velocity, but this effect is more pronounced for substances that have a much higher atomic weight than that of the constituents of air.

TABLE 3.-

\begin{tabular}{|c|c|c|c|c|}
\hline \multirow[b]{2}{*}{ Source } & \multirow[b]{2}{*}{ Mean range } & \multirow[b]{2}{*}{$a$} & \multicolumn{2}{|c|}{ Extrapolated ranges } \\
\hline & & & Ionization & $\begin{array}{l}\text { Number- } \\
\text { distance }\end{array}$ \\
\hline AcA. & 6.457 & 0.096 & 6.496 & 6.542 \\
\hline $\mathrm{AcC}$. & 5.429 & .083 & 5.462 & 5.503 \\
\hline Tn.......... & 5.004 & .078 & 5.035 & 5.073 \\
\hline ThC. . & 4.730 & .074 & 4.718 & 4.796 \\
\hline $\mathrm{ThC}^{\prime}$ (long) & 11.580 & .15 & 11.64 & 11.71 \\
\hline
\end{tabular}

The relative atomic stopping power, $S$, for an element of thickness $t$, density $\rho$, and atomic weight $A$, can be computed from the relation $s=\rho_{0} t_{0} A / \rho t A_{0}$, where $\rho_{0}, t_{0}, A_{0}$ refer to a standard substance such as air for which the stopping power is assumed to be unity. On this basis the relative atomic stopping power of aluminum is 1.45 and lead 4.4, whereas hydrogen is 0.22 .

If the stopping power of oxygen is taken as unity, slightly different values are obtained, as shown in table 4. Bragg [37] has shown that the relative atomic stopping power is proportional to $\sqrt{A}$, the atomic weight. Therefore, $S / \sqrt{A}$ should be a constant. The table shows this to be approximately true.

TABLE $4 .-$

\begin{tabular}{|l|c|c||c|c|c|}
\hline Element & $S$ & $S / \sqrt{A}$ & Element & $S$ & $S / \sqrt{A}$ \\
\hline $\mathrm{H}$ & 0.200 & 0.20 & $\mathrm{Fe}$ & 1.96 & 0.26 \\
$\mathrm{Li}$ & .519 & .20 & $\mathrm{Ni}$ & 1.89 & .25 \\
$\mathrm{Be}$ & .750 & .25 & $\mathrm{Cu}$ & 2.00 & .25 \\
$\mathrm{C}$ & .814 & .25 & $\mathrm{Zn}$ & 2.05 & .25 \\
$\mathrm{~N}$ & .939 & .25 & $\mathrm{Ag}$ & 2.74 & .26 \\
$\mathrm{O}$ & 1.000 & .25 & $\mathrm{Cd}$ & 2.75 & .26 \\
$\mathrm{Mg}$ & 1.23 & .25 & $\mathrm{Cd}$ & 2.86 & .26 \\
$\mathrm{Al}$ & 1.27 & .24 & $\mathrm{Pt}$ & 3.64 & .26 \\
$\mathrm{Si}$ & 1.23 & .23 & $\mathrm{Au}$ & 3.73 & .27 \\
$\mathrm{Cl}$ & 1.76 & .29 & $\mathrm{~Pb}$ & 3.86 & .27 \\
\hline
\end{tabular}

\section{Geiger-Nuttall Law}

Geiger and Nuttall [38], from an investigation of the ranges of alpha particles emitted by various naturally radioactive substances, pointed out a connection between range and the halfvalue period of the substances emitting the particles. If the logarithm of the range is plotted against the logarithm of the decay constant, it is found that the points representing alpha particles from substances in the same natural radioactive family fall on a straight line. Thus,

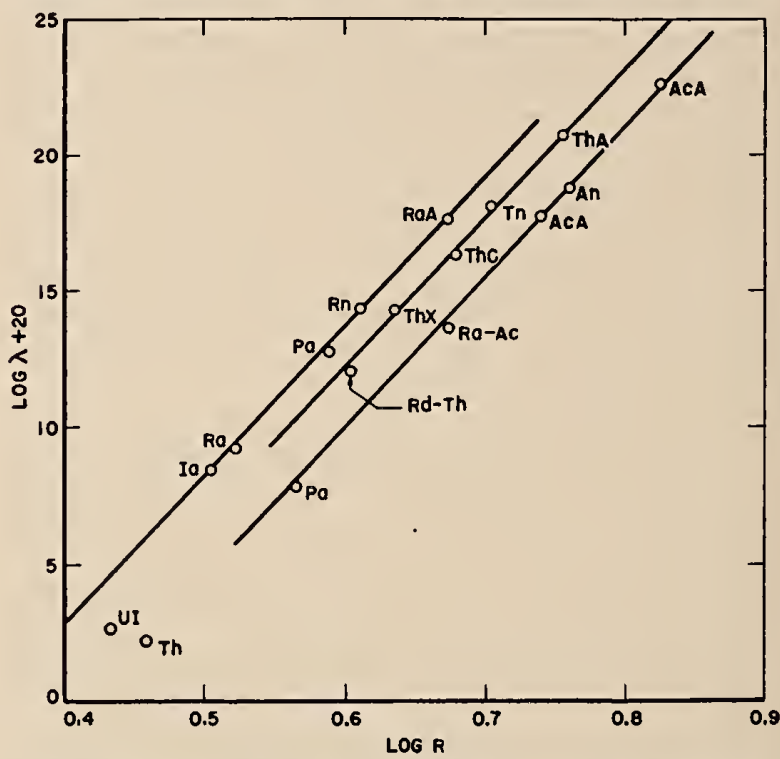

FIgURE 50. Geiger-Nutall curve showing the relation $\log \lambda$ and $\log R$ for various natural alpha-ray emitters.

there is a similar line for each of the three families. These lines are very nearly parallel and can be represented by the relation

$$
\log R=a+b \log \lambda,
$$

where the slope, $b$, is the same for all lines. Figure 50 shows the Geiger-Nuttall plot for the 
uranium, thorium, and actinium families. A few points, those for UI, Th, AcU, AcX and $\mathrm{RaC}^{\prime}$, fall decidedly off the line corresponding to their family.

\section{Scattering of Alpha Particles}

Rutherford [39] was the first to demonstrate that occasionally alpha particles experience angular deviations in their direction of motion. In some instances the angle of deflection is very large. This change in the direction of the particles with little change in kinetic energy is referred to as scattering. The relative number of particles that undergo large changes in direction is small. Geiger and Marsden [40] estimate that about 1 in 8,000 is scattered through an angle greater than $90^{\circ}$. It was pointed out by Rutherford [41] that these large deflections must be the result of a close approach to a single atomic nucleus, and the deflection is the result of the interaction of the electric fields of the nucleus and the alpha particles. For a heavy nucleus, considered to be at rest during the interaction, he pointed out that the path of the alpha particle undergoing a large deflection should be a hyperbola, as shown in figure 51.

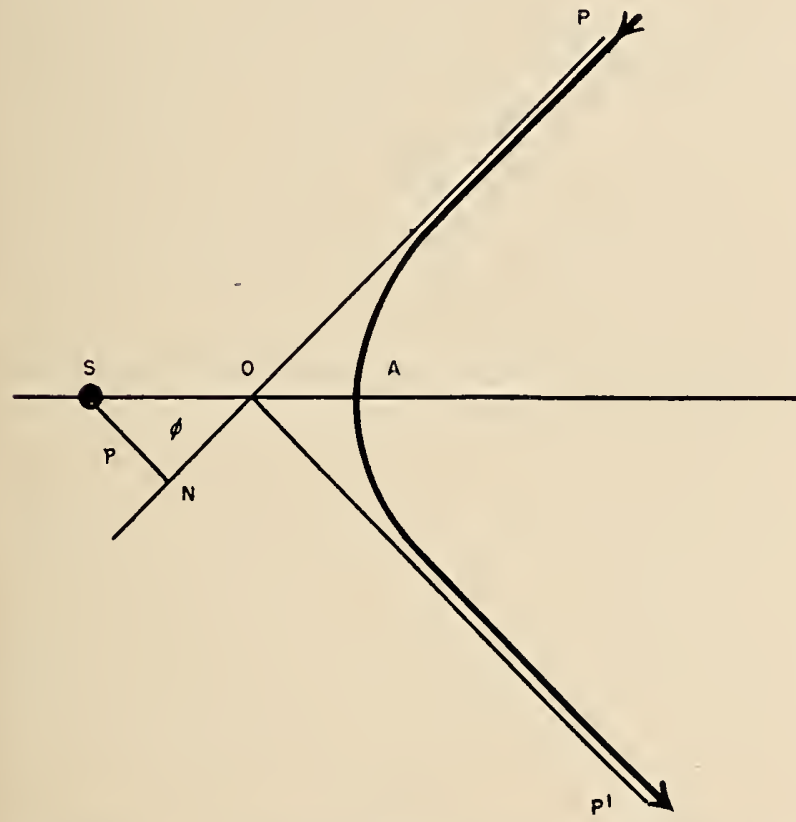

Figure 51. Diagram of the collision of an alpha particle with a nucleus.

$S=$ nucleus; $A=$ point of nearest approach; $P, P^{\prime}=$ path of alpha particle.

In the figure, $S$ is the location of the nucleus, $P O$ the initial direction of the alpha particle, and $O P^{\prime}$ its direction after deflection. $p=S N$ is the perpendicular from the nucleus to the original path of the particle.
Representing the charge, mass, and initial velocity of the alpha particle by $E, M, V$, and its velocity at $A$ as $v$, the conservation of angular momentum requires

$$
p V=\overline{S A} v,
$$

and the conservation of energy requires

$$
1 / 2 M V^{2}=1 / 2 M v^{2}+\frac{Z E}{\overline{S A}},
$$

where $Z$ is equal to the nuclear charge. From this we obtain

$$
v^{2}=V^{2}\left(1-\frac{b}{\overline{S A}}\right)
$$

where $b=2 Z E / M V^{2}=$ distance of closest approach to the nucleus when $p=0$ and the angle of deflection is $180^{\circ}$. From these relations Rutherford deduced that the number of particles, $y$, scattered to a unit area of a detector at an angle $\phi$ to the original direction is given by

$$
y=\frac{Q n t b^{2} \operatorname{cosec}^{4} 1 / 2 \phi}{16 r^{2}}
$$

where $t=$ thickness of scattering layer, $n=$ number of atoms per unit volume, $r=$ distance of detector from the scatterer, and $b$ has the value given above. $Q=$ total number of particles incident on foil. From experiments made by Geiger [42] and by Geiger and Marsden [43], it was found that $y$ is proportional to $\operatorname{cosec}^{4} 1 / 2 \phi$ over the whole range investigated, thus substantiating the concept of the atom accepted today.

\section{Emission of Alpha Particles from the Nucleus}

Experiments on the scattering of alpha particles led to a serious difficulty in explaining how alpha particles escape from the nucleus. For example, using uranium for a scatterer and $\mathrm{ThC}^{\prime}$ alpha particles, no deviation from the Coulomb law of force for the interaction between the nucleus. and particles could be detected. Therefore the uranium nucleus is surrounded by a barrier impenetrable for particles of the energy of $\mathrm{ThC}^{\prime}$ alpha particles, about $8.8 \mathrm{Mev}$. On the other hand, the uranium nucleus emits alpha particles of approximately 4-Mev energy through this same potential barrier.

The application of wave mechanics by Gamow [44] and by Gurney and Condon [45] to this problem eliminates the difficulty in explaining 


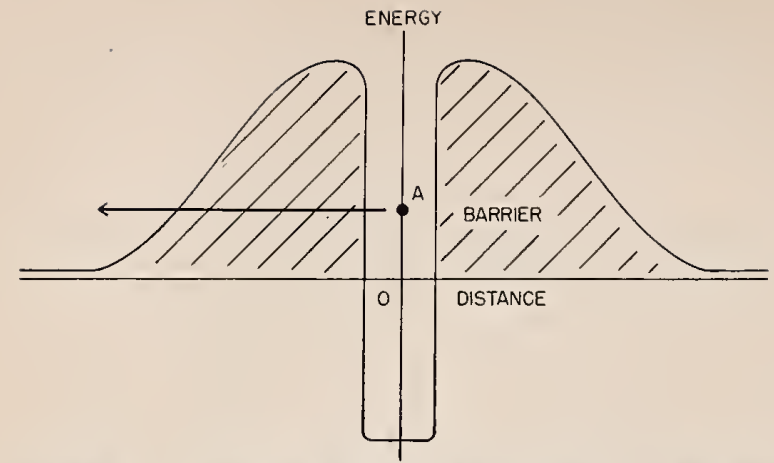

FIgURE 52. Diagram of the potential well of a nucleus. $O=$ center of nucleus: $A=$ alpha particle in nucleus with excitation energy less than the height of the potential barrier. shown shaded.

this phenomenon. Representing the cross section of a nuclear field as shown in figure 52, where distance from the nucleus is plotted on the $x$-axis and the potential energy along the $y$-axis, it is seen that in the central region there is a potential well in which the alpha particles will be retained. However, according to the theory of wave mechanics any particle having a positive excitation energy, as at $A$, has a finite probability of penetrating the potential barrier. The greater the excitation energy, the greater this probability. This means that the decay constant $\lambda$ will be greater for particles of high energy and provides a theoretical basis for the Geiger-Nuttall law. It also explains the fact, at least qualitatively, that the emission of alpha particles in radioactivity decay is confined almost exclusively to nuclei of high atomic number where the potential barriers play a more prominent role.

\section{Beta Particles}

Beta particles are electrons ejected with various energies from radioactive substances. Their identity has been established by deflecting them in electric and magnetic fields. The direction of the deflection revealed that these particles carry a negative charge. The original experiment of this type was performed by J. J. Thomson [46] in which cathode rays of a definite average velocity, $v$, were deflected by electric and magnetic fields. A diagram of the arrangement is shown in figure 53. The magnetic field from the poles $P$ is perpendicular to the plane of the figure. With an electrical field the beam is deflected to some position, $E$, on the fluorescent screen. The magnetic field is arranged to deflect the beam in the opposite sense to some position, $M$. When these two fields are applied simultaneously and adjusted so that no net deflection results

$$
\text { Hev }=E e \text { or } v=\frac{E}{H} \text {. }
$$

With the magnetic field alone $\mathrm{Hev}=m v^{2} / \rho$, or $e / m=v / H_{\rho}$, where $\rho$ is the radius of curvature of the path of the beam in the magnetic field.

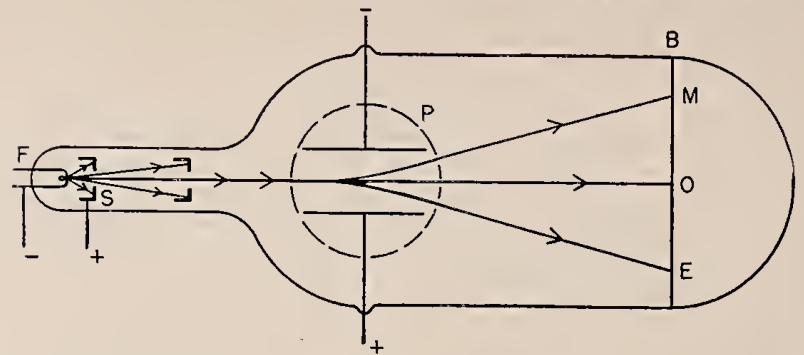

Figure 53. Diagram of apparatus used for original determination of $e / m$ for an electron.

$F=$ filament; $S=$ slit; $P=$ pole of electromagnet; $B=$ fluorescent screen; $M=$ deflection with magnetic field; $E=$ deflection with electric field; $O=$ undeflected position.

$H$ is the intensity of the magnetic field and $E$ that of the electric field in consistent units. Substituting the value of $v$ found from the first equation

$$
e / m=E / H^{2} \rho,
$$

in which all quantities on the right-hand side can be measured. Numerous refinements of similar experiments have led to accurate values for the quantity $\mathrm{e} / \mathrm{m}$ for the electron.

The quantitative identification of the beta particle with the electron was made by Bucherer [47], who determined $\mathrm{e} / \mathrm{m}$ for beta particles from a radioactive source. In this experiment two optically plane circular glass plates silvered on the inner surfaces were mounted with a separation of $0.25 \mathrm{~mm}$. The small radioactive source emitting beta particles was placed between the plates at their center. A uniform electric field was maintained between the silvered glass plates. Simultaneously a uniform magnetic field parallel to the surfaces of the plates was produced by a current in solenoids. To detect the deflected beta rays a photographic film in the form of a cylinder with a diameter of $18 \mathrm{~cm}$ was mounted coaxial with the plates. The particles are all uniformly deflected by the electric field in one direction. The force exerted by the magnetic field, however, depends on the direction as well as the velocity of the particles. Those moving parallel to the magnetic field experience no force, those at an angle $\theta$ with the direction of the magnetic field move toward one plate, if $\theta$ is between $0^{\circ}$ and $180^{\circ}$ and toward the other if it is between $180^{\circ}$ and $360^{\circ}$. If the electric field moves the particles toward the upper plate and the magnetic field for $\theta$ between $180^{\circ}$ and $360^{\circ}$ also moves them toward this plate, no particles will emerge from between the plates for values of $\theta$ between $180^{\circ}$ and $360^{\circ}$. Only those will emerge for which

$$
\text { Hev } \sin \theta=E e \text {, }
$$


which represents the condition that the forces on the electron for the two fields are equal and opposite. After emerging from between the plates, the particles are affected only by the magnetic field. Therefore, the particles move in the arc of a circle of curvature represented by the equation

$$
\text { Hev } \sin \theta=\frac{m v^{2}}{\rho},
$$

$H, E$, and $\rho$ may all be measured experimentally so that the two equations yield both $v$ and $e / m$ for a particular value of $\theta$. Extrapolating the value of $\mathrm{e} / \mathrm{m}$ obtained to zero velocity, a value is obtained for this quantity that agrees with that obtained for electrically accelerated electrons. Also, from this experiment, a verification was obtained for the variation of mass of the electron with velocity in the well-known form

$$
m=\frac{m_{0}}{\left(1-\beta^{2}\right)^{1 / 2}},
$$

where $\beta=v / c, c$ being the velocity of light. This relativistic variation of mass with velocity introduces a modification in the simple expression for the kinetic energy of the electron. For a constant mass the kinetic energy is $1 / 2 m v^{2}$. At relativistic velocities this expression becomes

$$
m_{0} c^{2}\left(\frac{1}{\sqrt{1-\beta^{2}}}-1\right)
$$

The term "beta ray" is now reserved for electrons ejected from the nucleus of an atom. Although they are not considered at present to be constituents of the nucleus as separate entities, they are formed when the nucleus undergoes radioactive disintegration. Therefore, they are to be distinguished from conversion electrons which are also emitted by various radioactive substances but which are "orbital" electrons of the outer atomic structure that have been ejected by nuclear gamma rays. The properties of these two forms of radiation are identical for the same velocities of the particles.

\section{Measurement of Beta Rays}

Beta rays ionize atoms directly by interaction with the outer electrons and therefore can be measured by any of the methods that depend on ionization for their operation. Geiger-Müller counters with thin windows are most commonly used to measure the moderate intensities of beta radiation encountered in usual laboratory experiments. To determine the actual intensity, corrections must be made for absorption in the window. Where the total intensity or number per second from a given source is required, correction must be made also for the relative solid angle subtended by the counter and for those particles emitted initially at some other angle, which are scattered into the counter. A typical beta-ray counter is shown in figure $16, \mathrm{~b}$. The thin mica window, $W$, is at the end of the tube with the central wire terminating in a small glass bead close to the window. The central wire is supported from the opposite end of the counter, as shown in the diagram. The body of this counter is usualiy made of copper. Such a counter has a very high efficiency of response for all beta particles that penetrate the mica window.

Geiger-Müller counters of the form shown in figure 16 , a are also useful in comparative measurements of beta radiation of energy above 0.35 Mev.

The cylindrical tube, $T$, which also serves as the envelope for the counter, is sufficiently thin to admit beta rays. Since the metal tube, usually of aluminum, must withstand evacuation in the process of filling, the wall thickness must be at least $0.1 \mathrm{~mm}$ for a tube with a diameter of $1.5 \mathrm{~cm}$ and $10 \mathrm{~cm}$ in length. This thickness represents $27 \mathrm{mg} / \mathrm{cm}^{2}$, which absorbs all particles with energies less than about $0.35 \mathrm{Mev}$. Similar counter tubes with thin silvered glass walls have been made.

\section{Measurement of Beta Rays with End Window Counters}

To obtain reliable comparative measurements with thin mica window counters, it is necessary that absorption of beta rays in the source be either negligible or the same for each source. The amount of material in the source can be as much as 10 to $20 \mathrm{mg}$ if the maximum energy of the beta rays is of the order of $1 \mathrm{Mev}$, whereas not more than 1 or $2 \mathrm{mg}$ can be tolerated for radiation of maximum energy of the order of $0.1 \mathrm{Mev}$, unless corrections are made for the absorption in the source. Also, the active material must be uniformly distributed in the source. Since the solid angle decreases for positions of the source off the axis of the counter, it is necessary to center each source accurately with respect to the axis. For the same reason the diameter of the source should be kept as small as practicable in comparison with the diameter of the counter window.

The over-all efficiency of the beta-ray counter may be defined as the ratio of the observed counting rate to the rate of disintegration of the sample when the sample consists of a single isotope with a simple beta-ray spectrum. This ratio depends on the solid angle, but for a given 
solid angle it will vary with the energy of the beta rays for different isotopes. This occurs because the observed counting rate also depends on absorption in the source, absorption in the air between the source and the window, and absorption in the window. The counting rate is also affected by scattering of beta particles into the counter, which were emitted in some other direction, usually called "back scattering".

Self-absorption may be determined for a given type of source by preparing deposits of the same diameter and different amounts of inert material and determining the number of counts per milligram in a standard position with respect to the counter. This will give a smooth curve that can be extrapolated to zero thickness. From the value at zero thickness the value of the self-absorption in percentage of the zerothickness value can be computed for the various thicknesses in milligrams per cubic centimeters of the samples used. Similar results are obtained by using increasing amounts of material with a constant specific activity.

External absorption may be treated in much the same way. The absorption curve may be determined for the radiation from a given radioisotope by interposing various absorbers of known thickness. To each thickness in milligrams per square centimeter must be added the corresponding value for the mica window and the intervening air. This curve, plotted on semi$\log$ paper, will give approximately a straight line that can be extrapolated to zero thickness. This counting rate permits a computation of the percentage absorption for the various absorbers used and for the window and intervening air. This value must be determined for each radiation of different maximum energy.

Back-scattering can be reduced by mounting samples on very thin supports of low atomic number. A value for zero thickness of support may be obtained by increasing the thickness of the support in definite steps and observing the counting rate at each step. In many cases it may be preferable to use a support thick enough that the back-scattering has reached the saturation value for all samples. Relative values are not affected by this procedure.

\section{Beta-Ray Standards}

Much of the work required in determining the over-all efficiency of a beta-ray counter may be reduced by the use of a beta-ray standard. Such a standard consists of a preparation for which the disintegration rate, the maximum energy of the beta rays, and the thickness of the support are known. In practice it is convenient to prepare such standards with the support thick enough to produce saturation backscattering. This requires that all preparations compared with this standard be mounted in a similar manner. The over-all efficiency is given at once by mounting the standard in a selected position relative to the counter and determining the counting rate. The ratio between this rate and the disintegration rate for the standard is the over-all efficiency. Beta-ray, standards must emit radiation of approximately the same maximum energy as that of the radioisotope measured by the calibrated counter. Such standards are available from this Bureau.

When gamma radiation is also present, it may be necessary to correct for its effect on the beta-ray counter. In most cases the effect of the gamma radiation will be of the order of 1 or 2 percent due to the low efficiency of the counter for gamma rays. The effect can be determined by introducing lead absorbers and obtaining an absorption curve that can be extrapolated back to zero thickness and deducing this extrapolated value from the observed rates. In other cases where comparative measurements with the same isotope are made, the presence of a small gamma-ray effect produces no error.

\section{Electroscopes}

The Lauritsen electroscope is useful in making comparative measurements of beta rays. Very thin aluminum windows may be used to reduce the loss of particles by absorption. When calibrated with standards, they will yield absolute values. Since the time of observation is limited for each reading, the average of a number of readings must be taken to obtain a sta-

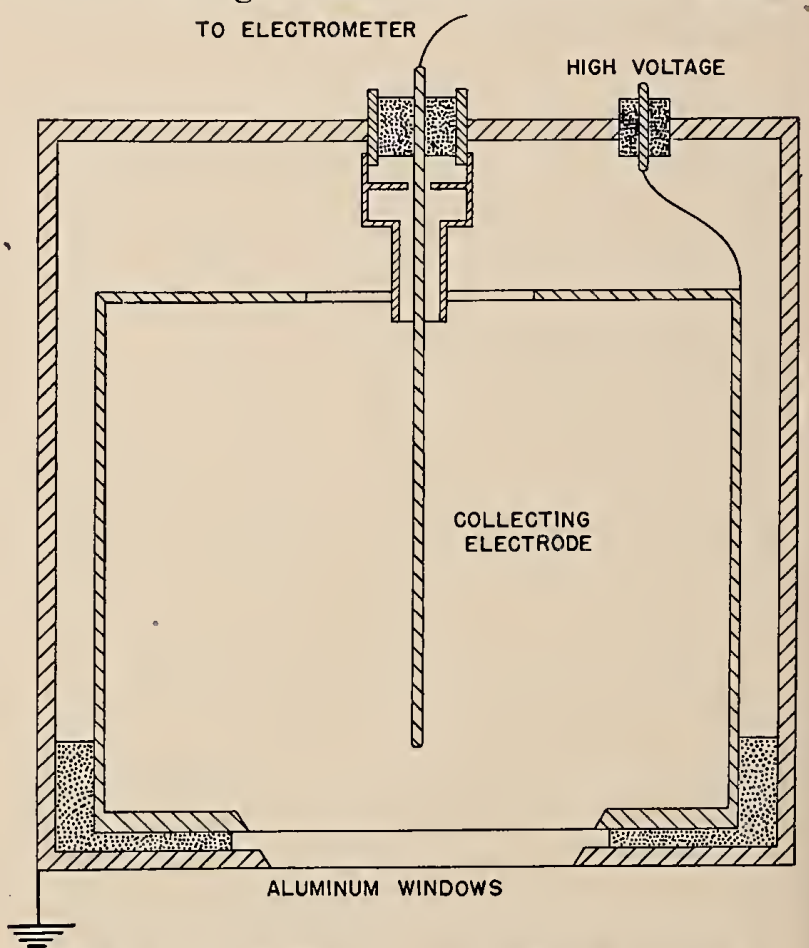

FIgURE 54. Cross section of an ionization chamber for measuring beta rays. 
tistically accurate result. The Lauritsen electroscope is less sensitive than Geiger-Müller counters, and its indications are linear over a limited range of intensities.

\section{Ionization Chambers}

For many purposes, particularly for sources of a considerable range of intensity, an ionization chamber as sketched in figure 54, may be used. The collecting electrode is connected to an electrometer or a vacuum tube circuit using an FP-54 type of vacuum tube. By incorporating a Townsend compensating condenser or a potentiometer, a null deflection method may be used. Such an arrangement has a large range of linear response permitting very strong and relatively weak sources to be measured under identical conditions.

\section{Beta-Ray Spectra}

We have noted that beta rays consist of electrons of various velocities and therefore can be deflected in a magnetic field. Those travelling with the same velocity will travel along a circular path of the same radius. By this means it has been possible to separate beta particles and record them as beta-ray spectra, which reveal the intensity of the beta radiation for various values of $H_{\rho}$ proportional to the momentum of the particles. One method of studying these spectra is by semicircular focussing represented in figure 55 . In the figure the magnetic field is assumed perpendicular to the plane of the figure, and $S$ is a source of beta radiation of various velocities. Semi-circles are shown for two different velocities. Above the source is a relatively wide slit through which the particles emerge, travelling in the circular paths defined by their velocities and the strength of the magnetic field. It is seen that all particles emitted from a small source over the full angle subtended by the slit at the source are brought together in a sort of reversed image of the source at the opposite end of the diameter of the circle in which they are travelling. This focussing property of the circular path produces relatively sharp lines for homogeneous velocities. Part of all such spectra consists of a continuous distribution of velocities up to a definite upper limit in which no homogeneous groups or lines appear. To record these spectra, by using the semicircular method of focussing, a photographic plate, $P P$, is mounted in the plane of the slit. Then assuming that the source is symmetrically located, with respect to the slit, the radius of curvature, $\rho$, corresponding to the various lines is given by $4 \rho^{2}=a^{2}+b^{2}$, where $a$ is the distance from the source to the center of the slit, and $b$ is the distance from the high velocity edge of the photographic image of the

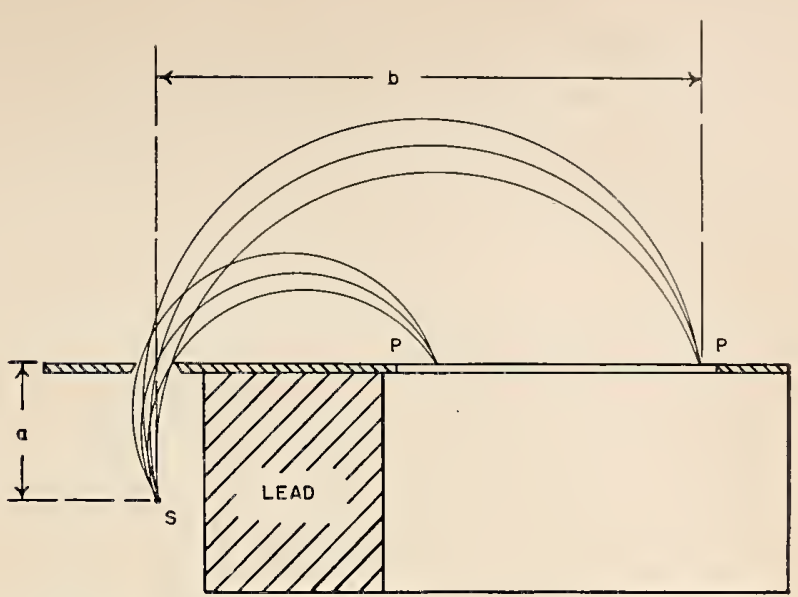

Figure 55. Diagram of $180^{\circ}$ semicircular focussing beta-ray spectrograph.

$$
S=\text { source; } P, P=\text { photographic plate. }
$$

source to the center of the slit. The momentum of the particles is $H_{\rho} e$, where $H$ is the field, and $e$ the electronic charge in emu. However $H_{\rho}$ is directly determined experimentally and represents

$$
H_{\rho}=\frac{m_{0} c}{e} \frac{\beta}{\sqrt{-1 \beta^{2}}}
$$

from which the energy, $E$, in ergs can be calculated by the relation

$$
E=m_{0} c^{2}\left(\frac{1}{\sqrt{1-\beta^{2}}}-1\right),
$$

in which $m_{0}$ is the rest mass of the electron. The energy of beta particles is commonly expressed, not in ergs, but in electron volts, which represents the potential difference in volts through which the electron must be accelerated to obtain a given energy. One erg represents $6.24 \times$ $10^{5}$ Mev.

Beta-ray spectra are made up of two types of particles differentiated according to their origin. The term beta particle has come to be reserved for the primary, or disintegration, particle emitted from the nucleus causing a change in the nuclear charge. These particles are emitted in the form of continuous spectra with all energies present up to a definite maximum. In addition to these primary rays, among the heavier elements in particular, particles are emitted in sharply defined groups, sometimes called the beta-ray line spectra. These particles are conversion electrons originating from the internal conversions of nuclear gamma rays. Since gamma rays are the result of nuclear transitions from one level to another, they are emitted with sharply defined energies, that accounts for the sharpness 
of the lines in the conversion electron spectra. If $E_{1}$ represents the energy of the ejected conversion electron, $E_{\gamma}$ the energy of the gamma ray, and the $E_{K}$ the binding energy of orbital electrons in the $K$-shell, these quantities are related according to the equation

$$
E_{1}=E_{\gamma}-E_{K}
$$

Since conversion may occur for other than the $K$-level, several similar equations may be set up for a single gamma ray, as $E_{2}=E_{\gamma}-E_{L}$ and $E_{3}=E_{\gamma}-E_{M}$, where $E_{L}$ and $E_{M}$ represent the binding energies of $L$ and $M$ shell electrons. The observation of conversion electrons with energies corresponding to $E_{1}, E_{2}$, and $E_{3}$ forms one of the strongest experimental proofs of the correctness of the conversion hypothesis. The semicircular focussing beta-ray spectrograph has been used mainly for the study of line spec-

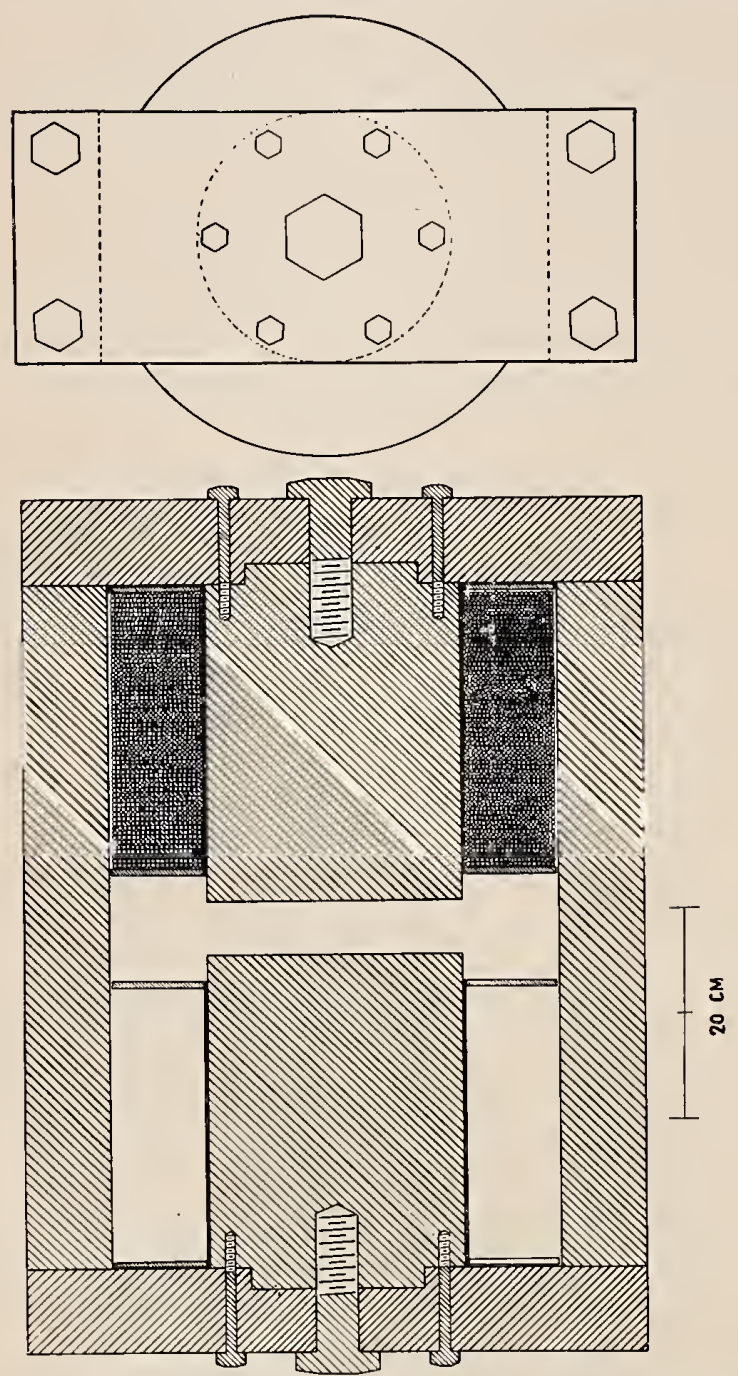

FIGURE 56. Cross section of an electromagnet for $180^{\circ}$ beta-ray spectrograph. tra of conversion electrons by using a photographic plate for recording. Continuous spectra do not lend themselves to photographic recording, since it is difficult to distinguish the maximum end point.

The use of a Geiger-Müller counter to detect the beta particles is required to convert this instrument for the study of continuous spectra. Since the counter is operated in a fixed position the magnetic field must be varied in small accurate steps to obtain a spectrum. Magnets with iron cores, either electromagnets or semipermanent magnets, have been used with the semicircular spectrograph, but it is difficult to change the magnetic field with such magnets in the way desired. On the other hand, these magnets are entirely satisfactory for photographic recording of line spectra.

\section{Magnets for Semicircular Beta-Ray Spectrographs}

The simplest magnet for this purpose consists of an electromagnet of yoke design using low-carbon steel for the yoke and core. Figure 56 is a cross section of a magnet of this type. An air gap of approximately $5 \mathrm{~cm}$ is adequate

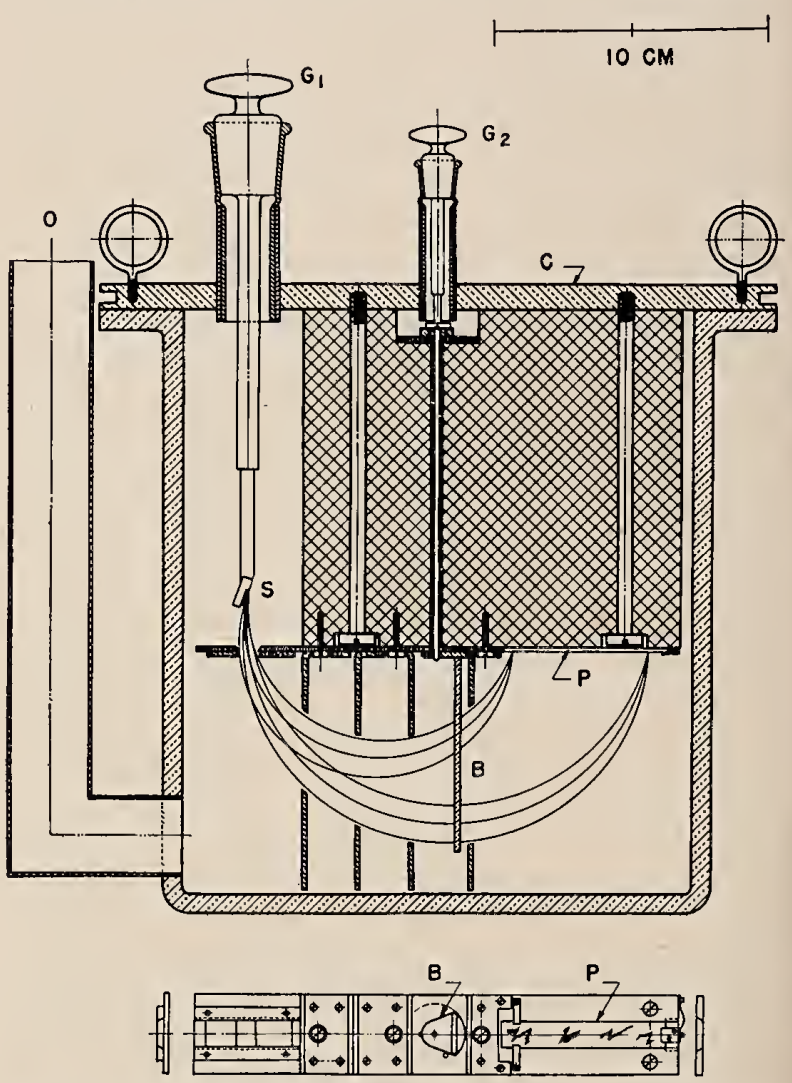

Figure 57. Cross section of typical $180^{\circ}$ beta-ray spectrograph.

$O=$ pump connection; $G_{1}=$ source holder; $S=$ source; $G_{2}=$ shutter holder; $B=$ shutter; $C=$ cover; $P=$ photographic plate. 
for the spectrometer. A typical spectrograph is shown in section in figure 57 . The magnetic field is adjusted by selecting the value of the current desired and reversing this current several times. In this way a definite point is selected on the hysteresis curve of the iron that is quite accurately reproducible. The desired current is selected from a curve showing the magnetic field for various values of the current obtained by the same method of reversing the current.

\section{Magnetic Electron Lens Spectrometer}

Klemperer [48] proposed in 1935 the use of a short magnetic lens to focus beta rays. The principles involved are described by Deutsch, Elliott, and Evans [49]. Figure 58 shows a diagram used by Bouwers [50] to explain the action of a thin magnetic lens on the trajectory of a beta particle. If we consider a particle entering the magnetic coil from the left with velocity $(v)$ parallel to the axis and a small radial velocity $v \sin a$, the electron is first deflected sidewise by the Lorentz force due to the radial component of the magnetic field at the entrance to the coil, and then moves along a helical line with slightly varying radius. The inward movement is produced by the force due to the axial magnetic field and the lateral velocity. The lateral speed decreases when the electron leaves the coil, since the radial component of the magnetic field is reversed, and the axial speed of the electron has been maintained. The rotation has ceased when the electron has left the magnetic field. It is under these conditions that the beta particle will intersect the axis on its continued path. It is seen that the radial component of the short magnetic coil is essential for the formation of an electron image.

The electron lens yields an image of high intensity, since it focusses all electrons of a given velocity in a fairly large solid angle in an image of the source at the focal point. It also possesses the advantage that no iron need be included in the coil, so that the magnetic field is always directly proportional to the current in the coil.

Figure 59 shows a section of a spectrometer adapted for convenient use. The cylindrical symmetry leads to the use of annular diaphragms, that defiue circular slits, which in turn determine the angle, $X$, between the initial direction of the particle and the axis of the lens. A central lead baffle is introduced to shield the counter from gamma radiation from the source. One of the annular baffles is adjustable from the outside through a Wilson seal so that the resolution of the instrument can be varied. Provision is made for introduction of the source through a Wilson seal and a gate valve with rubber gasket. This permits the source to be changed while maintaining the vacuum in the spectrometer chamber. Thus the window of the Geiger-Müller counter is only required to withstand a pressure difference equal to the pressure inside the counter, usually 8 to $10 \mathrm{~cm} \mathrm{Hg}$.

Although such an instrument could be calibrated from its dimensions, it is simpler to use the conversion electrons from natural radioactive sources for which the values of $H_{\rho}$ have been accurately determined. The linearity of the calibration curve makes such a calibration relatively simple, since two or three points definitely determine the complete calibration. Figure 60 shows a typical conversion electron spectrum obtained with this instrument. The peaks are produced by the electrons released from a uranium radiator irradiated by gamma rays from $\mathrm{Sc}_{46}$.

\section{Continuous Beta-Ray Spectra}

The continuous beta-ray spectra have been identified as the disintegration beta rays emitted by the nucleus. The form of these spectra, as shown in figure 61 , indicates that these beta rays are ejected with all velocities from zero to a definite maximum, with the greatest number having an energy of approximately onethird of the maximum value. This type of spectrum is very difficult to reconcile with a nuclear structure in sharply quantized energy states. Consequently, this continuous spectrum was at first interpreted as representing the resultant of multiple absorption and scattering processes suffered by initially monoenergetic radiation. This view was forced into the discard by calorimetric measurements by Ellis and Wooster [51]. They measured the total energy of the

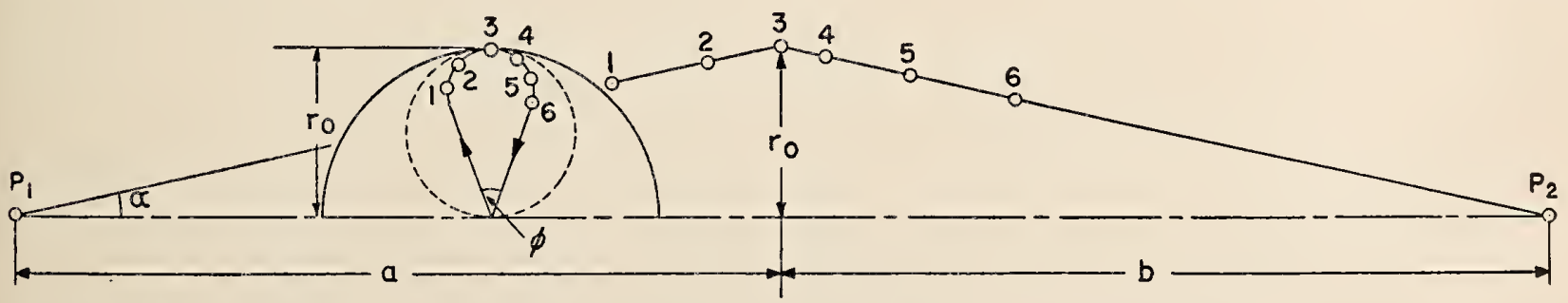

Figure 58. Diagram of the trajectory of an electron in a thin magnetic lens spectrometer. $P_{1}=$ source; $P_{2}=$ image. The diagram in dotted circle shows an end view of the trajectory. $a=$ Source to center of lens distance; $b=$ center of lens to image distance. 


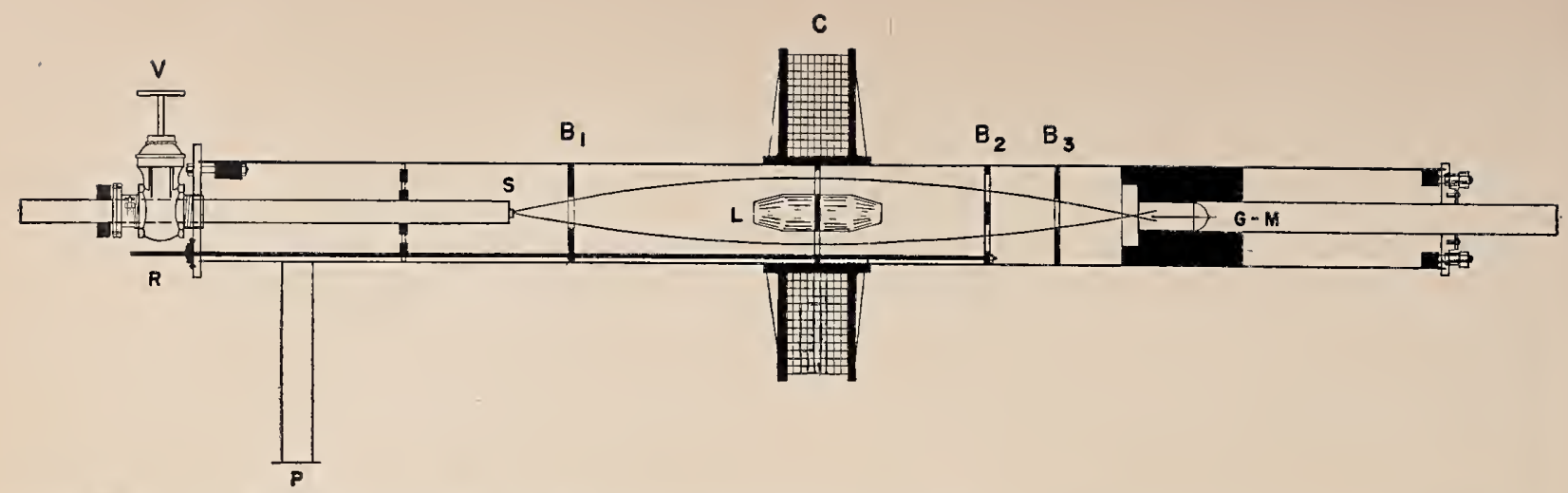

Figure 59. Cross section of thin magnetic lens electron spectrometer.

$C=$ lens coil; $B_{1}, B_{3}=$ fixed baffles; $B=$ adjustable baffle moved by means of rod, $R ; L=$ lead baffle: $P=$ pump connections; $V=$ gate valve for introducing source, $S$, without disturbing vacuum; $G M=$ Greiger-Müller counter.

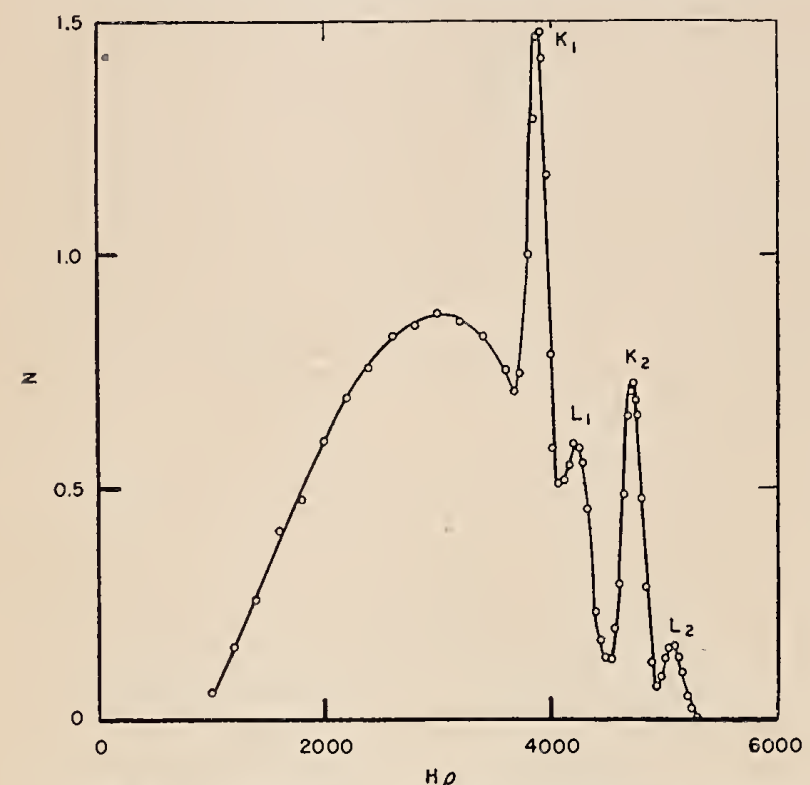

FIGURE 60. Spectrum of secondary conversion electrons ejected from a uranium radiation by gamma rays of $\mathrm{Sc}^{46}$.

The conversion spectrum is superposed on a background of Compton electrons scattered from source holder.

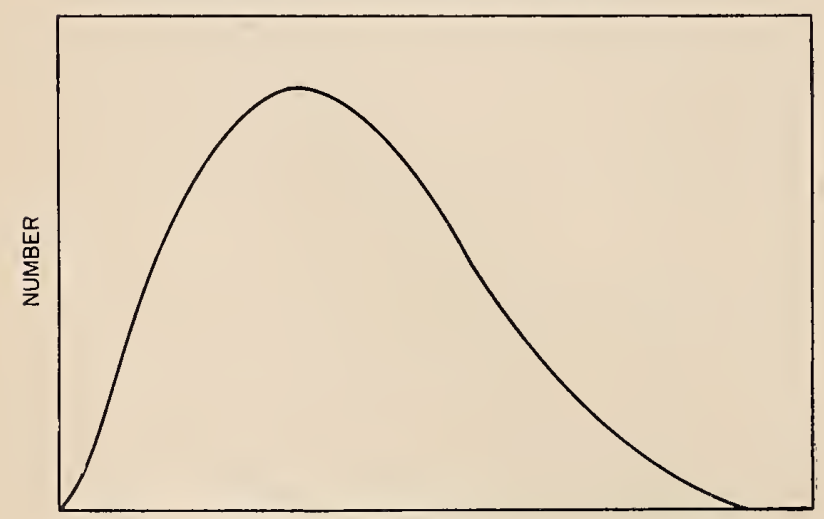

ENERGY

FIGURE 61. Typical primary beta-ray spectrum showing a continuous distribution of particles of all energies up to the maximum. beta rays from $\mathrm{RaE}$ by determining the heat developed by the absorption of these beta rays in a micro-calorimeter. This experiment proved that the thermal energy agreed with that determined from the actual measured spectrum. If these particles had all been emitted from the nucleus with the maximum energy, the calorimetric measurements should have yielded a value nearly three times as great as was observed. Therefore this continuous spectrum is definitely a characteristic of the disintegrating nucleus.

However, the maximum energies of continuous beta-ray spectra play an important role in the investigation of regularities between the disintegration energies and the decay constants, the first investigated by Sargent [52], who plotted the logarithm of the maximum energy against the logarithm of the decay constant. His results are shown in figure 62 . For most isotopes the points fall on two parallel lines separated by a factor of 100 in the decay constant. This has been interpreted to be the

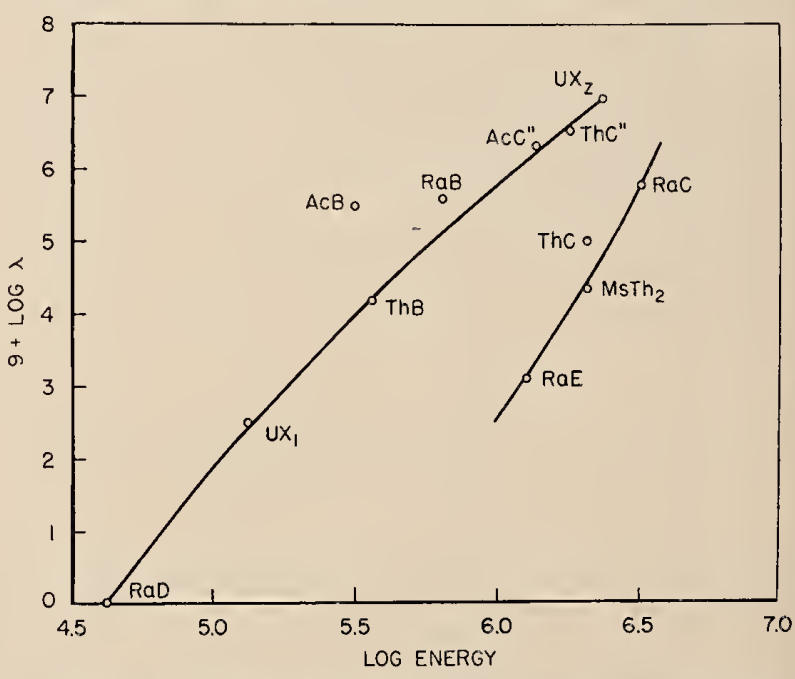

FIGURE 62. Graph showing relations between $\log \lambda$ and log of maximum energy for some natural beta-ray emitters (Sargent curves). 
result of a selection rule for beta disintegration in which those transitions involving a small change in the total angular momentum are permitted and all others forbidden. Actually it can be shown that forbidden transitions theoretically occur with a probability of the order of $1 / 100$ of that for those that are permitted according to theory. The Sargent rule, determined empirically, confirms this view. An important point is that in plotting the Sargent diagram the maximum energies of the various beta-ray spectra are used.

An even more convincing argument indicating the fundamental importance of the maximum energy of the continuous beta-ray spectra is offered by a consideration of the balance of energy in radioactive disintegrations. It is found here that the energy lost per beta-ray disintegration by the decaying nucleus is just that of the upper limit of the beta-ray spectrum. This is demonstrated by the frequently cited example of the disintegration of ThC, which proceeds by two routes to stable $\mathrm{ThD}$, as illustrated in figure 63. As shown, ThC may emit an alpha particle of $6.05 \mathrm{Mev}$ and an insignifi-

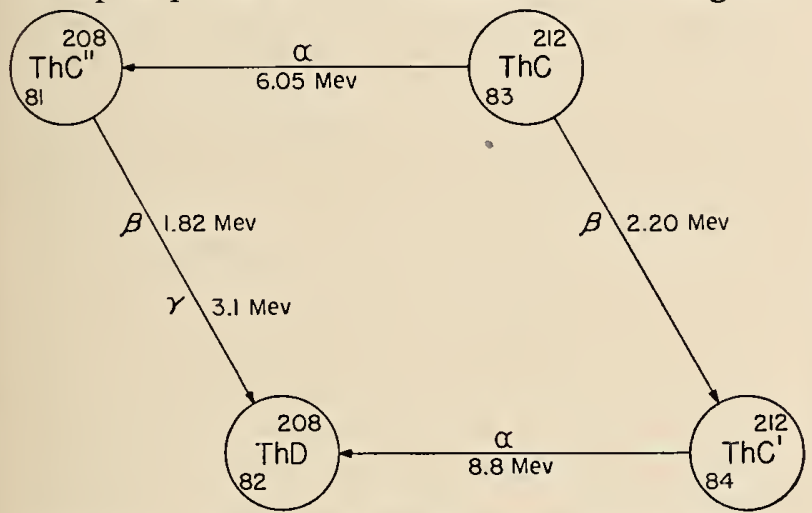

FIGURE 63. Diagram showing disintegration energies in the branching decay of $\mathrm{ThC}$.

cant amount of gamma radiation to form $\mathrm{ThC}^{\prime \prime}$, or it may emit a beta-ray spectrum with an upper limit of $2.20 \mathrm{Mev}$ to become $\mathrm{ThC}^{\prime}$. The ThC" emits a beta-ray spectrum with an upper limit of $1.82 \mathrm{Mev}$ plus two gamma rays, one of 2.62 , the other $0.58 \mathrm{Mev}$, in going over to ThD, whereas ThC' emits an alpha particle of 8.8 $\mathrm{Mev}$ to transform into ThD. If we add the energies over the two paths we find $\mathrm{ThC} \rightarrow \mathrm{ThC}^{\prime \prime}$ $\rightarrow \mathrm{ThD}=6.05+1.82+2.62+0.58=11.07 \quad \mathrm{M} \mathrm{e} \mathrm{v}$, and $\mathrm{ThC} \rightarrow \mathrm{ThC}^{\prime} \rightarrow \mathrm{ThD}=2.20+8.8=11.0 \quad \mathrm{M}$ e v . Therefore we must conclude that each disintegration beta ray carried energy corresponding to the upper limit of its spectrum and not the energy corresponding to the continuous spectral distribution.

\section{Neutrino}

The problem posed by the existence of a continuous beta-ray spectrum leads to one of two general alternatives, either (1) the law of conservation of energy must be abandoned or (2) the disintegrating nuclei must lose energy by means of some hitherto undetectable radiation, so that for each beta ray the sum of its energy plus that of the unknown radiation emitted simultaneously equals the energy of the upper limit of the spectrum. The second alternative has appeared preferable. It was proposed by Pauli that the problem could be explained by introducing the idea of a hypothetical particle, which he called the neutrino, having no electric charge and a small mass compared with that of an electron. The absence of electric charge would make it impossible to detect the neutrino by direct ionization, and the small mass would greatly reduce the chances of communicating energy by impact except in very thick layers of matter, also a very difficult process to detect. Furthermore, most of the neutrinos must have relatively high velocities to carry the energy ascribed to them.

\section{Fermi Theory of Beta-Ray Decay}

Fermi [53] has developed a tentative theory of beta emission on the basis of the simplest hypothesis concerning the interaction energy between neutrons and protons, as well as between electrons and neutrinos, resulting in the transformation of neutrons into protons and the liberation of electrons and neutrinos. Also, the view proposed by Heisenber'g [54]) that the nucleus consists only of neutrons and protons is accepted in this theory, where the neutron and the proton are regarded as two quantum states of the heavy particle. In this picture an electron and neutrino appear for each transition from a neutron to a proton and disappear for the reverse transition. On application of the Dirac, Jordan and Klein [55] method of "second quantization" the probability $P(\eta) d_{\eta}$ of transition into an electron state with a momentum interval of $m c d_{\eta}$ is computed, where $\eta$ is the momentum of the particle in units of $m c\left(\eta=H_{\rho} / 1704\right)$. An approximate value is given by

$$
\begin{aligned}
& P(\eta) d \eta=1.75 \times 10^{95} g^{2}\left|j v_{m}^{*} u_{n} d \tau\right|^{2} \\
& \quad\left(\eta+0.355 \eta^{2}\right) \quad\left(\sqrt{1+\eta_{0}^{2}}-\sqrt{\left.1+\eta^{2}\right)^{2}} .\right.
\end{aligned}
$$

In this expression $\eta_{0}$ is the maximum momentum of the emitted beta ray and $j v_{m}^{*} u_{n} d_{\tau}$ is an integral that requires information regarding the eigenfunctions of the proton and neutrons in the nucleus. For permitted transitions, it must have a value near unity. The constant $g$ may be very roughly estimated from experimental data regarding half-periods of beta-ray transitions with simplifying assumptions. It comes out

$$
g=4 \times 10^{-50} \mathrm{~cm}^{3} \mathrm{erg} .
$$


Kurie, Richardson, and Paxton [56] have suggested that by dividing the expression for $P(\eta) d_{\eta}$ by the corresponding one for the halflif $\epsilon$, as given by Fermi, we obtain for the number' of particles $N$ between $m c \eta$ and $m c$ $(\eta \cdot \triangle \eta)$

$$
N \triangle \eta=K f(Z, \eta) \quad\left[C-\left(1+\eta^{2}\right)^{1 / 2}\right]^{2} \quad(\triangle) \eta,
$$

where $K$ is a function of $Z=$ atomic number, $r=$ half-period, and $\eta_{0}=$ momentum at the upper limit of the spectrum; and $C=\left(1+\eta_{0}^{2}\right)^{1 / 2}=$ $E_{0}+1$, where $E_{0}$ is the energy corresponding to $\eta_{0}$. For all light elements up to about copper $(Z=29)$

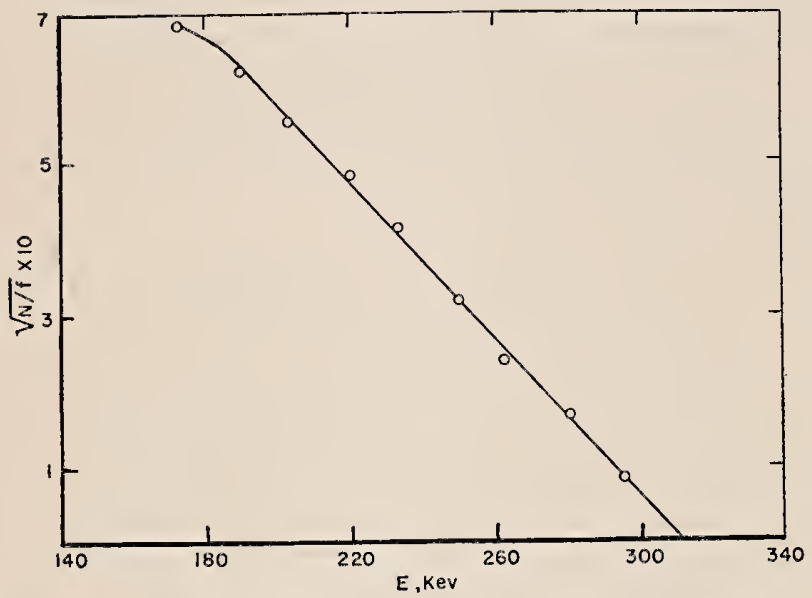

FiguRE 64. Fermi plot for the beta-ray spectrum of $\mathrm{Co}^{60}$.

$$
f(Z, \eta)=\eta^{2} \frac{2 \pi y}{1-e^{-2} \pi y},
$$

where

$$
y=\frac{Z\left(1+\eta^{2}\right)^{1 / 2}}{137 \eta}
$$

Therefore from eq 80 , it is seen that

$$
(N / f)^{1 / 2} a\left(E_{0}-E\right) \text {, }
$$

and by plotting $(N / f)^{1 / 2}$ against $E$, the Fermi theory gives a straight line with the energy of the upper limit, $E_{0}$, given by the intercept on the $E$ axis. As more reliable data on beta-ray spectra have become available, it has been found that the Fermi theory yields the expected straight line except for lower energies where experimental errors are numerous due to scattering effects. A typical Fermi plot for $\mathrm{Co}^{60}$ is shown in figure 64 .

\section{Absorption of Beta Particles}

The small mass of the electron, compared with that of an alpha particle, is responsible for the much greater penetrating power of beta particles of the same energy. In passing through matter the number of beta particles is reduced by gradual loss of energy until they are absorbed. Hence those that finally emerge through a given thickness of matter have an average energy lower than that of the incident particles. The beta radiation emitted by radioactive substances is, as we have seen, distributed over a considerable range of energies. When conversion electrons are present their spectrum, consisting of well-defined lines, is superimposed on this continuous distribution. A number of artificially radioactive isotopes that decay by isomeric transitions emit electron spectra composed predominantly of conversion electrons from strongly converted gamma rays. However, the absorption and scattering processes occurring as these particles traverse an absorber result in a wide distribution of ranges.

As the beta particles pass through matter they give up energy by ionizing the atoms encountered. At energies of the order of $2 \mathrm{Mev}$, a beta particle produces about 25 ion pairs per centimeter of air at atmospheric pressure, whereas particles of about $0.05 \mathrm{Mev}$ produce 200 ions per centimeter. The average energy lost per pair of primary ions formed is around $35 \mathrm{ev}$, indicating that a considerable amount of secondary ionization is produced along with excited atoms.

\section{Scattering of Beta Porticles}

As beta particles pass through matter, they occasionally enter the fields of the nuclei of the atoms of the absorber and are deflected with little change in energy. In this way they may be scattered through large angles up to $150^{\circ}$. Scattering near the maximum angle is called back-scattering, since the particles are moving in a general direction opposite to that in which they enter the absorber. The average angle of scattering is proportional to the thickness of the scattering layer, whereas the number scattered at a particular angle is proportional to $Z^{2}$, the square of the atomic number of the atoms of which the scattering layer is composed.

\section{Range of Beta Particles}

Although the ranges of beta particles are much less sharply defined than in the case of alpha particles, the maximum range can be fairly well determined. The maximum range is that thickness of material, usually measured in grams per square centimeter, for which beta rays of a given energy are just completely absorbed. Aluminum is a convenient absorber for making such measurements of range, and it is 
customary to express ranges in grams per square centimeter of aluminum.

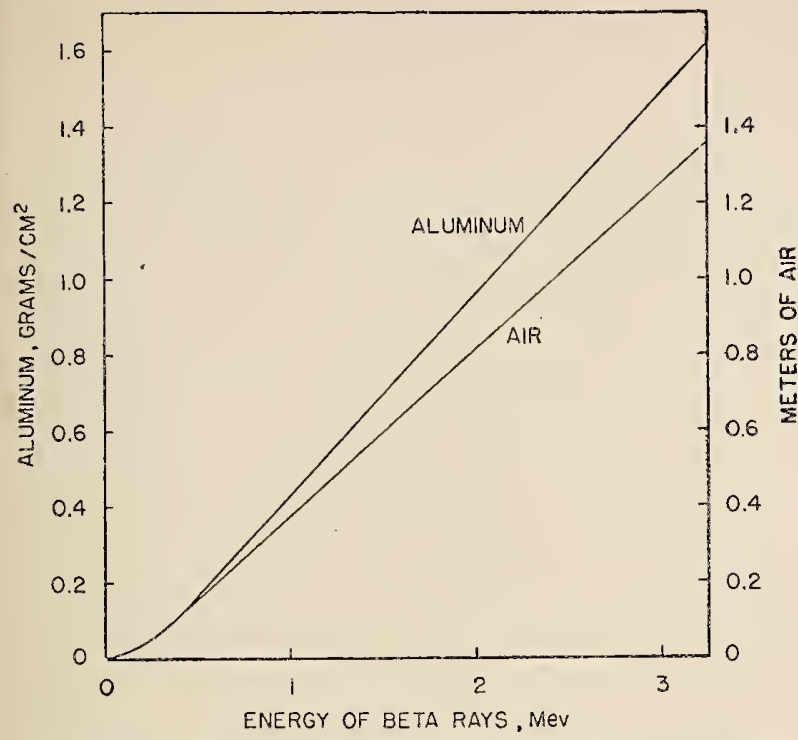

Figure 65. Graph showing the ranges in air and aluminum of beta rays of various energies.

The ranges of beta particles for energies greater than $0.5 \mathrm{Mev}$ are merely linearly proportional to the energy as, shown in figure 65 , where the range in aluminum and air are shown for energies up to $3 \mathrm{Mev}$.

\section{Absorption Method for Determining Energies of Beta Rays}

Feather [57] has shown that the maximum energy of a beta-ray spectrum can be determined approximately from the range in aluminum by use of the equation

$$
R=0.543 E-0.16 \text {, }
$$

where $R$ is expressed in milligrams per square centimeter and $E$ in microelectron volts, which gives the maximum energy of the beta rays as

$$
E_{\text {Max }}=\frac{R}{0.543}+0.294
$$

The range is determined by measuring the transmitted intensity through progressively increasing thicknesses of aluminum. If the intensity is plotted on semilog paper with the logarithmic scale along the $y$-axis and the corresponding thickness on the $x$-axis, an approximately straight line of negative slope is obtained. If, as is usual, gamma radiation is also emitted by the source, a point is found where the slope rapidly becomes much smaller, indicating that all beta rays have been absorbed, the residual effect arising from the gamma ra- diation. The thickness of absorber at which this change occurs is the value of $R$ to be used in eq 84. Figure 66 shows a typical absorption curve for beta rays in aluminum. By using the range of $0.4 \mathrm{~g} / \mathrm{cm}^{2}$, the energy comes out $1.02 \mathrm{Mev}$. The figure illustrates the uncertainty in this method, since it is difficult to select definitely the point at which beta rays are no longer de-

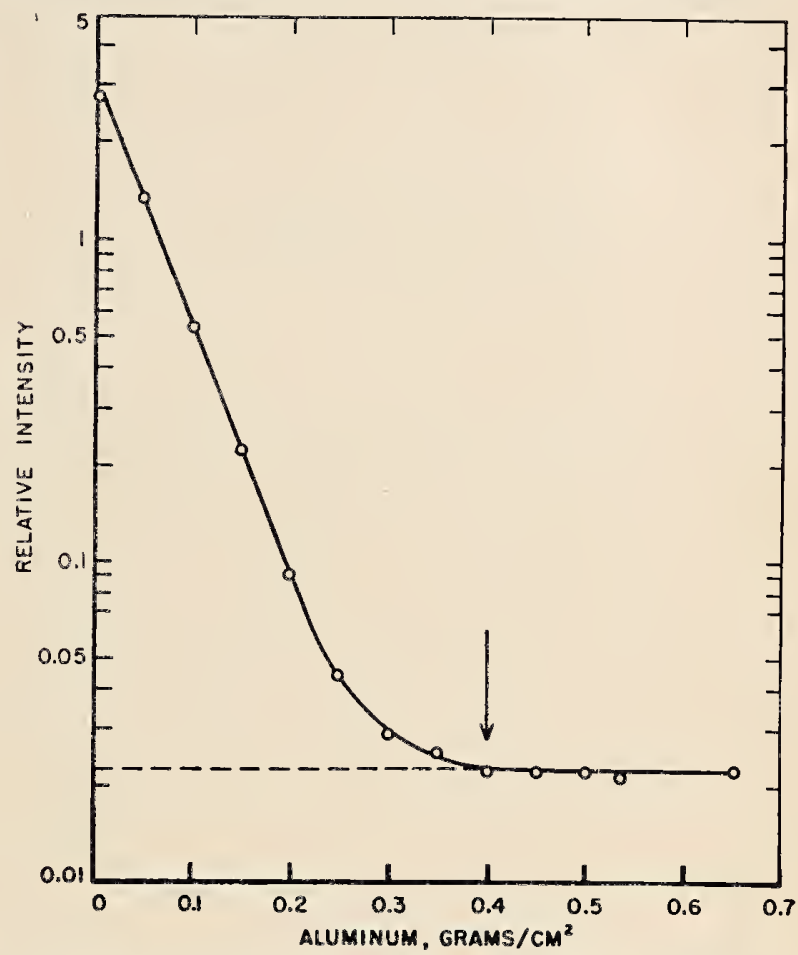

FIGURE 66. Graph showing the decrease in relative intensity of beta rays from $\mathrm{Zn}^{69}$ on passing through successive thicknesses of aluminum.

tected as the line representing the beta-ray absorption curves gradually merges into that representing the absorption of the gamma rays.

\section{Beta-Ray Energies by Magnetic Deflection}

For low-energy beta rays the maximum energies of the continuous spectra may be determined by the combination of a screen-wall Geiger-Müller counter and a solenoid arranged to produce a magnetic field, along the axis of the counter. This procedure has been described by Libby and Lee [58]. The arrangement is illustrated in figure 67. As the magnetic field is increased, the rate of counting decreases until finally, if gamma rays are also present, a minimum counting rate is observed where further increase in the magnetic field produces no additional effect. Plotting the counting rate for various currents in the solenoid yields curves similar to that shown in figure 68 , which 
shows results obtained from radioactive beryllium, indicating that $E_{\mathrm{Max}}=13 \mathrm{kev}$ for these particles. The value of the current for which the effect of the beta rays becomes zero gives the data for computing the maximum $H_{\rho}$ and the maximum energy of the beta particles. The

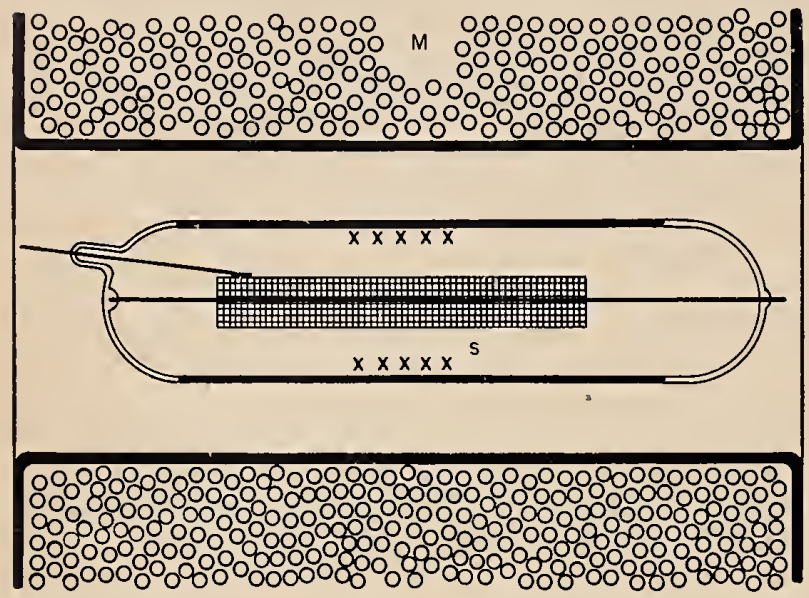

FiguRE 67. Diagram of apparatus for determining beta-ray ranges by magnetic deflection. $M=$ solenoid; $S=$ source mounted in Geiger-Müller counter.

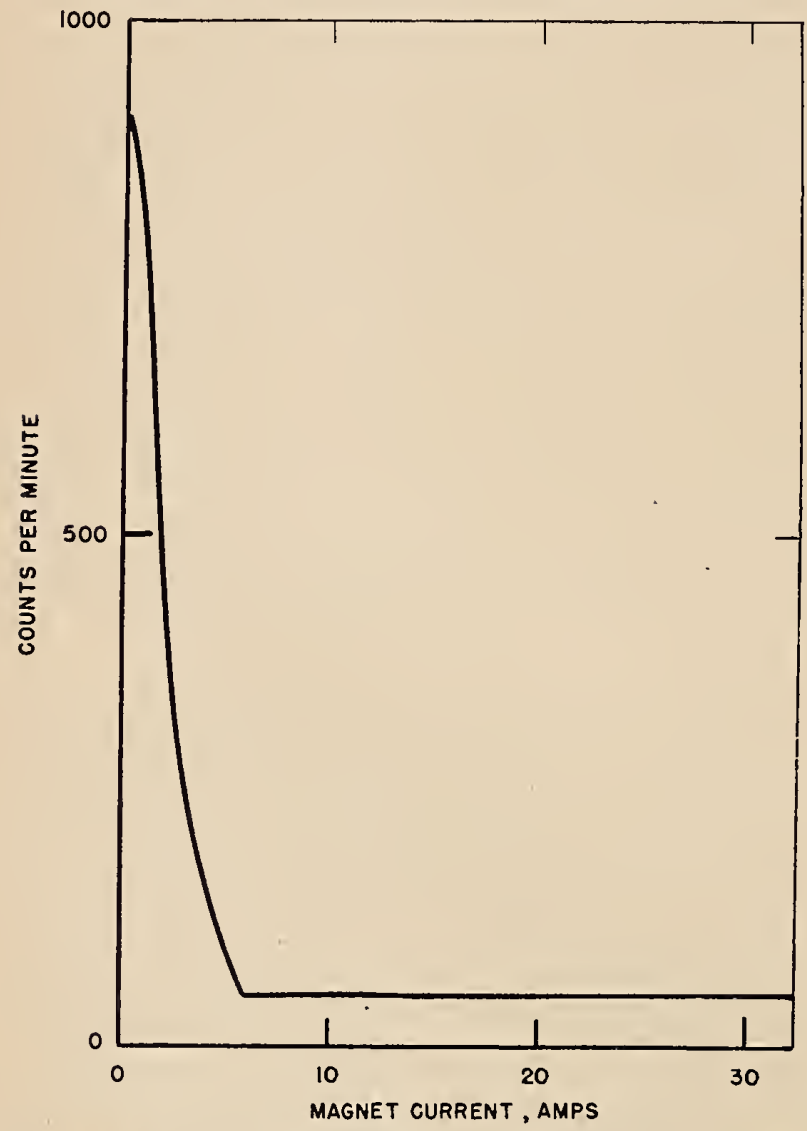

FIgURE 68. Curve obtained with arrangement shown in figure 67. method can be used for beta particles with maximum energies from 10 to $20 \mathrm{kev}$ up to $2 \mathrm{Mev}$.

\section{Positron}

This particle, discovered by C. D. Anderson [59] in 1932, has a mass and charge identical to that of the electron, with reversed sign. It is a common product of the disintegration of artificially radioactive isotopes. When a positron is emitted by the nucleus, the nuclear charge decreases by one unit, resulting in a decay product in a column one space to the left in the periodic table. The same result is produced by orbital electron capture by the nucleus, and these two modes of decay frequently occur in the same isotope resulting in the same product. Those radioisotopes that have a smaller mass than that of the neighboring stable isotopes show a pronounced tendency to decay by positron emission.

As is to be expected from the nature of the particle and its mode of production in radioactive decay, the positron spectrum of a radioisotope is very similar in form to the beta-ray spectrum of beta-ray emitters, i.e., it is distinguished by a continuous distribution with a definite characteristic maximum end point. These spectra can be studied in the same types of spectrometers used for studying beta-ray spectra. However, in the semicircular magnetic focussing spectrographs the magnetic field must, of course, be reversed.

\section{Annihilation Radiation and Pair Production}

Positrons exist in a free state only a fraction of a second when in the presence of electrons with which they may combine. In the interaction of the positron and electron both disappear. The two elementary masses are converted each into a gamma quantum in this process according to the relation $m_{0} c^{2}=h v=0.511$ Mev. This gamma radiation is known as annihilation radiation. The conservation of momentum requires that the two quanta be emitted in opposite directions with a net momentum of zero, since the particles at the instant of annihilation have zero momentum. Annihilation radiation is observed in connection with all isotopes disintegrating by the emission of positrons.

This annihilation of a positron and an electron to produce two quanta of gamma radiation is a reversible process. Whenever gamma radiation with energy in excess of $1 \mathrm{Mev}$ is incident on matter, there is a probability that some of the quanta will be absorbed to produce an electron pair, one electron and one positron. This probability increases with the energy of the 
gamma-ray quanta. When gamma rays with energies above $3 \mathrm{Mev}$ are absorbed approximately one-half the energy is given up to produce electron pairs.

\section{Mesotrons}

These particles, discovered by Anderson [60] in cosmic-ray studies with a cloud chamber, are now preferably called mesons. The designation of mesotron arose from the fact that their properties are closely related to those of the electron. In the original observations, Anderson noted particles that had a high specific ionization, revealed by dense tracks in the cloud chamber. However these particles were of such high energy that the magnetic field used for the observation produced only a slight curvature. By using cloud chambers controlled by Geiger-Müller counters, Street and Stevenson [61] were able to photograph these particles near the end of their range where measureable curvature in a strong magnetic field was obtained. They estimated the mass of these particles as about 200 times that of an electron, assuming that they carry the same charge as the electron. LePrince-Ringuet [62] and coworkers have measured this mass as $240 \pm 20$ times that of the electron.

It has been shown from cosmic-ray investigations of the absorption of mesotrons in the atmosphere and in lead that this particle is radioactive. In passing through matter, a fraction of the particles disappear by other processes than absorption and this fraction increases with the time required to pass through an absorber of given total density per unit area. In this respect air absorbs mesotrons more readily than lead. This has been interpreted as meaning that the mesotrons are unstable and undergo spontaneous disintegration. On this basis the mean life has been computed as of the order of $2 \times 10^{-6} \mathrm{sec}$. The mean free path in air is of the order of $10,000 \mathrm{~m}$.

There is evidence that both positively and negatively charged mesotrons exist, the relative numbers of each being approximately equal at sea level. Rasetti [63] has observed that there is about one disintegration electron for every two mesotrons absorbed. This can be interpreted theoretically on the basis that negative mesotrons have a high probability of nuclear absorption and that only the positive mesotrons disintegrate by ejecting an electron. Much of the information regarding the properties of the mesotron is still in the speculative stage.

These particles have also been called "variotrons" because of their variable mass and instability. Masses ranging 100 to 3,000 times those of the electrons have been reported from observations of cosmic-ray mesotrons. It has become customary to refer to the heavier primary mesons as " $\pi$-mesons" and the secondary particles of lower mass as " $\mu$-mesons." Recently " $\sigma$ mesons" of masses of the order of 7 to 20 electronic charges have been reported [64]. Some of the most recent measurements have used the photographic emulsion technique for determining the range and specific ionization of these particles, giving information regarding their initial energies and masses.

\section{Artificial Production of Mesotrons}

The rest energy of the mesotron can be computed from the estimated mass. Assuming that this mass is 240 times that of an electron, which has a rest energy of $0.511 \mathrm{Mev}$, the rest energy of the mesotron is about $120 \mathrm{Mev}$. Therefore, accelerators that can produce radiation or accelerate particles with this or greater energy can be expected to generate mesotrons. This expectation has been confirmed by use of the 184-in. cyclotron at Berkeley, in 1948 [65]. The observations were made with photographic plates placed near a target bombarded with alpha particles with an energy of $384 \mathrm{Mev}$. The tracks obtained revealed the same type of scattering and variation of grain density with residual range found in cosmic-ray meson tracks with the frequent appearance of characteristic stars at the end of the range. By using magnetic deflections, an estimate of the mass was determined as roughly 315 electron masses.

\section{Gamma Rays}

Gamma rays are emitted with sharply defined energy, which is analogous to the lines of optical spectra. Therefore the nucleus must be regarded as a quantized system that can exist in a number of states of energy with definite probabilities of transitions to states of lower energy, the difference in energy being emitted as electromagnetic radiation. This type of nuclear radiation is known as gamma radiation. These radiations are emitted in general when a nucleus undergoes a radioactive transformation into a new nucleus by the emission of beta rays, positrons, or alpha particles. The new nucleus usually is left in an excited state, that is with an excess of energy above the normal or ground state, and the excitation energy is radiated by one or more transitions within the nucleus until the ground state is reached.

Since the differences in energy between the various levels of the nucleus are much greater than those of the electron levels of the atom, responsible for optical and X-ray spectra, the energies of gamma rays are much higher and their wavelengths much shorter. Therefore, the usual methods of spectroscopy cannot be used to measure the wavelengths of gamma rays, 
except for those that happen to have relatively low energies.
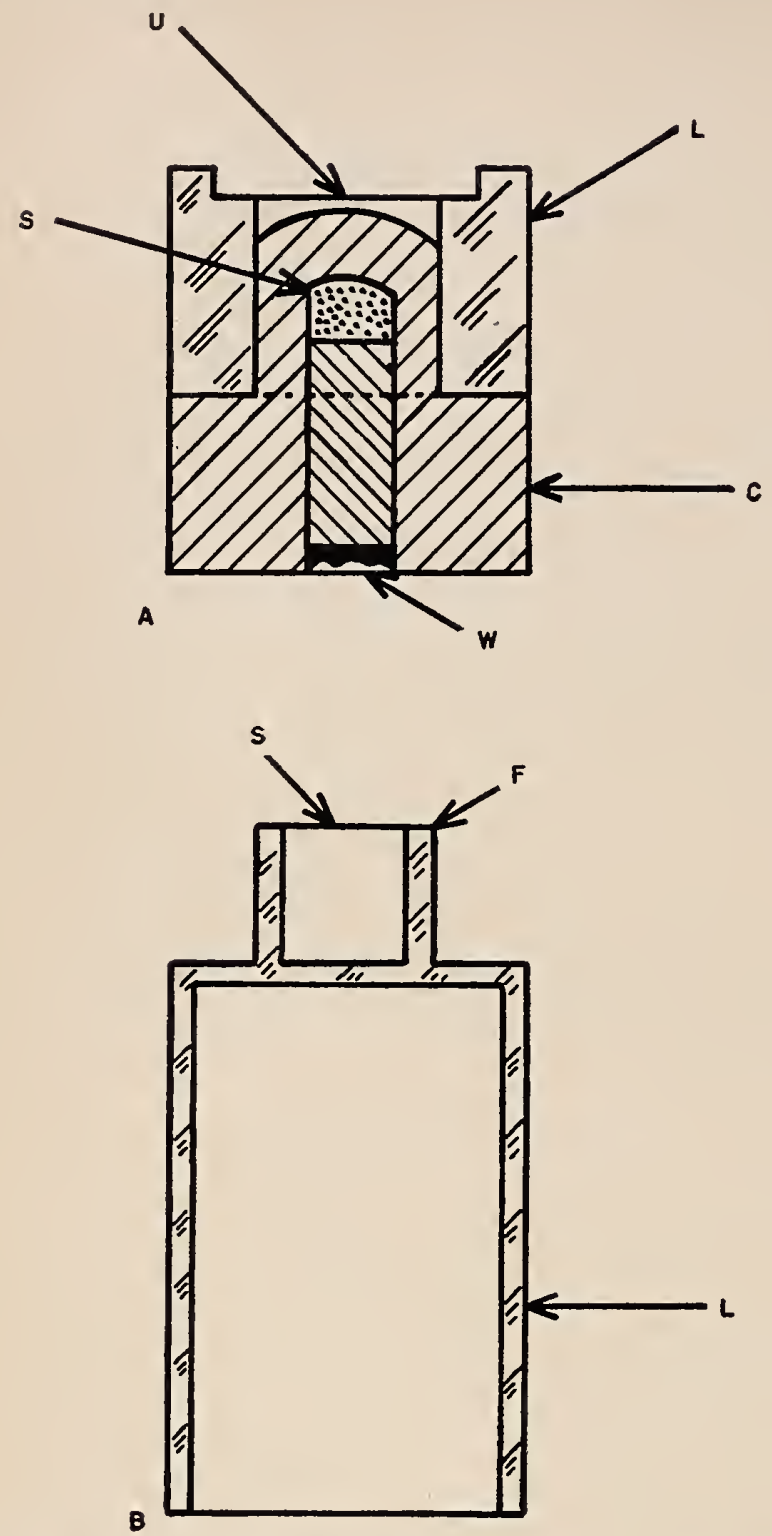

FIGURE 69. Cross section of source holder for beta-ray spectrometer ( $f g .59$ ).

A, Gamma-ray source. $U=$ Uranium radiator; $S=$ radioactive A, Gamma-ray source. $U=$ Uranium radiator; $S=$ radioactive W=wax. $\bar{B}$, Beta-ray source. $S=$ radioactive source; $F=$ foil; $\mathrm{L}=$ plastic mount for foil.

For the higher energies the phenomenon of internal conversion has been utilized to determine the energy of gamma rays. The procedure has been brought to a high degree of accuracy by the work of Ellis, using the semicircular beta-ray spectrograph to measure the energy of the conversion electrons. Since the conversion electrons have energies equal to the original energy of the gamma ray, minus the binding energy of the electron level in which conversion occurs, one gamma ray may produce several lines in the conversion spectrum.

Whereas internal conversion is fairly pronounced for all the heavier isotopes in the region where natural radioactive elements pre dominate, it becomes more rare among isotopes of lower atomic number. Therefore little or no internal conversion occurs for gamma rays from many of the artificially radioactive isotopes. The gamma-ray energies for isotopes that do not emit conversion electrons can be measured by permitting these gamma rays to undergo conversion in thin layers of material of high atomic number. The common practice is to use such elements as gold and uranium for radiators mounted with respect to the radioactive source, as shown in figure 69 . The brass capsule is thick enough to absorb all beta rays from the source so that all electrons emitted are generated either by photoelectric conversion in the radiator, quite similar to the internal conversion process, or by Compton recoil from the radiator. Both types of electrons are usually present. When the source holder shown in figure 69 is inserted in a magnetic lens electron spectrometer, a curve similar to that shown in figure 60 is obtained in which the conversion electrons appear as sharp peaks superimposed on the background of Compton electrons.

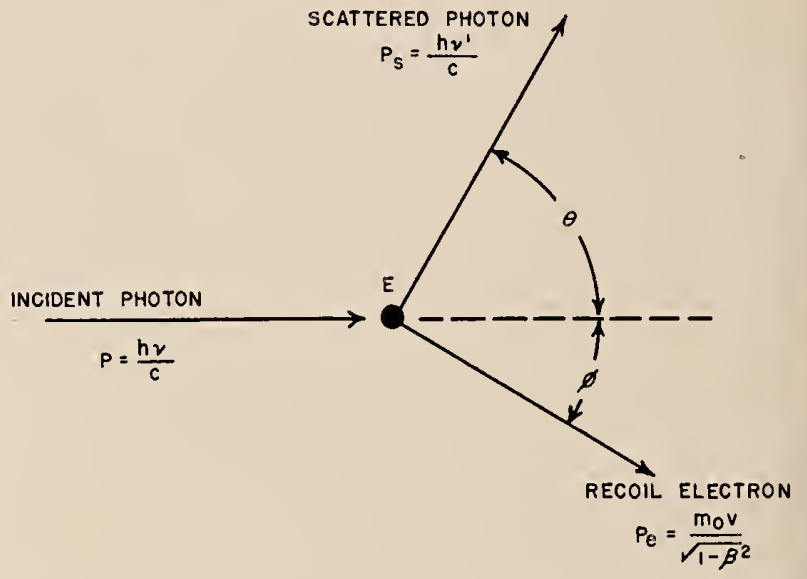

FIGURE 70. Diagram showing relation of scattered photon to recoil electron in Compton scattering. $\mathrm{E}=$ =electron.

These Compton recoil electrons are generated by virtue of the corpuscular nature of electromagnetic quanta. Compton proved that when electromagnetic radiation impinges on an electron, with part of its energy being scattered in a direction different from the original, the electron recoils at an angle with the direction of the original quantum of radiation, as shown in figure 70 . In explaining this phenomenon quantitatively, it is necessary to assume that mass and momentum be ascribed to the incident quantum. 
If $E=h v$ is the energy of the incident quantum, its mass is $h_{v} / c^{2}$, and its momentum is $h_{v} / c$. The scattered electron of rest mass $m_{0}$ has a mass of $m_{0} \sqrt{1-\beta^{2}}$, a momentum of $m_{0} v$ $\sqrt{1-\beta^{2}}$ and an energy of $m c^{2}-m_{0} c^{2}$, where $\beta=v / c$, the ratio of the velocity of the electron to that of light.

The conservation of energy requires that

$$
h_{1}=h_{\gamma^{\prime}}+m c^{2}-m_{0} c^{2},
$$

where $h_{r^{\prime}}$ is the energy of the scattered quantum. The conservation of momentum, which can be resolved into two components, one parallel and the other perpendicular to the direction of the incident quantum, requires that

$$
\frac{h_{v^{\prime}}}{c}=\frac{m_{0} v}{\sqrt{1-\beta^{2}}} \cos \phi+\frac{h_{v^{\prime}}}{c} \cos \theta,
$$

for the parallel component and

$$
\frac{m_{0} v}{\sqrt{1-\beta^{2}}} \sin \phi=\frac{h_{v^{\prime}}}{c} \sin \theta,
$$

for the perpendicular component of the momentum. From these relations the change of wavelength corresponding to the change from $v$ to $r^{\prime}$ was deduced as given in Angstrom units.

$$
\begin{aligned}
\lambda^{1}-\lambda & =0.0241 \quad(1-\cos \phi), \\
\delta \lambda & =0.0482 \sin ^{2} \phi /{ }^{2} .
\end{aligned}
$$

This quantity, for a given angle of scattering, is independent of the energy of the incident radiation.

There is a recoil electron at the angle $\phi$ for each quantum scattered at the angle $\theta$, and the recoil electrons produced by quanta scattered directly backward have the maximum energy. The value of $\delta \lambda$ for the direct backward scattering is $0.0482 \mathrm{~A}$.

From the relation $e V=h c / \lambda$ this energy comes out $0.257 \mathrm{Mev}$. Therefore, if $E$ represents the energy of the incident and $E^{\prime}$ the energy of the scattered quantum in million electron volts

$$
\frac{1}{E^{\prime}}-\frac{1}{E}=\frac{1}{0.257}=3.9,
$$

or

$$
E^{\prime}+3.9 E E^{\prime}=E,
$$

from which we obtain

$$
E^{\prime}=\frac{E}{1+3.9 E}
$$

Therefore the Compton recoil electrons may be used to determine the energy of a gamma ray. The distribution of energies of the recoil electrons, as observed in a magnetic lens electron spectrometer, has a well-defined maximum for a single gamma ray. This energy is $E-E^{\prime}$ and can be determined from the maximum $H_{\rho}$ of the observed distribution. Representing the energy by $w$ we have

$$
E-E^{\prime}=w
$$

or

$$
E-\frac{E}{1+3.9 E}=w
$$

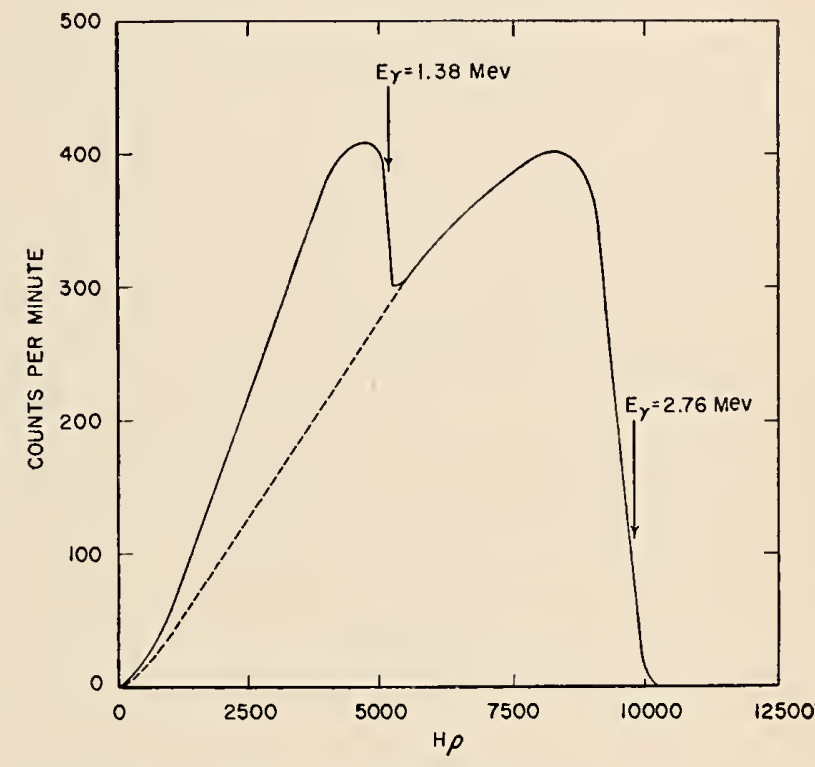

FigURE 71. Graph showing distribution of Compton electrons from two gamma rays as measured in an electron spectrometer.

which can be solved for $E$, the energy of the incident gamma ray. A typical distribution of recoil electrons obtained by Siegbahn [67] in a magnetic lens spectrometer is shown in figure 71. The two gamma rays are from $\mathrm{Na}^{24}$, and the recoil electrons are from a copper radiator.

\section{Disintegration Schemes}

A fairly complete picture of the transitions in nuclear energy resulting from a radioactive transformation can be obtained from a knowledge of (1) the number of gamma rays emitted, their energies, and the order in which they are emitted (or knowledge of which gamma rays are emitted simultaneously); (2) the maximum energy, or energies, of the beta rays emitted; and (3) which beta rays are associated 
with the various gamma rays. This representation is known as a disintegration scheme.

A typical nuclear disintegration scheme, that for $\mathrm{Sc}^{46}$, is shown in figure 72. In this representation the energy lost by the removal of the mass of the beta ray $(0.511 \mathrm{Mev})$ from the $\mathrm{Sc}^{46}$ nucleus is not shown, for simplicity. The various factors that enter into this scheme are determined as follows: The maximum energy of the beta rays and the fact that only one group of beta rays is emitted is ascertained by measuring the beta-ray energies in a magnetic lens spectrometer. Figure 72 shows the results of such a measurement. The energies of the two gamma rays are determined by measuring in the same instrument the energies of the conversion electrons. This spectrum from a uranium radiator is shown in figure 60 . It

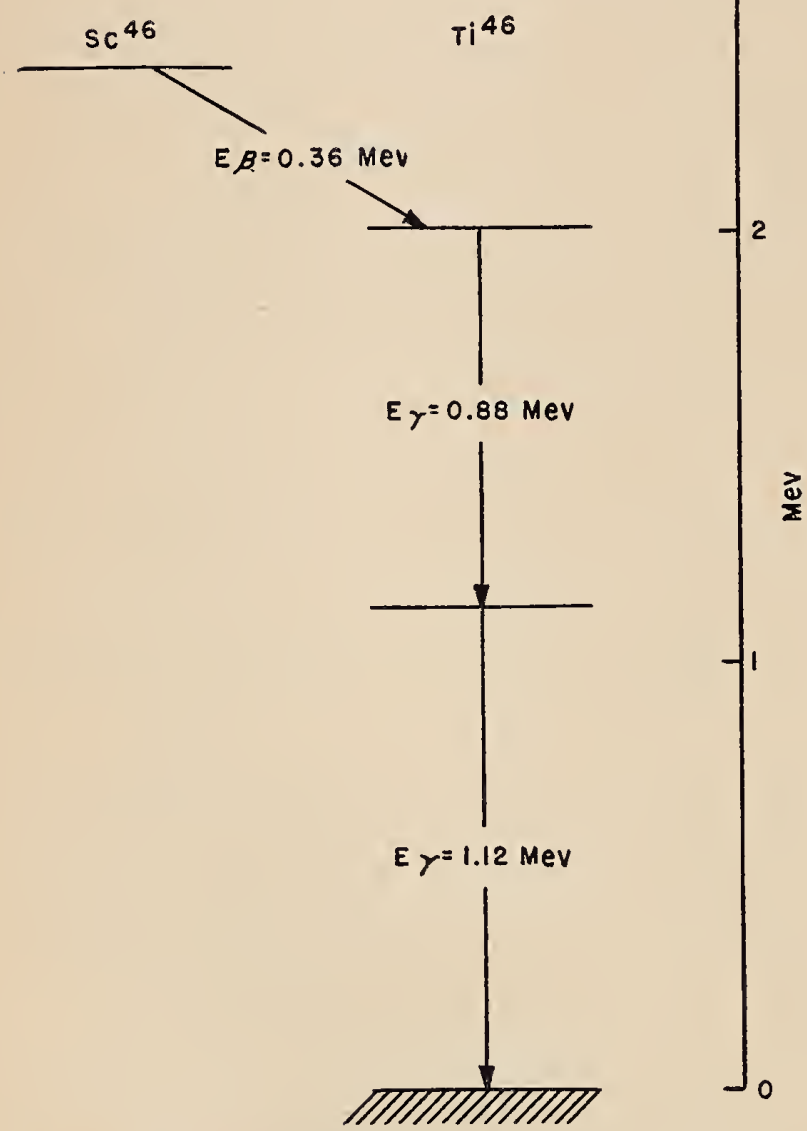

Figure 72. Disintegration scheme for $S c^{46}$.

can also be established that the relative intensity of each gamma ray is approximately the same from a study of the conversion electrons. To justify the scheme as shown in figure 72 it is only necessary, in addition, to show that the two gamma rays are emitted simultaneously and at the same time as the corresponding beta ray. These facts can be established by coincidence measurements.

\section{Concidence Measurements}

A method for determining the simultaneous emission of different types of radiation from the atomic nucleus was first described by Bothe and von Baeyer [68]. By coincidence measurements, it is possible to determine which gamma rays are emitted simultaneously with a given beta ray, where two or more modes of beta disintegration occur; or to show for a simple betaray disintegration whether the gamma rays accompanying them are emitted "in cascade," in which case systematic coincidences between the gamma rays are to be expected.

Coincidences between the various types of radiation are determined by use of an electronic coincidence arrangement, usually based on the Rossi [69] circuit devised for determining cosmic ray coincidences. A diagram of this circuit for 2-fold coincidences is shown in figure 73. It is important that the resolving time, $\tau$, of the coincidence circuit be made short, where $\tau$ is defined by the relation

$$
A=2 N_{1} N_{2}, \tau,
$$

in which $A$ is the accidental coincidence rate between counters 1 and 2 when the individual counting rates are $N_{1}$ and $N_{2}$. However, if $\tau$ is made much less than $10^{-6}$, second true coincidences are lost as a result of the fluctuations in the time required for the initiation of a pulse after the radiation has traversed the counter. This fluctuation may approach $10^{-6} \mathrm{sec}$ in some counters."

Short resolving times in coincidence circuits are most conveniently obtained by what is called electrical differentiation of the counter pulse. This procedure is described by Lewis [70]. If a slow pulse, say of $10^{-4}$ sec duration, is passed through a resistance capacity filter of the form shown in figure 74 , in which the time constant $C R$ is made short compared with the desired resolving time, it will be converted into a sharper pulse, to which is attached a long tail. The advantage of electrical differentiation is shown in figure 75. At $A$ two pulses are shown, one solid and one dotted, which have a durition of about $10^{-4} \mathrm{sec}$, but which are displaced along the time axis by about $4 \mu \mathrm{sec}$. Here the two voltage peaks are not resolved, since the dotted pulse rises to approximately the maximum voltage before the pulse represented by the solid curve has fallen noticeably. If these two successive pulses are passed through the electrical filter shown in figure 74 , the pulses will then have the forms shown at $B$, where a 


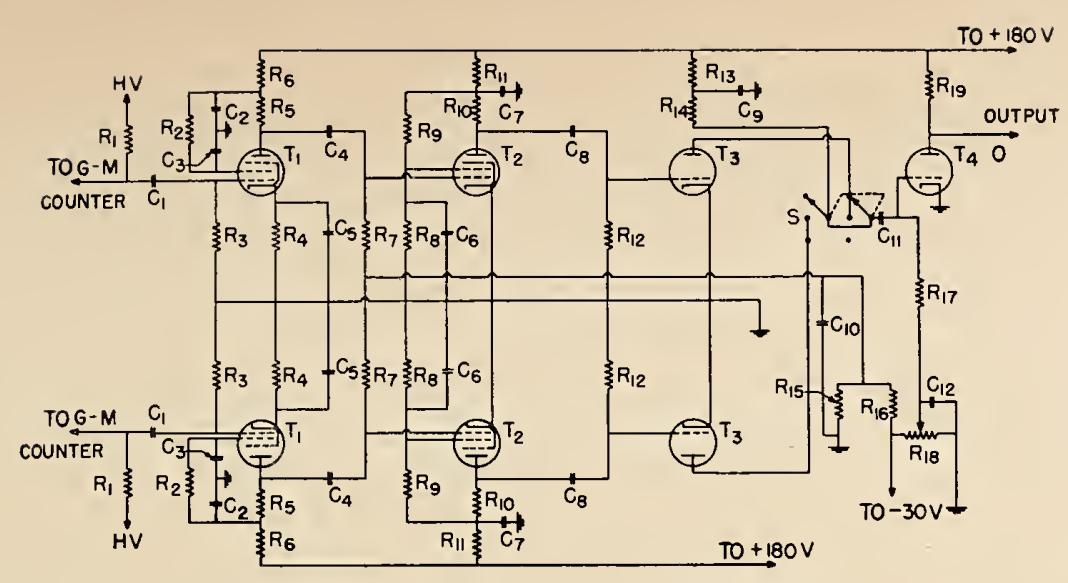

FiguRe 73. Wiring diagram of a two-fold coincidence circuit.

$R_{1}, R_{3},=0.5$ megohm; $R_{2}=0.1$ megohm; $R_{4}=220$ ohms; $R_{5}, R_{7}, R_{10}=25,000$ ohms;

$R_{6}, R_{11}, R_{13}=5,000$ ohms; $R_{15}=15,000$ ohms; $R_{8 ;}, R_{9}, R_{12}, R_{14}, R_{16}, R_{17}$

$R_{15}, R_{19}=60,000$ ohms; $C_{1}=50 \mathrm{mmfd} ; C_{2}, C_{7}, C_{9}=2 \mathrm{mfd} ; C_{3}, C_{6}, C_{10}$,

slight separation of the two peaks is evident. Passing the pulses shown at $B$ through a second similar filter yields the pulse forms shown at (!, where the two pulses are clearly separated. The variation in pulse heights can be levelled by appropriate electronic circuits, and the effect of the low amplitude after-pulse shown at about $80 \mu$ sec can be eliminated by suitable bias. When pulses that have a duration of the order of a microsecond result from the differentiation of pulses that are of the order of $10^{-4}$ sec, the amplifier must have a reasonably constant amplification over a wide band of frequencies extending from less than $10^{4}$ to well above $10^{6}$ cycles/sec. Great care is required in the selection and location of components in amplifiers of this type. However, for coincidence counting strict linearity is not required. Actually it is desirable that all pulses have the same height when arriving at the mixing stage of the Rossi coincidence circuit, regardless of their size at intermediate points.

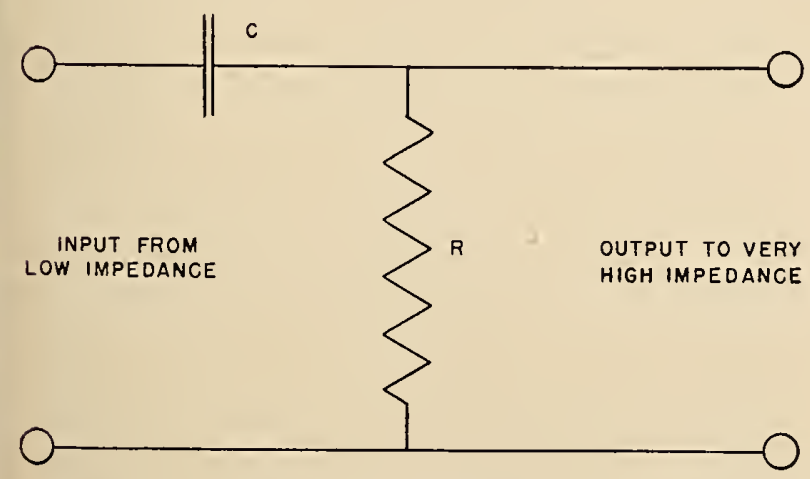

Figure 74. Electrical filter circuit for electrical differentiation of a pulse.

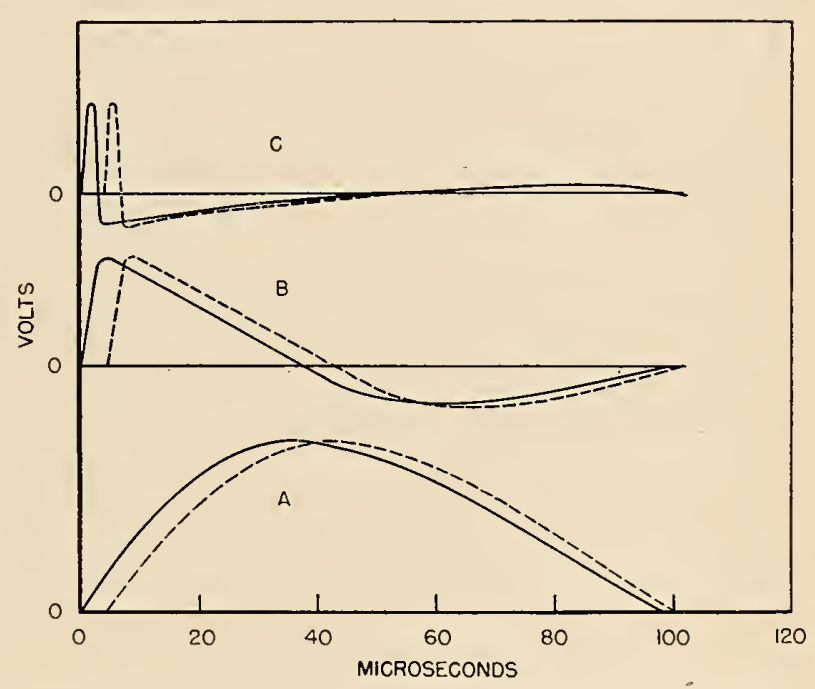

FIGURE 75. Diagram showing the results of suceessive differentiations of a slow pulse.

\section{Absolute Calibration of Gamma-Ray Sources by Coincidences}

When the disintegration scheme is known, radioactive isotopes with simple modes of disintegration can be calibrated absolutely by coincidence measurements. The procedure is as follows. Let $N_{k}$ be the number of nuclear gamma rays emitted per unit time, which for a simple disintegration scheme is also the number of nuclear disintegrations per unit time and is equal to $N_{e}$, the number of beta particles emitted per unit time. A given gamma-ray counter will yield a counting rate of

$$
Y=P_{k} N_{k} \text {, }
$$


where $P_{k}$ is the efficiency, or probability, of response to gamma rays of the energy corresponding to those under measurement. Similarly, a beta-ray counter will give a counting rate

$$
Z=P_{e} N_{e}=P_{e} N_{\vec{k}}
$$

where $P_{r}$ is the efficiency of the beta-ray counter.

In a coincidence arrangement the number of beta-gamma coincidences, $K$, will be

$$
K=P_{k} P_{e} N_{k} \text {. }
$$

But $P_{k}=Y / N_{k}$, and $P_{e}=Z / N_{k}$.

Therefore, we obtain

$$
N_{k}=\frac{Y Z}{K},
$$

which states that the disintegration rate is equal to the product of the counting rates of the two counters divided by the coincidence rate.

As an example, for a simple scheme consisting of a beta ray followed by a single gamma ray, let us suppose that in a coincidence arrangement the counts in the beta-ray counter are

$$
Z=2030 / \mathrm{min}
$$

and the gamma-ray counts in the gamma-ray counter are

$$
Y=109 / \mathrm{min},
$$

and the coincidences $K$ are $1.99 / \mathrm{min}$.

Then

$$
N_{k}=\frac{2030 \times 109}{1.99}=1.11 \times 10^{5}
$$

disintegrations per minute.

\section{Measurement of Gamma Radiation}

Prior to the advent of artificial radioactivity and accelerators for the production of nuclear radiation, radium was the principal source of gamma radiation. Therefore, the custom developed of referring to gamma radiation in terms of milligrams of radium or the equivalent millicuries of radon. Whenever this practice referred to the source, such a procedure was legitimate, in other words, an unknown amount of radium can be calibrated in terms of the gamma rays from a definite weight of radium. However, this manner of specification has been distorted to refer to the radiation, although it is obvious that such factors as distance from source, filtration, and type of detecting instrument are all involved in specifying the radiation from a given amount of radium. Furthermore, no absolute comparison of the gamma radiation from radium and from mesothorium, which emits gamma rays of quite different energies, was possible under the above procedure. This problem has become more complex with the development of artificial radioactivity.

Much of this difficulty has been eliminated by use of the roentgen, originally introduced to measure X-radiation in terms of its ionizing effect, primarily as a unit of tissue dosage. It is defined as a quantity of X- or gamma-radiation such that the associated corpuscular emission per $0.001293 \mathrm{~g}$ of air produces in air ions carrying 1 electrostatic of electricity of either sign. Thus, we see that the roentgen gives a unit to measure the effect of gamma rays on a selected detector, namely, air, under standard conditions. Therefore, it is not correct to state that 1 roentgen/sec is a measure of the intensity of the gamma radiation. It is actually that intensity of gamma radiation, of any energy that will produce 2.09 ion pairs $/ \mathrm{cm}^{3} / \mathrm{sec}$. The actual energy flux in the beam is related to the roentgens per second through the variation of ionization with energy of the radiation. Furthermore, the ionization effect as expressed in roentgens is not linearly proportional to other effects that may be produced by the radiation as, for example, the blackening of photographic emulsion, which varies markedly with the energy of the gamma rays over a considerable range.

The roentgen unit can be used, if a definite distance from the source and a unit of time are specified, to measure the strength of any source of gamma rays in an unambiguous manner. For this purpose, 1 roentgen $/ \mathrm{hr}$ at $1 \mathrm{~m}$ ( $\mathrm{rhm}$ ) has been suggested by the National Bureau of Standards as a suitable unit. Under equal conditions of filtration, amounts of any given isotope emitting gamma rays can be compared. In fact, it is about the only practicable way to measure the quantity of an isotope emitting gamma rays for which the disintegration scheme is unknown.

Although the roentgen is clearly defined it is not easy to measure it in terms of its definition, which assumes that the total ionization of all corpuscular radiation is measured, which would require, in some cases, ionization chambers the size of a large room. However, small chambers can be used if they do not disturb the distribution of secondary electrons existing before their introduction into the larger volume of air undergoing ionization by the radiation. This is the principle of the air-wall chamber. Furthermore, any specific design of chamber can be calibrated by comparison with an airwall chamber for radiations of various energies. 
Those ionization devices that conform to requirements for measuring radiation in roentgens can be calibrated by means of a standard preparation of radium. One milligram of radium covered by $0.5 \mathrm{~mm}$ of platinum gives 8.5 roentgens $/ \mathrm{hr}$ at $1 \mathrm{~cm}$. Other types of devices, such as Geiger-Müller counters, cannot be used to measure roentgens or roentgens per hour and cannot be calibrated reliably in such units for use with radiations of various energies, although for radiations with energy of the order of $1 \mathrm{Mev}$ and higher, an approximate calibration can be made.

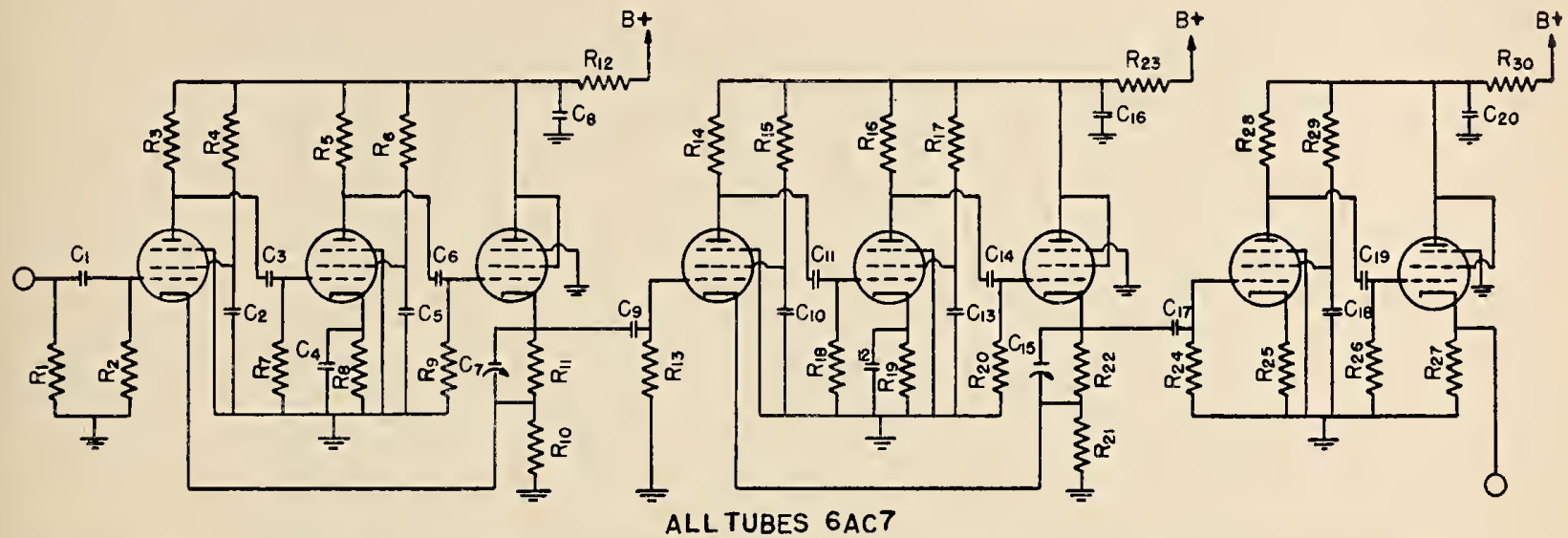

FIGURE 76. Wiring diagram of amplifier of short resolving time for counting pulses at high rate.

$R_{1}=75$ ohms; $R_{2}, R_{4}, R_{6}, R_{7}, R_{9}, R_{13}, R_{15}, R_{17}, R_{18}, R_{20}, R_{24}, R_{28}=680,000$ ohms; $R_{3}, R_{5}, R_{14}, R_{16}=4,300$ ohms; $R_{8}, R_{19}=150$ ohms; $R_{11}, R_{22}=5,000$ ohms; $R_{10}, R_{21}=100$ ohms; $R_{12}, R_{23}-2,200$ ohms; $R_{25}=56$ ohms; $R_{28}=3,000$ ohms; $R_{29}=30,000$ ohms; $R_{27}=1,000$ ohms; $R_{30}=510$ ohms; $R_{31}, R_{32}=1$ megohm; $R_{33}=1$ to 0.1 megohm; $R_{34}=2,000$ ohms; $C_{1}, C_{3}, C_{8}, C_{11}, C_{14}=0.0025 \mathrm{mfd} ; C_{2}, C_{5}, C_{8}, C_{10}, C_{13}$, $C_{18}, C_{18}, C_{20}=10 \mathrm{mfd} ; C_{4}, C_{12}=25 \mathrm{mfd} ; C_{7}, C_{15}=0$ to $10 \mathrm{mfd} ; C_{9}, C_{17}=0.00025 \mathrm{mfd} ; C_{19}=0.009 \mathrm{mfd} ; C_{219}, C_{22}=0.01 \mathrm{mfd} ; C_{23}=0.01$ mfd; all tubes $6 \mathrm{AC} 7$.

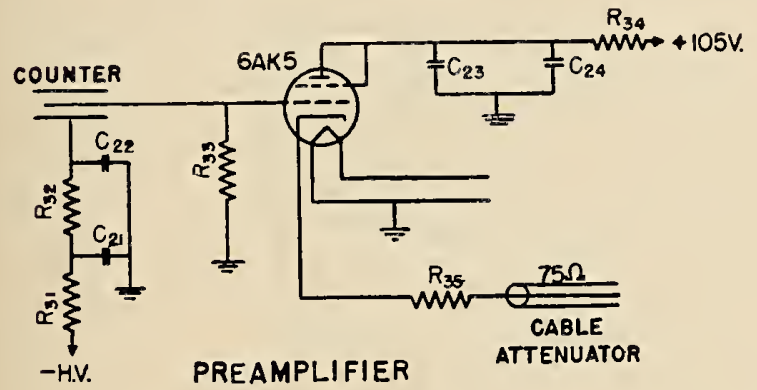

51. Counting Gamma Rays

Customarily Geiger-Müller type counters are used for counting gamma-ray photons by means of the secondary electrons produced mainly in the counter walls. This is the common procedure in coincidence counting for absolute calibration, and for studying disintegration schemes.

It has been pointed out by Benson [71] that some advantage is gained in using specially designed proportional counters for this purpose. The proportional counter has a shorter resolving time, which permits relatively high counting rates without excessive statistical losses. Proportional counters require carefully stabilized high-voltage supplies for accurate measurements. At fast counting rates a wideband high-frequency amplifier is required of sufficient gain to amplify the relatively small pulses from the proportional counter. Figure 76 is a diagram of the amplifier used by Benson, which has a power gain of about $80 \mathrm{db}$ over a band width of 4 megacycles/sec. The rate of rise of the pulses in the counter and amplifier is such that the pulse reaches two-thirds of maximum in $1 / 10 \mu \mathrm{sec}$.

Although various types of proportional counters with a great variety of gases at almost any pressure can be used with this amplifier, Benson has designed a counter that is useful for beta particles and protons as well as gamma rays. This counter is shown in figure 77.

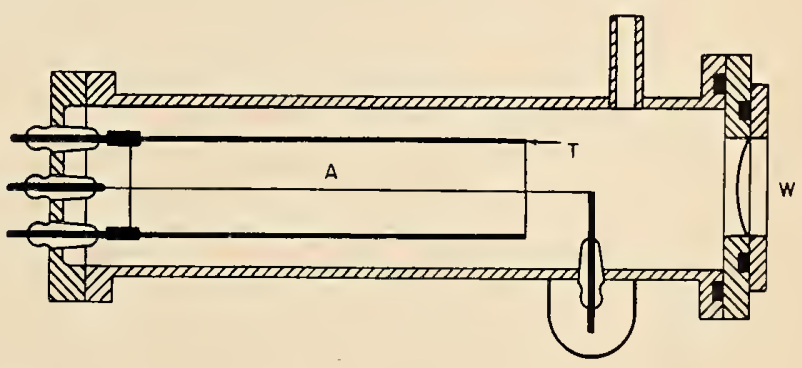

Figure 77. Cross section of a proportional counter for rapid counting of either particles or gamma rays. $A=$ Center wire; $T=$ cathode; $W=$ window.

\section{Fission Particles}

Since the nuclear fragments resulting from fission are atomic nuclei with large amounts 
of kinetic energy, they may be classed as a radioactive radiation, and many of their properties can be investigated by the same devices used to study alpha rays, for example.

Although nuclear fission was actually observed by Fermi [72] in 1934, he interpreted the radioactive products that he observed, when sess relatively high kinetic energies and therefore produced a very high specific ionization in gases. However, as is to be expected from particles of large mass compared with alpha particles, their range in air is only 1 or $2 \mathrm{~cm}$. An estimate of their energy is readily obtained by comparing their ionization per particle with

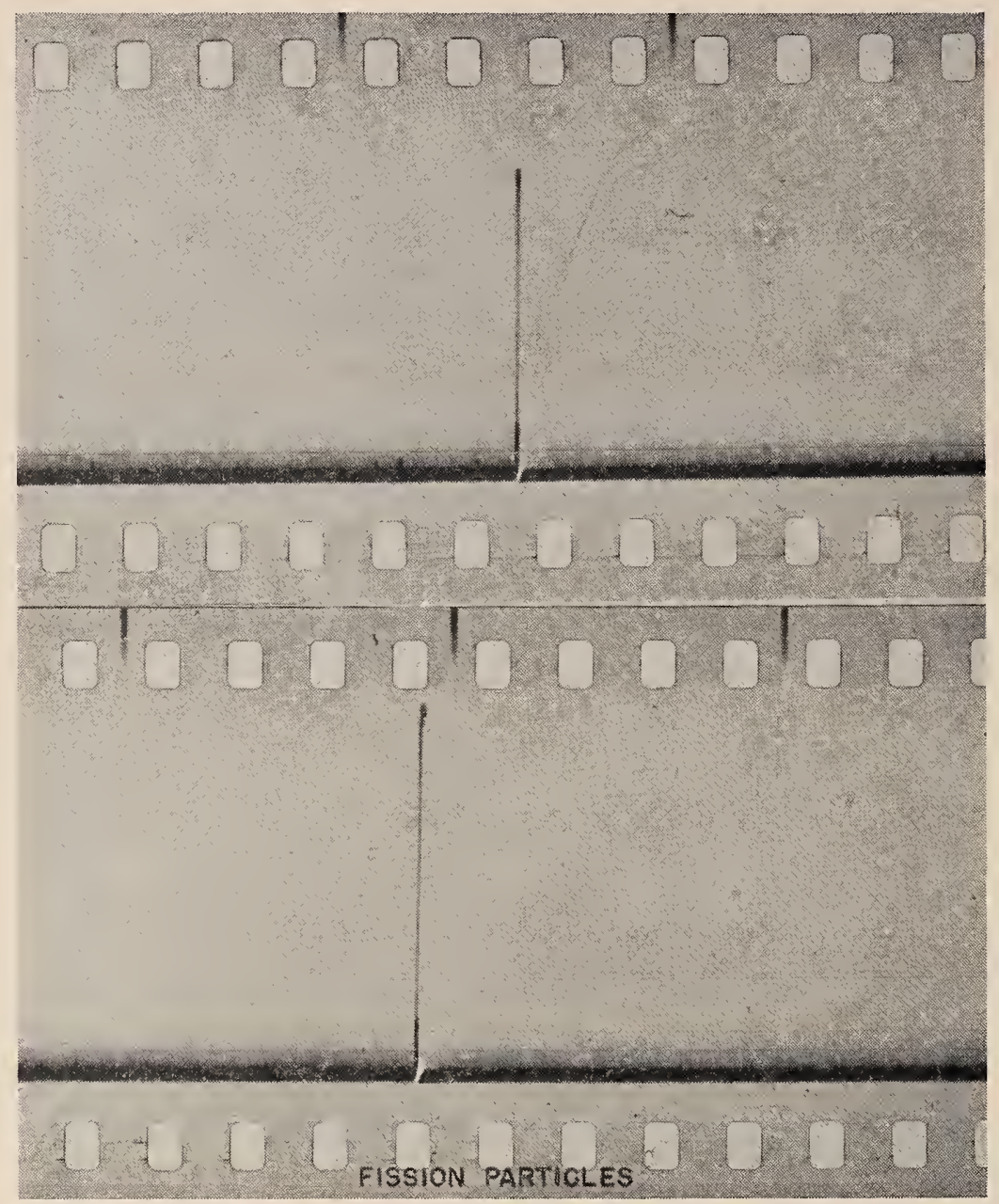

FiguRE 78. Oscillograph of a pulse produced by a fission particle.

uranium was exposed to slow neutrons, as transuranic elements resulting from simple neutron capture.

About 3 years later, Hahn, Meitner, and Strassman [73] by chemical studies, proved that one of these products was barium. Other investigators rapidly detected a number of elements near the center of the periodic table. However, it was not until 1939 that Hahn and Strassman [74] proposed the explanation of these results as the splitting of the uranium nucleus into two radioactive isotopes, each having roughly one-half the nuclear charge and mass of uranium.

These radioactive nuclei were found to pos- that of alpha particles. By using a linear amplifier and ion chamber, as shown in figure 7, an oscillograph record such as shown in figure 78 is obtained. The alpha particles that have an energy of approximately $4 \mathrm{Mev}$ barely show above the background, whereas the pulses due to fission particles extend nearly to the top of the record, indicating that these particles have approximately 20 to 30 times the energy of the alpha particle. Since two particles are released per fission, the total energy approaches $200 \mathrm{Mev}$.

Of the natural elements, available in quantities, only uranium and thorium undergo fission. On the other hand, there is considerable evi- 
dence to support the belief that all the heavier nuclei will undergo fission under proper conditions of neutron bombardment. The varied requirements for production of fission is illus-

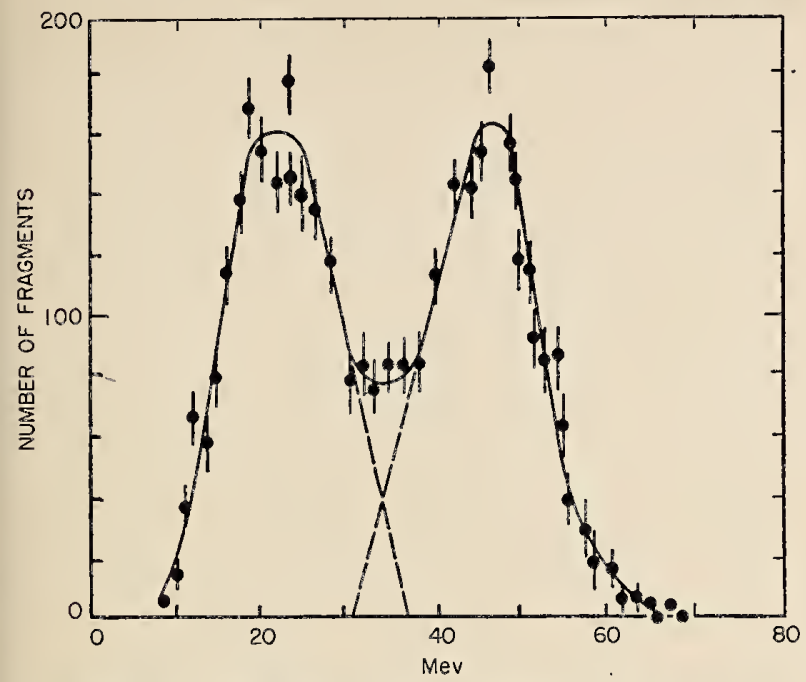

FIGURE 79. Curve showing the number of fission particles of various energies.

trated by the fact that $\mathrm{U}^{235}$ reacts readily to slow neutrons, and $\mathrm{U}^{238}$ requires neutrons with energy in excess of $1.5 \mathrm{Mev}$ for fission.

By deflecting fission fragments in the magnetic field of a cyclotron, Lassen [75] has determined the energy distribution of fission fragments. They fall into two well-defined groups of energies, which correspond to two

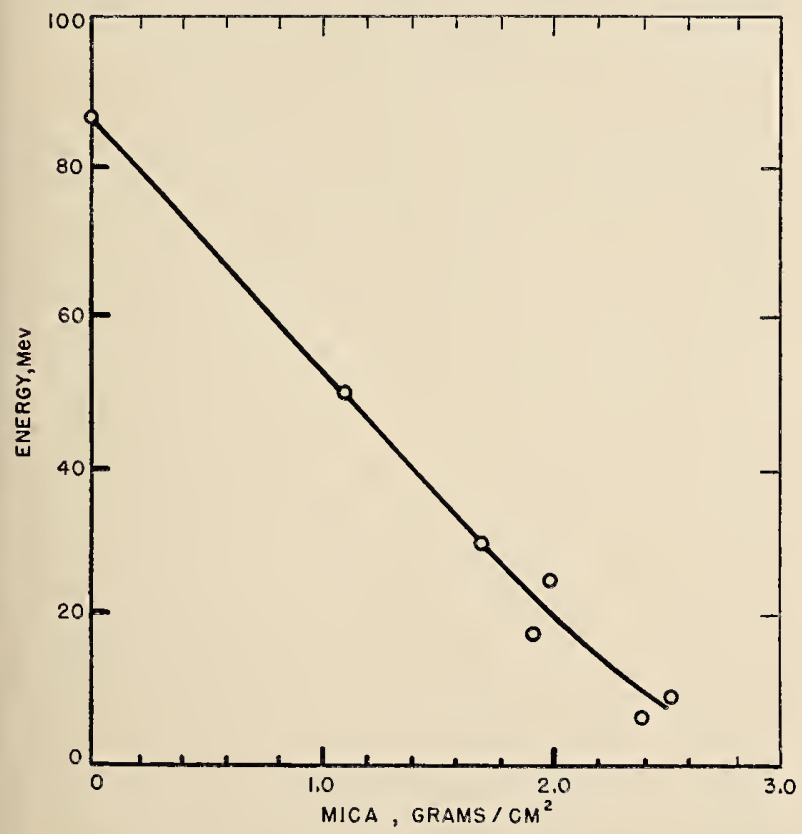

FIGURE 80. Energy-range curve for fission fragments of lighter mass. groups of masses. The curve in figure 79 shows the distribution after penetrating $0.79 \mathrm{mg} / \mathrm{cm}^{2}$ of mica, and therefore does not represent the distribution of the initial energies of the particles.

By using the same general method Lassen [76] obtains the equivalent of an energy-range curve for the light group of fragments. This is shown in figure 80 and illustrates the relatively low penetrating power of fission particles.

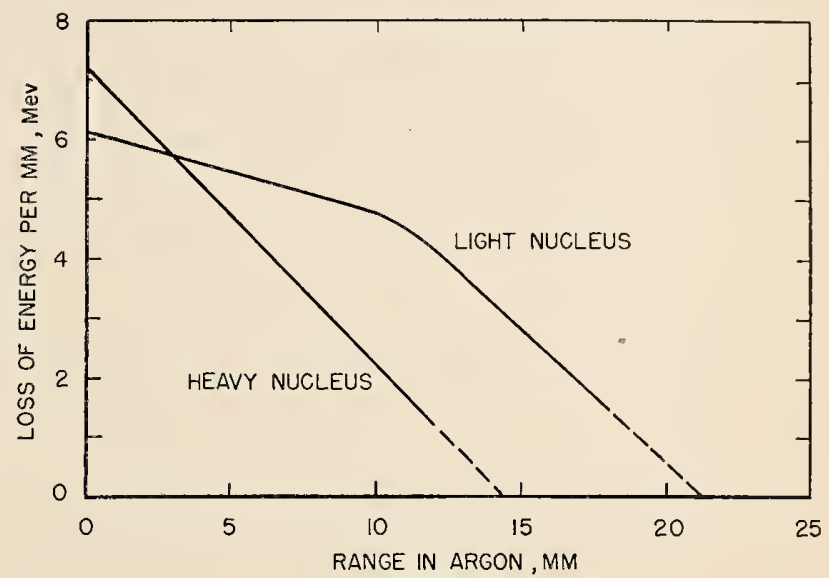

FIgURE 81. Curve showing the variation of specific ionization of fission particles with range in argon.

An investigation of the variation in specific ionization with range in argon by the same author [77] yields the curve shown in figure 81 .

The chamber used to obtain this data is shown in figure 82 . By use of the collimator only those particles moving in a direction almost perpendicular to the plates entered the chamber. The

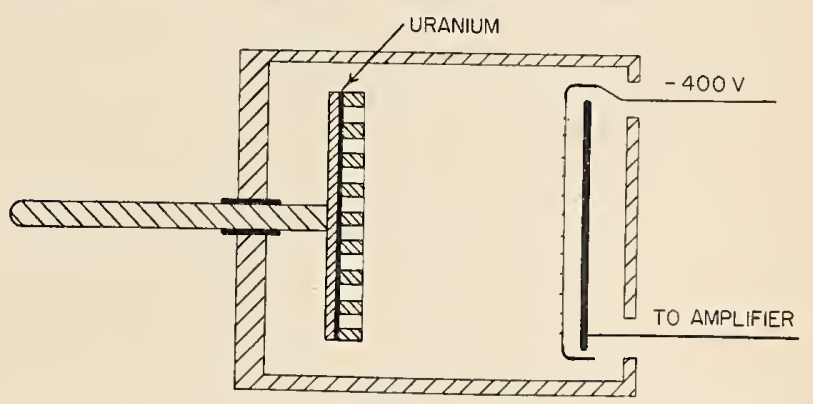

Figure 82. Diagram of chamber used to obtain data shown in figure 81 .

uranium and collimator could be moved measured distances along the axis of the chamber to determine the variation of specific ionization with the range of the particles.

\section{Measurement of Half-Value Periods}

The obvious method of determining the change of activity of a given sample at succes- 
sive intervals can be applied conveniently only for half-value periods in the range of from a few minutes to several days. For periods much smaller or larger than those in this range special methods and precautions must be used. The shorter periods cause difficulties in establishing an accurate time scale for the measurement and including a sufficient number of individual disintegrations in each separate observation to provide a reasonable statistical accuracy. The longer periods require that the measuring instrument maintain an accurate calibration over relatively long periods of time. For very long periods the specific activity is so low that there may be difficulty in arranging for sufficient intensity of the radiation to reach the detector. This may be particularly true for isotopes emitting only alpha, or beta radiation, or which emit soft gamma rays.

\section{Counter Measurement of Short Half- Value Periods}

For half-value periods in the range $10^{-4}$ to 1 sec Ward [78] has described a coincidence circuit by using a single counter. The principle involves the use of a coincidence type of circuit with a variable resolving time. Used with a single counter the circuit records as a "coincidence", whenever two pulses arrive within $T$ seconds of each other, where $T$ is the variable resolving time. In a circuit of this type the number of coincidences, $C$, is given for a random arrival of pulses by the relation

$$
C=n\left(1-e^{-n T}\right),
$$

when $n$ is the average number of pulses per unit time. If, for example, a small quantity of actinon is introduced into the counter the pulses will no longer occur at random, because each pulse produced by the disintegration of an atom of actinon will be followed by pulses from $A c A$ and succeeding members of this radioactive series. Assuming that all disintegrations occurring in the counter are recorded and the average time interval between disintegrations is large compared with the half-value period of $A c A$, which has the shortest life of the series, we see that each pulse due to the disintegration of an actinon atom will be followed by a pulse due to $A c A$, and the true separation of this pair of pulses will be much less than the average time interval between recorded pulses. For these conditions, the coincidence rate, $C$, is

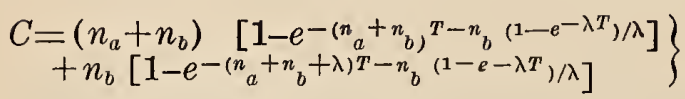

where $n_{a}$ is the number of random pulses per unit time and $n_{b}$ the number of pairs of corre- lated pulses per unit time, the correlation being expressed by the probability $P_{T}=\left(1-e^{-\lambda T}\right)$ that the second pulse of a pair follows the first within a time, T, and $\lambda$ is the decay constant to be measured.

The total counting rate is $n_{a}+2 n_{b}$. The significance of the rates $n_{a}$ and $n_{b}$ must now be considered. The time correlation of pulses produced by $A c B$ and succeeding members of the series has been ignored. Their half-lives are so much longer than the resolving time, $T$, of interest in determining the period of AcA, that these pulses are regarded as random counts included with the background rate of the counter in the value of $n_{\mathrm{a}}$. Some of the correlated pulses of An and AcA also contribute to $n_{a}$, since the geometrical efficiency of the counter is not 100 percent and because of the deadtime of the counter. The deadtime is of the order of $10^{-4} \mathrm{sec}$, which sets a limit for the shortest period that can be measured by this method.

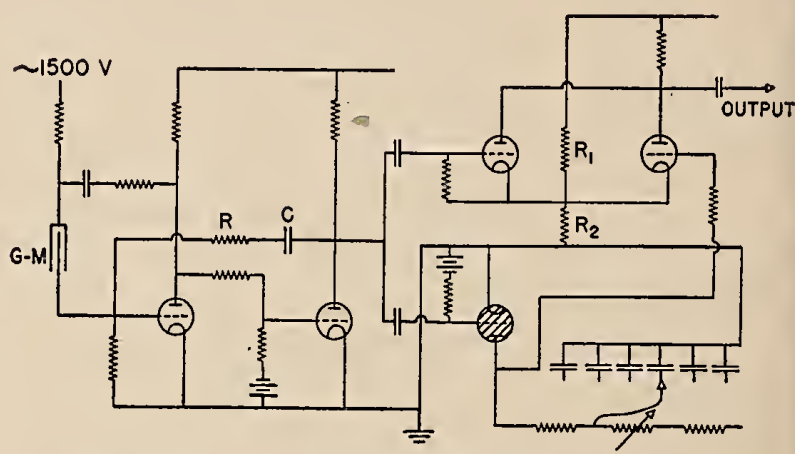

FIGURE 83. Wiring diagram of circuit used to measure half-value periods by delayed "coincidences."

The procedure for measuring half-periods is to determine $C$ for various values of $T$ and also the total counting rate, $\left(n a+2 n_{b}\right)$. From these measurements, and eq $96, \lambda$ can be determined. This method is easiest for values of $\lambda$ greater than $\left(n_{a}+n_{b}\right)$, with $n_{a}$ as small as possible. A diagram of the coincidence circuit used by Ward is shown in figure 83. The first two tubes are connected in a multivibrator circuit, which quenches the counter and delivers uniform pulses to the pair of condensers where the pulses are split, part going to the Rossi circuit in the upper portion, and part going to the grid of the thyratron.

A single pulse from the Geiger-Müller counter produces a pulse of negative voltage at the plate of the second vacuum tube. The length of this pulse is determined by the values of $C$ and $R$. The time cannot be made less than $10^{-4} \mathrm{sec}$, since this causes the multivibrator to trip more than once. The rectangular pulse, shown in figure 84 , a, is fed through the two capacitors to the second part of the circuit. This coupling has a small time constant, which modifies the 
negative pulse as shown by the dotted lines in figure 84 , a. The cathode potential of the two Rossi tubes is held about $100 \mathrm{v}$ positive with respect to ground by the potential divider $R_{1}$, $R_{2}$. The control grid of the second of these two tubes is connected through a megohm resist-
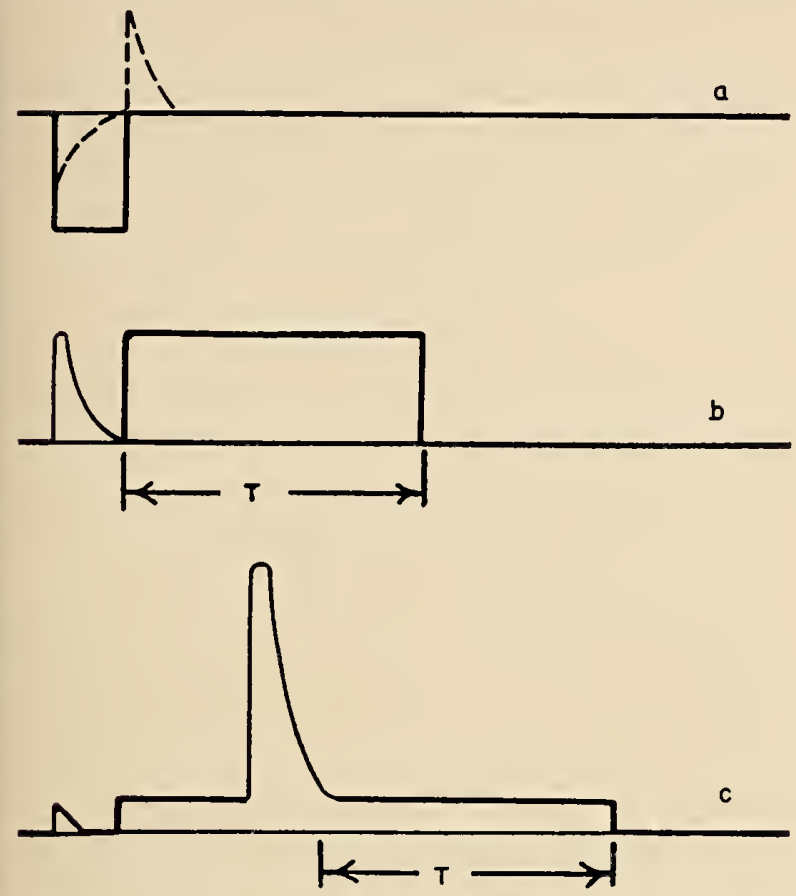

FIGURE 84. Diagram of voltage pulses at various points in the circuit shown in figure 83.

ance to the plate of the thyratron. The voltage pulse represented by the dotted line in figure 84 , a is fed to the grid of the thyratron and to the grid of the first Rossi tube. The voltage changes occurring across the plate resistor of the Rossi tubes is shown in figure $84, \mathrm{~b}$. The initial short pulse is caused by the blocking of the first Rossi tube by the negative part of the pulse arriving at the grid; and the second positive pulse of figure 84 , a causes the thyratron to strike, communicating a negative pulse to the second Rossi tube from the plate of the thyratron. This second tube remains nonconducting for a variable period, $T$, depending on the resistance and capacity associated with the plate of the thyratron that can be adjusted. The output voltage changes occurring at the plates of the Rossi tubes when two pulses occur within the time, $T$, are shown in figure 84 , c. The second pulse is recorded as a coincidence by the large change in voltage occurring at this point. Since the second tube of the Rossi pair will be held nonconducting for a time, $T$, after the second pulse, another coincidence will be recorded if an additional pulse occurs within this time. Thus the circuit records every pulse following a preceding pulse within a time, $T$.
Ward measured the half-value periods of $\mathrm{AcA}$, ThA, and $\mathrm{RaC}^{\prime}$ by this method, obtaining the values: $\mathrm{AcA}=(1.83 \pm 0.04) \times 10^{-3} \mathrm{sec} ; \mathrm{Th} A=$ $(1.58 \pm 0.08) \times 10^{-1} \mathrm{sec} ; \operatorname{RaC}^{\prime}=(1.48 \pm 0.06) \times$ $10^{-4}$ sec.

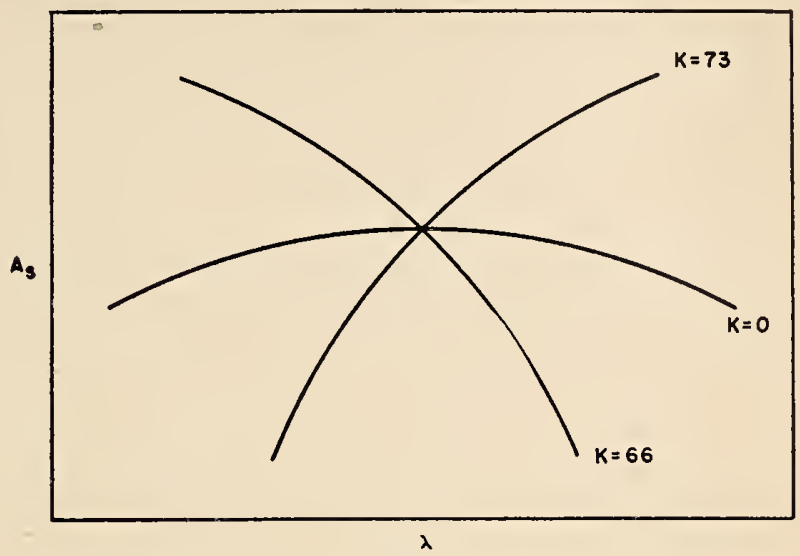

Figure 85. Curve showing variation of $\mathrm{A}_{\mathrm{s}}$ with $\lambda$ for. different values of $K$.

\section{Measurement of Half-Value Periods with Counters}

w'hen making measurements of half-value periods of the order of a few minutes to a few hours, using the straight-forward method of reccrding the counting rate at intervals, a great varjation in counting rates is encountered when, as is desirable, the activity is followed ove' several half-periods. This usually requires that; the faster rates be corrected for counting losses, a procedure that is time-consuming and difficult to do with precision. Graves and Walker [79] have suggested a method that can be used for neutron-activated isotopes, which elininates the correction for counting losses. The principle may have other applications.

Let $\mathrm{N}$ represent the total number of counts obtained by counting the activated sample $t_{2}$ minutes. From this measurement the saturated activity, defined as the initial counting rate of a sample irradiated for an infinite time, can be computed from the relation

$$
A_{s}=\frac{N e^{\lambda t_{3}}}{\left(1-e^{-\lambda t_{1}}\right)\left(1-e^{-\lambda t_{2}}\right)},
$$

where $t_{1}$ is the time during which the sample was irradiated, $t_{3}$ the time between the irradiation and the start of counting, and $\lambda$ is the decay constant of the activated isotope. Differentiating $A_{s}$ with respect to $\lambda$ gives

$$
\frac{d A_{s}}{d \lambda}=K A_{s},
$$


where

$$
K=1 / \lambda+t_{3}-\frac{t_{1} e^{-\lambda t_{1}}}{1-e^{-\lambda t_{1}}}-\frac{t_{2} e^{-\lambda t_{2}}}{1-e^{\lambda \mathrm{t}_{2}}} .
$$

Now if counting times are chosen to make $d A_{s} / d \lambda=K A_{s}=0$, the value of $A_{s}$ will not depend markedly on a small error in $\lambda$. Then if other times are selected for which this derivative is not zero, the new calculated value of $A_{s}$ will differ from the first value if an incorrect value of $\lambda$ has been assumed. Assuming that $A_{s}$ is an approximately linear function of $\lambda$, the true decay constant, and designating the provisionally selected value of $\lambda$ as $\lambda^{\prime}$, we can write

$$
\lambda=\lambda^{\prime}+\left(A_{s}-A_{s}^{\prime}\right) / K A_{s}^{\prime},
$$

where $A_{s}^{\prime}$ is the calculated activity from eq 20 by using $\lambda^{\prime}$.

For this method to be useful, enough counts must be recorded to make statistical errors small, $K$ must have predetermined values, and the counting rates for various sets of observations must be very nearly the same. These three conditions can be met, since there are also three parameters $t_{1}, t_{2}$, and $t_{3}$ that can be

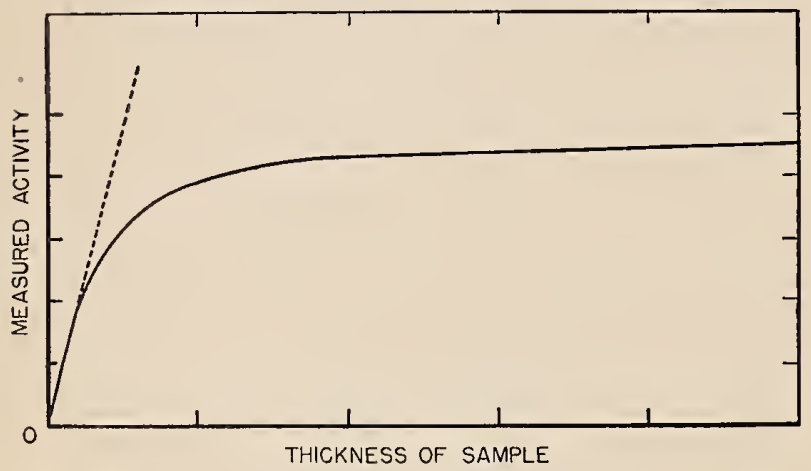

Figure 86. Curve showing typical increase of betaray activity with thickness of sample.

adjusted. Since all activated samples will have the same activity when counted, the counting loss correction will be the same for each and will not affect the value of $\lambda$, as computed from eq 101. From eq 100 , it can be seen that the sensitivity of the method, defined as the percentage difference in $A_{s}$ divided by the percentage error in $\lambda$, is equal to $K A_{s}$. If conditions are selected so that this product is equal to unity, then activities must be measured to 1 percent to detect an error in $\lambda$ of 1 percent. Figure 85 shows a schematic representation of $A_{s}$ as a function of $\lambda$ for three different values of $K$. Since $A_{s}$ cannot vary for these different values if the value of $\lambda$ is correct, all such curves must intersect in a common point, as shown. In practice, experimental errors will result in three nearby points of intersection and three values of $\lambda$. Applying eq 101 to any two of these curves and eliminating $A_{s}$, the following expression for $\lambda$ is obtained

$$
\lambda=\lambda^{\prime}+\frac{A_{\mathrm{s}}^{\prime \prime}-A_{\mathrm{s}}^{\prime}}{\left(K^{\prime} A_{\mathrm{s}}^{\prime}-K^{\prime \prime} A_{\mathrm{s}}^{\prime \prime}\right)},
$$

where $A_{\mathrm{s}}^{\prime \prime}$ and $A_{\mathrm{s}}^{\prime}$ are the saturated activities calculated, with $\lambda^{\prime}$ and counting schedules corresponding to $K^{\prime \prime}$ and $K^{\prime}$.

\section{Determination of Very Long Half-Periods}

The measurement of half-periods of the order of several years or greater is difficult by direct observation of the change of activity with time. Considerable care is required to maintain a uniform sensitivity of the measuring equipment. By using a source that has a much longer period than that of the isotope under investigation as a reference for frequent recalibration, satisfactory results can be obtained for periods of the order of $10 \mathrm{yr}$ or less if observations can be continued over an interval of one or more periods.

For periods exceeding $10 \mathrm{yr}$, the determination of the specific activity of the sample is a practicable method in many instances. This method is based on the fundamental expression

$$
\frac{d N}{d t}=-N \lambda
$$

where $d N / d t$ is the rate of disintegration of the sample, and $N$ is the number of radioactive atoms present at the time of observation. This method is complicated by the fact that it is not always easy to measure either $d N / d t$ or to know the precise value of $N$, both of which are required. In the case of isotopes emitting alpha particles it is relatively simple, in principle, to determine the rate of disintegration by counting the particles in a definite geometrical arrangement where the fraction of the total particles counted is known. If the half-period to be measured is greater than $10^{8} \mathrm{yr}$ and the isotope can be prepared with a high degree of chemical purity, the rate of emission of particles may be determined directly from weighable samples [80]. For shorter periods, samples for counting must be obtained by subdividing weighable amounts of the isotope of known chemical purity, until counting rates that can be recorded are obtained. The same method in principle can be applied to isotopes of long periods undergoing beta-ray decay. However, the determination of the disintegration rate in terms of the 
specific activity is much more difficult for substances of long life emitting beta rays. More sensitive detectors must be used than for alpha particles, and the effects of variation of the solid angle and of back scattering are less easily evaluated. The energies of the disintegration beta rays are spread over a continuous spectrum, and the interpretation of the results may be complicated by the presence of conversion electrons. Finally, the disintegration scheme must be known in order to apply this method.

\section{Half-Periods from True Coincidence Measurements}

A method has been suggested by Riedhammer [81] for using the conventional coincidence arrangement for the determination of half-value periods. When two counters (1 and 2 ) are connected in a coincidence arrangement the accidental coincidence rate, $C$, is given by the usual relation

$$
C=2 N_{1} N_{2} \tau,
$$

where $\tau$ is the resolving time, $N_{1}$ and $N_{2}$ are the individual counting rates of the two counters considered constant when averaged over a sufficiently long time, and the source for each counter is independent of the other.

If the sources irradiating the counters 1 and 2 are not constant but are two different samples of the same radioisotope, the values of $N_{1}$ and $N_{2}$ are no longer constant but are represented by $N_{1}=N_{0} e^{-\lambda t}$, and $N_{2}=N_{0}^{\prime} e^{-\lambda t}$, where $N_{0}$ and $N_{0}^{\prime}$ are the initial intensities at $t=0$. Introducing these expressions into the equation for $C$, we obtain

$$
C=2 \tau N_{0}^{\prime} N_{0} e^{-2 \lambda t}=C_{0} e^{-2 \lambda t} .
$$

For the half-period of the accidental coincidences, $T_{c}$, that is the period required for $C$ to become $C_{0} / 2$

$$
C=C_{0} / 2=C_{0} e^{-2 \lambda T c},
$$

or

$$
T_{c}=1 / 2(\ln 2 / \lambda)=1 / 2 T^{*},
$$

where $T^{*}$ represents the half-period of the radioisotope.

This method provides a very rapid method for determining half-value periods, since the decay curve for $T_{c}$ is much steeper than for $T^{*}$, which has particular advantages for the measurement of long periods. Ordinarily these require long periods of observation during which experimental conditions must be maintained constant.

\section{Statistical Error in Measurement of Half-Value Periods with Counters}

The limitations in accuracy imposed by the statistical fluctuations become important in determining the half-value periods of beta-ray emitters of relatively short period. A compromise between the desired number of counts for a particular point on the decay curve and the change of average activity during the observation must be made. Peierls [82] has indicated a method for obtaining an improvement in the statistical accuracy of such measurements with an economy in number of observations required compared with the usual simple methods.

The following simple methods for determining $\tau, \quad(\tau=1 / \lambda)$ are frequently used:

(1) The number of particles are counted during short periods, and their logarithm is plotted against the time. These observations should fall on a straight line with a slope equal to $\lambda$. This method is open to the objection that it is difficult to estimate graphically the error, and the slope of the line obtained in this way is strongly influenced by the observations taken near the end where the counting rate is low.

(2) The total number of counts, $n_{2}$, taken after a chosen time, $t$, is compared with the total number of counts, $N$, taken from $t=0$.

Then

$$
r=\frac{n_{2}}{N}=e^{-t / \tau} \text {, or } \tau=-\frac{t}{\ln r} .
$$

The relative error is a minimum for

$$
n_{2} / N=e^{-t / \tau}=0.2 \text {. }
$$

(3) Another method is to observe counts for equal intervals of length, $t$. Representing these counts as $n_{1}, n_{2}, n_{3} \ldots$, the following expression is evaluated

$$
r=\frac{n_{1}+n_{3}+\ldots}{n_{2}+n_{4}+\ldots} .
$$

The statistical average of $r$ is $e^{-t / \tau}$, or approximately

$$
\tau=\frac{t}{\ln r} \text {. }
$$

This method can be shown to give a somewhat higher minimum relative error and is therefore less efficient than method (2), although more complicated.

In a rigorous mathematical treatment Peierls shows that the best approximation to the mean life $\tau$ is the arithmetic mean of the individual times at which particles have been observed. 
This may be written $s=\Sigma t v / m$, where $m$ is the total number of particles, $t_{v}$ is the midpoint of the $\nu$ th interval, and $s=\tau$ for an infinite time of observation, $T$. When $T$ is finite

$$
-s=\frac{T}{e^{T / \tau-1}},
$$

from which a correction $\sigma$ to the computed $\tau$ may be calculated for various values of $T / \tau$.

Assuming that values of $T / \tau, T / \mathrm{s}, \tau / s$, and $\sigma$ are available, the procedure for determining $\tau$ is to divide the total time available, $T$, into intervals of length $d<0.3 \tau$. In practical cases where this results in a small number of counts, larger intervals may be taken. The number of counts for each interval is determined, represented by $n_{1}, n_{2}, \ldots$ and their sum by $m$. The expression

$$
s_{0}=\left(n_{1}+3 n_{2}+5 n_{3}+\ldots\right) \frac{d}{2 m},
$$

is taken as a first approximation for $s$. From the corresponding values of $T / s$, the value of $\tau / s_{0}$ can be ascertained, which gives an approximate value for $\tau$. For a more accurate value, a second approximation for $s$ can be obtained from the relation

$$
s_{1}=-1 / 12 \frac{d^{2}}{\tau},
$$

and the corresponding correction for $\tau($ tau $)$ will give

$$
\tau_{1}=\sigma s_{1}
$$

The most efficient value for $T$ is computed as approximately $4 \tau$.

TABLE 75.-Values of $T / s, \tau / s$, and $\sigma$ as computed by Peierls for various values of $T / \tau$.

\begin{tabular}{|c|c|c|c|}
\hline$T / \tau$ & $T / s$ & $r / s$ & $\sigma$ \\
\hline 1.0 & 2.39 & 2.392 & 12.5 \\
1.2 & 2.48 & 2.071 & 9.09 \\
1.4 & 2.58 & 1.846 & 6.71 \\
1.6 & 2.69 & 1.680 & 5.29 \\
1.8 & 2.80 & 1.554 & 4.31 \\
2.0 & 2.91 & 1.456 & 3.63 \\
2.2 & 3.03 & 1.378 & 3.12 \\
2.4 & 3.16 & 1.315 & 2.72 \\
2.6 & 3.29 & 1.263 & 2.17 \\
2.8 & 3.42 & 1.221 & 1.98 \\
3.0 & 3.56 & 1.186 & 1.65 \\
3.5 & 3.93 & 1.122 & 1.44 \\
4.0 & 4.32 & 1.081 & 1.30 \\
4.5 & 4.74 & 1.053 & 1.10 \\
5.0 & 5.18 & & 1.05 \\
6 & 6.09 & 1.015 & 1.02 \\
7 & 7.04 & 1.006 & \\
8 & 8.02 & &
\end{tabular}

\section{RADIOACTIVE TRACERS}

The use of radioactivity to identify individual atoms in a chemical compound is relatively old. For example, v. Hevesy and Paneth [83] investigated the solubility of lead sulphides and lead chromates using $\mathrm{RaD}$ as a tracer, in a paper published in 1913. In this case the tracer was used merely to extend the sensitivity of existing methods of measurement. At that time the lower limit for a qualitative test for the presence of lead using the most sensitive microchemical reaction was $3 \times 10^{-9} \mathrm{~g}$, and the quantitative limit was considerably higher. By using $\mathrm{RaD}$ as an indicator they were easily able to measure $10^{-10} \mathrm{~g}$ of lead. If time is given for the RaE to come into equilibrium this measurement could be carried to $10^{-12} \mathrm{~g}$ of RaD.

An example of a typical tracer problem in which the identification of particular groups of atoms is used is provided by the investigation by v. Hevesy [84] of the exchange of atoms between liquid and solid phases. Since only naturally radioactive isotopes were available the method was limited to a few cases where radioactive isotopes of common stable elements were available. Thus v. Hevesy investigated the exchange of atoms between metallic lead and a solution of lead nitrate using ThB mixed with the lead in one phase. He was able to show that the exchange of lead atoms between the metal and solution was very rapid, arising chiefly from local action and that the lead atoms entered the solution at individual points on the metal and were redeposited at other points. The tracer method is practically the only way to obtain this type of information reliably and accurately.

Now that more than 400 radioactive isotopes are available, the tracer method can be extended to a great variety of problems, in physics, chemistry, biology, and industrial applications. In practice the number of radioisotopes that can be used for tracer methods is limited by the individual characteristics of these isotopes. For example, a reasonably long half-period, radiation of sufficient energy to be readily measured, and a convenient supply of the isotope in suitable form are obviously desirable. 


\section{Preparation and Measurement of Tracer Samples}

It is fortunate that for most tracer applications absolute values of the activity of the samples are not required, and satisfactory results can be obtained by measuring activities relative to an arbitrary standard. All measurements must then be made with samples in fixed positions relative to the detecting instrument. Furthermore, since tracer samples taken from the material under investigation are of low specific activity, consideration must be given to the form in which samples are compared. In thick samples compared by means of the beta radiation, a common and sensitive procedure, part of the radiation emitted in the direction of the detecting devices is absorbed. Also, some radiation not originally directed to the detector is scattered in the sample in a way that causes it to be measured. Therefore each sample should have the same shape and composition for accurate comparison. In certain cases approxima-

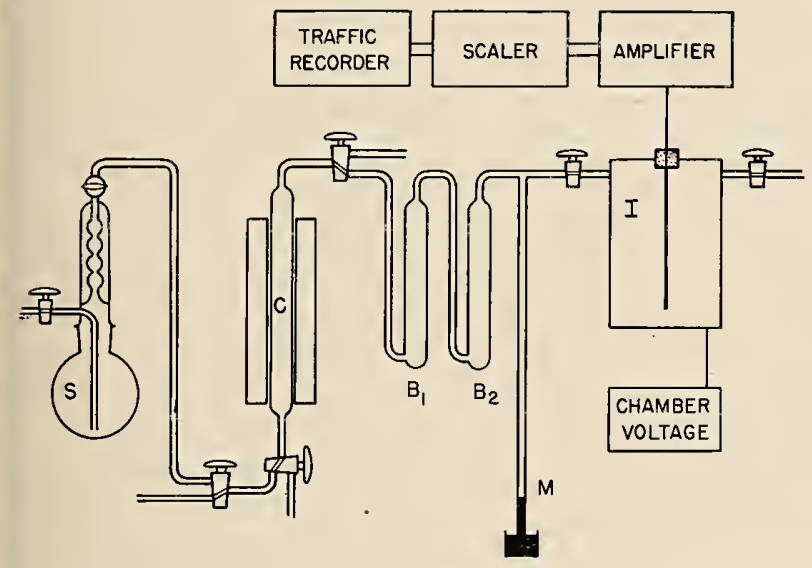

FIGURE 87. Apparatus for measuring small quantities of radium in terms of radon.

$S=$ standard radium solution; $C=$ reduced copper in furnace; $B_{1} B_{2}=$ drying bulbs; $M=$ mercury manometer; $I=$ ion-counting chamber.

tions to the ideal condition are adequate. For example, samples that are thick enough to absorb completely the radiation from the layers farthest from the detector need only have identical geometrical surfaces. The thickness required, expressed in grams per square centimeter is determined by the maximum range of the beta rays from the radioisotope that is used. For $\mathrm{C}^{14}$ this thickness need only be of the order of 15 to $20 \mathrm{mg} / \mathrm{cm}^{2}$.

When identical conditions for all samples and the working standard cannot be realized for various reasons, such as limited amounts of material, then corrections must be made for selfabsorption. These corrections are best determined by measuring material with a given con- centration in layers of increasing thickness. The general features of such a calibration are shown in figure 86 where grams per square centimeter of sample is plotted against the measured activity. As the first layers are added, the activity increases almost linearly with the thickness." As the effect of self-absorption becomes more prominent the activity measured falls below the initial straight line and finally, when all radiation from the bottom layer is absorbed in the sample, additional layers cause no increase in the measured activity.

\section{Absolute Measurements of Tracer Samples}

Where absolute measurements of the activities of tracer samples are desired in rutherfords, or disintegrations per second, comparisons must be made with calibrated standards. In general the standards must be prepared from the same radioisotope as the tracer, and both standard and sample must be in the same physical form. When reference standards calibrated by the National Bureau of Standards are available, working standards to match the tracer samples in form, may be prepared by comparing them with the reference standard.

Radioactive tracers may be useful in two general ways. When they are mixed with their inactive isotope in a definite ratio that is maintained throughout all subsequent operations, they serve to trace the element under investigation. This tracing may be made quantitative. However, under certain conditions interchange of atoms of the element under investigation with similar atoms in another compound or phase may occur. Therefore, the ratio of active to inactive atoms changes. Although the tracer method can be used to study such exchanges, the presence of this interchange may interfere with quantitative results by the tracer method. This difficulty can be obviated by use of compounds in which the atoms of the element under investigation are tightly bound.

In the field of physical chemistry, tracer methods have been used to determine solubilities of compounds of low solubility, to measure the absolute magnitude of surfaces of crystalline precipitates, to study absorption and coprecipitation of ions.

In engineering [85] the effect of lubrication on friction has been studied by determining by radioactive measurements the amounts of metal removed from inactive slider moving over an active surface. Radioactive methods have been used to measure the rate of movement of water through the flumes of electrical generating plants.

The availability of $\mathrm{C}^{14}$ in large amounts has stimulated the use of tracer methods in biology, physiology, and medicine. By such methods the 
behavior of many organic compounds in living organisms can be studied in detail quite impossible without this aid. The movement of substances labelled with radioactive carbon can be followed and quantitative determinations made at various intervals to determine not only the location but rate of arrival of the substances. Similarly, such elements as phosphorus, sodium, boron, and iodine, to mention a few, which play important roles in animal and vegetable life are finding increasing use as biological tracers.

\section{RADIOACTIVE STANDARDS AND UNITS}

Much of the development of the subject of radioactivity has resulted from relative measurements expressed in arbitrary units.

When practical applications are made of radioactive isotopes, common standards of comparison become necessary. This is well illustrated by the history of the discovery and use of radium. As long as radium and its decay products remained a tool of physicists engaged in studying the phenomenon of radioactivity, very little need was felt for standards. About the only standardization attempted was for each laboratory engaged in radioactive investigations to select a preparation of radium in its possession as a laboratory standard to which all measurements in that particular laboratory were referred. Frequently these preparations of radium were of unknown and doubtful purity and the exact amount of radium present undetermined.

Under these circumstances all measurements based on quantitative knowledge of the amount of radium used were subject to widely conflicting variations. The situation was further intensified when practical use for radium in treatment of malignancies developed and radium became an article of commerce. Therefore, in 1910 an International Radium Commission was established to prepare radium standards. These standards were weighed out from radium used by Mme Curie in the determination of the atomic weight of radium. These were intercompared by gamma-ray methods and distributed to various nations as primary national standards, one being retained at Sevres as an international standard.

The development of artificial radioactivity has repeated the cycle of events illustrated by radium. Recently the need for standards in the field of radioactive isotopes has been emphasized by a divergence of the order of 300 percent among laboratories measuring such isotopes as $\mathrm{P}^{32}$ and $\mathrm{I}^{131}$ for clinical use. The provision of standards for induced activities is made difficult by their relatively short halfvalue periods in most instances. Since radioactive standards preferably should be prepared for each isotope, the problem assumes considerable proportions. The National Bureau of Standards has undertaken to provide standards of those isotopes that are suitable and can be calibrated in absolute disintegration rates. For those of short half-value periods, a system of distribution of standard samples is under consideration.

\section{Alpha-Ray Standards}

Uranium has long been used for the calibration of alpha-ray electroscopes and ionization chambers. The advantage of a long life and consequent use of weighable quantities is partly offset by the self-absorption of the particles in thicker layers. When used in connection with ion chambers and amplifiers arranged to count the individual particles, uranium can be used in thin electrolytic deposits where the selfabsorption is slight. Plutonium will probably prove to be superior to uranium since it emits particles of a single range and has a much higher disintegration rate.

\section{Beta-Ray Standards}

Standards emitting beta rays are certain to prove most useful in the measurement of radioactive isotopes where beta and positron disintegrations are most common. Comparatively few of the artificial isotopes appear to be adapted to the preparation of absolute standards. For an absolute standard, the disintegration scheme must be known and be preferably simple; that is, consist of the emission of a beta ray followed by one or more gamma rays. For ideal conditions, conversion electrons must be absent. However if the details of the disintegration scheme are accurately known, more complex modes of disintegration can be dealt with, including the presence of conversion electrons.

The most promising isotopes at the moment are $\mathrm{Co}^{60}$ and $\mathrm{Na}^{22}$. As more is known regarding the disintegration schemes of other isotopes of reasonable half-life, this list may grow.

There are two general ways in which beta ray standards may be prepared. One is to prepare a deposit having a known disintegration rate. This standard may then be used to calibrate a beta-ray counter. For the bell-type mica window counters this standard should have a 
circular deposit of smaller diameter than that of the counter window. Back-scattering will play an important role in the use of such a standard. The most satisfactory arrangement is to deposit the activity on a thick support that yields saturation back-scattering. If all samples compared with this standard are similarly mounted, the effect of the back-scattering on the comparison is eliminated.

A second method of providing beta-ray standards is to prepare a solution of the isotope with a known disintegration rate per milliliter. The user can then prepare deposits of known activity to suit his individual needs. This method relies on the experience and skill of the user but does provide for more flexible use.

A variation of the solution standard that provides both a beta- and gamma-ray standard is to prepare a sealed ampoule containing a solution of the standardized isotope. This ampoule, the contents of which are never disturbed, may be used to prepare beta-ray working standards. The gamma radiation from a solution of the same isotope in a volume similar to that of the standard is compared by a gamma-ray instrument with that from the standard. This gives the data for computing the disintegration rate in the unknown solution. Appropriate deposits of known disintegration rates may now be prepared from the calibrated solution.

Where the amount of an isotope for the preparation of a standard is limited, it is preferable to prepare deposits. This situation is illustrated by the $\mathrm{RaD}+\mathrm{E}$ standards prepared by the National Bureau of Standards. To prepare them, the lead is quantitatively removed from a carefully weighed specimen of high-grade pitchblende. The amount of radium per gram of pitchblende is determined by the radon method used for determination of radium in ores and rocks. From the radium content and the total amount of lead, the number of disintegrating $\mathrm{RaD}$ atoms per unit time in the sample of lead can be determined, and the corresponding number per milligram of lead computed. Standards are prepared by depositing the lead in the form of $\mathrm{PbO}_{2}$ by electrolysis on palladium foil backed by $1 / 16$ in. of silver. In a typical preparation, $1 \mathrm{mg}$ of deposited lead corresponded to 138 disintegrations per second. The $\mathrm{RaE}$ comes into equilibrium in approximately 30 days and then the standard decays with the half-value period of $\operatorname{RaD}(22.2 \mathrm{yrs})$. Polonium gradually comes into equilibrium in these standards. Therefore, in use, the alpha particles from polonium must be screened out in those cases where the counter window is thin enough to transmit them. When the polonium is in equilibrium, which requires about 3 yrs, the acuracy of these standards can be rechecked by counting the alpha particles with suitable corrections for self-absorption and scattering. Stand- ards prepared by this procedure can be expected to be accurate within \pm 5 percent. The chief uncertainty is in the assumed value of the curie used to compute the rate of disintegration of the measured amount of radium in the pitchblende.

Since the shape of the continuous beta-ray spectrum of an isotope influences considerably the percentage of the spectrum transmitted through a counter window, a beta-ray standard prepared from one isotope cannot be used for calibrating a counter to measure another isotope, even though the maximum energies of the beta rays are nearly the same. In extreme cases, such a procedure may result in errors of 40 percent or more. Therefore, beta-ray standards are, in general, required for every isotope that can be measured in disintegrations per second.

It is to be noted that isotopes that emit only beta rays cannot be calibrated by the coincidence method. In view of the restrictions on the measurement of isotopes by use of standards prepared from other isotopes, it is difficult to measure isotopes that emit only beta rays in disintegrations per second. Attempts have been made to measure this class of betaray emitters in terms of an absolute geometry. For relatively strong preparations, this method may be developed to provide satisfactory measurements particularly for beta rays that have fairly high energies. This method, for best results, requires the use of thin windows, a geometry for which the solid angle can be computed, and an experimental alrangement in which scattering into the aperture of the counter is reduced to a negligible amount. Finally, in general, corrections are required for the back-scattering from the support and for self-absorption in the source.

\section{Gomma-Ray Standards}

For calibrating counters, electroscopes, and ionization chambers, radium gamma-ray standards, containing suitable amounts of radium, are useful. They can be used quantitatively only for calibrating instruments to measure radium, however. Furthermore, all beta rays should be screened out for reliable gamma-ray comparisons. The equivalent of 1/8-in. of lead is sufficient for this purpose. Uniform radium gammaray standards can be used in checking the linearity of the gamma-ray response of gammaray counters. The National Bureau of Standards has available for distribution radium gamma-ray standards consisting of ampoules with $5 \mathrm{ml}$ of radium solution containing 0.1 , $0.2,0.5,1.0,2.0,5.0,10.0,20.0,50.0$, and 100 $\mu \mathrm{g}$ of radium.

Gamma-ray standards of artificially active isotopes are required for calibrating devices for gamma-ray measurements of these isotopes 
in absolute disintegrations per second. As in the case of beta-ray standards, few radioactive isotopes are suitable for absolute standards. Various compromises can be made to secure approximate calibrations of gamma-ray instruments. A series of several isotopes having suitable disintegration schemes, including approximately monochromatic gamma rays, may be used to provide standards with a range of gamma-ray energies. This series of standards may be used to determine the sensitivity of the detector for the various energies of the gammaray standards. By using a calibration of this type, other isotopes with known gamma-ray energies and disintegration schemes can be measured approximately in terms of disintegration rates, provided the disintegration scheme shows that each disintegration is accompanied by gamma radiation.

\section{Neutron Standards}

At present, no very satisfactory neutron standards exist. The earlier standards consisted of radium mixed with beryllium in a sealed capsule. There is much evidence [86] to show that such preparations vary in relative efficiency of production of neutrons, depending on the details of the method of preparation. Therefore the rate of emission of neutrons cannot be predicted from a knowledge of the radium content and the ratio of beryllium to radium. These preparations have other serious disadvantages that impair their usefulness, such as lack of knowledge of the relative distribution of energies of the neutrons.

Gamertsfelder and Goldhaber [87] have suggested photo neutron sources to obviate the difficulties of reproducibility encountered with mixtures of radium and beryllium. They suggest that irradiating a definite amount of beryllium with the gamma rays from radium in a well-defined geometry should give a reproduceable standard.

They gave consideration to a geometry in which the dimensions of the radium source do not play an important role to permit standards to be constructed by using existing preparations of radium. For an experimental trial, they used a source consisting of $100 \mathrm{mg}$ of radium in a monel tube of $1-\mathrm{mm}$ wall thickness, $4.85-\mathrm{mm}$ external diameter and 2-cm length. This source was used to irradiate a cylindrical block of beryllium of 1.5-in. diameter and height. The center of the source was placed $4 \mathrm{~cm}$ above the upper surface of the beryllium with the axis of the source parallel to the upper surface of the beryllium. The absolute number of neutrons emitted by this source was determined by slowing all neutrons to thermal velocity. Then the ratio, $R$, of the number of neutrons activating a $\mathrm{MnSO}_{4}$ solution, with and without an absorber, was determined by using a volume of solution large enough that only 1 percent of the neutrons escaped. Then the number of neutrons, $N_{a}$ captured by the absorber per unit time was determined by determining the activity of the manganese powder used as an absorber. The total number of neutrons emitted per unit time is given by

$$
N=\frac{N_{a}}{1-R} .
$$

For the source described above this gave $N=(8.6 \pm 0.8) \times 10^{4}$ neutrons per second. Such methods of calibration give an estimate of the number of neutrons emitted per unit time but do not, of course, reveal the original distribution of energies.

The need for neutron standards will increase with time, and it may be expected that more suitable standards will be developed.

\section{Units Used in Radioactive Measurements}

Curie unit.-When radium was the principal radioactive element in general use, two welldefined units met all requirements for quantitative measurement. One was simply $1 \mathrm{~g}$ of radium, that could be determined from gravimetric weighing. Since preparations of radium commonly were used in amounts of milligrams, this unit of weight was employed in practice. Amounts of radium are determined by comparing the gamma radiation from an unknown sample with that of a carefully weighed standard under the same conditions of filtration, by using the same instrument for detection of the gamma radiation. In many cases radon is used in the place of radium, since all the products emitting important gamma rays in terms of intensity and energy occur subsequent to radon in the decay chain: Therefore, the name curie was applied to that quantity of radon in equilibrium with $1 \mathrm{~g}$ of radium. Again quantities of radon can be measured in curies by comparison of the gamma radiation with that from a radium standard. Subsequently, it was recognized that the curie also could be applied to any equilibrium quantity of the decay products of radium, and this was done by specific recommendation of the International Radium Commission. Although it is recognized that the curie also refers to a disintegration rate, namely the number of atoms disintegrating per unit time in $1 \mathrm{~g}$ of radium or any of its products in equilibrium, it has never been possible to determine this number better than \pm 5 percent. 
In extending the use of the curie to other members of the radium family, the International Radium Commission [88] definitely opposed its use outside this family. In other words, it cannot be used correctly in referring to amount of various members of the actinium or thorium families. In spite of this published opposition, it became quite common in the decade following this pronouncement for workers to express quantities of artificially radioactive isotopes in curies, frequently when they could not possibly measure the quantities in disintegrations per unit time, which would be the only condition under which such unofficial use might be logical. For those isotopes that do not emit alpha rays, it is very difficult to measure the disintegration rate. For beta-ray and positron emitters where it is known that one particle is emitted per disintegration, a beta-ray counter or other detector must be calibrated in absolute over-all efficiency for the radiation emitted by the isotope in question before the disintegration rate can be determined reliably. Such disintegration schemes are known for a relatively small fraction of known radioisotopes. It is obvious then that the measurement of isotopes in terms of disintegration rates is restricted. Therefore, the unofficial use of the curie has been even more unfortunate than a mere misinterpretation of the definition and restrictions on its use.

It may be well to point out here another patently erroneous use of the curie unit for which there is no logical justification. In the use of radon for therapy, the curie has been used loosely to refer to the gamma radiation from a curie of radon. As long as this use was confined to radium or radon no serious error was involved. However, this misuse leads to considerable error when attempts are made to compare the gamma radiation from any other radioactive isotope with that from radium or radon. Since nearly all detection instruments give widely differing indications for gamma rays of different energies, the equality of response of a given instrument by no means represents equal intensities of gamma rays from different isotopes. When, as has been done frequently, this misuse is extended to imply that equal amounts of the isotopes are present under these circumstances, utter confusion results, particularly when measurements are made in different laboratories with different instruments. Therefore, the use of the curie to express either the intensity of the gamma radiation or the relative amount of the isotope present in a source must be discarded.

Rutherford.-In those cases where disintegration rates can be measured, a unit has been proposed by Condon and Curtiss [89] with the suggestion that it be named after Lord Rutherford. The rutherford (abbreviation $\mathrm{rd}$ ) is de- fined as that quantity of any radioisotope that disintegrates at the rate of $10^{6}$ disintegrations per second. The purpose in proposing this unit was to eliminate the necessity for the unofficial use of the curie, with the attendant confusion, and to provide a unit that refers directly to the disintegration rate for any radioisotope. Since this unit can only be used in cases where the disintegration rate can be measured, the proper use of the unit automatically confirms that an actual disintegration rate is under discussion.

The magnitude of the rutherford was selected after considerable discussion with various workers in several countries. When applied to members of the radium family, 1 rutherford is 1/37 millicurie (assuming a nominal value of $3.7 \times 10^{10}$ disintegrations per second for the curie). The usual prefixes may be applied to the rutherford giving the following series:

Megarutherford ( $\mathrm{Mrd})=10^{6} \mathrm{rd}$, or $10^{12}$ disintegrations per second; Kilorutherford $(\mathrm{Krd})=10^{3} \mathrm{rd}$, or $10^{9}$ disintegrations per second; Millirutherford (Mrd) $=10^{-3} \mathrm{rd}$, or $10^{3}$ disintegrations per second, and Microrutherford $\left(\mu \mathrm{r}^{\mathrm{d}}\right)=10^{-6} \mathrm{rd}$, or 1 disintegration per second.

Thus, the microrutherford can be regarded as the basic unit. However, it should be noted that the majority of sources used in the laboratory will be expressed directly in rutherfords, with the millirutherford and microrutherford finding somewhat less frequent use. For example, the present therapeutic dose of $\mathrm{P}^{32}$ is of the order of $100 \mathrm{rd}$, and tracer quantities of most radioelements are of the order of 0.1 to $10 \mathrm{rd}$. Very rarely will a unit smaller than the microrutherford be required, since this corresponds to 60 disintegrations per minute, which is about the limit of reasonably accurate measurement with the more sensitive detectors.

Roentgen and roentgen per hour at a meter $(\mathrm{rhm})$.- There is considerable need for a unit of measurement for radioisotopes for which the disintegration scheme is either unknown or is of a type that does not readily permit measurements of disintegration rates. For those radioisotopes in this group that emit gamma rays, a convenient unit can be derived from the roentgen.

The roentgen $(r)$ is defined as "that quantity of X-or gamma-radiation such that the associated corpuscular emission per $0.001293 \mathrm{~g}$ of air, produces in air, ions carrying 1 esu of electricity of either sign." Since 1 esu represents $2.08 \times 10^{9}$ ion pairs, the $\mathrm{r}$ corresponds to $1.61 \times$ $10^{12}$ ion pairs per gram of air. Assuming an average value of $32.5 \mathrm{ev}$ for the production of a pair of ions in air, the roentgen corresponds to the absorption of $5.24 \times 10^{13}$ ev or approximately 83 ergs ( 1 ev $=1.60 \times 10^{-12}$ erg) per 
gram of air. Therefore, it is to be noted that the roentgen is defined in terms of the energy absorbed from the radiation by a selected quantity of air. From this definition it is apparent that the roentgen does not describe the radiation from which this energy is derived in respect to any of the basic characteristics of the radiation, such as energy of the photons comprising the radiation or the number of such photons. Consequently it is neither a measure of the energy per photon, sometimes referred to as "quality" or the intensity of the radiation in definite physical terms. Since its definition does not specify the time required to produce the specified number of ions, the roentgen does not measure a rate of delivery of energy that must be expressed as roentgens per unit time. In view of what has been stated above, it is obvious that the $r$ per second is a dosage rate, that is, a measure of the rate of delivery of energy, and not a measure of the intensity of the radiation, an error that is frequently made.

Roentgen per hour at 1 meter.-When the true nature of the roentgen is kept clearly in view, it provides a means for describing the relative amount of activity in a sample of any radioisotope emitting gamma rays. The first requirement is an instrument that measures roentgens reliably for radiations of various energies and in terms of the definition of the unit. Then the relative strength of the source is given by measuring the roentgens per unit time at a selected distance from the source. This determination yields a figure proportional to the activity permitting uniform relative comparisons of activity of any quantity of an isotope. A convenient unit for this determination is the roentgen per hour at $1 \mathrm{~m}$, abbreviated $\mathrm{rhm}$. With the restrictions indicated, $1 \mathrm{rhm}$ of any particular isotope will represent the same activity whenever measured. From determinations of the roentgens per unit time produced by $1 \mathrm{~g}$ of radium at a selected distance, it can be computed that $1 \mathrm{~g}$ of radium or 1 curie of radon is equal, within the accuracy of such measurements, to $0.83 \mathrm{rhm}$. Similarly, for $\mathrm{Co}^{60}$, for which the disintegration scheme is relatively well established, it can be estimated that $1 \mathrm{rd}=0.036 \mathrm{rhm}$.

Roentgen-equivalent-physical (rep). - In dealing with problems of protection of personnel, need has arisen for a unit, equivalent to the roentgen, that may be used in connection with other types of radiation that X-or gammarays for which the roentgen is defined. This unit should express the energy absorbed from these other radiations, such as beta rays, protons, alpha rays, and neutrons, by $1 \mathrm{~g}$ of tissue in the body. This conforms to the fairly well established general principle that bodily injury is proportional to the energy absorbed. It has been noted that 1 roentgen corresponds to approximately $83 \mathrm{ergs}$ per gram of air. Therefore, it is logical to define a unit for other types of radiation in terms of this amount of energy. This unit is the roentgen-equivalent-physical, rep, and is defined as that radiation which delivers 83 ergs per gram of tissue.

$n$ units.-Fast neutrons produce ionization in body tissues by means of the hydrogen recoil nuclei, which are released by impact of the neutrons on hydrogen in the tissues. Therefore, tissue damage in terms of energy absorbed in the tissues from fast neutron bombardment must be estimated from some convenient method of measuring the ionization produced by these neutrons.

A method of measurement that has ${ }^{\circ}$ proved convenient is to use an ionization chamber with Bakelite wall of the type represented by the Victoreen 100-r chamber. Fast neutrons drive hydrogen nuclei from the Bakelite, producing the measured ionization. When fast neutrons produce ionization equal to $1 \mathrm{r}$, as measured by chambers of this type, it has become customary to refer to this as 1 of neutrons. Evidence exists to show, however, that ionization of the body is approximately 2.5 times as great as that produced in a Bakelite chamber. Therefore, $1 n$ is equivalent to 2.5 rep, or about 200 ergs per gram of tissue.

Roentgen-equivalent-biological, (rem)-The whole story of radiation damage by such agencies as alpha particles or fast neutrons is not revealed when an estimate of the ionization in the tissue has been made. Biological evidence shows that the density of the ionization along the path of the ionizing agent has a great deal to do with the biological damage. For example, the energy absorbed from hydrogen recoil nuclei produced by fast neutrons is about five times as effective in producing injury to tissue as the same amount of energy absorbed from gamma radiation. Therefore, a unit that measures the biological effectiveness of various types of radiation has sometimes been employed. A commonly used example is the rem, used on the Manhattan Project. This abbreviation stands for radiation-equivalent-biological, the abbreviation $m$ being used instead of $b$ to avoid phonetic confusion with rep.

Therefore, $1 \mathrm{rem}$ is the amount of energy absorbed in tissue that produces the same biological effect as $1 \mathrm{r}$ of gamma radiation or $\mathrm{X}$-rays.

An estimate of the commonly accepted variations in biological effects from different radiations can be made from the value currently assigned to the $1 \mathrm{rem}$.

gamma and beta rays: 1 rep $=1$ rem alpha rays:

fast neutrons :

$0.1 \mathrm{rep}=1$ rem

.2 rep $=1$ rem 


\section{RADIOACTIVITY IN GEOLOGY}

One of the principal applications of radioactivity in geology has been the measurement of the age of various rock formations in the earth's crust. Such measurements are based on a knowledge of the decay constant of the natural radioactive isotopes and the ratio of these isotopes as they exist in nature.

\section{Uranium Lead Ratio}

A simple example is furnished by the uranium series. If it can be assumed that the uranium in a specimen of mineral containing this element was entirely separated from lead of radioactive origin at the time the mineral was formed, a measurement of the ratio $\mathrm{RaG} / \mathrm{U}$ in the mineral will give the time in which uranium has existed undisturbed in this specimen. One gram of $U^{238}$ with a decay constant $\lambda=1.52 \times$ $10^{-10}$ per year, produces approximately $1.32 \times$ $10^{-10} \mathrm{~g}$ of lead, RaG, per year. The interpretation of the $\mathrm{RaG} / \mathrm{U}$ ratio as measured in the minerals in terms of the time must take into account the decrease in the original amount of uranium during this period. An approximate correction is obtained by use of the ratio $\mathrm{RaG} / \mathrm{U}+0.58 \mathrm{RaG}$. This multiplied by $7.5 \times 10^{\circ}$ gives the age in years. It is necessary to know what fraction of the lead found in uranium minerals is RaG to apply this method. Ages of from 100 to 2,000 million years have been estimated by measurements of this type.

\section{Helium Content}

Measurement of the amount of helium in rocks that contain uranium and thorium in small quantities is a practicable method of estimating their age. This method is not limited to minerals rich in uranium as is true of measurements based on the amount of lead. In going from uranium to lead, eight alpha particles are emitted. If these remain in the rock until driven out by fusing in the laboratory, $1 \mathrm{~g}$ of uranium produces about $1.18 \times 10^{-7} \mathrm{cc}$ of helium per year. It is customary to determine the uranium content by measuring the radium and computing the equilibrium amount of uranium that is of the order of $3 \times 10^{6} \mathrm{~g}$ of uranium for $1 \mathrm{~g}$ of radium. Therefore, the age may be expressed in terms of the radium and helium and expressed in years, it is $\mathrm{He} / \mathrm{Ra} \times 2.8$, which also requires a correction for the decrease in the amount of uranium.

With both thorium and uranium present, as is the case for ordinary rocks where the helium method is most useful, a formula has been given by Hevesy and Paneth [90] for the computation of the age in years, as follows:

$$
\text { Age }=\frac{\mathrm{He}(\mathrm{cc})}{0.35 \mathrm{Ra}(\mathrm{g}) 3.0 \times 10^{-8} \mathrm{Th}(\mathrm{g})} .
$$

Whereas many radioactive minerals are unsuited for age determinations by the helium method because the structure of the minerals permits the escape of helium and the probability of loss is greater for the relatively large amounts of helium, the method does have advantages for ordinary rocks. First, the method of detecting and measuring helium is sufficiently sensitive and reliable to permit quantitative measurements for rocks containing as little as $10^{-13} \mathrm{~g}$ of radium per gram of rock. Secondly, rocks can be selected for measurement which have a structure which pretty well precludes the loss of helium by diffusion.

\section{Age of Terrestial Matter}

Koczy [91] has called attention to the computation by Stefan Meyer of the age of terrestial matter. Assuming that radioactive disintegration began when matter took its present form, Meyer [92] calculated the length of time required for producing all the uranium and actinium lead in existence through the disintegration of uranium. Koczy has recomputed this time by using the data provided by the massspectrometer measurements of Nier on lead and uranium. The values used are the isotope ratios of $\mathrm{Pb}^{204} ; \mathrm{Pb}^{206} ; \mathrm{Pb}^{207}=1.5 ; 22.6 ; 22.6$ the decay constant of $\mathrm{U}^{235}=9.72 \times 10^{-10}$ year ${ }^{-1}$, and the isotope ratio $\mathrm{U}^{238} / \mathrm{U}^{235}=\beta=139: 1$.

From the law of radioactive decay we have for uranium

$$
\lambda_{u} t_{a}=\ln \frac{U_{a}}{U_{0}},
$$

where $U_{a}$ is the number of uranium atoms present $t_{a}$ years ago, and $U_{0}$ is their present number.

Also

$$
U_{a}=U_{0}+a_{u} \mathbf{\Sigma} \mathrm{Pb}
$$

where $\mathrm{sPb}$ is the total lead produced by decay and $a_{n}$ is the ratio $\mathrm{Pb}^{206} / \mathrm{\textrm {Pb }}=0.226$. Combining these two equations gives

$$
t_{a}=1 / \lambda_{u} \ln \left(1+a_{u} \frac{\mathrm{\Sigma} \mathrm{Pb}}{\mathrm{U}_{0}}\right),
$$

with a similar equation for actinium lead. A graphical solution gives $t_{\mathrm{a}}$ as $5.3 \times 10^{9} \mathrm{yr}$. 


\section{Determination of Radium and Thorium in Rocks}

A convenient method for determination of radium in rocks consists in extracting the radon and measuring it with reference to a dilute radium standard. Standards containing $10^{-9} \mathrm{~g}$ of radium are available from the National Bureau of Standards. The procedure consists in fusing the rock in a vacuum furnace and flushing the radon with purified nitrogen into an ionization chamber where the separate ion pulses due to the alpha particles of the disintegrating radon and RaA are detected and amplified for counting. The chamber can be calibrated by introducing known amounts of radon into the chamber and determining the counting rate. When carefully purified nitrogen is used, the pulses in a cylindrical ion chamber of about 2 liters volume with a central electrode are sufficiently sharp for amplification by a conventional resistance capacity coupled amplifier.

For rocks in which some question regarding loss of radon through the surfaces exists, it is preferable to dissolve the rock and store the solution in a flask, with connections for removing the radon. The amount of radium in the solution can then be determined from the amount of radon collected over a definite period of time.

A diagram of the equipment for measurement of radium in rocks is shown in figure 87. $I$ is the ion-counting chamber which may be evacuated by connection to a pump at (5). $S$ is a flask containing the standard radium solution. To calibrate the chamber nitrogen is introduced at (1) and bubbled through the heated solution, passing through a furnace containing reduced copper, C, and drying bulbs, $B_{1}, B_{2}$ on the way to the evacuated chamber. A flask similar to $S$ is connected at (3) containing the solution of rock to be measured, from which radon may be transferred to the calibrated chamber for measurement. To rejuvenate the copper, hydrogen may be introduced at (4) and the water vapor permitted to escape at (2).

Thorium may be measured in a similar manner. However, the short half-value period of thoron requires that the gas containing the thoron be circulated at a constant rate through the thorium solution and the chamber. Standards consist of thorium that has been separated chemically from the ore for a period of at least 40 yrs. so that the decay products have come practically into equilibrium. Such standards will contain ionium, but the amount of radium will be negligible. In any case, the gas containing the thoron must be circulated at such a rate, to obtain adequate amounts of thoron for measurement, so that any radon present contributes a negligible number of alpha particles.

\section{Pleochroic Halves}

Radiation produces discoloration of many transparent materials. Mica is a substance that readily responds to the intense ionization produced by alpha rays and exhibits degrees of coloration depending on the intensity of the bombardment. Various types of mica, such as biotite, cordierite, and muscovite which have small circular discolorations that have a pattern of rings of varying graduations are found in nature. When examined with polarized light, they reveal the colors characteristic of pleochroism from which they derive their name. Joly [93] was first to show that these haloes were produced by small deposits of radioactive elements. Actually the haloes are spherical in homogeneous material and composed of concentric shells of various degrees of coloration. In a plane section the rings correspond to the ranges of the alpha particles that were emitted by the decay products of the radioactive element originally deposited.

These haloes have not provided very accurate measurements of geologic time, since some of the quantities entering into such a computation, such as the quantity of the radioactive element originally deposited, cannot be determined except by rough estimates. However, they do provide information regarding the ranges of alpha particles and decay periods of well-known radioactive elements over a period of the order of millions of years, revealing that no changes have occurred in this long interval of time.

\section{Generation of Heat in the Earth}

The accumulation of data that reveals that radioactive substances are widely distributed in the earth's crust has provided partial solution to the problem of the cooling of the earth. Previous to this knowledge it had been assumed that the earth, subsequent to its separation from the sun, had proceeded to cool until it reached its present state. However, the best available estimate would require about 20 million years for this process, whereas by radioactive methods, geological formations having ages several times this value have been studied. An estimate of the amount of heat developed by the radioactive substances in the earth provides an explanation for the regarded rate of cooling.

The principal substances providing heat of radioactive origin in the earth are uranium, thorium, and potassium. These substances, with their decay products, provide roughly $0.8,0.2$, and 0.00001 calories per gram per year. The low value for potassium does not mean that it is an unimportant source of heat. In fact, the relative abundance of potassium results in a contribution of the order of one-tenth of that of uranium and thorium. For a quantitative esti- 
mate of the heat developed in the earth from these substances, information is required regarding their total amounts in the earth. No definite information is available on this subject. However, if the surface distribution of radioactivity is maintained to a depth of only $16 \mathrm{~km}$ it has been estimated that this would be sufficient to balance the loss of heat by the earth. On the other hand, if the surface distribution is typical of that throughout the earth then the amount of heat generated is of the order of 200 times that lost to outer space. These estimates reveal the importance of the widespread distribution of radioactivity in maintaining the temperature of the earth for an almost unlimited duration of time.

\section{HEALTH PROTECTION}

A few general remarks on handling radioactive substances from the point of view of personal injury are included in this section. They are intended as a rough outline of the nature of the problems encountered with suggestions for the provision of reasonably safe conditions. It must be noted, however, that there is considerable divergence of opinions regarding tolerance levels of radiation. As long as these factors are based on opinions, they will be the basis for arguments. When better biological data are available, it is to be anticipated that the question of tolerances will be answered authoritatively. In the meantime investigators and technicians should exercise all reasonable care to maintain exposures well below currently acceptable levels in all cases where this can be done.

\section{Nature of Radiation Injuries}

It is well known that radiation absorbed in the body produces undesirable effects whenever the absorbed energy exceeds certain amounts to which the body has become accustomed, in its natural surroundings. Investigation has shown that injuries to living cells are in general proportional to the ionization produced in their structure. However, injuries vary for different types of ionization, the intense local ionization produced by alpha particles and protons being more damaging than the less densely localized ions produced by gamma rays, for example.

It is the lethal effect of ionization on individual cells that plays the dominant role in the radiation injuries in the body. However, an additional factor is present when comparing the effect of radiation on individual cells and that produced on an animal as a whole. This is the ability of many types of cells to replace destroyed cells. Therefore, radiation sufficiently intense to destroy only a small fraction of the cells produces no permanent injury, provided sufficient time elapses between exposures to permit recovery from the damage. This is the basis of the commonly accepted ideas regarding tolerance limits of exposure to radiation. However, not all types of cells can cooperate to re- pair damage of this type. For example, parts of the eye, brain, and some muscular tissue, cannot produce new tissue to replace that which has been destroyed.

\section{Genetic Effects of Radiation}

In recent years investigations have revealed that cells of the body can suffer damaging alteration without being destroyed by radiation. This is accomplished by altering the structure of the chromosomes, the thread-like structures in the nuclei of the cells that control the characteristics of the organism. If this occurs in a cell that later divides, the alteration affects the characteristics of the descendants of the altered cell. In this process the intricate system of elements in the chromosomes, called genes, which are assumed to have a definite number and arrangement for each type of cell, play an important role. The effect of the radiation may be to destroy or alter the chromosomes or genes, or both. The result is a mutation in which the subsequent cells differ from the parent cell. This genetic effect of radiation has two important implications for man. In one case cells iltered in this way may produce abnormal orrans in the body. In the other, if the change nas been produced in a germ cell, subsequent offspring may develop abnormally. The importince of these possibilities becomes more promnent in the light of knowledge that mutation shanges are irreversible. There is no recovery, and if a certain dose of radiation will produce a certain percentage increase in mutations it does not matter whether this dose is administered over a few days or a few years. It also appears to be well established that the percentage change in mutations is directly proportional to the dose. The combination of this fact and the condition of irreversibility indicates that genetic effects of radiation are permanently cumulative.

These facts have an important bearing on tolerance limits of exposures of individuals to radiation, particularly to penetrating radiations where the germ cells may be affected. It is obvious that so far as man is concerned, no information is available nor will become available 
within present generations, to determine what intensities of radiation can be tolerated over a life span without producing a noticeable change in the mutation rate. This problem is further complicated by the fact that most mutations are recessive and result in deleterious changes. This means that effects will not ordinarily appear in the first succeeding generation. Whereas a low mutation rate is normally present, any marked increase could not be tolerated. Although much more information is required on this subject before definite conclusions can be drawn, it appears unlikely that exposures currently recommended will produce significant genetic effects. This view is further strengthened when it is remembered that a small percentage of the population is exposed to these levels of radiation.

\section{Visible Evidence of Injury from Radiation}

The disruptive effects that radiation can produce in man may result in a great variety of bodily injuries in persons exposed to them. These effects depend on the individual, the type of radiation, particularly its penetrating power, and on the method of exposure, whether partial or over the whole body. The injuries range all the way from slight erythema to radiation osteitis. In many cases anemias occur that may be aplastic or regenerative, depending on the cell groups affected. Leucopenias are commonly associated with intense doses over a short period of exposure to hard X-rays or gamma-rays. Any of these effects may prove fatal if exposures are prolonged or repeated at too-frequent intervals. It is fortunate, however, that these and other symptoms and disabilities attendant on exposures to radiation are increasingly infrequent, now that sufficient information is available regarding presently known radiations to provide protective measures to prevent all but the fool-hardy or grossly ignorant from suffering any of the more serious consequences of over-exposure. However, a word of warning is not out of place in regard to the production of radiations of energies well beyond those that have been investigated. It is not improbable that new effects either in kind or degree will be discovered, and considerable caution is required until the biological effects of superenergy radiations have been investigated.

\section{Tolerance Limits}

The limits of exposure can be expressed in terms of units based on the energy absorbed in the body, that is, the roentgen for X- and gamma rays, the roentgen-equivalent-biological for beta rays, protons, slow neutrons, and alpha particles and roentgen-equivalent-man for fast neutrons. The commonly accepted tolerance level for $\mathrm{X}$ - and gamma-rays has been $0.1 \mathrm{r}$ per 8-hr. day, or 12.5 milliroentgens per hour. Evidence is accumulating that this level is probably too high, even apart from genetic effects. It can be expected that it will be revised downward. Tolerance levels in terms of rep for other types of radiation are determined from the relative biological effectiveness of each particular type.

\section{Measurement of Tolerance Levels}

Since all tolerances are referred basically to the roentgen, instruments for determining tolerance levels must be designed to measure the effect of radiation and calibrated in terms of this unit. This is particularly important when several types of radiation are present simultaneously. This limits survey meters to ionization chambers of special design.

At present the number of satisfactory instruments commercially available is limited. Increasing demands can be expected to lead to increase in the number and improvement of the quality. For gamma-ray exposures, including the effects of slow neutrons as far as they produce induced activities emitting gamma rays, pocket chambers of the type originally developed by Victoreen provide satisfactory measurement of the daily dose. This assumes that the chambers are provided with adequate insulation and protected from unnecessarily rough handling. For integrating exposures over longer periods, photographic films worn on the person are useful. Careful calibration and photometric measurement of the developed films are required to make this method reliable. Furthermore, the variation of the sensitivity of photographic emulsions with the quality of the radiation may lead to serious errors of interpretation.

\section{Alpha Radiation}

The most serious hazard in connection with substances emitting alpha radiation arises when these substances are taken into the body. The radium poisoning of radium dial painters in the approximate interval 1918-25 brought this aspect of radiation injury to light. When substances emitting alpha rays are ingested or are otherwise assimilated into the body and are then localized by biological actions into particular tissues, these tissues are exposed at close range to the powerful bombardment of this densely ionizing radiation. In the case of radium, there was preferential localization in the bone marrow where the red cells of the blood are manufactured. In those cases where relatively large amounts of radium were deposited, from 2 to $100 \mu \mathrm{g}$, in the skeletal tissue, fatalities resulted. Damage was 
greatly increased by the slow rate of elimination of ingested radium from the body.

Radium in the body can be detected and estimated qualitatively by measuring the radon in the exhaled breath. It can also be detected by Geiger-Müller counters, though less reliably, since contamination external to the body may contribute to the readings. However, radioisotopes that do not produce radon or similar emanation and that have no strong gamma rays, such as polonium and plutonium, cannot be readily detected in the living body. This circumstance calls for even greater precautions in handling.

\section{Protective Precautions for Substances Emitting Alpha Rays}

The nature of the problem encountered with substances emitting alpha rays suggests quite obviously the procedure for reducing hazards. Absolute radioactive cleanliness must be achieved, so that only minimal amounts may be inhaled or ingested. This cleanliness must be enforced by careful survey, by use of instruments specifically sensitive to alpha rays to determine that work tables, apparatus, the hands of the workers, and the workroom air are free from injurious amounts of contami- nation. Various arbitrary limits have been suggested for various elements, depending on their tendency to localize in the body and their rate of elimination. It is sufficient to note here that these limits should always be set as low as is practicable.

\section{Protection from Gamma Rays}

In maintaining gamma-ray exposures below any prescribed measurable tolerance level, three general methods of reducing exposures are available: (1) maintain a sufficient distance between the source and operator, thus taking advantage of the inverse square law; (2) interpose relatively thick shields of matter between source and operator; (3) perform all operations where the operator is required to be in the vicinity of the source as rapidly as possible. Under certain circumstances any one of these measures may be adequate, whereas under other conditions they must be used together to achieve the required protection.

Where relatively large numbers of workers are engaged in operations with radioactive material, a general program of inspection, measurement, and enforcement of regulations is required to provide adequately safe average conditions. 
[1] E. Rutherford, Phil. Trans. Roy. Soc [A] 204, 169 (1904).

[2] C. D. Ellis and W. A. Wooster, Proc. Roy. Soc. [A] 114, 266 (1927).

[3] P. J. VanHeerden, the crystal counter. (N. V. Noord-Hollandsche Uitgevers Maatschappij, 1945).

[4] R. C. Waddell, Rev. Sci. Instr. 10, 311 (1939).

[5] A. Trost, Z. Physik 105, 399 (1937).

[6] L. I. Schiffand and R. D. Evans, Rev. Sci. Instr. 7, 456 (1946).

[7] J. R. Pierce, Bell Lab. 16, 305 (1928).

[8] W. Orthmann, Z. Physik 60, 137 (1930).

[9] C. C. Lauritsen, Rev. Sci. Instr. 8, 438 (1937).

[10] J. S. Townsend, Phil. Mag. 6, 598 (1903).

[11] L. A. DuBridge and H. Brown, Rev. Sci. Instr. 4, 532 (1933).

[12] H. L. Anderson, A. W. Lawson, and G. L. Weil, Rev. Sci. Instr. 12, 511 (1941).

[13] J. M. Lafferty and K. N. Kingdon, J. Applied Phys. 17, 894 (1946).

[14] A. W. Hull, Physics 2, 409 (1932).

[15] H. LeCain and J. H. Waghoren, Can. J. Research 19, 21, 1941) ; H. Palevsky, R. K. Swant, and R. Grenchik, Rev. Sci. Instr. 18, 298 (1947).

[16] J. D. Cockcroft and E. Walton, Proc. Roy. Soc. 129, 477 (1930).

[17] J. Chadwick and M. Goldhaber, Proc. Roy. Soc. 151, 473 (1935).

[18] R. G. Herb, C. M. Turner, C. M. Hudson, and R. E. Warren, Phys. Rev. 58, 519 (1940).

[19] E. O. Lawrence and S. Livingston, Phys. Rev. 37, 1707 (1931).

[20] D. W. Kerst, Phys. Rev. 58, 841 (1940).

[21] E. M. MacMillan, Phys. Rev. 68, 143 (1945).

[22] W. Bothe and H. Becker, Z. Physik 66, 289 (1930).

[23] J. Chadwick, Proc. Roy. Soc. 136, 692 (1932).

[24] J. Chadwick and M. Goldhaber, Nature 134, 237 (1937).

[25] G. Breit and E. P. Wigner, Phys. Rev. 49, 519 (1936).

[26] H. Urey, F. Brickwedde, and G. Murphy, Phys. Rev. 37, 1669 (1931).

[27] W. Bleakney, Phys. Rev. 39, 164 (1932).

[28] J. R. Oppenheimer and M. Phillips, Phys. Rev. 48, 500 (1935).

[29] J. M. Cork and J. Halpern, Phys. Rev. 57, 667 (1940).

[30] E. Rutherford and T. Royds, Phil. Mag. 17, 281 (1909).

[31] G. H. Briggs, Proc. Roy. Soc. 118, 549 (1928).

[32] R. H. Birge, Rev. Mod. Phys. 13, 233 (1941).

[33] M. G. Holloway and M. S. Livingston, Phys. Rev. 54, 18 (1938).

[34] W. B. Lewis and C. E. Wynn-Williams, Proc. Roy. Soc. 136,349 (1932).

[35] C. G. Darwin, Phil. Mag. 23, 901 (1912).

[36] A. King and W. M. Rayton, Phys. Rev. 51, 826 (1937).

[37] W. H. Bragg and R. Kleeman, Phil. Mag. 10, 318 (1905).

[38] H. Geiger and J. M. Nuttall, Phil. Mag. 22, 613 (1911).

[39] E. Rutherford, Phil. Mag. 11, 166 (1906).

[40] H. Geiger and E. Marsden, Proc. Roy. Soc. 82, 495 (1909).

[41] E. Rutherford, Phil. Mag. 21, 669 (1911).

[42] H. Geiger, Proc. Manchester Lit. Phil. Soc. 55, 20 (1911).

[43] H. Geiger and E. Marsden, Phil. Mag. 25, 604 (1913).

[44] G. Gamow, Z. Physik 51, 204 (1928).
[45] R. W. Gurney and E. U. Condon, Phys. Rev. 33, 127 (1929).

[46] J. J. Thompson, Phil. Mag. 44, 293 (1897).

[47] A. H. Bucherer, Z. Physik 9, 755 (1908).

[48] O. Klemperer, Phil. Mag. 20, 549 (1935).

[49] M. Deutsch, L. G. Elliot and R. D. Evans, Rev. Sci. Instr. 15, 178 (1944).

[50] A. Bouwers, Physica, 4, 200 (1937).

[51] C. D. Ellis and W. A. Wooster, Proc. Roy. Soc. 117, 109 (1927).

[52] B. W. Sargent, Proc. Roy Soc. 139, 659 (1933).

[53] E. Fermi, Z. Physik 88, 161 (1934).

[54] W. Heisenberg, Z. Physik 77, 1 (1932).

[55] P. Jordan and O. Klein, Z. Physik 45, 751 (1927).

[56] F. Kurie, J. Richardson and H. Paxton, Phys. Rev. 49, 368 (1936).

[57] N. Feather, Proc. Cambridge Phil. Soc. 34, 599 (1938).

[58] W. F. Libby and D. D. Lee, Phys. Rev. 55, 245 (1939).

[59] C. D. Anderson, Phys. Rev. 41, 405 (1932).

[60] C. D. Anderson and S. Neddermeyer, Phys. Rev. 50, 263 (1936).

[61] J. Street and E. Stevenson, Phys. Rev. 52, 1103 (1937).

[62] L. LePrince-Ringuet, S. Goredeztky, E. Mageotte and R. Richard-Foy, Phys. Rev. 59, 460 (1941).

[63] F. Basetti, Phys. Rev. 59, 706 (1941).

[64] J. J. Lord and Marcel Schein, Science 109, 114 (1949); E. W. Cowan, Science 108, 534 (1945).

[65] E. Gardner and C. M. G. Lattes, Science 107, 270 (1948).

[66] A. H. Compton, Phys. Rev. 21, 482 (1921).

[67] K. Siegbahn, Phys. Rev. 70, 127 (1946).

[68] W. Bothe and H. J. V. Baeyer, Z. Physik 95, 418 (1935).

[69] B. Rossi, Nature 125, 636 (1930).

[70] W. B. Lewis, Electrical counting (Cambridge University Press, Cambridge, Eng., 1943).

[71] B. B. Bensen, Rev. Sci. Instr. 17, 533 (1946).

[72] E. Fermi, Nature 133, 898 (1934).

[73] O. Hahn, L. Meitner, and F. Strassmann, Z. Physik 106, 249 (1937).

[74] O. Hahn and F. Strassmann, Naturwissenschaften 27, 11, 89 (1939).

[75] N. O. Lassen. Phys. Rev. 68, 142 (1945).

[76] N. O. Lassen, Phys. Rev. 69, 137 (1946).

[77] N. O. Lassen, Phys. Rev. 70, 578 (1946).

[78] A. G. Ward, Proc. Roy. Soc. 181, 183 (1942).

[79] A. Graves and R. Walker, Phys. Rev. 71, 1 (1947).

[80] H. Fesefeldt, Z. Physik 86, 605 (1933).

[81] J. Riedhammer, Naturwissenschaften 32, 70 (1944).

[82] R. Peierls, Proc. Roy. Soc. 149, 467 (1935).

[83] G. v. Hevesy and F. Paneth, Z. Anorg. Chem. 82, 323 (1913).

[84] G. v. Hevesy, Z. Physik 16, 52 (1915).

[85] B. W. Sakmann, J. T. Burwell, and J. W. Irvine, Jr., J. Applied Phys. 15, 459 (1944).

[86] E. J. Murphy, et al., J. Franklin Inst. 231, 357 (1941).

[87] R. D. O'Neal and G. Scharff-Goldhaber, Phys. Rev. 69, 368 (1946); G. R. Gamertsfelder and M. Goldhaber, Phys. Rev. 69, 369 (1946).

[88] Rev. Mod. Phys. 3, 427 (1931).

[89] E. U. Condon and L. F. Curtiss, Science 103, 712 (1946).

[90] Hevesy and Paneth, Radioactivity.

[91] F. F. Koczy, Nature 151, 24 (1943).

[92] S. Meyer, Naturwissenschaften 25, 764 (1937).

[93] J. Joly, Phil. Mag. 13, 381 (1907).

WASHINGTON, FEBRUARY 1, 1949.

U. S. GOVERNMENT PRINTING OFFICE: 1949-831765 

